\title{
annual report 1976
}

nuclear physics laboratory

".niversity of washington

Whith DOE/ER-01388-345 


\section{DISCLAIMER}

This report was prepared as an account of work sponsored by an agency of the United States Government. Neither the United States Government nor any agency Thereof, nor any of their employees, makes any warranty, express or implied, or assumes any legal liability or responsibility for the accuracy, completeness, or usefulness of any information, apparatus, product, or process disclosed, or represents that its use would not infringe privately owned rights. Reference herein to any specific commercial product, process, or service by trade name, trademark, manufacturer, or otherwise does not necessarily constitute or imply its endorsement, recommendation, or favoring by the United States Government or any agency thereof. The views and opinions of authors expressed herein do not necessarily state or reflect those of the United States Government or any agency thereof. 


\section{DISCLAIMER}

Portions of this document may be illegible in electronic image products. Images are produced from the best available original document. 
(2)

The United Stas Covent. Neither the

Uneed Stas nor the United States Department of

contractors, subcontractors, or their employees, mekes

any warranty, express or implied, or assumes any legal

liability or responsibjity for the accuracy, completeress

or usefulness of any information, apparatus, product or

process disclosed, or represents that its use would not infringe privately owned rights.

\footnotetext{
ANNUAL REPORT

Nuclear Physics Laboratory University of Washington

June, 1976
} 
For our cover picture we continue the tradition of the past several years and show the photograph of the high pressure gas cylinders which store the nitrogen and carbon dioxide mixture used for insulating the high potential terminals of the two Van de Graaff machines. They form a group of towers which front on the Van de Graaff building and have aroused considerable local interest and curiosity. 


\section{INTRODUCTION}

This Annual Report describes activities at our Laboratory for the period from Spring 1975 to Spring 1976.

Some new sections have been added this year and others have been omitted or significantly reduced, reflecting changes in our interests and those of the nuclear physics community as a whole. It perhaps would be fitting to note particularly that for the first time in the more than 20 years during which these Reports have been issued, there is no section on fission research. Similarly, far less work on light ion scattering is reported here than has appeared in past Reports.

The most marked increase in Laboratory activity in the past few years has been in heavy ion physics. In consequence, three Chapters of the 1976 Report are devoted to this general area. There also is increased focus on problems of fundamental symmetries in nuclei, and this is reflected in a new Chapter heading. Other active research areas are continuing at levels similar to those of preceding years. One of our newer programs, the users' activity at LAMPF, is just completing two experiments on pion cross section measurements and is scheduled for another experiment at LAMPF this autumn.

Our interest remains high in a room temperature linac postaccelerator to be injected by the Van de Graaff accelerators. Design plans for a postaccelerator dominate the accelerator development chapter.

We close this introduction with the standard reminder that the articles in this report describe work in progress and are not to be regarded as publications nor quoted without permission of the investigators. The names of the investigators on each article have been listed alphabetically but where appropriate the name of the person primarily responsible for the report has been underlined.

As always, we welcome applications from outsiders for the use of our facilities. As handy reference for potential users we list in the table on the following page the vital statistics of our accelerators. For further information please write or telephone Dr. W.G. Weitkamp, Technical Director, Nuclear Physics Laboratory, University of Washington, Seattle, WA 98195; (206) 543-4080. 
THREE STAGE TANDEM VAN DE GRAAFF ACCELERATOR

(A High Voltage Engineering Corp. Model FN)

Completed: 1967

Funding: Purchased with NSF funds; maintained by ERDA funds and some funds from the State of Washington.

Beams currently available: (See also W.G. Weitkamp and F.H. Schmidt "The University of Washington Three Stage Van de Graaff Accelerator" Nucl. Instrum. Methods 122, 65 (1974).

Ion

$$
\begin{array}{cccc}
\text { Typ.Current " Max.Practical } & \text { Typ.Current } & \text { Max.Practical } \\
2 \text { stage }(\mu \mathrm{A}) & \text { Energy } 2 \text { Stage } & 3 \text { stage( }(\mu \mathrm{A}) & \text { Energy } 3 \text { Stage } \\
& (\mathrm{MeV}) & (\mathrm{MeV})
\end{array}
$$

$\begin{array}{lcccc}\text { P,d } & 15 & 18 & 10 & 25 \\ \text { polarized p, } & 0.1 & 18 & -- & -- \\ 3,4 \mathrm{He} & 3 & 27 & -- & -- \\ 6,7 \mathrm{Li} & 0.5 & 36 & -- & 70 \\ \mathrm{C} & 2 & 63 & 1 & 79 \\ \mathrm{~N} & 2 & 72 & 1 & 88 \\ 0 & 10 & 81 & 3 & -- \\ \mathrm{Si} & 0.2 & 30 & -- & 124 \\ \mathrm{Cl} & 0.2 & 117 & 0.2 & \\ \mathrm{Ni} & 0.5 & 117 & & \\ \mathrm{Br} & 0.1 & 125 & & \\ \mathrm{Ag} & 0.01 & 125 & & \end{array}$

CYCLOTRON

(A Go-inch fixed energy machine)

Completed: 1952

Funding: Constructed primarily with state fundo and sulsequentiy supported by AEC funds. Now sustained by funds from outside users.

Reams currentily avaliable:

Tul Typal Current $(\mu \mathrm{A}) \quad$ Maximum Practical
Fnergy $(M \in V)$

$\begin{array}{ccc}p & 100 & 11 \\ d & 150 & 22 \\ { }^{4} \mathrm{He} & 30 & 42\end{array}$


TABLE OF, CONTENTS

1. ACCELERATOR DEVELOPMENT

1.1 Van de Graaff Accelerator Operations and Improvements $\quad 1$

1.2 Cyclotron Operations and Improvements . . 4

1.3 Design and Construction of Electronic Equipment 5

1.4 Measurements of a Model Spiral Resonator Cavity 7

1.5 An Improved Design for a Spiral-Resonator Postaccelerator
for the UW Three Stage FN Tandem Accelerator

1.6 Design of a Prototype Spiral Resonator Cavity 13

1.7 A $108.6 \mathrm{MHz}$ Buncher for 2- and 3-Stage Tandem Operation 14

$\begin{array}{lll}1.8 & \text { Production of Very Thin Self-Supporting Oil Films for } \\ \text { Stripping and Target Applications } & 18\end{array}$

1.9 OSHA-WISHA Compliance $\quad 20$

2. ION SOURCE DEVELOPMENT

2.1 Polarized Ion Source Development 22

2.2 Sputter Ion Source 26

3. INSTRIMENTATION, DETECTORS, RESEARCH TECHNIQUES

3.1 Silicon Detectors . 28

3.2 Further Development of a Gas Cell-Detector System 28

3.3 Gas Ionization Cuinter Telesrnpe 29

3.4 A Permanent Electronics Selup for High Resolution Timing . 31

3.5 Development of Electron Detector Telescopes 33

3.6 Construction of Scdltering Chambers for Particle-Gamma
and Gamma-Gamma Coincidence Experiments

3.7 Feedback of Spin Direction of the Polarized Beam 36

4. THE COMPUTER AND COMPUTING

4.1 Computer System Improvements. 38 
4.2 Revision of the CALCOMP Plotting System 38

4.3 Graphics on the Computer 2 Display 39

4.4 HOP-TWO: Version 4.1 for Iterated Optical Model

4.5 COULIT, A Compact Coulomb-Nuclear Inelastic Scattering
DWBA Program

4.6 GELIFIT: A Peak and Background Fitting Program for Ge(Li)
$\gamma$-Ray Energy Spectra

5. ATOMIC PHYSICS

5.1 The Question of Electron Pickup During the a Decay of $210 \mathrm{Po}$

5.2 Cross Sections for K-Shell Ionization by Electron Impact 45

5.3 Multiple Scattering of Heavy Ions 46

5.4 Search for the Double Photon Decay of $\mathrm{Pb}^{+}\left(1 \mathrm{~s}^{-1}\right) \quad 47$

5.5 K-Shell Ionization in $\alpha$ Decay vs $\alpha$ Collisions 48

5.6 Parameterization of Ionization Probabilities in Ion-
Atom Collisions

$5.7 \mathrm{~K}$-Shell Ionization of $\mathrm{Pb}$ by Heavy Ions 50

6. NUCLEAR ASTROPHYSICS

6.1 Gamma-ray Production Cross Sections 51

6.2 Deuterium Production and Li Destruction Cross
Sertions

7. FUNDAMENTAL 3YMMETRIES IN NUCLEI

7.1 Parity Mixing in ${ }^{18} F$

$\%$ A New Experiment to Measure the Parity Mixing in ${ }^{19} \mathrm{~F} \quad 58$

7.3 A Redetermination of the Effective ${ }^{19} \mathrm{~F} *$ Polarization in the $19 \mathrm{~F}$ Parity Mixing Experiment

8. NUCLEAR STRUCTURE

8.1 Gamma Decays of the Lowest $T=3 / 2$ Levels in ${ }^{9} B$ and ${ }^{9} B$ 
8.2 Isospin Mixing in ${ }^{12} \mathrm{C}$

8.3 Charged Particle and $\gamma$ Ray Decays of the Lowest $\mathrm{T}=2$ States of ${ }^{24} \mathrm{Mg},{ }^{28} \mathrm{Si}, 32 \mathrm{~S}$ and ${ }^{44} \mathrm{Ti}$

8.4 Charged Particle and Neutron Decays of the Lowest $\mathrm{T}=2$ States in ${ }^{8} \mathrm{Be},{ }^{12} \mathrm{C}$ and $16_{\mathrm{O}}$

8.5 Lifetimes of Isomeric States in ${ }^{174} \mathrm{HF}$

8.6 Study of Low Lying Dipole States in $\mathrm{Pb}$ Isotopes via Analog Resonant $\left(\mathrm{p}, \mathrm{p}^{\prime} \gamma\right)$

9. RADIATIVE CAPTURE MEASUREMENTS AND CALCULATIONS

9.1 The ${ }^{11}{ }_{B(p, \gamma)^{12}}$ Reaction

9.2 The ${ }^{14} \mathrm{C}(\overrightarrow{\mathrm{p}}, \gamma){ }^{15}$ Reaction

9.3 Direct-Semidirect Calculations in Light Nuclei 84

9.4 The ${ }^{89} \mathrm{Y}(\mathrm{p}, \gamma)^{90} \mathrm{Zr}$ Reaction $\quad 87$.

9.5 Direct-Semidirect Calculation in the $\mathrm{Pb}$ Region 88

9.6 Radiative Capture of Fast Neutrons 91

10. SCATTERING AND REACTIONS

10.1 Excitation Functions and Angular Distributions for Inelastic $4 \mathrm{He}$ scattering to the $\left(0^{+}, 6.44 \mathrm{MeV}\right)-$ State in $24 \mathrm{Mg}$

11. REACTION WITH POLARIZED PROTONS AND DEUTERONS

11.1 A l'ensor Deuleron Bcam Polarimeter Based on the ${ }^{3} \mathrm{He}(\mathrm{d}, \mathrm{p})^{4} \mathrm{He}$ Reaction

11.2 Elastic Scattering of Polarized Protons from Carbon

11.3 Analyzing Power in the ${ }^{207} \mathrm{~Pb}\left(\overrightarrow{\mathrm{p}}, \mathrm{P}_{0}\right){ }^{207} \mathrm{~Pb}$ Reaction Near the $3 p_{1 / 2}$ Isobaric Analog Resonance

11.4 Analyzing Powers for the Continuum Portions of Particle Emission Spectra

12. HEAVY ION ELASTIC AND INELASTIC SCATTERING

12.1 Evidence for Shallow Strongly Absorbing Heavy-Ion Optical Potentials 
12.2 Application of the Austern-Blair Theory to Nuclear-

Coulomb Interference in Heavy Ion Inelastic Scattering

12.3 Notch Perturbation Tests of the Sensitivity of the Heavy

Ion Optical Model to the Details of the Nuclear Potential

12.4 Elastic and Inelastic Scattering of ${ }^{6} \mathrm{Li}$ by ${ }^{40} \mathrm{Ca}$ and ${ }^{44} \mathrm{Ca} \cdot 118$

12.5 Elastic Scattering of ${ }^{14} \mathrm{~N}$ by ${ }^{12} \mathrm{C}$ from 33.0 to $48.0 \mathrm{MeV} \quad 123$

12.6 The Elastic Scattering of ${ }^{16} 0$ from ${ }^{14} \mathrm{C} \quad 125$

12.7 Elastic Scattering of ${ }^{28} \mathrm{Si}$ from ${ }^{26} \mathrm{Mg} \quad 127$

12.8 Effects of Non-loral Potentials in Heavy lon Reactions 128

13. HEAVY ION DEEPLY INELASTIC AND FUSION REACTIONS.

13.1 X-ray Technique for Measurement of Heavy Ion Nuclear. Charge Distributions

13.2 A New Technique for Nuclear Lifetime Measurement in the Attosecond Range

13.3 Study of Excitation Functions for ${ }^{16} 0$ and ${ }^{18} 0$ Induced Reactions on ${ }^{12} \mathrm{C}$ Using $\gamma$-Ray Spectroscopy

13.4 Deeply Inelastic Scattering of ${ }^{35} \mathrm{Cl}$ by ${ }^{59} \mathrm{Co}$

13.5 Kr and Xe Induced Reactions 144

14. HEAVY ION TRANSFER AND TNTFRMEDIATE ETRUCTURE REAC'I'IONS

14.1 Studies of the Reactions ${ }^{12} \mathrm{C}\left({ }^{14} \mathrm{~N},{ }^{13} \mathrm{~N}\right){ }^{13} \mathrm{C}$ and ${ }^{12} \mathrm{C}\left({ }^{14} \mathrm{~N}, 13_{\mathrm{C}}\right) 13_{\mathrm{N}}$ at $53.5 \mathrm{MeV}$

14.2 l'he ${ }^{12} \mathrm{C}+{ }^{16} \mathrm{O}+{ }^{8} \mathrm{Be}+{ }^{20}$ Ne Reaction 152

14.3 The ${ }^{12} \mathrm{C}\left({ }^{18} \mathrm{O}, \alpha\right)^{26} \mathrm{Mg}$ Reaction 158

15. MEDIUM ENERGY PHYSICS

15.1 Total Pion Cross-Sections 158

15.2 Ratios of ${ }^{1 I_{C}}$ Activations Induced by Pions on Various
Targets

15.3 Excitation of Giant Resonances by Inelastic Pion
Scattering 
16. ENERGY STUDIES

16.1 Energy Studies

17. RESEARCH BY USERS AND VISITORS

17.1 Fast Neutron Beam Radiotherapy -- Medical Radiation Physics 164

17.2 Fast Neutron Beam Radiotherapy -- Experimental Oncology 165

17.3 Fast Neutron Beam Radiotherapy -- Radiobiology · 167

17.4 Fast Neutron Beam Radiotherapy -- Clinical Program 169

17.5 Total Body Calcium by Neutron Activation Analysis Techniques: Applicability to Bone Wasting Disease, and Space Flight Related Bone Loss

17.6 Nuclear Pharmacy 170

17.7 Alpha Particle Injection into Reactor Materials . 171

17.8 Delayed Neutron Spectra Measurements . I71

17.9 Optical Properties of the Alkali Halides . . 173

17.10 Hyperfine Structure Anomalies in the Cadmium Isotopes $\quad 174$

17. Il The Role of Compound Nuclear Angular Momentum in Determining Fission Fragment Angular Momentum $\quad 174$

17.12 Gamma-Ray Angular Correlations in Odd-Mass Nuclei $1 \% 5$

17.13 The Helium Ion-Induced Fission of Enriched Isotopes of
Mercury and Tungsten at Moderate Bombarding Energies

17.14 The ${ }^{89} \mathrm{Y}(\mathrm{p}, \mathrm{Y})^{90} \mathrm{Zn}$ Reaction below the Giant Dipole Reșonance . 176

17.15 Radiative Proton Capture by Carbon-12 178

17.16 Plastic Tráck Detector Calibration 180

18. APPENDIX

18.1 Nuclear Physics Laboratory Pensonnel 181

18.2 Advanced Degrees Granted, Academic Year 1975-1976 183

18.3 List of Publications $\quad 184$ 


\section{ACCELERATOR DEVELOPMENT}

\subsection{Van de Graaff Accelerator Operations and Improvements}

W.G. Weitkamp and Staff

The operation of the two Van de Graaff voltage generators comprising the three-stage accelerator was a study in contrasts during the past year. The positive terminal tandem ran reliably and stably, while the negative terminal injector provided us with our most serious maintenance problem. Statistics for the operation of both machines are given in Table 1.1-l.

Tandem operations and improvements. The tandem operated for a significant fraction of the year at or near its maximum voltage of $9 \mathrm{MV}$. There seldom was trouble in reaching this voltage despite the advancing age of the beam tubes, now 45,000 hours on sections 1, 2 and 3, and 61,000 hours on section 4; they show only minimal evidence of damage. The locally built column resistorsl appear to have stood up well, with no replacements required. The tank was opened 14 times, mostly to replace stripper foils, reflecting the short foil lifetime for heavy ions, the high usage of heavy ion beams, and the small capacity of the foil holder ( $40^{\circ}$ foils). The charging belt was replaced twice; tearing of the belt fabric during normal operation was the reason for replacement both times.

Several significant improvements have been made to the tandem. The mercury diffusion pump at the low-energy end has been replaced by an ion pump. This has reduced operating costs by saving liquid nitrogen and by reducing maintenance, and has simplified the vacuum system. Further replacements of this type are planned for the future. The direct extraction ion source has been improved by replacing the manual gas needle valve by a motor driven valve, by reinforcing the high voltage insulation, and by simplifying the vacuum system control panel. Other improvements to ion sources are described in Secs. 2.1 and 2.2.

A bromine beam was accelerated for the first time this year. A mixture or 2 per cent freon $13 \mathrm{BI}\left(\mathrm{CBrF}_{3}\right)$ in hydrogen gas was used in the direct extraction ion source. Approximately $1.3 \mu \mathrm{A}$ of negative beam was obtained.

Injector operations and improvements. In contrast to the tandem, the injector has had severe problems, causing it to be out of service for four monthe. In July 1975, the injector rather abruptly refused to operate above $4 \mathrm{MV}$; it had previously run satisfactorily up to $6.5 \mathrm{MV}$. An extensive series of diagnostic tests and maintenance procedures was carried out to try to determine the cause of this drop in maximum voltage. The properties of the charging system, the insulating gas, and the column structure were examined in detail. The charging belt was replaced and the column thoroughly cleaned and polished in regions of high electric field. Ultimately, the injector was run with both beam tubes and ion source removed. In all configurations we encountered tank sparking, nearly always downward from the ends of the terminal to the tank floor. The sparking.was random in time, except if the voltage was brought up too rapidly after a spark, in which case repeated sparking would occur.

No unambiguous cause of the sparking could be determined; the most reasonable hypothesis advanced was that some sort of dust appeared in the tank which 
would not precipitate readily from the insulating gas. Three possible sources of dust were belt fabric, desiccant dust from the driers, and bits of light pipe: left over from the failure of an attempt to use light pipes to read out terminal source parameters. In addition to the dust possibility, breakdowns in the beam tubes and faulty terminal voltage metering were apparently contributing causes of the injector difficulties. The injector has since been restored to full operation, and has run successfully at $7.8 \mathrm{MV}$.

In the course of repairing the injector, a number of new diagnostic techniques were developed and improvements made. We can, for example, now monitor the insulating gas humidity continuously with good precision. We have installed a remotely positionable sled on the column which permits us to short any desired $20 \mathrm{~cm}$ length of column to isolate regions of column breakdown. A revised charging current control automatically programs the charging current for optimum recovery from a terminal spark, preventing repeated sparking. Baffles and shielis have been installed in the terminal and along the column near the terminal to prevent dust produced and distributed by the charging belt from falling into the region of the accelerator with the highest electric field.

We have also used the shutdown of the injector necessitated by the voltage problem to make a number of other improvements: the terminal ion pump has been completely overhauled, and the terminal source metering system and the external beam handling system have been revised to improve reliability.

1. Nuclear Physics Laboratory Annual Report, University of Washington (1975), p. 5 . 
Table 1.1-1. Statistics of Van de Graaff Operation from April 16, 1975 to April 15, 1976

1. Division of time among activities

\begin{tabular}{cr} 
Time (Hrs) & Per Cent \\
6607 & 75 \\
1011 & 12 \\
260 & 3 \\
882 & 10 \\
\hline 8760 & 100
\end{tabular}

2. Division of beam time among particles

a. Two stage operation

Protons

900

14

Polarized protons

1344

22

Deuterons

152

Polarized deuterons

85

3

$3_{\mathrm{He}}$

${ }^{4} \mathrm{He}$

414

$\cdot 1$

${ }^{6} \mathrm{Li}$

341

3.09

7

$12 \mathrm{C}$

135

$14 \mathrm{~N}$

398

160

897

180

$28 \mathrm{Si}$

149

$35 \mathrm{Cl}$

32

134

${ }^{79} \mathrm{Br}$

Subtotal

24

5314

b. Three stage operation

Protons

$14_{\mathrm{N}}$

160

180

$35 \mathrm{Cl}$

Subtotal

959

TOTAL BEAM TIME

6273

3. Division of normal operation among activities

University of Washington Nuclear

Physics Laboratory

University of Washington Department

of Nuclear Engineering

University of British Columbia

Washington State University

Total $\frac{24}{6607}$

a) Includes all the time the accelerator was under the control of an experimenter.

b) This is the number of hours in a year. 


\subsection{Cyclotron Operations and Improvements}

\section{J.W. Orth and Staff}

This year the major effort of the cyclotron section went into complying with WISHA requirements and into improving patient handling facilities. Statistics of cyclotron operations are given in Table 1.2-1. It is interesting to note that the machine lost only 38 of the scheduled time this year because of unanticipated maintenance. This is the best record it has ever had.

Much thought went into designing a new protective interlock system that would provide both maximum safety and minimum interference with maintenance and operation. The system that evolved from discuseions with the campus Radiation Safety Uffice was to erect a fence and gate that separates the treatment area from the machine area. The gate was fitted with a key-operated electrical interlock, with appropriate contacte arranged to sound an alarm bell if hazardous conditions are present. The system also prevents the machine from being operated if any safety interlock is violated and unless all safety criteria are met. The system is also designed to prevent entry to the machine area even when the machine is off, thus protecting the unwary.

The treatment area has been improved with the installation of a new treatment chair. This flexible device allows the patient to be precisely positioned with a minimum of effort. The chair also allows the treatment of lower pelvic cancers which have hitherto been untreatable. The future expectations are for an increase in the scope of treatable cancers. The Cancer Therapy project is described in more detail in Secs. 17.1-17.4.

The weakest element in the cyclotron at present is the main magnet regulator. Steps are being taken to improve this: a new field regulator has been built and put into operation. This pointed out that a change in the main series transistor bank i.s nesessary. Thus tlie next step is to convert the germanium transistors to silicon. The design is completed and is awaiting shop time to complete construction. 
Table 1.2-1. Statistics of Cyclotron Operations from April 16, 1975 to April 15, 1976

1. Division of time activities

$\begin{array}{cr}\text { Time (hrs) } & \text { Per Cent } \\ 1489 & 92 \\ 74 & 5 \\ 52 & -\frac{3}{100} .\end{array}$

2. Division of beam time among projectiles

Alpha particles

Protons

Total

Deuterons

Total

\begin{tabular}{rr}
93 & 10 \\
4 & $<1$ \\
857 & 90 \\
\hline 954 & 100
\end{tabular}

3. Division of normal operating time among users

University of Washington Cancer Therapy Group
a. Therapy
633
43
b. Physics
320
21
c. Biology
151
10
d. Experimental Oncology
89
6
University of Washington Department of Nuclear Medicine
160
11
University of Washington Department of Nuclear Pharmacy
Atomics International
Oregon State University
University of Washington Physics Department
Seattle Veterans Administration Hospital

$\begin{array}{rr}12 & <1 \\ 75 & 5 \\ 7 & <1 \\ 28 & 2 \\ 10 & <1\end{array}$
University of Washingtin Department of Electrical
Engineering
Westerin Washington State College

\begin{tabular}{rr}
2 \\
2 \\
\hline 1489
\end{tabular}$\quad \begin{array}{r}<1 \\
<1\end{array}$

i.3 Design and Construction of Electronic Equipment

H. Fauska and R. Stowell

Electronic projects for general use:

(1) 2 dual switchable attenuators were constructed to facilitare experiment setup.

(2) 3 timing pre-amplifiers providing fast timing signal outputs wore constructed. 
(3) A 6-channel fast NIM logic level to slow NIM logic level converter was designed and constructed.

(4) An 8-channel add/subtract unit providing gating signals, a variable offset and delay was designed and constructed for use in particle identification setups.

(5) 4 channels of photomultiplier tube pre-amplifiers were constructed. The units are mounted in NIM standard plug-ins.

(6) 3 50-ohm switchable attenuators were constructed.

Electronics projects for the postaccelerator: (See Secs. 1.4 and 1.5 )

(1) A standing wave ratio unit was constructed to assist in cav1ry termination adjustments.

(2) A digital frequency stabilizer to provide selectible and stable signals from 80 to $130 \mathrm{MHz}$ for cavity testing was designed and constructed.

Electronics projects for the Van de Graff accelerator:

(1) An electrometer with logarithmic response over 5 decades to record ion source output currents was designed and constructed.

(2) A 3-channel ion gauge controller was designed and constructed for use on the polarized ion source (see Sec. 2.1).

(3) A dual channel dewpoint controller was constructed for the tandem and injector accelerators (see Sec. 1.1).

(4) A new tandem switching magnet degaussing power supply was designed and constructed to replace the original unit, whioh had becum unreliable.

(5) An analog differential sensor of tandem terminal potential was designed and constructed. The unit provides a gating signal to the experiment controller whenever the terminal voltage is within a specified range. This helps insure that data is taken only when the rmorr Ion opecies and charge state is present in the beam.

(6) A programmer for the tandem switching magnet to deflect the beam on and off a target and to provide appropriate gating signals to the experiment controller was designed and constructer.

(7) The belt charge control on the injector was replaced (see Sec. 1.1). The new unit still controls the belt charge from the generating voltmeter. The old tube pre-amp was replaced with a solid state unit. The new control system provides signals for hoth digital and the usual analog meter display of the terminal voltage. The original controller would allow sustained sparking to occur under certain conditions. To prevent such sparking a circuit was designed to sense the loss of generating voltmeter-signal during a spark, reduce the belt charge current for five seconds, and then return it to the same value as before 
sparking with a 35 second ramp. The new system performs very well.

Electronics projects for the cyclotron:

(1) A new main magnet generator field controller was designed and constructed to improve stability and reliability.

Electronics projects for medium energy research: (See Secs. 15.1-15.3)

(1) A multiple channel pulse differentiator was constructed.

(2) A pre-scaler chassis was constructed.

(3) An experiment duty cycle unit was designed and constructed. The unit provides a crystal controlled pulse train with three outputs to permit measurement of experiment dead time, live time or clock time. The sensor part has full updating capabilities. The time resolution is one microsecond.

(4) A target position programmer was designed and constructed. The programmer controls a hydraulic cylinder to position a target in each of two positions for 100 seconds with a 15 second transit time. The proper enable and routing gates are provided.

(5) A 75 ampere power supply to run the hydraulic pump and control was constructed.

1.4 Measurements of a Model Spiral Resonator Cavity

D.D. Chamberlin and J.G. Cramer

A model spiral resonator cavity with diameter and length both equal to $33 \mathrm{~cm}$ was constructed of copper. Flexible design allowed the insertion of various configurations of spirals and drift tubes, as well as other structures. Measurements were primarily concerned with the frequency spectra and shunt impedances of the resonant modes formed by varying the helicities, number, and spacing of the spirals and with the transit time factors for differing field profiles formed by varying the lengths and spacing of the drift tubes. Reliability of these measurements was enhanced by the construction and use of a digital frequency controller modifying the output of the basic oscillator, and was checked by using several glass balls and sapphire beads in the bead perturbation technique described in a previous report. 1

The resonant modes of the system can be labeled and identified by the symmetry of the voltages on the drift tubes and by the number of nodes in the overall field profile (see Ref. 1). Thus, a single spiral displays the Sl mode, while a three spiral system gives the modes S3, A2, and S1, in order of increasing resonant frequency. Attempts were made to develop a scheme whereby physical changes in the geometry would suffice to "tune" the system so that each of the three modes would occur at a common resonant frequency. These included reversing the helicity of one or more spirals, changing the length of one or more spirals, changing the effective length of the stem of the spirals, and changing the axial separation of the bases of the spirals. It now seems preferable to employ a 
separate and distinct geometry for each mode, with attendant simplifications in the mechanical design.

Measurements of shunt impedance were made for a broad range of configurations of number, spacing, and helicity of the spirals and of length, diameter, and spacing of the drift tubes. Lacking a general mathematical model which might predict shunt impedance for a given configuration, these empirical data form the basis for future choices of geometry. A set of spirals was constructed from flat strips of copper and shown to have larger shunt impedance due to the increased surface area and reduced resistance to the RF current flow in the spiral. This effect persisted for other spiral sets with the increased area of a. flat strip only on the outer turn or only on the stem of the spirals. A slight increase in shunt impedance was obtained by splitting the drift tubes, thus inhibiting circulating currents and lessening the axial magnetic fields. With the use of a movable end plate, the shunt impedance has been monitored as function of the overall cavity length, corresponding to a variety of optimum particle velocities. A crude set of chopper plates was installed on spirals and shown to have usable shunt impedance. A further set of five spirals also showed usable field profiles and shunt impedance.

Each of the shunt impedance measurements results in a field profile from which the transit time factor for particle accelcration can be calculated. These field profiles were varied by independent changes in the length of the drift tubes and in the size of the gaps between tubes. From these data, optimum transit time factors and field profiles can be obtained by separate designs of the drift tube and gap configurations for each resonant mode.

The measured shunt impedance of the optimum geometries is from 20 to 50 meg-ohm/meter with the relatively poor elentrical sontacto of this iludel cavity. Transit time factors exceeding 0.9 have been achieved. Although for these low power tests, it has not been necessary to adjust the input impedance of the cavity or to compensate for the flexing of the spirals and rosultant frequency shifts, both of these processes have been shown to be feasible.

1.5 An Improved Design for a Spiral-Resonator Postaccelerator for the UW Three Stage FN Tandem Accelerator

D.D. Chamberlin and J.G. Cramer

In last year's Annual. Report ${ }^{1}$ we described a feasibility study for a sprial resonator postaccelerator which would greatly enhance the beam energy performance of the Nuclear Physics Laboratory accelerator facility. Since that time, there have been two important developmente: (1) we have made considerable progress in the testing and understanding of these cavities (see Secs. 1.4 and 1.6 of this report) and (2) the Frankfurt group ${ }^{2}$ has been able to produce a mathematical model which permits the accurate prediction of shunt impedances and the optimization of cavity design. In the light of these developments it became desirable to reexamine the old design to see if it could be improved.

In particular, it was deemed highly desirable to reduce the power consumption of the design and "level" the power so that each cavity could be powered 
with the same $20 \mathrm{~kW}$ drive unit. Further, the old design, in its partial implementations had "crashes" in which above a particular beam mass the performance declined sharply. Also, the old design placed great emphas is on stripping the heavy ion beam three times, leading to considerable loss of intensity. Finally, as a result of considerable testing, the concept of using a single cavity excited in several resonant modes was dropped and a "convertible" cavity concept was substituted. This type of cavity (see Sec. 1.6) has an easily replaceable resonant electrode structure and thus can be quickly reconfigured to "shift gears" to higher or lower phase velocities for light or heavy ions.

The spiral-resonator linear accelerator design program SIMILAC $^{3}$ was extensively rewritten to include the new information based on the Frankfurt model. A technique was devised to avoid the "crashes" described above so that the performance falls off more gently with increasing beam mass, and full advantage was. taken of the convertible cavity concept in obtaining optimum performance. The optimization of the design was done assuming two rather than three strippings of the beam, so that optimum performance was assured in this mode of operation.
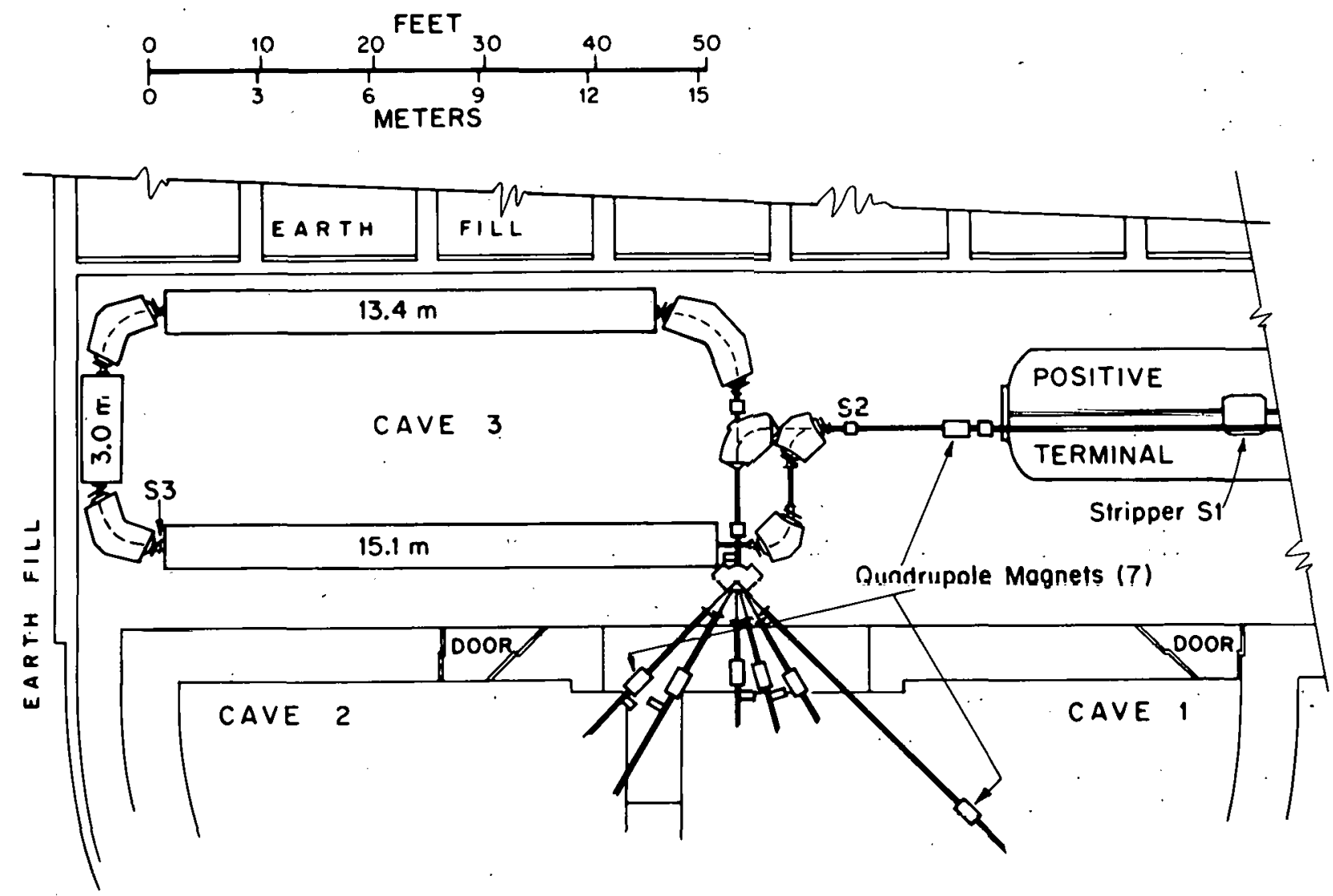

Fig. 1.5-1. Postaccelerator position in Cave 3 of existing tandem building. Stripper positions are indicated by labels S1-3, and resonator and quadrupole portions of linac are shown as rectangles with lengths given. Magnets are to scale for commercially available units. 


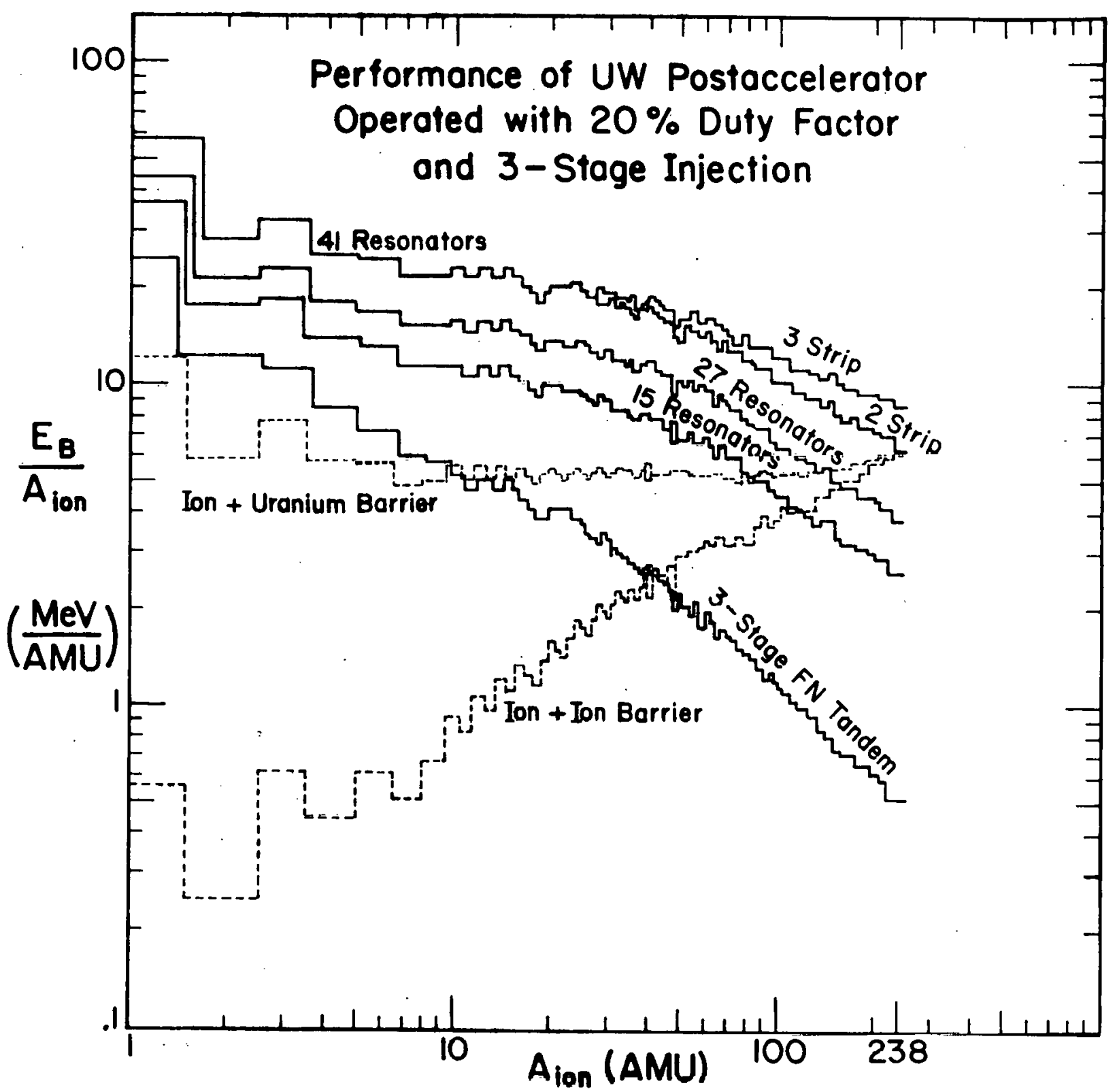

Fig. 1.5-2. Calculated energy performance of new postaccelerator design operated at $20 \%$ duty factor with beam injected from three stage FN tandem. Three configurations are considered with 15, 27, and 41 resonaton elementn, respertively, dul $\mathrm{RF}$ power consumption of $20 \mathrm{~kW} /$ resonator. The performance of the 41 resonator configuration is calculated with and without the use of the third stripper S3. 


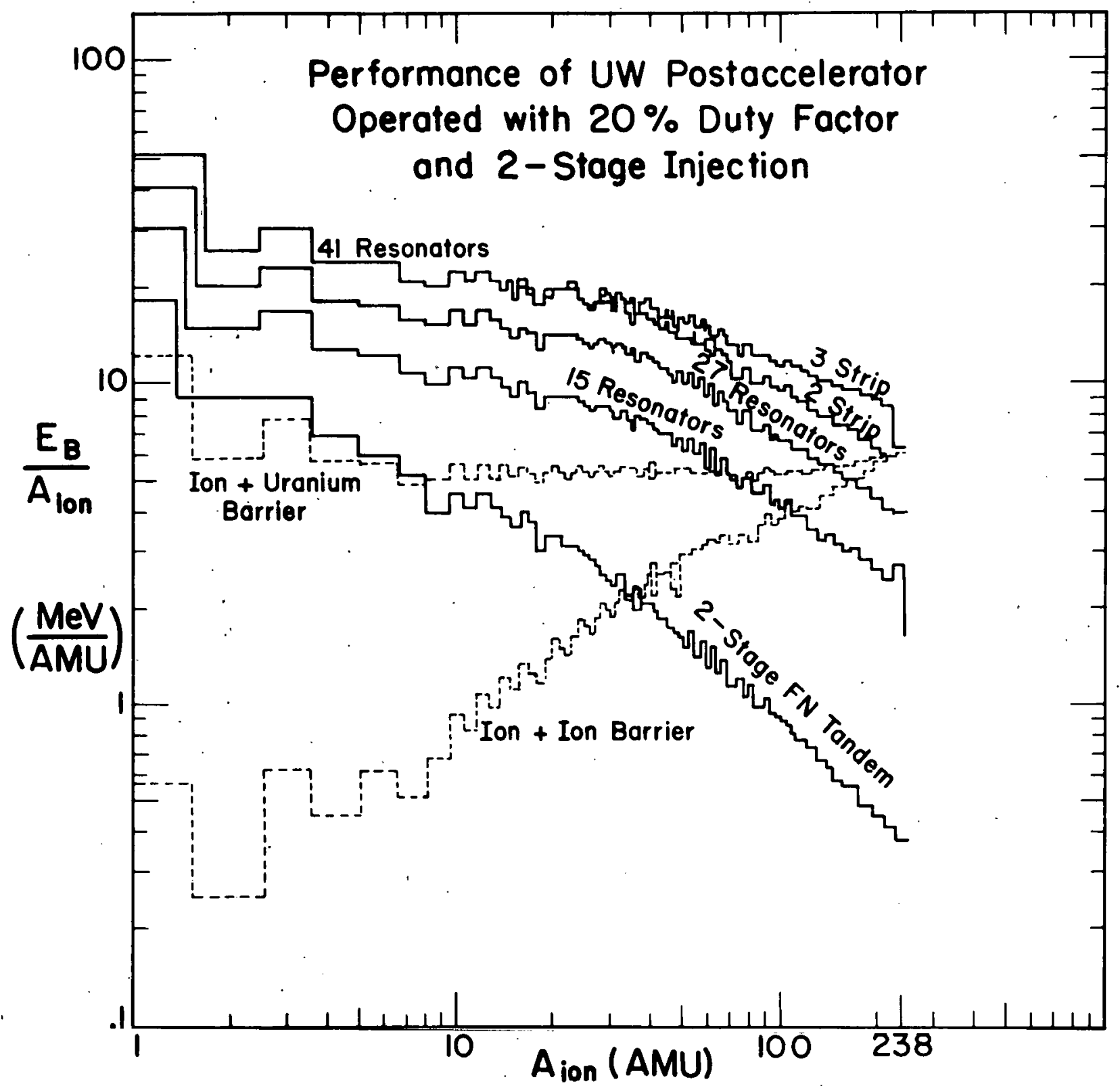

Fi.g. 1.5-3. Calculated energy performance of new postaccelerator design operated at $20 \%$ duty factor with beam injected from two stage FN tandem. Otherwise, curves are the same as in Fig. 1.5-2. 
Further it was found that a slightly larger and more powerful postaccelerator could be placed in the existing building by using a layout slightly different from that previously envisioned. The new layout is shown in Fig. 1.5-1. It requires two additional magnets but does not require the modifications of the existing magnets which would have been necessary under the old design. The physical sizes of the magnets shown are based on standard commercially available magnets.

Figure 1.5-2 shows the predicted performance of the redesigned postaccelerator injected by the three stage tandem and operated the $20 \%$ duty factor mode ( $20 \%$ on and $80 \%$ off at an average power of $20 \mathrm{~kW} /$ resonator). We have considered the performance of three configurations using 15, 27, and 41 resonators, respectively, to illustrate the modularity of the design and the excellent performance even in partially implemented configurations. In the 4 I resonator case we have considered operation with two foil strippings (tandem terminal and postaccelerator entrance) and three foil strippings (terminal, entrance, and before double $90^{\circ}$ deflection), the latter giving some enhancement in performance to the high-A beams. The RF power consumption of the postaccelerator is $820 \mathrm{~kW}$ (as compared to $1,280 \mathrm{~kW}$ for the old design) which represents an improvement in powor requirements and cost of about $2 / 3$. It should be noted that for the calculational results shown in this figure and the next we have used actual masses and chargcs of the stable isotopes. This results in fluctuations which reflect the actual fluctuations in the $Z / A$ ratio of the stable nuclides:

Figure 1.5-3 shows similar predicted performance when the postaccelerator is injected with beam from the two stage FN tandem without use of the injector tandem. This illustrates how gently the postaccelerator degrades when the energy of the input beam is reduced. Similar rather slight degradation is predicted when a gas stripper is assumed at the tandem terminal (although the recent discovery of a means of producing thin liquid films discussed in Sec. 1.8 shows promise that gas stripping may not be necessary). It should be noted in Figs. 1.5-2 and -3 that the performance of the preliminary configurations of the postaccelerator remain quite parallel to the 41 resonator curve, even in the high-A region. Thus the "crashes" referred to above have been eliminated and the utility of the preliminary configurations extended.

It should be mentioned that the above calculations assume that after each stripping the beam with the highest intensity charge state is used, and that by using weaker and higher charge state beams higher energies could be obtained. Further, we have assumed transit time factors based on sinusoidal field profiles. 4 However, with proper drift tube design it is possible to obtain 10 to $30 \%$ better transit time factors using more peaked field profiles. This would also result in improved accelerator performance beyond what was calculated here.

\footnotetext{
1. Nuclear Physics Laboratory Annual Report, University of Washington (1975), p. 6 .

2. A. Schempp, Optimierung von GSI-Rebuncher-Spiralresonatoren, Int. Rep. 75-9, Institut fur Angewandte Physik, Universitat Frankfurt/Main (1975).

3. Nuclear Physics Laboratory Annual Report, University of Washington (1975), p. 46 .

4. R.H. Stokes and D.D. Armstrong, Particle Accelerators (to be published).
} 


\subsection{Design of a Prototype Spiral Resonator Cavity}

\section{D.D. Chamberlin and J.G. Cramer}

A prototype spiral resonator cavity has been designed based on measurements of the model cavity (see Sec. 1.4) and is being constructed. The cavity is designed to hold a high vacuum $\left(10^{-7} \mathrm{~mm}\right)$, attach to standard beam lines, allow for cooling water flow within the structure and accommodate continuous input RF power levels in excess of $20 \mathrm{~kW}$ by tuning the input impedance and compensating for shifts in the resonant frequency.

Figure 1.6-1 shows the overall design of the prototype cavity. The main body is made from large diameter steel pipe electroplated with OFHC (oxygen free high conductivity) copper. The two end plates are of solid copper which is made to flow over a sharp $V$ edge of the steel tank to form a high quality vacuum seal. A removable hatch supports both the spiral structures which determine the resonant properties of the cavity and the coupling loop assembly through which the $\mathrm{RF}$ power is introduced. At the bottom is a vacuum port for connection to the pumping system and a large area compensator for frequency tuning of the cavity. Standard bolt circles on each end plate allow the cavity to be connected to standard beam lines.
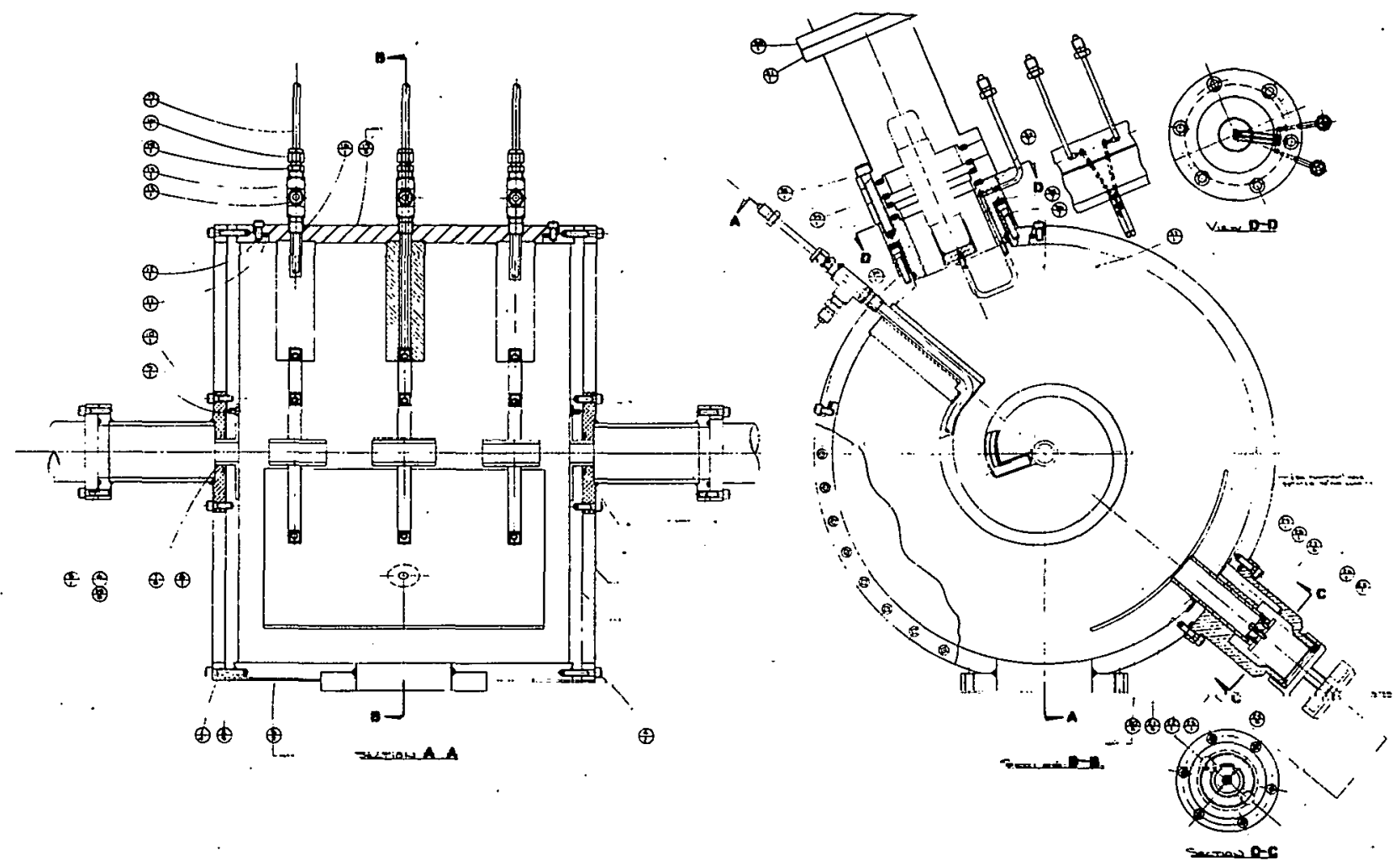

Fig. 1.6-1. Prototype Spiral Resonator Cavity. 
The removable hatch supports the thickened stems of the spiral structures. which terminate on the drift tubes. Increased surface area of the stems and of the square tubing of the spiral reduces resistance to the high $R F$ current flow in this region. An inner tubing provides for cooling water flow inside of each spiral. The length of the spirals has been chosen for the resonant frequency of the desired mode to fall at $109 \mathrm{MC}$, just above the commercial FM radio band. The geometry of the drift tubes was chosen to give a large value of the transit time factor for a wide range of particle velocities. Electrical contact between the hatch and the main body of the cavity is enhanced by spring fingers which bridge the gaps around the edges of the hatch. The hatch also contains the attachment point for external power sources and the coupling loop which transmits this power to the interior of the cavity. The coupling loop is a double section of copper tubing with cooling water flow and is fully rotatable in operation so that the input impedance of the system may be adjusted to a fully absorbing 50 uhins. The vacuum seal and exterior components are compatible with commercial FM transmission line equipment.

Under high power conditions the currents in the spirals give rise to distorting forces and resultant changes in the resonant frequency of the systeill. These changes may be offset by small motions of the compensator plate. This plate is externally adjustable through a bellows assembly and, although not currently water cooled, this could be accomplished with minor modification. With the exception of the small inserts at the beam entrance and exit, all interior' components are constructed of copper. These inserts, and perhaps similar inserts on the ends of the drift tubes, may be constructed of lighter elements to reduce the possibly very high $\mathrm{X}$-ray levels produced in the large electric fields. The interior surface of the tank itself is both machined and then further ground to give a smooth surface for the electroplated copper layer. Care with this surface and at the joining points of the structural elements is expected to enhance the shunt impedance of the cavity. Eventually other hatches and spiral structures will be constructed to explore the other operating modes of the cavity.

Early low power tests should determine an orientation for the coupling loop to allow smooth flow of the RF pnwer inte a $50 \mathrm{ohm}$ infut impeldarce. The neutral position of the compensator can be set to give easy tuning of the resonant frequency as the power level is increased. Low power measurements of shunt impedance and field profile can be used to predict acceleration properties of the prototype cavity in subsequent high power measurements. with light ion particle beams.

\subsection{A 1U8.6 $\mathrm{MHz}$ Buncher for 2- and 3-Stage Tandem Operation}

\section{D.D. Chamberlin and J.G. Cramer}

The bunching system described here is presently under design, and is intended to serve two rather different functions: (a) to producc high quality bunched beam on target for time-of-flight experiments with the 2 - and 3 -stage tandem, and (b) to provide high-quality high-transmission buncher heam for injcc tion of the postaccelerator described in Sec. 1.5 of this report. The latter function is very important because the beam quality delivered by the postaccelerator depends critically on the quality of bunched beam with which it is injected. 
If the injected beam is localized in a small time-energy phase space then the synchronous phase and time-energy optics of the postaccelerator can be adjusted to deliver an output beam which is bunched for excellent time resolution or debunched for excellent energy resolution. The transmission of the buncher is a multiplying factor in the transmission of the postaccelerator, so that a buncher system with high transmission is very important for good beam intensity from the postaccelerator.

In considering buncher design it is useful to realize that a buncher section is the time-energy analog of a focusing element, a chopper (deflection plate) section is the analog of a slit system, and that stripper foils broaden the angular and energy dimensions of a beam phase space distribution but do not alter the position and time dimensions of the phase space distribution. This means that to achieve optimum beam quality and transmission beam should be bunched and focused into a tight time and position focus at chopper and slit positions and at strippers.

A rather demanding requirement of the present system is that it be capable of bunching ions over the full range of available masses from $A=1$ to 238 , which implies a buncher phase velocity which can change by a factor of 16 and bunching structure sizes on the order of a few millimeters. 1 These requirements can, in principle, be eliminated by pre-accelerating the ions to an energy proportional to the ion mass $A$, so as to level the phase velocity and increase the size of the bunching structures. Unfortunately, this would require a variation of the preacceleration energy by more than two orders of magnitude and would also place unacceptable restrictions on ion source configurations. As will be discussed below, we have chosen instead a radical departure from the usual bunching technology by employing "traveling wave" bunching structures for the pre-acceleration buncher.

For experimental investigations involving time-of-flight (TOF) measurements, whether performed with the tandem alone or with the postaccelerator beam, it is important that the period between successive beam pulses include the flight times of all particles of interest. At an operating frequench of $108.6 \mathrm{MHz}$, as required by the postaccelerator, the period is only $9.21 \mathrm{nsec}$ which is not sufficient for many TOF measurements. Therefore a "weeding" system should be included as an integral part of the buncher to eliminate unwanted beam pulses and lengthen the time between pulses. The simultaneous requirements of "weeding" and high transmission pose special problems for the buncher design.

The above considerations represent a very demanding set of qualitative design criteria. The twin requirements of good time resolution and good transmission dictate that there should be two bunching systems, one before and one after acceleration. The TOF requirements dictate that the preacceleration system should be non-resonant so that it can function at sub-harmonics of the primary operating frequency of $108.6 \mathrm{MHz}$. The variable ion mass recuirements dictate that the preacceleration system should have a variable phase velocity which is tailored to the ion accelerated. Below we will discuss a preliminary buncher design which meets these criteria.

The Pre-Acceleration Bunching System. The pre-acceleration buncher consists of three principal elements, two bunching elements and a chopper. The first buncher time-focuses the beam to a sharp time peak at the position of the 


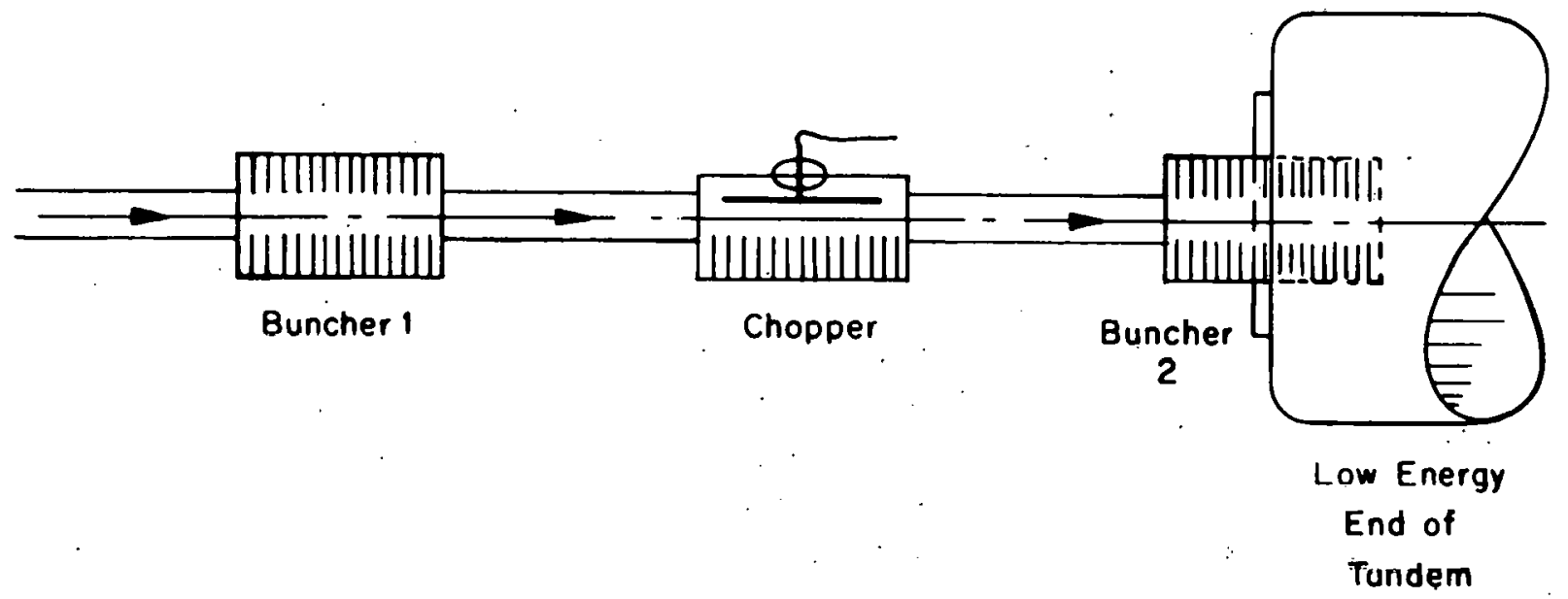

Fig. 1.7-1. Configuration of pre-acceleration buncher:

chopper. The latter acts as a set of time-slits, eliminating beam outside the time bunch by dispersing it radially so that it will not pass through the stripping aperture at the tandem terminal. The second buncher section rebunches the surviving beam so that it will arrive at the stripper in a tight time bunch. Figure 1.7-1 shows the bunching configuration at the low energy end of the two stage tandem. A single bunching element will also be placed in the terminal of the injector terminal after the scheme is tested in 2-stage operation:

The bunching and chopping elements of the pre-acceleration buncher are constructed as a set of parallel plate electrodes, each independently driven by its own driver electronics. Each driver module produces a parabolic bunching waveform, and the phasing of the system is controlled electronically. In addition, the plate electrodes are l).C. hiared so as to oimultaneously ach ds a focusing element. The system is nnn-rosonant and the frequency call be dicered

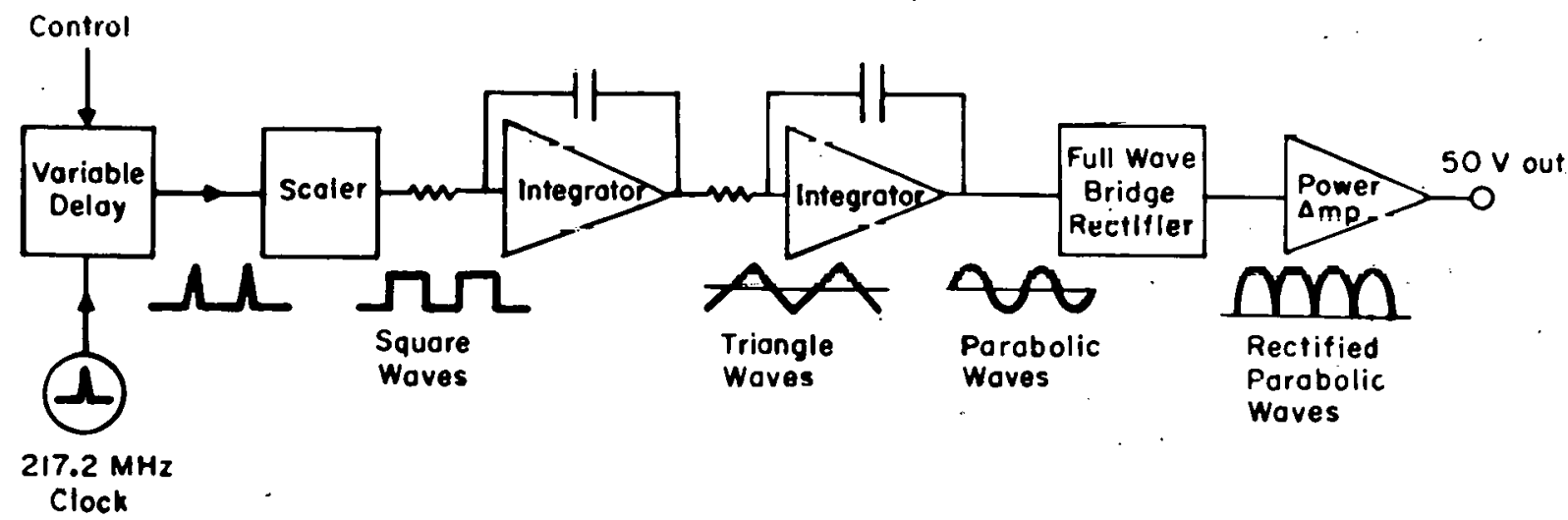

Fig. 1.7-2. Block diagram of driver module for pre-acceleration buncher. 


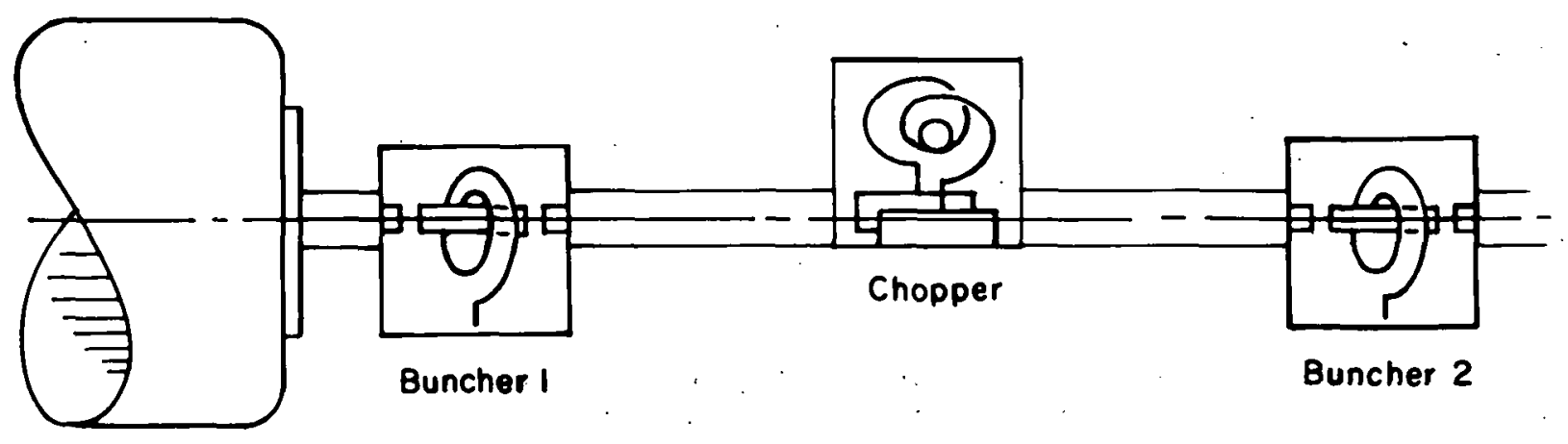

Fig. 1.7-3. Configuration of high energy buncher.

merely by changing the clock frequency.

Figure 1.7-2 shows a biock diagram of one of the driver modules. It is constructed using fast analog integrated circuits. The clock frequency can be made continuously variable, but in practice will be a $2^{\text {n }}$ scaledown from a 217.2 $\mathrm{MHz}$ crystal-controlled master oscillator. Both the buncher and chopper units use the same driver modules, but the buncher electrodes are annular discs with the beam passing through the axial hole while the chopper plates are rectangular, with their edges forming one plate of the pair used for chopping.

The High-Energy Bunching System. The bunching system at the high energy end of the tandem is in philosophy very similar to the pre-acceleration buncher described above. However, due to the bunching voltages required for accelerated beams, the elements of the system must be high efficiency resonant elements. The bunching elements are constructed using the spiral resonator technology described in Secs. 1.4-6 of this report. The chopper also employes the spiral resonator technology with a modified configuration such that the centers of the spirals are grounded while the stems support a pair of parallel chopper plates. This arrangement has been tested and found to give shunt impedances comparable to those of spiral resonator acceleration sections.

Figure 1.7-3 shows the high energy bunching configuration. The first element time-focuses the beam to a tight bunch at the chopper, where the second stripper unit is also located. The beam emerging from the chopper/stripper is rebunched by the second buncher unit so that it arrives at the entrance of the postaccelerator well matched to the time-energy phase space acceptance. of the postaccelerator. Consideration is currently being given as to whether the second buncher unit should inlcude a second-harmonic cavity. This appears to depend critically on the beam quality delivered by the preacceleration buncher after degradation due to transit time variations through the machine. Preliminary calculations indicate that the latter will not be so severe as to require harmonic bunching at the high energy end of the machine, but this is not a closed issue.

1. J.G. Cramer, Nucl. Instrum. Methods 128, 597 (1975). 
1.8 Production of Very Thin Self-Supporting Oil Films for Stripping and Target Applications

D.F. Burch, J.G. Cramer, and P.B. Cramer ${ }^{\dagger}$

The stripping of heavy ion beams plays an essential role in virtually all heavy ion accelerators and represents the limiting factor in many designs, yet there has been little progress in the development of new stripping techniques over the last decade. At present two types of strippers are in comnon use (a) gas strippers which can handle large beams but degrade the local vacuum and yield stripped ions with rather low average charge, and (b) solid foil strippers which give stripped ions with high average charge but are destroyed in a relatively short time by intense beams of heavy, ions and develop thickness variations due to differential radiation damage. Previous attempts to produce powder or droplet strippers have proved unsuccessful.l

The present work is an attempt to develop a new type nf striffer which combines the best features of the gas and solid stripping, sustaining high intensity beams without burnout while yielding stripped heams of high average charge. We have found that it is possible to produce stable free-standing films of diffusion pump oil through spinning the oil from the edge of a sharp-edged rotating disc. This produces an oil foil which is being continuously created so it cannot be burned out, and which has a density comparable to solids and should therefore yield stripped beams of high average charge.

We have studied the production of such films as a function of disc diameter and geometry, depth of disc-oil contact, disc rotation speed, oil type, and oil viscosity (through temperature variations). We find that it is extremely important to drive the disc in a vibration free way, and that a disc diameter of about $9 \mathrm{~cm}$ is optimum. The disc used was made of steel and the last half-centimeter of its radius was hollow-ground to a razon sharp edge. The disc was driven with a commercial Dumore hand grinder, operated with a triac variable speed control. The oil reservoir and spray shield were separately mounted on a screw jack so that the disc-oil contact depth could be precisely regulared. A dial gauge indicator was used to monitor this depth when the apparatus was in operation. Disc speeds were determined with a stroboscope tachometer. Dow Corning 200 fluid (viscosity $50 \mathrm{cp})^{2}$ at or slightly below room temperature was found to produce better films than other similar oils tested.

It was found that when the disc was driven at rather low speeds (500-1000 RPM) with rather deep contact depths, large stable oil films could be easily produced with surface areas of $10 \mathrm{~cm}^{2}$ or more. Figure 1.8-1. showa such a film. These films tend to be rather thick for use as strippers, with thicknesses of $1-100 \mathrm{mg} / \mathrm{cm}^{2}$ depending on the operating conditions. However, such films could serve very weil as targets. By using a colloidal suspension of some target material in a DC-200 carrier, it should be possible to provide burnout-proof targets of adjustable thickness for use in many types of nuclear and atomic physics experiments.

When the rotation rate of the disc was increased to 2500-3500 RPM and the contact depth was decreased to .1 to $.2 \mathrm{~mm}$, it was observed that a thin "connice" 


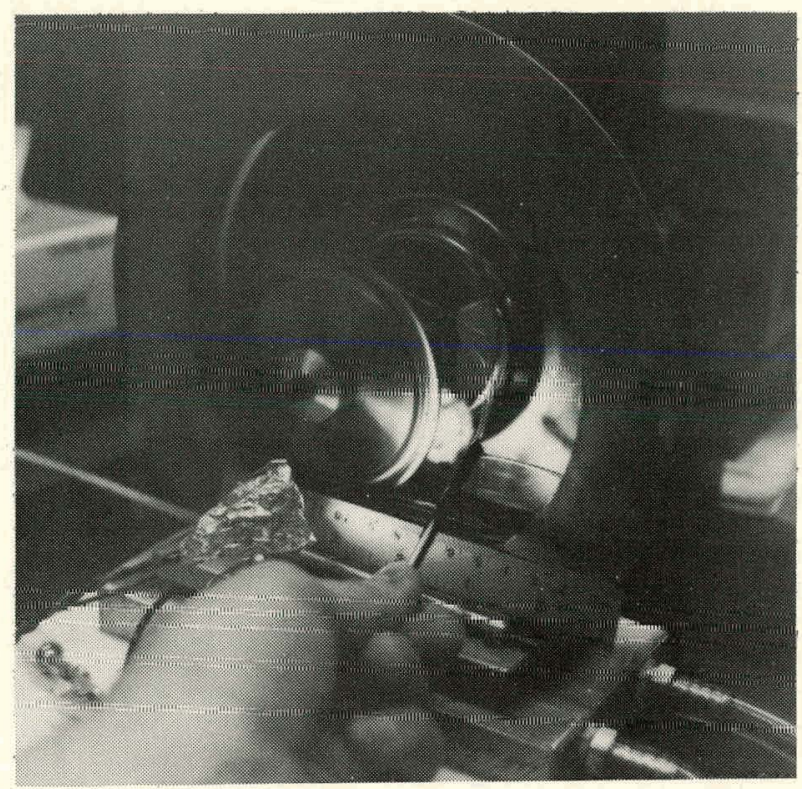

Fig. 1.8-1. Rotating wheel apparatus producing a thick oil film. Allen wrench inserted in the film breaks it into two streams, as shown.

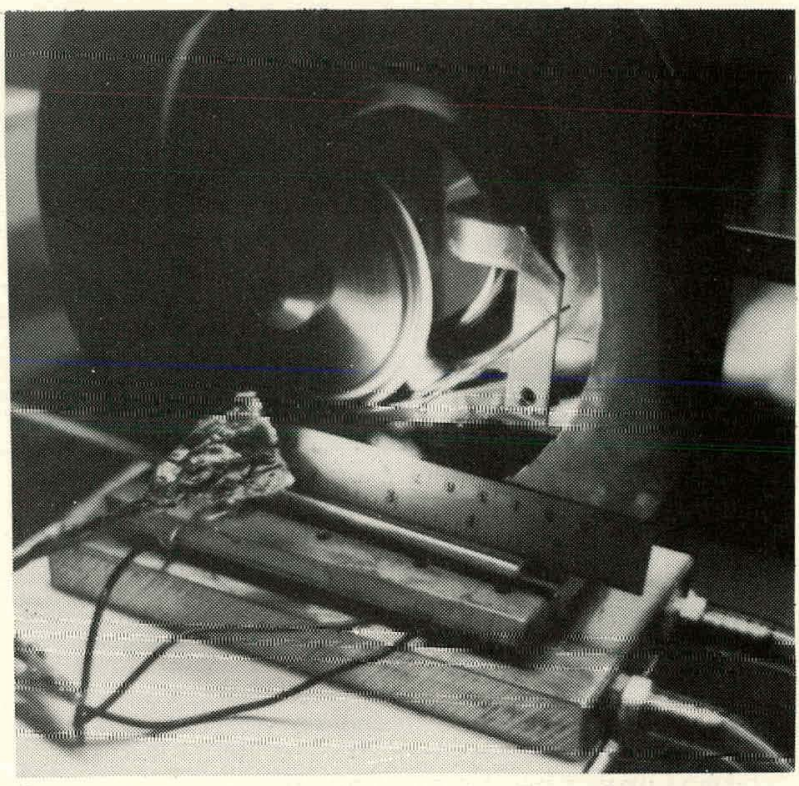

Fig. 1.8-2. Rotating wheel apparatus with scraper producing thin oil film which is illuminated by small incandescent lamp. Color bands are visible in film near edge of wheel.

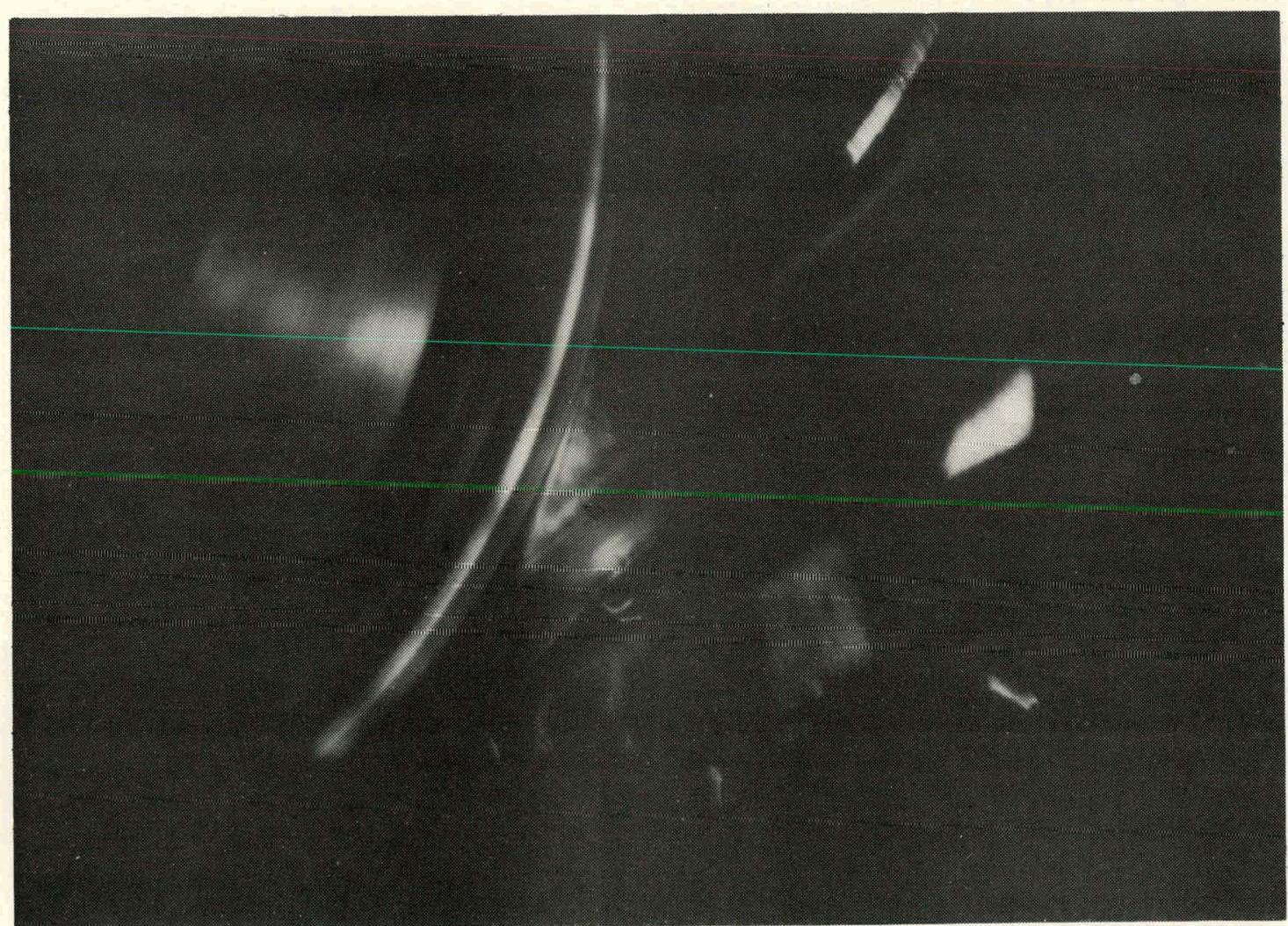

Fig. 1.8-3. Closeup view of thin film illuminated with a He-Ne laser. Monochromatic light forms bright and dark interference bands in the thin film, permitting estimates of its thickness and density profile. 
film formed at the edge of the disc in which interference colors were visible. Such a film is shown in Figs. 1.8-2 and -3 . Fig. 1.8-3 was made using a Laser monc chromatic light source, so the interference pattern shows up as alternating bright and dark bands. Careful adjustment of the speed and contact depth allowed the production of such films with thicknesses of 10 to $20 \mu \mathrm{gm} / \mathrm{cm}^{2}$ and areas of essentially uniform thickness of about 8-10 $\mathrm{mm}^{2}$ and triangular in shape. The above thicknesses are determined from the interference pattern produced by the films when viewed in white light. It was found that even thinner films could be produced by bringing a "scraper" surface almost in contact with the edge of the disc. This has the effect of increasing the gradient of shear forces in the fluid, reducing the amount of oil picked up on the edge of the disc and providing a supporting lower surface for the film produced. Films produced with a scraper as shown in Fig. $1.8-2$ have been observed with thicknesses of about $5 \mu \mathrm{gm} / \mathrm{cm}^{2}$.

Up to this point we have demonstrated the production of very thin films of diffusion pump oil which are, in principle, suitable for stripping foils. We have yet to demonstrate that the same films can be produced in vacuun, that the optinal thickncss measurements can be verified by cnergy luss measurements with an alpha suurce, that the technique can be reliably implemented to produce a practical stripping device which can be operated at the terminal of a tandem or elsewhere, that there is not undue degradation of the oil due to direct bombardment by a beam, and that a liquid stripper gives the same high average charge as a solid stripper.

1. E.H. Bothur, G. Clausnitzer, and E. Salzborn, Nucl. Instrum. Methods 121, 533 (1974); K. Purser (private communication).

2. Available from Dow-Corning Corporation.

1.9 OSHA-WISHA Compliance

W.G. Weitkamp and Staff

The State of Washington implementation of the Federal Occupatinnal Safcty and Health Act, referred to as WISHA, Washington Industrial Health and Safety Act, became effective June 7, 1973. Since that time the Laboratory has maintained an active program correcting deficiencies as they are identified. This is, of course, to be expected, but it became apparent during the past year that identifying and correcting WISHA violations will require a continuing effort, involving a substantial fraction of Laboratory stalf time.

Sec. 1.2)

Among the deflciencies corrected during the past year are: (see also

1. The ventilation for the machine shop welding station was found to be inddequate. An engineering study of the possible corrective action revealed that the electric power service installed in the shop building in about 1950 was not only inadequate to handle additional ventilation equipment, but was overloaded with existing machinery. With the aid of a grant from the University of Washington, a new building service and a WISH $\Lambda$ standard ventilation system were installed. 
2. Work is underway to modify the more than 50 machine tools in the Laboratory to meet WISHA standards for guards and interlocks on access doors.

3. Low usage machine tools have been disposed of to provide adequate clearance around remaining machines.

4. Several machine shop units such as the blast cabinet in the student shop have been replaced because of the high cost of bringing the old units into compliance.

5. A permanent cage has been built around the high voltage power supplies for the ion source test stand.

6. Maximum floor loadings have been determined and posted. Stair railings and walkways have been corrected, and metal storage fixtures strengthened and guarded where necessary.

7. Storage areas for radioactive materials have been cleaned up, and unneeded materials disposed of. 


\section{ION SOURCE DEVELOPMENT}

2.1 Polarized Ion Source Development

E.G. Adelberger, W.B. Ingalls, C. Sum, H.E. Swanson, and T.A. Trainor

Implementation of the design program described in last year's Annual Reportl has been completed. The overall system is depicted in Fig. 2.1-1. Target proton current is presently 100-140 nA with a polarization of 0.73. Deuteron target currents are in the range $150-200 \mathrm{nA}$ with vector polarization $\mathrm{p}_{z}=0.50$ and tensor polarization $\mathrm{p}_{z z}=0.60$. This represents a factor of 10 increase in source output since new construction began.

A calibration curve fur the new Wien precessor is shown in Fig. 2.1-2. This curve was obtained with a tensor-polarized deuteron beam and a recently constructed tensor polarimeter as diccussed in Ser. 11.1. of this i'epurt. Saturation in part of the magnetic circuit limits the maximum (1200 eV) deuteron spinrotation angle to $125^{\circ}$. More extensive use of higher permeability magnetic material in the flux-return path would increase this angle to $150^{\circ}$. However, we have no need for rotations greater than $90^{\circ}$ at present.

The rotational position of the precessor is determined by a stepping motor. This is presently controlled at ground potential by a single two-way switch and light-pipe system. However, a digital control system is being

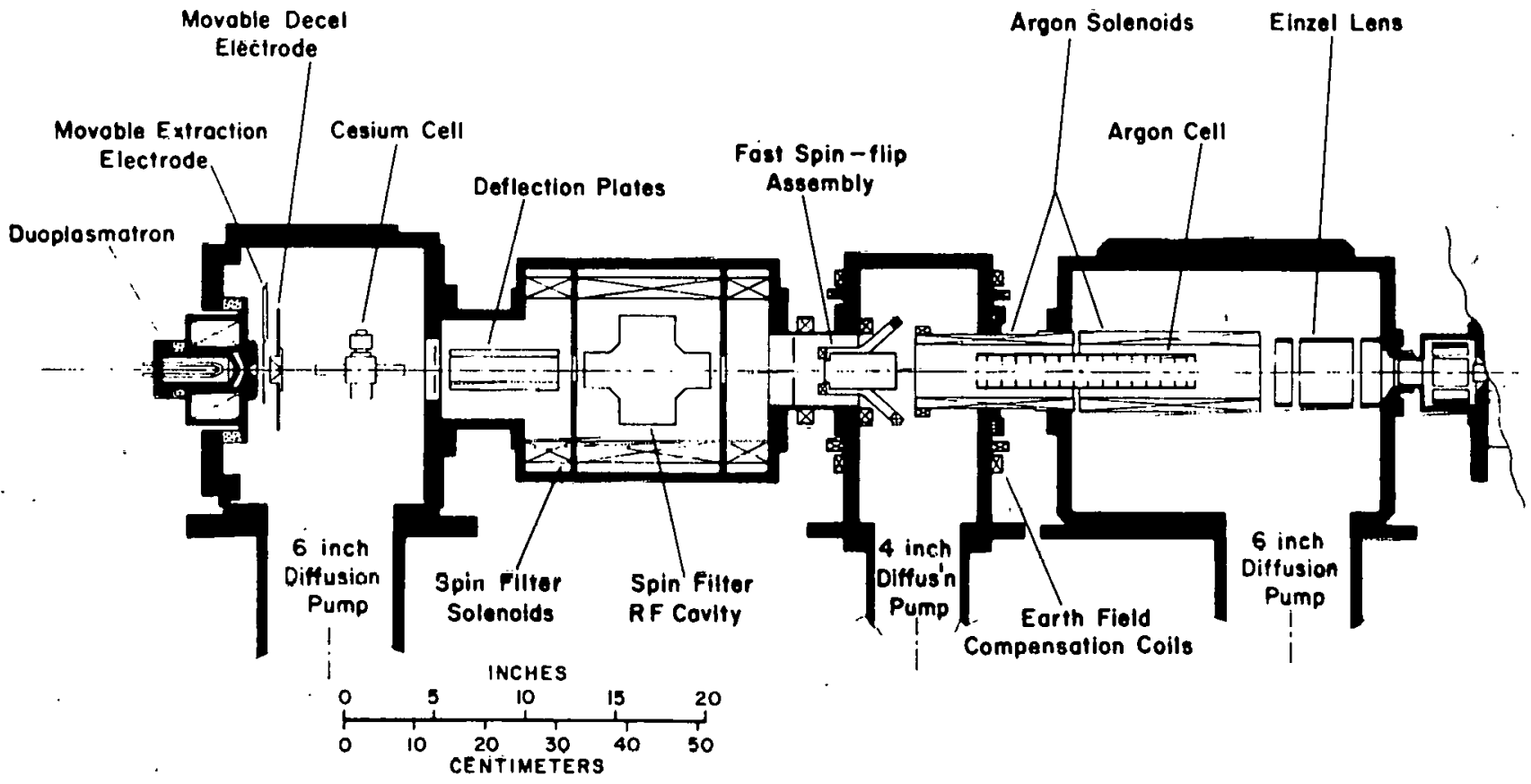

Fig. 2.1-1. Polarized Ion Source. 
developed which will make it possible to accurately position the precessor as part of a feedback loop which receives information from a helium polarimeter at the target (Sec. 3.8). The position and field strengths of the precessor are transmitted to ground potential by means of a voltage-to-frequency converter and light-pipe system.

Degradation of beam quality by the precessor appears to be minimal. For example, at maximum field strengths the spin of a $600 \mathrm{eV}$ proton beam rotates through $800^{\circ}$. The reduction in beam intensity is less than $5 \%$ and the depolarization is on the order of $3 \%$. At normal field values, therefore, the precessor has a negligible effect on beam quality.

Figure 2.1-3 shows calculated and measured magnetic fields and calculated electric fields inside the precessor. In general the fields are uniform across the beam diameter to about $5 / 10^{4}$ or less and the electric and magnetic fields are quite well matched along the beam axis.

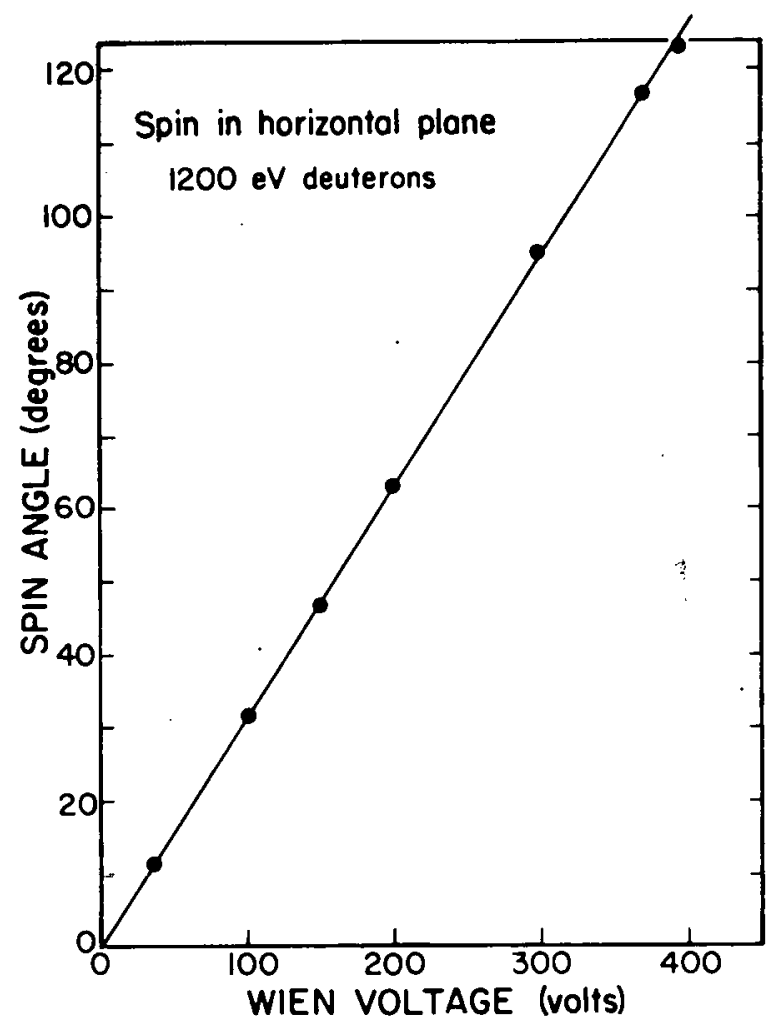

Installation of the new acceleration tube has eliminated voltage-breakdown problems in this area and has improved beam-optical quality. The variable crossover aperture in this device is an ideal place to reduce beam in cases where an experiment is count-rate limited. However, beam-polarization enhancement by scraping on this aperture is not a large effect in this source for two reasons. On the one hand, use of the Sona polarization method requires a large ionizing field at the argon cell, resulting in significant emittance degradation. The additional emittance seems to be comparable to or larger than the emittance difference between negatives from metastable and ground-state atoms upon which the beam-scraping enhancement relies, thereby reducing the effect. In addition, the spin precessor and an einzel lens are located upstream from the crossover aperture and may further "mix" polarized and unpolarized components. In any case, polarization enhancement is only a few per cent in the case of protons and vectorpolarized deuterons and about $5 \%$ in the case of tensor-polarized deuterons. For normal operation the crossover aperture diameter is 100 or $150 \mathrm{mils.} \mathrm{With} \mathrm{in-}$ stallation of the spin filter described below the polarization enhancement is expected to be larger

The largest single increase in beam intensity was obtained with installation of the "argon box" vacuum enclosure. Prior to this installation, target proton currents were typically 30-50 nA with a polarization of $65 \%$. At times the polarization dropped as low as $50 \%$, usually with accompanying loss of beam. These aperiodic variations in beam quality were not correlated with any monitored 


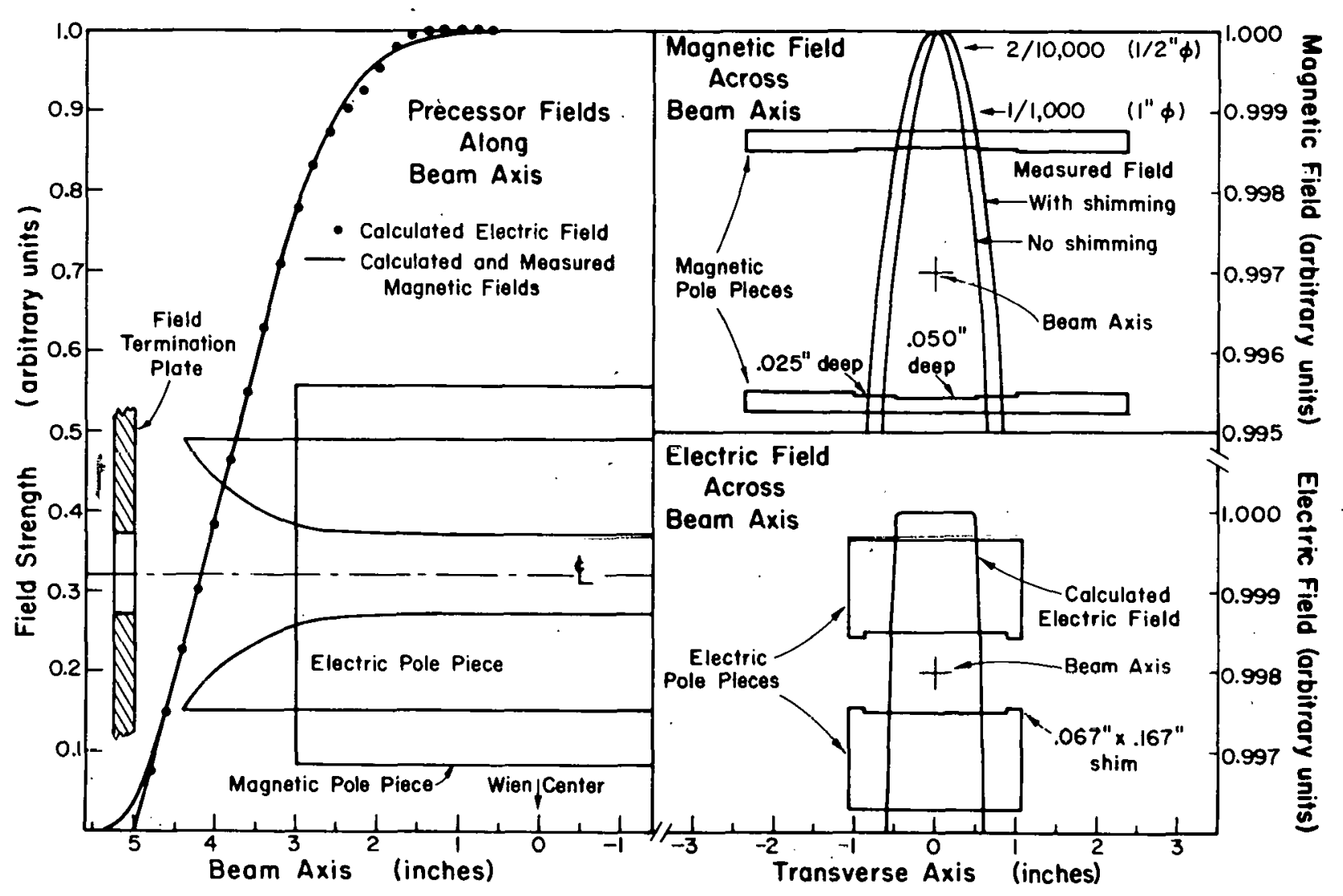

Fig. 2.1-3. Spin precessor fiields.

source parameter, but the cause was isolated hetween the Sona crocoover and the spin precessor. Ironically, this situation worsened as the positive ion zource and operating pressures were improved. The expected improvement in beam intensity with installation of the new box was $50-70 \%$, based on the $60 \%$ transparency of the old multicapillary argon cell, small-diameter einzel lens and poor gas pumping of the original system. In fact, beam intensity increased by a factor of 3-4 and the polarization is now $73-75 \%$. These figures, in addition to quench ratios taken at various times, are consistent with a loss of as much as $50 \%$ of the metastable beam in the region of the argon cell with the old system. We speculate that some form of quenching was associated with intense metastable beam impinging on the leading edges of the capillary tubes in the argon cell. This situation seemed to worsen with more intense beams. Because of this experience we have reduced collimation of metastahle beam to a minimum and have precedelt remaining collimators with tapered tubes to shield the beam from any charge build-up.

Because of a major reorganization of the hardware on the low-energy end of the tandem accelerator planned in the near future, the Laboratory will be willoul the services of the He-Li charge exchange source for up to a year. For this reason we decided to investigate the possibility of producing helium beams with the polarized source. To. this end we purchased a $4 \mathrm{kV}, 50 \mathrm{~mA}$ beam-energy supply 
during the ion source upgrade described above. We find that a Lamb-shift source is a more-than-adequate source of helium beams, using the double charge exchange of helium on cesium to produce $\mathrm{He}^{-}$. With no alteration of ion-source hardware we have produced beams of 5-6 $\mu \mathrm{A}{ }^{4} \mathrm{He}^{++}$after energy analysis and a target current of $4 \mu \mathrm{A}$ of $9 \mathrm{MeV}^{4} \mathrm{He}^{++}$on target during a recent $(\alpha, \gamma)$ experiment. The corresponding ${ }^{4} \mathrm{He}^{-}$current at the low-energy end of the accelerator was $5 \mu \mathrm{A}$.

Charge exchange with cesium took place at a helium ion energy of $2900 \mathrm{eV}$. This figure (an upper limit) was determined by the focusing ability of the positive ion source accel-decel system and the first gap lens in the $50 \mathrm{kV}$ acceleration tube. Higher positive ion energies would require modification of the acceldecel system to provide stronger focusing of the extracted beam and purchase of a higher voltage supply for Gap l. For this reason we cannot say what the optimum energy is for this charge-exchange process. However, we are quite satisfied with the present results. Aside from the higher positive beam energy, operation with helium requires only turning off the magnetic and electric fields associated with the polarization process for proton and deuteron beams. The cesium cell operates at a slightly higher temperature $\left(130^{\circ} \mathrm{C}\right.$ rather than $\left.110^{\circ} \mathrm{C}\right)$ and the arc current is somewhat lower ( $6 \mathrm{~A}$ rather than $9 \mathrm{~A}$ ). The oxide-coated mesh filament must be raised from $25 \mathrm{~A}$ (normal) to $30 \mathrm{~A}$ to maintain a stable arc. Helium pressure in the anode region is 0.3 torr (uncorrected thermistor gauge calibrated for air). Extracted positive He beam is $3.5 \mathrm{~mA}$.

We have given thought to the possibility of installing a ${ }^{3} \mathrm{He}$ recovery system on the polarized source. Such a system would cost about $\$ 1500$ and would fit in presently available space on the ion source frame. Cost of $3_{\text {He operation }}$ is estimated (at preserit ${ }^{3} \mathrm{He}$ prices) to be $\$ 60 / \mu \mathrm{A}-$ day. Implementation of the recovery system would depend on projected use of ${ }^{3} \mathrm{He}$ beams and a schedule of construction of a separate helium source.

The potential of the He-Cs charge exchange reaction for production of helium beams appears to be tremendous. It should be noted that the path length between production of $\mathrm{He}$ at $2900 \mathrm{eV}$ and acceleration to $50 \mathrm{kV}$ is more than $2 \mathrm{~m}$ in the polarized source whereas typical length of a He-Li source is less than $0.6 \mathrm{~m}$. It is quite possible, therefore, to expect that a He-Cs source could be designed to deliver negative helium beams of $30 \mu \mathrm{A}$ or more.

We have recently decided to construct and install a spin filter to replace the Sona polarization scheme presently in use on the source. The spin filter, developed by McKibben and coworkers at Los Alamos Scientific Laboratory (LASL) has several clear advantages over the Sona scheme, chiefly the ability to determine the absolute beam polarization by a single source diagnostic and greatly enhanced deuteron beam polarization. In addition, it makes possible the ability to flip the spin direction at a high rate with spin-correlated beam motion several orders of magnitude less than our present fast-flip method. This is of great importance for our continuing efforts to improve the $19_{\mathrm{F}}$ parity-violation experiment.

The recently completed source upgrading described above was pursued with the intention that a spin filter might ultimately be installed. Therefore, all existing power supplies and hardware, with the exception of two 575G solenoids, are compatible with the spin filter installation. The polarized source with 
spin filter is shown in Fig. 2.1-1. The fast spin-flip assembly follows the design of McKibben and Potter ${ }^{2}$ and the spin filter assembly is of the new "short" design developed by Hardekopf for the LASL polarized triton source. 3

A prototype RF cavity has been constructed for use in testing the RF system as it is constructed and fabrication of the spin-filter solenoids has begun.

1. Nuclear Physics Laboratory Annual Report, University of Washington (1975), p. 18.

2. J.L. McKibben and J.M. Potter in Proceedings of the Fourth International Symposium on Polarization Phenomena in Nuclear Reactions, 1975, to be published.

3. R.A. Hardekopf, Ibi.d.

2.2 Sputter Iöil Bunice

G. Roth and J. Wiborg

The sputter ion source purchased from Extrion Corporation has undergone major revisions in the past year. Initial use of the source was beset with a number of problems such as severe sparking, ionizer burnout, ionizer heater burnout, and unstable beam output. Early in the past year the source was taken off the accelerator and run on the test stand to correct these problems. It became evident that all these problems were related to failures of the cesium ionizer and boiler seals. These are crushable metal seals. Boiler seal failure would allow air into the cesium reservoir, making the beam unstable. Ionizer seal failure would allow cesium to leak into the extractinn region causing the ecverc sparking. Also, cesium would short the decel electrode designed to prevent backstreaming beam from striking the ionizer and burning a hole in the tip. The entire boiler and ionizer assembly was rebujlt using varian mini-conflat soals and has been extremely successful.

Several other problems have been corrected during the shutdown to improve reliability. The gas line connection was changed to a more reliably leak-tight fitting. The decel electrode high voltage feedthrough was changed to provide better insulation. The decel electrode insulator was shielded by a sleeve to prevent cesium from coating and shorting the insulator. New ionizer heater lead clamps were installed which can be tightened without twisting and pulling the leads out of the heater. The boiler heater power supply was originally supplied by Extrion with a solid state temperature regulator. Sparks caused frequent failure of this device. The entire supply has been replaced with a non-regulated supply which seems quite adequate. The $30 \mathrm{kv}$ extraction supply has been changed from an air insulated to an oil insulated supply which results in a much more rugged unit capable of withstanding severe sparking without damage.

An Einzel lens has been added to focus the cesium beam onto the cone. This has not been fully tested yet but its effect seems to be greatest for hard to sputter materials. The cesium can be focused to a small spot right at the cone exit hole and in some cases a $25-50 \%$ beam increase can be seen. 
A still unsolved problem is the very poor transmission of sputter source beams through the Van de Graaff. The transmission gets lower as the ion mass increases indicating not only poor emittance but a poor optical match between the accelerator entrance and the low velocity beams. A possible solution is to raise the entire source to a potential of $50-100 \mathrm{kv}$. A new vacuum stand is being constructed for the sputter source and the possibility of electrically floating the source is being incorporated.

The source is now back in operation and has run briefly for experiments with indications of much greater reliability and with beam output about $25 \%$ greater than before the modifications. 


\section{INSTRUMENTATION, DETECTORS, RESEARCH TECHNIQUES}

\subsection{Silicon Detectors}

\section{S. Kellenbarger}

We continue to make our own lithium-drifted detectors, both circular for general use and rectangular-shaped for use in the "sideless" mounts. We also make rectangular surface barrier detectors to use in the "sideless" mounts for detecting heavy ions. Our efforts during the past year have been directed toward maintaining our stock of these detectors. No significant changes have been made in our production methods since our 1975 Annual Report.

\subsection{Further Development of a Gas Cell-Detector System}

Y-d Chan, J.G. Cramer, K-L Liu, and B.A. Scott

A large solid ângle gàs cell-detector system has been constructed, and was described in last year's Annual Report.l The system is designed to measure reaction cross sections tor gas targets with high efficiency. It can also be used as a Q-window device to select specific channels for coincidence measurements. A schematic drawing illustrating the detection method is shown in the insert of Fig. 3.2-1. Further details are given in Ref. 1.

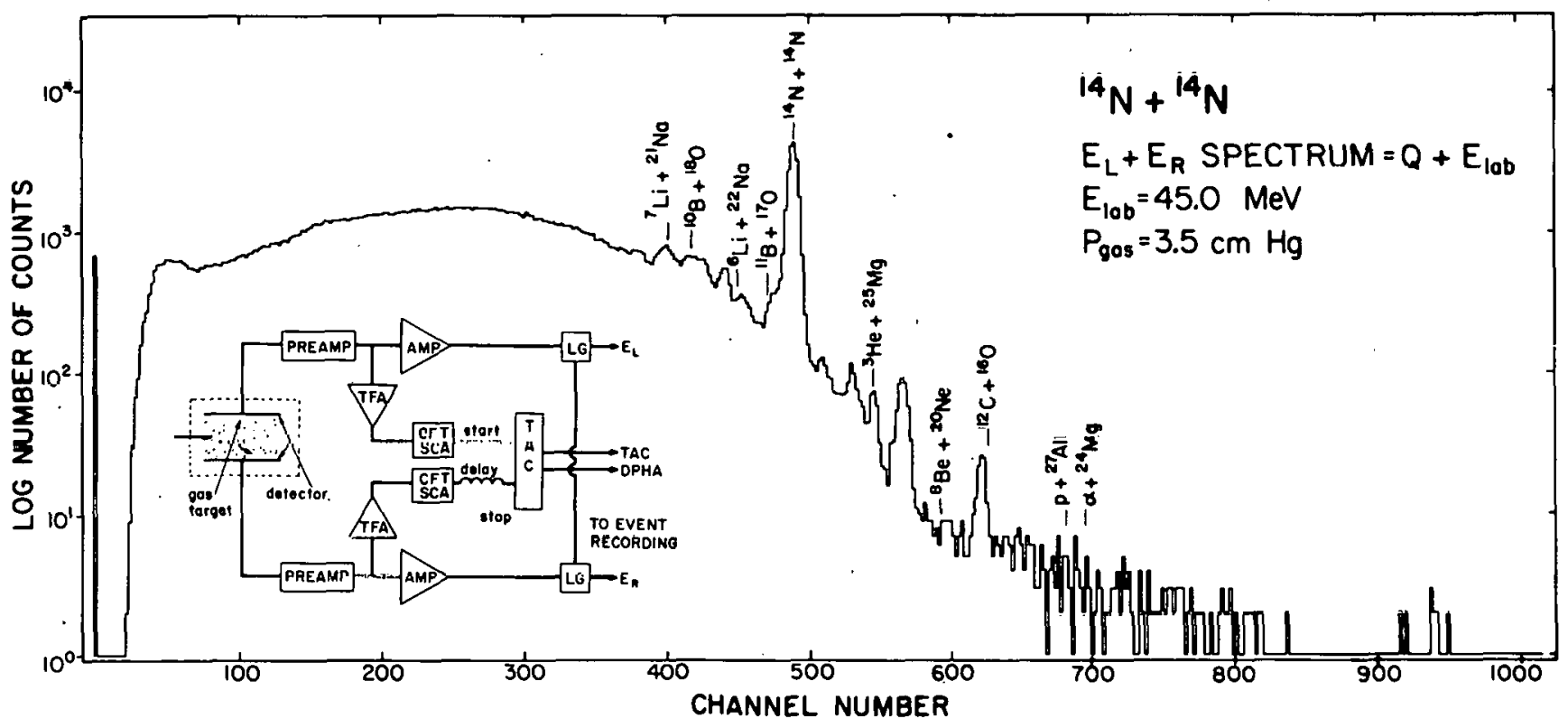

Fig. 3.2-1. $E_{L}+E_{R}$ spectrum $\left(Q+E_{l a b}\right)$ for $E_{l a b}=45.0 \mathrm{MeV}{ }^{14} \mathrm{~N}$ on ${ }^{1 \prime \prime} \mathrm{N}$. The calibration is given by $Q+E_{l a b}=(0.0769 *$ (channel no.) +6.927$) \mathrm{MeV}$. Vertical marks indicated the location of some known channels. The insert is a schematic drawing of the gas cell-detector system and the electronics used during the test run. 
In order to improve the performance of the above system, the following features have been added:

(1) Two extension barrels with adjustable aperture pieces were added, to reduce multiple scattering events inside the gas cell.

(2) Two insulated cument-readout pieces were added next to the entrance and exit windows of the cell to monitor the center position of the beam.

A test run has been carried out with a ${ }^{14} \mathrm{~N}$ beam bombarding a ${ }^{14} \mathrm{~N}$ gas target. A summed spectrum $E_{L}+E_{R}\left(=Q+E_{l a b}\right)$ for $E_{l a b}=45.0 \mathrm{MeV}$ is shown in Fig. 3.2-1.

Discussion of Results

Calibration of the spectrum was done by changing the incoming beam energy $E_{l a b}$. From the corresponding shifts of the $Q=0$ peak channel, one can have an absolute calibration of the spectrum. Five different energies $(40.0-45.0 \mathrm{MeV})$ were used to obtain the calibration parameters.

As can be seen from Fig. 3.2-1, there is a discrete and well defined peak structure in the spectrum, with the strongest peak being identified as the elastic channel $(Q=0)$ peak.

However, Q-values obtained from our calibration cannot be matched satisfactorily with known reaction channels, except for several cases. The matching of the $12 \mathrm{C}+16 \mathrm{O}$ and ${ }^{3} \mathrm{He}+25 \mathrm{Mg}$ channel is quite satisfactory if one also considers the energy loss of the particles inside the gas target. (See Fig. 3.2-1 for the comparison of known channels and the location of peaks in our spectrum.) Very light particle channels were not reliably measured at the time of this test run, due to the limited thickness of our solid state detectors: The best energy resolution we achieved in the combined Q-spectra is about $420 \mathrm{keV}$ (FWHM) for the $\mathrm{Q}=0$ peak. This width is probably due to the different path lengths in the gas target.

A problem from high background still exists, which possibly can be improved only by expanding the dimensions of the gas cell. Also, it is planned to put foils of different thickness in front of the detectors to help in identifying the peaks. For the time being, due to the only fair resolution in the Q-spectra, this device is not yet ready for actual experimental applications.

1. Nuclear Physics Laboratory Annual Report, University of Washington (1975), p. 25 .

\subsection{Gas Ionization Counter Telescope}

R. Bangert, J.G. Cramer, C.K. Gelbke, and K-L Liu

With the production of various heavy ion beams, such as Al and Si, from the sputtering source, 1 it becomes apparent that a particle identification telescope for slow nuclei with $A>1 \hat{b}$ is necessary. ${ }^{l}$ The conventional solid state 


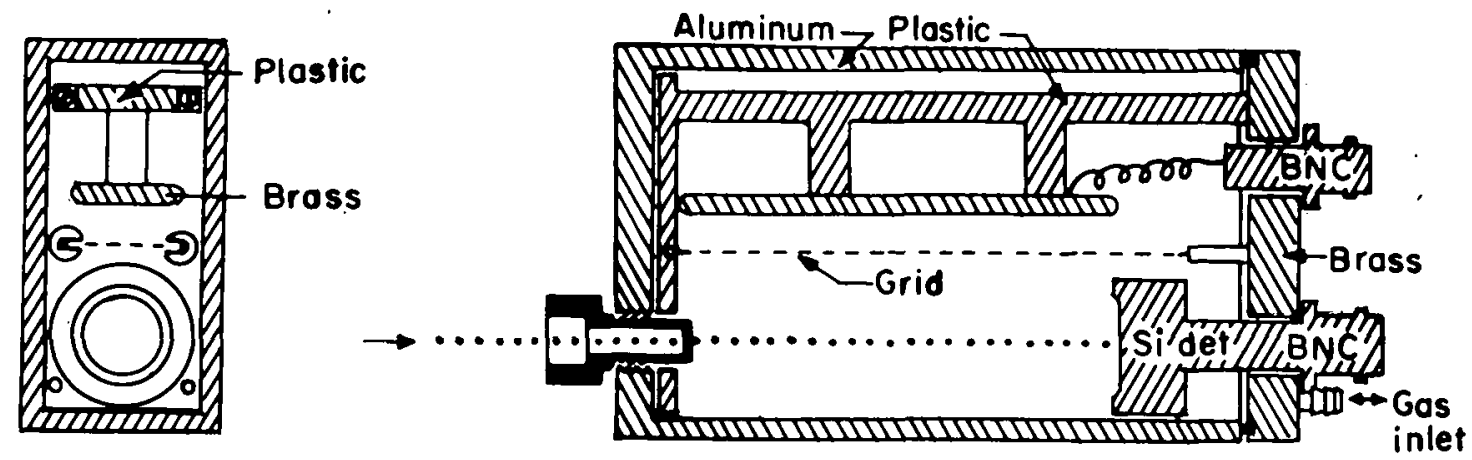

Fig. 3.3-1. A cross-sectional view of the gas ionization telescope.

transmission detector is too thick for slow particles of this mass lu pass through, so a gas ionization counter was developed as a transmission detector. This device has the advantages of small thickness which can be varied by adjusting the gas pressure, and of good thickness uniformity. The effective thickness of the device is essentially that of the entrance window.

The ionization counter telescope system shown in Fig. 3.3-1 is quite similar to one designed at the Lawrence Berkeley Laboratory, with some minor modifications. 2

The housing of the counter is made from rectangular aluminum tubing with another aluminum plate welded to the frent. On this dlumlnum plate, a $1.2 \mathrm{~cm}$ screw hole is tapped for mounting the entrance window. The back of the ionization chamber is covered with a brass plate sealed to the housing with an 0-ring. The grid, the gas inlet and the solid state stopping detector are mounted on this brass plate. The grid is made from nickel mesh of about $98 \%$ transparency. The ion entrance window is made out of VYNS foil which can be made to an area density of $10 \mathrm{\mu g} / \mathrm{cm}^{2}$ with a uniformity of about $\pm 5 \%$.

An ionization chamber used as a transmission detector requires constant pressure to give stable particle identification. A Cartesian manostat has been employed for this purpose. The gas supply and regulation system is shown in Fig. 3.3-2. With this setup, it is found that the preesure is stable to within $\pm 0.5 \%$ uver a period of three days.

The telescupe has been found to be useful for change separation of $S i$ and A. down to about $25 \mathrm{MaV}$ of the detected particle.'J

1. See Sec. 2.2 of this report.

2. M.M. Fowler and R.C. Jared, Nucl. Instrum. Methods 124, 341 (1975).

3. See Sec. 12.7 of this report. 


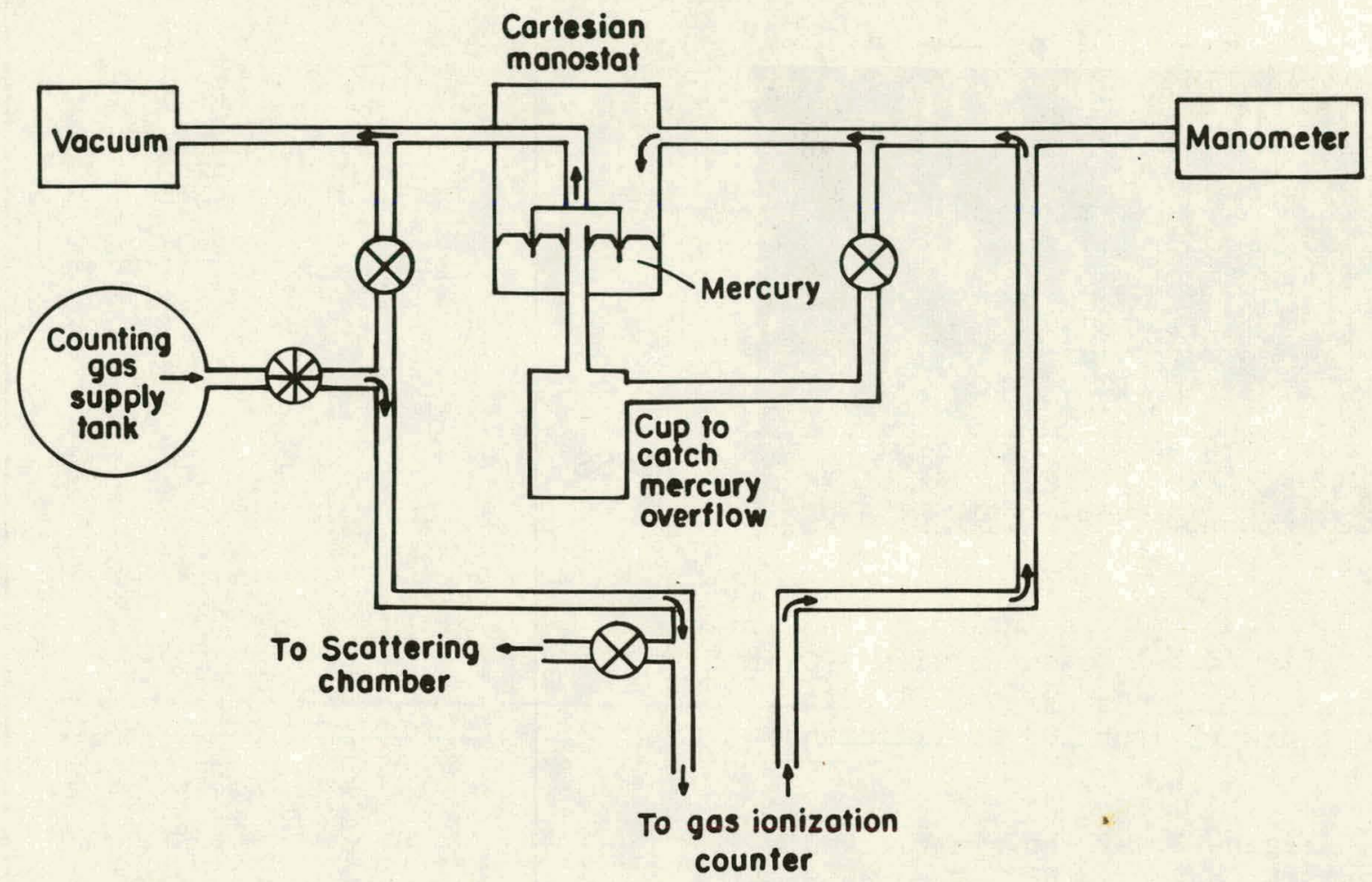

Fig. 3.3-2. Diagram of gas supply and regulation system. indicates gas leaking valve.

3.4 A Permanent Electronics Setup for High Resolution Timing and Spectroscopy

R. Bangert and $\mathrm{K}-\mathrm{L}$ Liu

We have initiated the use of a high resolution system for timing and spectroscopy with semiconductor detectors. Its setup is easy and its performance is reproducible without any problems. It allows time-of-flight or coincidence measurements of heavy ions with a time resolution better than 120 ps and give a negligible noise contribution to the energy spectra.

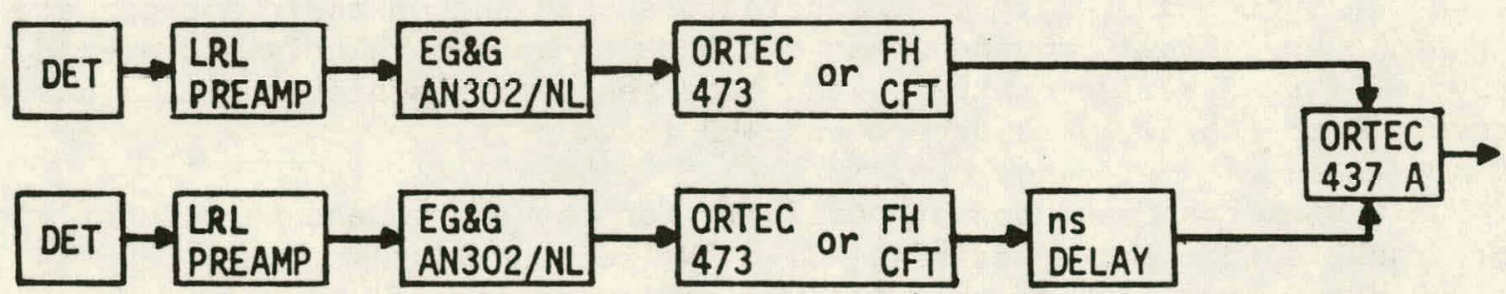

Fig. 3.4-1. Electronics setup for fast timing between semiconductor detectors. 


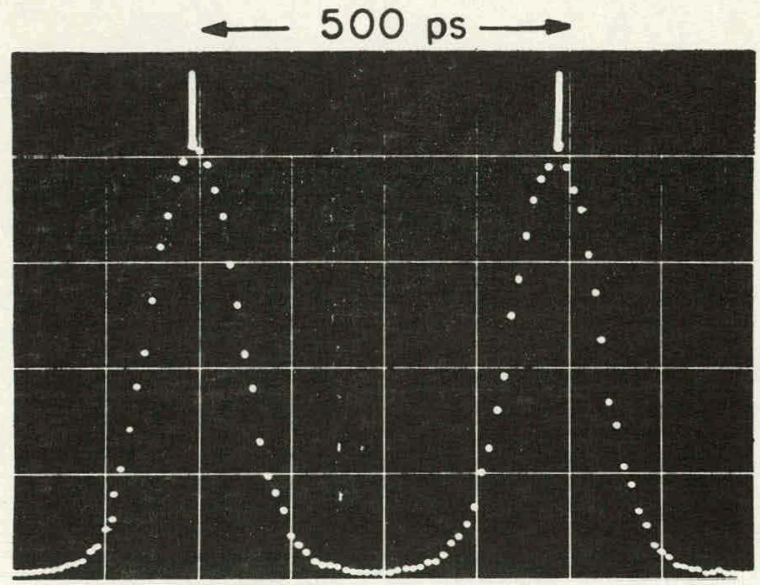

Fig. 3.4-2. Time resolution of the system in Fig. 3.4-1 measured with 160 at $30 \mathrm{MeV}$ incident energy. $\triangle \mathrm{E}-\mathrm{DET}=10.5 \mathrm{u}$, $25 \mathrm{~mm}^{2}, \mathrm{E}-\mathrm{DET}=10 \mathrm{lu}, 100 \mathrm{~mm}^{2}$, distance $13 \mathrm{~mm}$.

Figure 3.4-1 shows a block diagram of the basic setup. The essential characteristics are: Preamplifier: $\tau_{\mathrm{r}}^{\text {fast }}=1.2 \mathrm{~ns}$ with open input, $\tau_{\mathrm{r}}^{\text {fast }}=$ $3.5 \mathrm{~ns}$ measured with different detectors and heavy ions as signal sources. The change sensitive slow part gives a negligible noise contribution to heavy ion energy spectra. Maximum length of the input cable for minimum risetime and maximum $\mathrm{s} / \mathrm{n}$ ratio is about $7 \mathrm{~cm}$. The preamplifier can be used in vacuum if kept at constant temperature below $25^{\circ} \mathrm{C}$. Main amplifier: bandwidth from 0 to $300 \mathrm{MHz}$ ( $D C$ to $\tau_{r}=1.2 \mathrm{~ns}$ ), 1 ow nnise, adjustable offsct, and excellent oulpul stability.

The achievable time resolution for the setup shown in Fig. 3.4-1 depends mainly on the type and quality of the detectors used and on their appropriate cooling and overbiasing, provided that all modules work rogularly and are adjusled properly. Figure 3.4-2 demonstrates the time resolution achieved with a surface barrier detector ( $\mathrm{dE} / \mathrm{dx}, \mathrm{E})$-telescope and 160 ions.

The system has been used successfully for the charge and mass identification of products from the reactions ${ }^{12} \mathrm{C}+14 \mathrm{~N}$ (see Sec. 14.1 of this report) and ${ }^{59} \mathrm{Co}+{ }^{35} \mathrm{Cl}$ (see Sec. 13.4 of this report) measuring specific energy loss, energy and time of flight. Figure $3.4-3$ gives a mass vs energy plot taken for products from the reaction $12 \mathrm{C}+{ }^{14} \mathrm{~N}$ at $\mathrm{E}_{\mathrm{lab}}=53.5 \mathrm{MeV}$. 
The mass resolution $\frac{\Delta \mathrm{m}}{\mathrm{m}} \propto \frac{\Delta t}{t}$ of a time-of-flight telescope cannot be improved without limit by enlarging the flight path and hence $t$. In order to achieve a reasonably large angular acceptance of the telescope and to keep losses due to multiple scattering from the start detector small, the stop detector must be sufficiently large. Large detectors, though, give worse time resolution $\Delta t$. Thus it is necessary to strive for good $\Delta t$ concurrently. With start detectors of $5 \mu$ and $10 \mu$ thickness and stop detectors of $100-300 \mu$ thickness and $450 \mathrm{~mm}^{2}$ area we achieved a typical time resolution of $160 \mathrm{ps.} \mathrm{Using} \mathrm{flight} \mathrm{path} \mathrm{lengths} \mathrm{of}$ $30-50 \mathrm{~cm}$ this gives a satisfactory mass resolution with an efficiency of $95 \%$ in the range $A \leq 20$ and at energies around $50 \mathrm{MeV}$, and with an efficiency between $90 \%$ and $10 \%$ in the range $20 \leq \mathrm{A} \leq 60$, depending on mass and energy (40-120 MeV) of the particle.

\subsection{Development of Electron Detector Telescopes}

\section{E.G. Adelberger and P.A. Dickey}

Development was begun late this year on a plastic scintillator telescope for use in the study of nuclear beta decay. The preliminary design for such an electron detector is illustrated in Fig. 3.5-1. The telescope consists of three elements -- a thin (.05") plastic $\Delta E$ detector, an active collimator (A), and an $E$ detector. Light guides are used to couple the $\Delta \mathrm{E}$ and $\mathrm{A}$ scintillators to photomultiplier tubes. The plastic elements were machined from Nuclear Enterprises NE 102 plastic-scintillator stock and optically polished. Aluminum foil and thin layers of aluminum evaporated directly onto the scintillators provide optical isolation between the active elements and act as reflectors to maximize the light output.

The electronics shown in Fig. 3.51 pertorm several vital functions. Valid electron events are defined by $\Delta \mathrm{E} \cdot \mathrm{E} \cdot \overline{\mathrm{A}}$ logic. The collimator defines the aperture of the telescope and vetoes events in which electrons backscatter from the $E$ detector. The fast coincidence between the $E$ and $\Delta E$ detectors discriminates against gamma rays because of their small probability of interaction in the thin $\Delta E$ plastic.

Tests on a prototypé telescope with conversion electrons and gamma rays from a $207 \mathrm{Bi}$ source promise a gamma ray rejection cxceeding $99 \%$, i.e., the efficiency for gamma ray detection is less than $1 \%$ of that for electron detection at the same energy. We are presently working to improve the energy resolution and extend the low energy threshold of

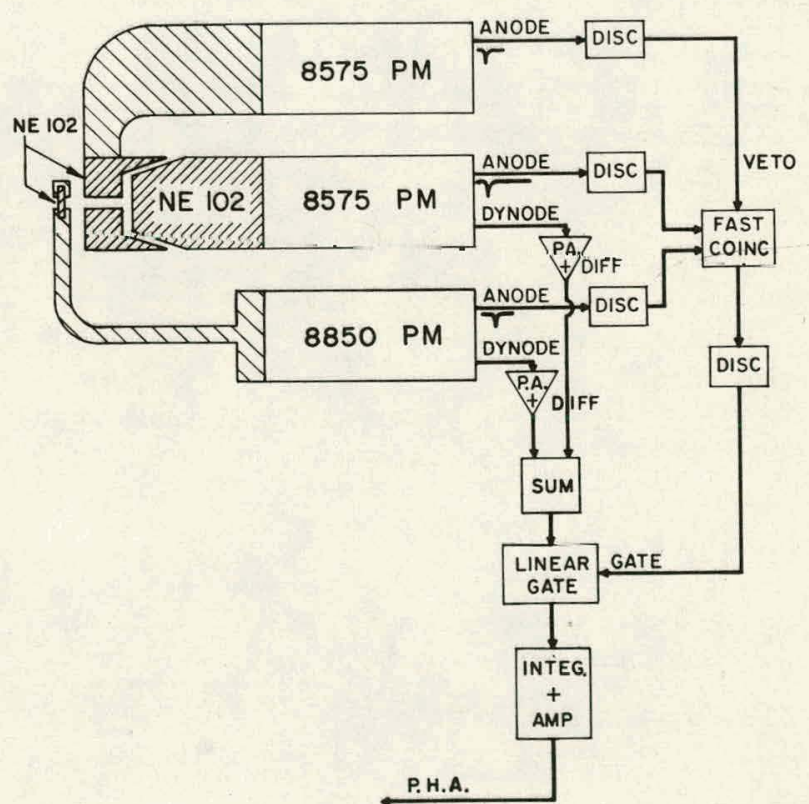

Fig. 3.5-1. Schematic diagram of electron detector telescope showing the arrangement of scintillators and typical electronics. 
the telescope.

An immediate application of this instrument is the measurement of the isospin forbidden beta decay branch of ${ }^{28} \mathrm{Mg}$ to the $\mathrm{O}^{+} \mathrm{T}=1$ level of $28 \mathrm{Al}$. The telescope is particularly well suited for this experiment because good $\gamma$ ray rejection is necessary to suppress the background of $\gamma$ rays from excited states populated by the isospin allowed beta decay channels.

\subsection{Construction of Scattering Chambers for Particle-Gamma and Gamma-Gamma Coincidence Experiments}

\section{H. Bohn, Y-d Chan, and B.A. Scott}

Two small T-shaped scattering chambers have been constructed for application in particle-gamma and gamma-gammd coincidence experiments. A general view of both scattering chambers is presenter in Fig. 3.0-1.

The hndy of both scattering chambers is made out of brass which allowed

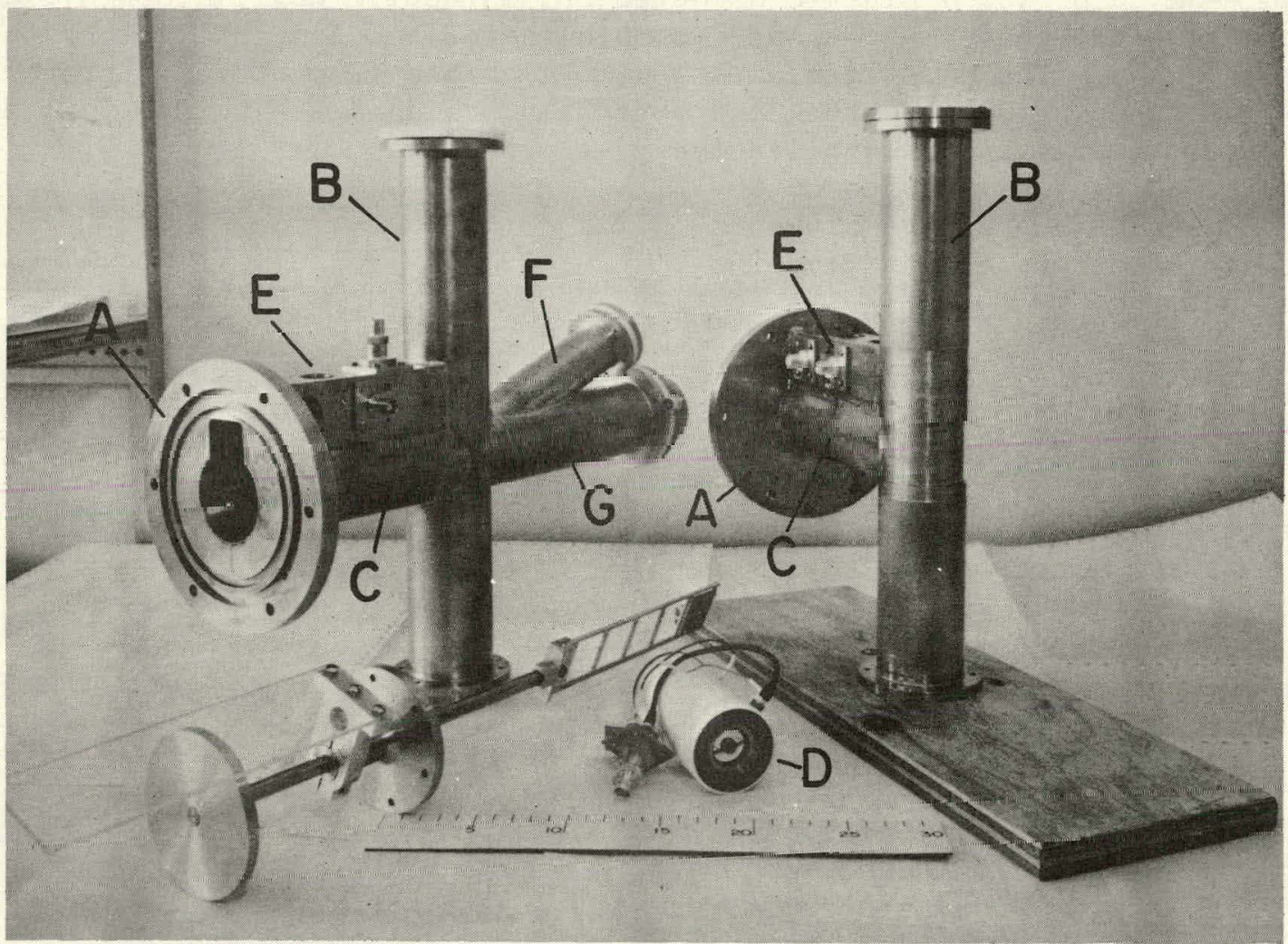

Fig. 3.6-1. General view of both scattering chambers. (A) standard flange; (B) vertical tube which contains the taryet ladder; (C) horizontal part which accepts the insert; (D) insert which contains the annulas detector and the beam collimation system; (E) rectangular top together with the BNC connectors; (F) side tube for housing the monitor detector; (G) Faraday cup extension. 
for easier fabrication compared with stainless steel. The chambers can be connected to the existing beam line system via a standard flange. The diameter of the inner bore for both the horizontal and vertical tubes is $5 \mathrm{~cm}$. This means that the distance from the target to the entrance window of a Ge(Li)-detector mounted outside of the scattering chamber can be as low as $26 \mathrm{~mm}$ with a chamber wall thickness of $41 \mathrm{~mm}$ in between.

The vertical tube part carries a standard target ladder ( 5 or 6 positions) which is electrically isolated from the rest of the chamber body and which can be fixed at any height and angle by a screw. The horizontal part which is placed between the target holding tube and the flange mentioned above was much harder to construct because it has to accept an insert made of teflon which in turn contains at the present time a special annular detector for the detection of heavy ions and a beam collimation system consisting of two apertures. The ring detector has a $6 \mathrm{~mm}$ hole and is carefully shielded from the beam by a thinwalled $\mathrm{Ta}$ tube which in turn is fixed in position by a Ta-aperture which covers the back side of the transmission type annular detector. The small Ta-tube actually consists of two parts which are held together with a "light push fit". The inner diameter is then $4 \mathrm{~mm}$. It is therefore possible to shield also the inhomogeneous part of the detector surface near the inner hole from particles back-scattered from the target. The relative positions of the apertures and the ring detector within the insert can be varied via different distance pieces. The whole insert can be moved within the horizontal brass tube and fixed at any position. For example, in order to measure low nuclear reaction cross sections the insert could be moved so that the distance of the surface of the annular detector to the target is as small as $8 \mathrm{~mm}$.

Because the annular detector is a transmission mount type detector the horizontal tube cross section is not a circle but rather is topped with a rectangular frame. Imbedded in this frame are the vacuum feedthroughs for the particle detector preamplifier connection and the aperture current readings. Two BNC-to-Microdot connectors are provided which would allow also the use of a $\Delta E-E$ annular detector telescope system. The inside of the rectangular top stores the cables to the detector and apertures. This method does not conflict with moving the insert.

Both the target holding tuhe and the annular detector holding device are identical for the two scattering chambers. They differ only in design after the beam has passed the target. Then one of the two chambers contains a Faraday cup extension together with an additional target centered side tube in which for example a monitor counter could be mounted. The Faraday cup is electrically isolated from the rest of the scattering chamber body and is designed to contain an inner tube (for example made out of lead) which could be extended in length very close to the target. This scattering chamber could be used for studying nuclear reactions with thin targets. The Ge(Li) detector is then closest to the target at $90^{\circ}$.

In the other scattering chamber the beam should be stopped by the target or a suitable backing, and the $\mathrm{Ge}(\mathrm{Li})$ detector could be moved between $90^{\circ}$ and $-90^{\circ}$ with respect to the beam axis with a constant wall thickness of $4 \mathrm{~mm}$ between target and $\mathrm{Ge}(\mathrm{I}, \mathrm{i})$. 


\subsection{Feedback of Spin Direction of the Polarized Beam}

E.G. Adelberger and H.E. Swanson

A new measurement of the parity violating asymmetry in the ground state doublet of ${ }^{19} \mathrm{~F}$ is planned later this year to improve the statistical and systematic accuracy of the result. It was found in the previous measurement ${ }^{1,2}$ that a significant though correctable instrumental asymmetry arises if there exists a component of polarization normal to plane of the $\gamma$-counters which switches sign with polarization state. When the source was operated in the mode used in the previous measurement ${ }^{3}$ the beam polarization was observed to wander by about 1 degree requiring occasional rotations of $\sigma_{p}$ about the beam axis to reset the vertical polarization component to zero. To eliminate these corrections to the data, it was decided to measure continuously the vertical polarization component and use it as an error signal; the polarized ion source's Wien filter is then rotated to minimize the error signal.

A polarimeter has been designed and constructed which will measure both the vertical and horizontal components "on line". It is an integral addition to the parity experiment gas cell described in References 1, 2 and 3. Figure 3.7-1 shows the polarimeter in cross section and its relationship to the gas cell. It consists of 4 ORTEC surface barrier detectors spaced $90^{\circ}$ apart azimuthally with

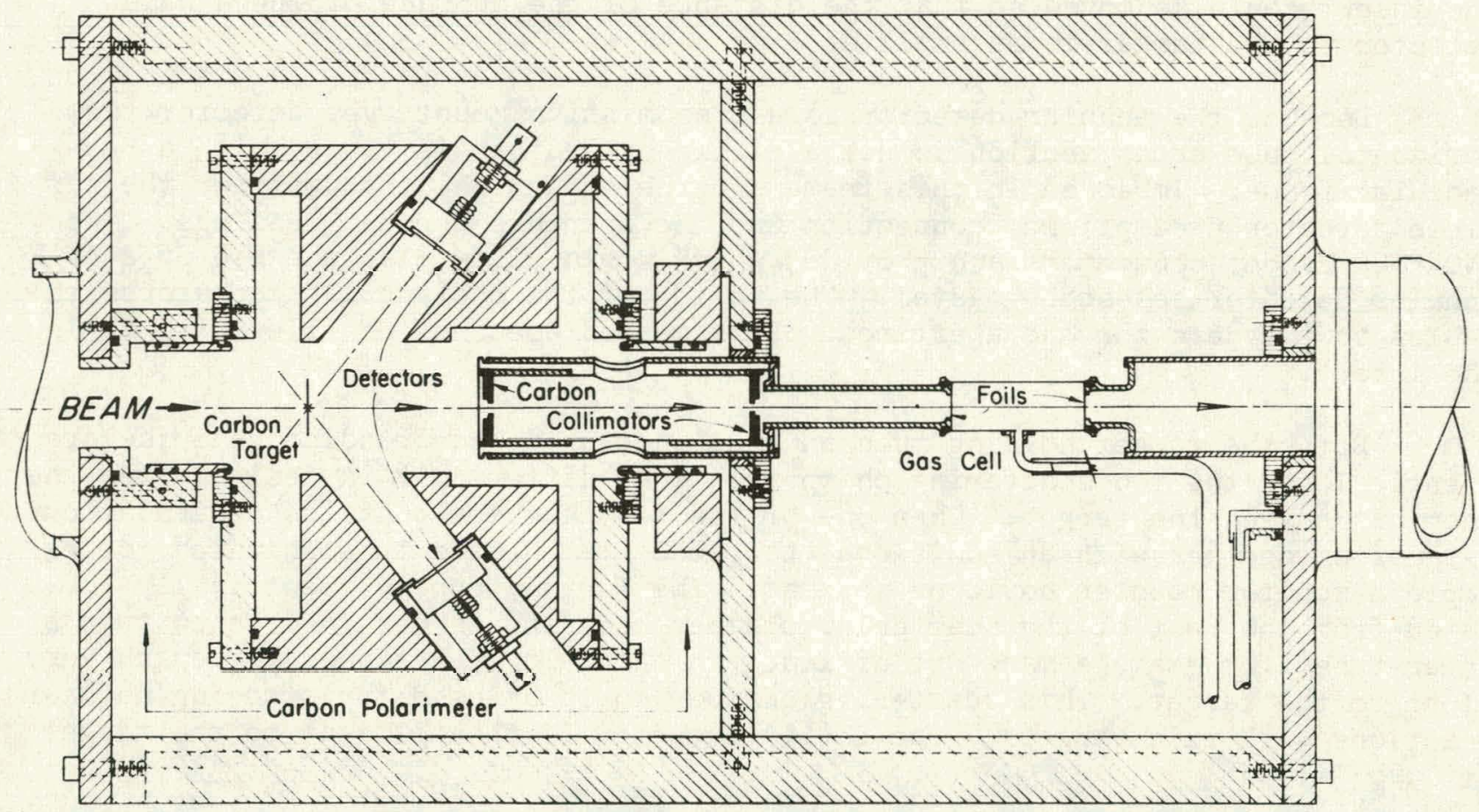

Fig. 3.7-1. Cross sectional view of polarimeter ${ }^{22} \mathrm{Ne}$ gas cell used in the ${ }^{19} \mathrm{~F}$ parity mixing experiment. The polarimeter is used to measure both the horizontal and vertical components of the beam polarization and constitutes the error signal of a closed loop digital feedback system. 
two in the horizontal and two in the vertical plane. Only the vertical pair is shown for clarity. Carbon was chosen as an analyzer because it has an adequate analyzing power $\left(-0.8\right.$ at $\left.55^{\circ} \theta_{\mathrm{L}}\right)$ at our energies $(4.93 \mathrm{MeV})$ and unlike a ${ }^{4} \mathrm{He}$ gas target, allows the counters to see the entire interaction region. Provisions have been made to align the horizontal pair using the same reference level as is used in aligning the $\gamma$-counters. Rotating vacuum seals allow the body of the polarimeter to rotate around the beam axis to achieve alignment. The distance between the carbon foil and the gas cell collimators was purposely kept as short as possible to prevent the beam multiple scattered in the foil from spreading sufficiently to hit the ${ }^{22} \mathrm{Ne}$ gas cell collimators. This maintains good transmission and a low $\gamma$-background.

In operation, scalers routed as a function of source polarization state will count pulses from SCA's placed around the elastic carbon peaks. The computer will read the scalers at each pass around the data collection wait loop and compute the direction and magnitude of the beam polarization. Statistically significant deviations of the spin direction from horizontal are converted into the number of pulses required by the Wien filter stepping motor to rotate the spin back to horizontal. The correction signal is displayed in an external buffer register.

1. Nuclear Physics Laboratory Annual Report, University of Washington (1974), P. 52 .

2. E.G. Adelberger, H.E. Swanson, M.D. Cooper, J.W. Tape, and T.A. Trainor, Phys. Rev. Lett. 34, 402 (1975).

3. H. E. Swanson, Ph.D Thesis, University of Washington, 1975, unpublished. 


\section{THE COMPUTER AND COMPUTING}

4.1 Computer System Improvements

\section{N.R. Cheney}

Twelve ADC's have been purchased by the Nuclear Physics Laboratory to replace the 10 year old units now in use with the on-line computer system. The instrument selected, Tracor Northerm model TN1213, has undergone considerable testing by the Laboratory to verify its performance. Several problems were discovered and are now being corrected by the manufacturer.

An interface for these units has been designed and is under construction. Some of its characteristics include: expandability to $16 \mathrm{ADC}$ 's; direct memory accese or software putaway of singles data; hardware or program formatting of coincidence data; mode and function control by program or by experimenter manipulated switches; simultaneous prosessing of silyles and coincidence events; 2 levels of event stacking; and 4 independent routers. Checkout of this hardware is expected to begin shortly.

\subsection{Revision of the CALCOMP Plotting System}

\section{K. Green}

The Laboratory's CALCOMP system programs have recently been rewritten in order to: (1) Correct errors, (2) optimize pen motion, (3) expand system capabilities, and (4) reduce storage requirements.

SYMBOL, the character output routine, can no longer accidentally displace a plot origin. It will now shift directly between superscript and subscript modes without first restoring to normal mode. Rotations are performed by TNS, ITNS, and ITNSXY, replacing several sections of code which operater onratically. Error bars now rotatc with lie plotting symbols.

DRVR, which transmits commands to the plotter, automatically disconnects the data channel after the transmission is complete, eliminating computer hangups. DRVR will now return a value indicating the current state of the pon, + for up and - for down.

NUMBER now goes directly to the position of the first digit. Leading blank chalacters have been suppressed.

Two new systerl routines, AXIS and NUMAX, perform the drawing and calibration of plot axes. AXIS is capable of drawing either a linear or logarithmic axis, starting from either end. There is a provision for starting an axis part way into a cycle. NUMAX will calibrate axes in either fixed point or powers of 10, again starting from either end of the axis. In addition, the numbers may be left, right, or center adjusted, and rotated to $0,90,1.80$, or 270 degrees.

MOVER, which was modified last year to permit the drawing of dashed or broken lines, has been fully incorporated into the system. An enable/disable 
feature has been added, so that the dashed lines may be turned off and on as desired. The system has been altered so that dropping the pen automatically enables dashed lines, and raising the pen disables. All symbol drawing routines and AXIS disable the dashed lines; they are unaffected by this option.

The Pankiewitz routines, a set of 5 general data plotting programs, have been rewritten entirely. They are now capable of semilog and full logarithmic plotting. One selects the log option by simply negating the length of the relevant axis. The routines will then correctly draw, calibrate, and plot, treating the axis as logarithmic.

These routines are compatible with the dashed line option. One can draw a dashed line simply by setting it up with the appropriate call to MOVER beforehand.

A new system writeup, detailing its use, is now available.

\subsection{Graphics on the Computer 2 Display}

\section{K. Green}

The computer 2 CRT display has been operating for a year now without an adequate graphics package. In order to alleviate this problem, an interface has been. written which transfers Calcomp output to the display. All of the Calcomp software is usable on the display.

The display is activated by the presence of two subroutines in the plot program, SETBF and ZAP. The appearance of either of these routines automatically forces the loading of a display version of DRVR.

SETBF has two purposes. It clears the display buffer and re-initializes for a new plot. It also, on its first call, loads the display program DISP onto the disc storage unit, and then converts the storage allocated to DISP into $a$ disc buffer. Display points go into this buffer as they are formed, and then onto the disc when filled. Each point is displayed once as it enters the buffer in order to give a visual indication of the program's progress. SETBF must be called before using any Calcomp routine on the CRT.

ZAP may be called at any time to display the plot image. This call has several effects. Any remaining points in the buffer are written onto the disc. An image of the entire core follows this. The display overlay program DISP is then loaded from the disc into low core and executed. DISP reads the disc for display points, loads them into core and then displays them, one point at a time, as quickly as possible.

Displays of more than $8 \mathrm{~K}$ points tend to flicker noticeably. To help reduce this, every other point is discarded before storage on the disc. The display resolution suffers, but not seriously.

The display continues until sense switch 4 is set. DISP then reloads the core image from the disc. Execution resumes normally from this point. 
This is an excellent way to debug plot programs. It is much faster and less wasteful of paper.

SETBF and ZAP are also described in the new Calcomp system description.

4.4 HOP-TWO: Version 4.1 for Iterated Optical Model Calculations

John G. Cramer

HOT-TWO is a heavy ion optical model program in wide use in the Laboratory, which is very powerful in its capabilities ( 500 partial waves, 600 radial integration steps, complicated potential forms, many options) but which is compact enough to operate on the Laboratory's SDS 930 computers. 1 One of the prices of operating on a small computer has been that the array storing the experimental cross section data to be compared with the calculations is overlaid during the calculation so that this data must be read in fresh for each calculation.

A new version of HOP-TWO (Version 4.1) has been written employing several space saving innovations, which eliminates this overlay of the input cross section data and provides a convenient means of varying any input parameter in a stepwise fashion. The "notch-perturbation" calculations described in Sec. 12.3 of this report were done using this program, simply varying the notch position as one of the parameters of the optical model potential.

1. Nuclear Physics Laboratory Annual Report, University of Washington (1975), p. 45; (1974), p. 26 .

4.5 COULIT, A Compact Coulomb-Nuclear Inelastic Scattering DWBA Program

John G. Cramer and C.K. Gelbke ${ }^{\dagger}$

In Sec, 12.? nf thio repurl we discuss some recent inelastic scattering calculations in which the Austern-Blair approximation is compared with more accurate DWBA calculations in the Coulomb-nuclear interference region. To perform these calculations, it was necessary to have a working Coulomb-nuclear DWBA code with enough partial waves for heavy ions into which the Austern-Blair radial integrals could be substituted. We developed such a program, first on the Laboratory SDS 930 computer and later on the Heidelberg CDC 3600 computer system. The result is COULIT, a very compact DWBA program which is capable of performing these complicated calculations.

Our starting point was a light ion DWBA program written at this Laboratory by W.J. Braithwaite. 1 This was a compact DWBA code, but it lack capabilities for calculating Coulomb excitation, and it was too restricted in the number of partial. waves available. The program was simplified and restructured for more array space and provision was made for the large radial integration needed for the Coulomb excitation calculation of the lower partial waves. A difficulty encountered in the latter was that generation of the waves directly from the Schrödinger equation over the large radial intervals required resulted in 
cumulative errors which destroyed the accuracy of the calculations. This problem was solved by doing the Coulomb radial. integrals in segments. At the beginning of each segment new Coulomb functions are determined and, along with the previously calculated elastic scattering amplitudes, used to obtain the starting values for the Schrödinger equation solutions. Thus cumulative phase errors in the Coulomb waves are not transmitted from one segment to another and the procedure gives reliable coulomb excitation integrals.

For the higher partial waves, where the nuclear potential and its distorting effects can be neglected, we used the Coulomb excitation program of Samuel and Smilansky ${ }^{2}$ which employs very fast and stable recursion relations to calculate the Coulomb radial integrals.

The program also contains many options for substituting the various forms of the Austern-Blair approximation for the more accurate DWBA calculation so that comparisons can be made. In the light of the success of these comparisons, it is clear that a very fast and accurate Austern-Blair Coulomb-nuclear code could be written by making a cut-down version of COULIT, and we are contemplating this step.

The results of COULIT DWBA calculations were compared with calculations done with DWUCK and with the original Braithwaite program, and found to be in excellent agreement. We feel that compactness and flexibility of this program gives it many advantages over larger codes for investigations such as those we have completed.

+ Summer visitor, 1975; present address: Lawrence Berkeley Laboratory, Berkeley, CA 94720 .

1. Wilfred J. Braithwaite, private communication.

2. M. Samuel and U. Smilansky, Comp. Phys. Comm. 2, 455 (1971).

4.6 GELIFIT: A Peak and Background Fitting Program for Ge(Li) $\gamma$-Ray Entrgy Spectra

H. Bohn and Y-d Chan

A peakshape fitting and spectra analyzing program päckage GLLIFIT has heen written to run on the Nuclear Physics Laboratory off-line No. 2 computer. This program has the following special features:

a) It makes extensive use of the newest computer 2 peripheral devices including the disc storage and the display unit. Using the disc unit speeds up the input-output process considerably and also in effect reduces the maintenance rate on the Lineprinter and Magnetic Tape Drives.

b) It has very flexible peakshape functional forms that are particularly. relevant for spectra from $\mathrm{Ge}(\mathrm{Li})$ detectors. The fitting spectrum is composed of two parts. The first part is a general background represented by a polynomial function. The second part is a sum of several peaks, with each peak being composed of the following:l 
(i) A normal symmetric Gaussian plus complimentary error function folded exponential tails on both low- and high-energy sides of the peak;

(ii) A step function background which is also folded with a complimentary error function under the peak area.

A total of 9 independent parameters are needed for each peak. Any of the parameters can be either held fixed or independently varied.

Details of the definition of the parameters and other programming information can be obtained from the NPL Program Library.

c) The program execution can be interrupted any time with intermediate results displayed on the CRT unit. Traces of different intensitios dioplay correspondingly the data points, the best and the next best fitting curve so far, the background curve, and the fitted peaks in both linear and log scalc simultaneousily.

The setup of the program is such that only a minimal input is required from the user. Initial parameters are deduced from the input information and then the fitting starts. An optional memory switch can be set so that the geometrical form factor of the fitting region will be carried over to the next fit, only with the overall intensity readjusted. In actual running tests, this proves to drastically speed up the job execution time.

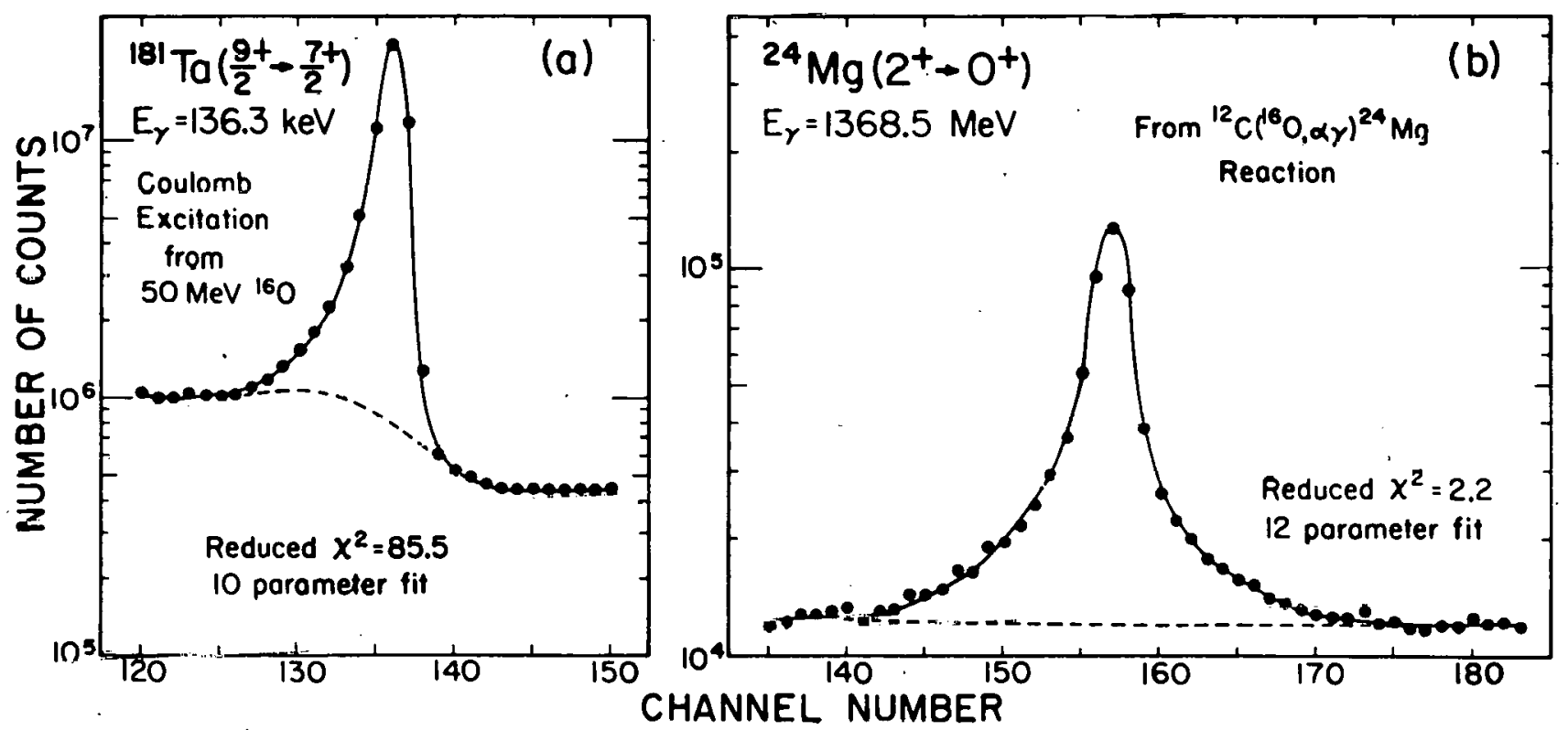

Fig. 4.6-1. Example of fitting results.. The circles indicate the experimental data. The dashed line indicates the calculated background level and the solid line is the calculated fitting curve. An extra exponential tail on the high energy end was added in the fitting of (b). 
As illustration, the fitting results of two peaks with very different shapes are shown in Fig. 4.6-1. Further information and limitations of the code can be obtained from NPL Program Library Instruction.

1. The parameterization we used is very similar to that used by G.W. Phillips and K.W. Marlow, NRL Memorandum Report 3198, Jan. 1976, except that we allow also a high energy exponential tail. 


\section{ATOMIC PHYSICS}

5.1 The Question of Electron Pickup During the $\alpha$ Decay of ${ }^{210}$ Po

D. Burch, P. Dyer, H.J. Fischbeck ${ }^{\dagger}$, and M.S. Freedman ${ }^{\dagger+}$

We have completed our work on an attempt to verify the proposal that Lshell vacancy production during $\alpha$ decay of 210 Po occurs primarily by electron pickup to a bound state of $\mathrm{He}^{+}$. The experiment and results are described in more detail elsewhere. 2 The Po source was prepared in the NPL hot lab by autodeposition onto $\mathrm{Ag}$ from a micropore-filtered solution of Po prepared at Argonne National Laboratory. Apparatus for detecting $\mathrm{He}^{+}$ions in coincidence with $\mathrm{L} x$ rays cmitted from a thin Po source is shown schematically in Fig. 5.1-1, and the singles and coincidence particle opectro accumulated in 137 hours are presented in Fig. 5.1-2. The number of $\mathrm{He}^{+}$inns

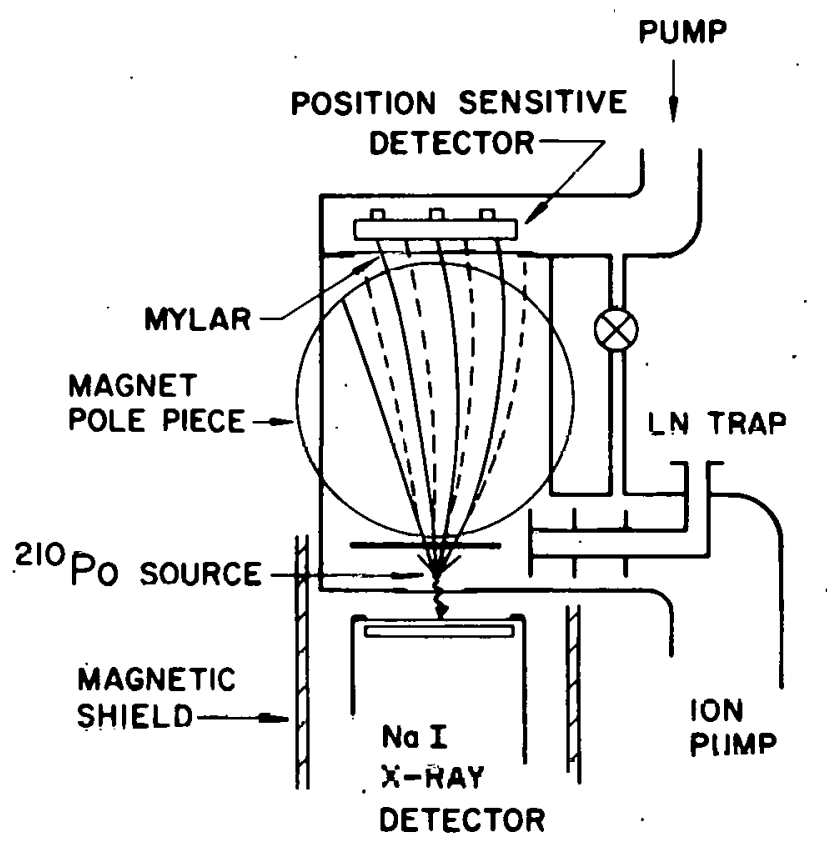

Fig. 5.1-1. Solieilutic diagrall of the apparatus. The paths of the $\alpha$ particles (solid lines) and the $\mathrm{He}^{+}$ions (dashed lines) as separated in the magnetic field following a multi-slit collimator are shown schematically. The drawing is roughly to scale with the pole piece diameter equal to $10 \mathrm{~cm}$.

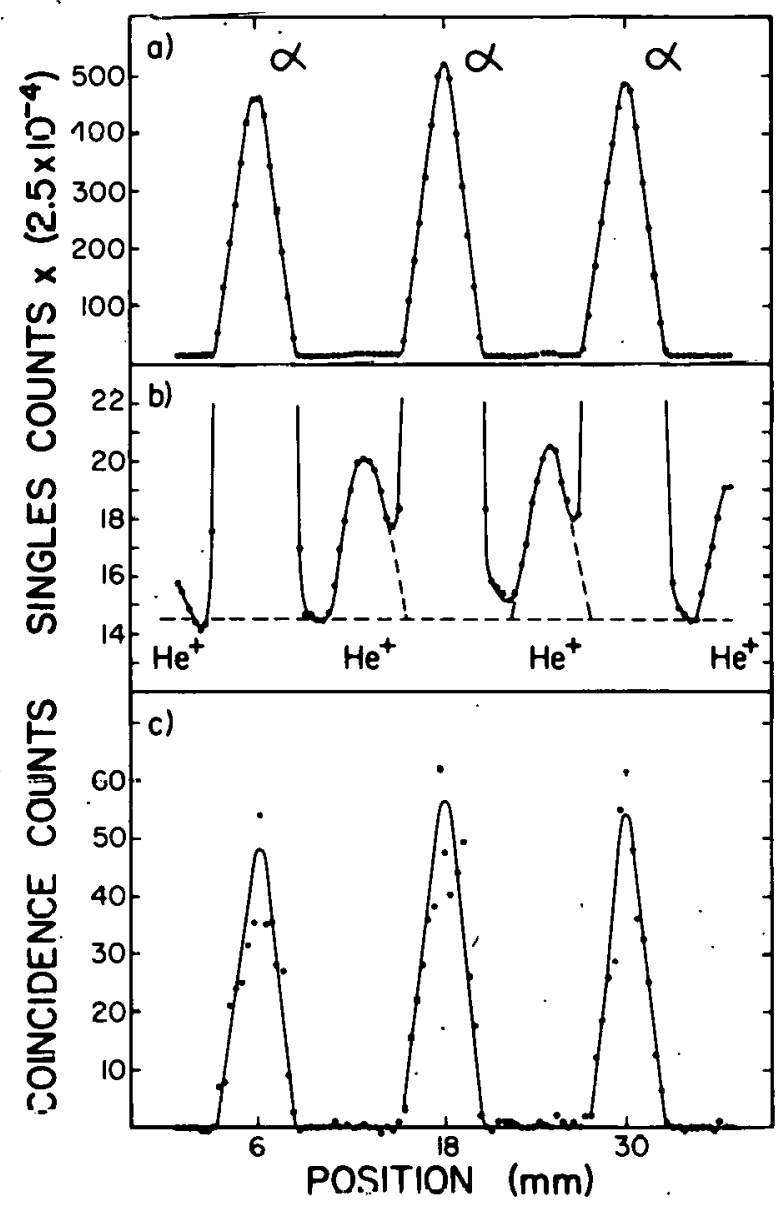

Fig. 5.1-2. Position sensitive detector spectra. (a) Singles spectrum of all particles with no coincidence requirements, (b) the same spectrum expanded and and offset to show the $\mathrm{He}^{+}$components, and (c) the spectrum of true coincidences with $\mathrm{L}$-rays. 
in coincidence with $L \times$ rays relative to the number of $\alpha$ particles was found to be

$$
\left(\mathrm{He}^{+} / \alpha\right)_{\mathrm{L}}=\left(0.7_{-0.7}^{+0.3}\right) \%
$$

The consequences of this result can, unfortunately, only be considered in terms of several assumptions. We clearly do not observe the ratio of $65 \%$ predicted by the earlier $\alpha$ and electron-spectroscopic data. ${ }^{1}$ However, the Po sources were not prepared in the same manner. The influence of this difference is uncertain, but is perhaps critical. Since the He ${ }^{+}$electron-loss cross section is $22 \times 10^{-17} \mathrm{~cm}^{2}$, half of the $\mathrm{He}^{+}$electrons would be lost traversing the equivalent of $\sim 25$ monolayers of material. We have no reason to believe that the surface contamination of the autodeposition sources used for a period of six days should be any greater than that of the retarded-beam sourcesl used for a period of sixty days in a spectrometer chamber pressure 100 times higher. Furthermore, to maintain the electron-capture hypothesis we must assume that there was not significant surface contamination to the retarded-beam sources. Following this line of reasoning, then, the primary uncertainty in this comparison is the degree of Po agglomeration in the two types of sources. It is known that thin layers of radioactive materials collected by autodeposition do agglomerate on the collector surface to a high degree. On the other hand, the microscopic behavior of a retardedbeam deposition of Po has not been studied. The crucial question is whether beam deposition at $200 \mathrm{eV}$ onto both $\mathrm{C}$ and $\mathrm{Al}$ could each be significantly different from autodeposition onto $\mathrm{Ag}$ with regard to agglomeration.

If we assume that the microscopic structures resulting from the two modes of source preparation are comparable, then this experiment rules out the electroncapture explanation of the discrepancies found in the earlier coincidence measurements.

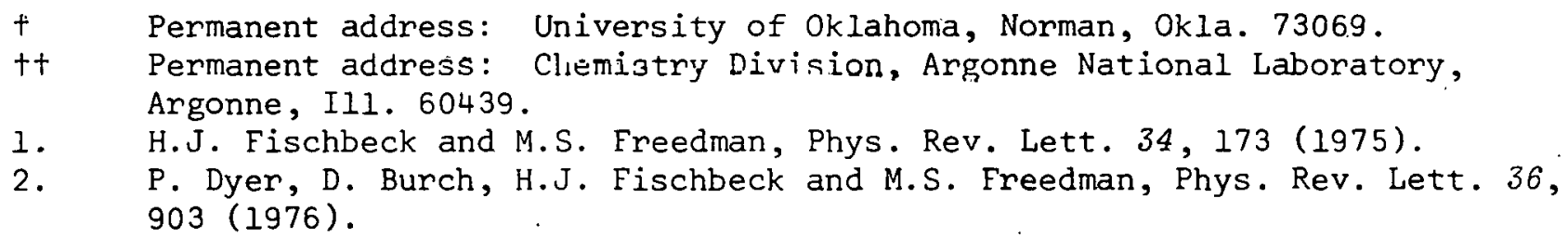

\subsection{Cross Sections for K-Shell Ionization by Electron Impact}

D. Burch and K. Green

Experimental values' of total cross sections for $\mathrm{K}$-shell ionization by incident electrons $\left(\sigma_{K}\right)$ have been compiled from the literature for $H$ through $B i$ for electron energies ( $\mathrm{T}_{e}$ ) from threshold up to $900 \mathrm{MeV}$. A semi-empirical formula was developed from weighted fits to the data which can be used to estimate the cross sections for targets and energy regions not covered in the experimental data. The compilation will be submitted to Atomic Data Tables; the format for the presentation is shown in Fig. 5.2-1. The semi-empirical formula is

$$
\sigma_{k} / \sigma_{0}=\left(\frac{16 b}{4+\ln k}\right) \frac{\alpha^{2} u}{\beta^{2}}\left[\ln \left(\frac{\beta^{2}}{1-\beta^{2}}\right)-\beta^{2}+\ln \left(\beta_{0}^{-2} k^{1-\beta_{0}^{2} / \beta^{2}}\right)\right]
$$


where $\sigma_{0}=8 \pi a_{0}{ }^{2} / u^{2}$ with $u$ equal to the $\mathrm{K}$-shell binding energy in Rydberg units $\left(u=u_{k} / R\right), \alpha=2 R / m c^{2}=1 / 137$.

$$
\begin{aligned}
& \beta^{2}=1-\left(1+T_{e} / m c^{2}\right)^{-2}, \\
& \beta_{0}^{2}=1-\left(1+u_{k} / m c^{2}\right)^{-2}
\end{aligned}
$$

$b$ and $K$ are empirical parameters given in Fig. 5.2-2. We estimate that this formula should be accurate to $\sim 30 \%$ for any target and any electron energy in the range investigated.

The subject of $\mathrm{K}$-shell ionization by electrons has recently been ravicwcd by Powe 11. 1

1. C.J. Powell, Rev. Mod. Phys. 48, 33 (1976).

\subsection{Multiple Scattering of Heavy Ions}

D. Burch and K. Green

Small angle multiple scattering distributions have been measured for oxygen ions of 20 to $50 \mathrm{MeV}$ in thick targets of $\mathrm{Al}, \mathrm{Ni}$, and $\mathrm{Au}$ with energy losses in the foils of up to $80 \%$ of the incldent energy. The distuibutions are well reproduced by the classinal theory of 3 igmund and Winterbon ${ }^{1}$ by replacing the incident energy $E$ with the average energy in the foil $E-\Delta E / 2$. The theoretical scattering distributions are tabulated. 1 The half angles can be $c x-$ pressed as

$$
\alpha_{1 / 2}(\operatorname{deg})=1.10 \times 10^{-3} \frac{\mathrm{Z}_{1} \bar{Z}_{2}{ }^{2}}{E(\mathrm{MeV})} \tau^{\mathrm{N}} \text {, }
$$

where

$$
\tau=41.5 \frac{\mathrm{T}_{2}\left(\mu \mathrm{g} / \mathrm{cm}^{2}\right)}{\mathrm{z}^{2} \mathrm{M}_{2}(\mathrm{amu})}, \quad \mathrm{z}=\left(\mathrm{z}_{1}{ }^{2 / 3}+\mathrm{z}_{2}{ }^{2 / 3}\right)^{1 / 2} \text {, }
$$

and

$$
N=[\ln (1.03+\tau)]^{-0.115}-0.115 \text {. }
$$

The tabulated values of Meyer ${ }^{2}$ do not extend to the high $\tau$ values of the 
present data, but extrapolated values severely overestimate the half-angles.

We have compared Eq. (1) with heavy ion data at energies of several $\mathrm{keV}$ up to hundreds of $\mathrm{MeV}$, and in general it reproduces the experimental data to within $\pm 20 \%$. Our is the first data, however, to test this result for nonnegligible energy losses.

We have also shown that the earlier theories of Moliere ${ }^{3}$ and of Bohr and Williams ${ }^{4}$ will, with some modifications, yield a half-angle formula in a similar power-law form as Eq. (1). These modified theories are compared in Fig. 5.3-1.

1. P. Sigmund and K.B. Winterbon, Nucl. Instrum. Methods 119, 541 (1974).

2. L. Meyer, Phys. Stat. Sol. 44 , 253 (1971).

3. G. Moliere, Z. Naturforsch $3 a, 78$ (1948).

4. N. Bohr, Mat. Fys. Medd. Dan. Vid. Selsk 18, No. 8 (1948); E.J. Williams, Rev. Mod. Phys. 17, 217 (1945).

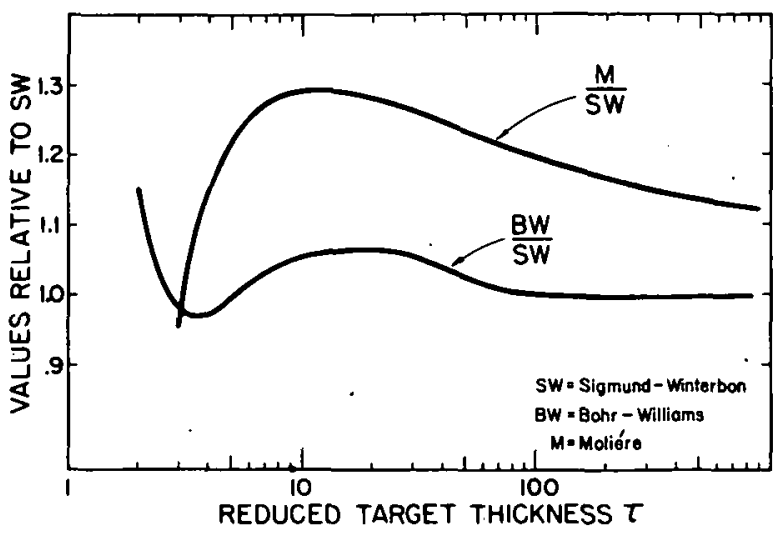

Fig. 5.3-1. Comparison of theoretical half-angles for multiple scattering of heavy ions. The results of Refs. 3 and 4 have been modified to improve the treatments of atomic screening. The Meyer results (Ref. 2) agree with Sigmund and Winterbon for $\tau<20$, but appear to be numerically unreliable at higher values.

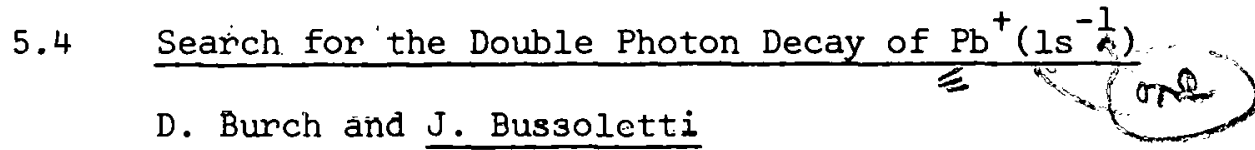

Our attempt ${ }^{l}$ to measure the ratio of double to single photon decay of $a \cdot k$ vacancy in $\mathrm{Pb}^{+}$was pursued with higher singles data acquisition $\left(5 \times 10^{9} \mathrm{~K} \times\right.$ rays) and a new, $90^{\circ}$ geometry. In this geometry the two detectors were separated by a $\mathrm{Pb}$ absorber' which completely eliminated Compton "cross talk" between detectors.. These improvements, however, did not significantly reduce our limit on this branch since we had, in fact, underestimated this limit in the earlier report due to errors in the efficiency calculations.

At present we can only conclude with confidence that

$$
\frac{\Gamma_{\gamma \gamma}}{\Gamma_{\gamma}} 6 \times 10^{-5}
$$

A theoretical treatment of this decay mode by Freund ${ }^{2}$ predicts (in our evaluation of $i t$ ) a branch for $\mathrm{Pb}^{+}$of slightly less than the hydrogenic value of $3 \times 10^{-5}$. We have recently learned, however, that this prediction should only be considered an order of magnitude estimate, and, further, that coherence effects could, in principle, increase the branch above the hydrogenic value. ${ }^{3}$ Our result shows that this increase, if present, is at least not greater than a factor of two. 
1. Nuclear Physics Laboratory Annual Report, University of Washington (1975), p. 191.

2. I. Freund, Phys. Rev. A 7, 1849 (1973).

3. I. Freund, private communication.

\subsection{K-Shell Ionization in $\alpha$ Decay vs $\alpha$ Collisions}

R. Bangert, D. Burch, and M. Roth ${ }^{\dagger}$

In 1965 Ciocchetti and Molinari ${ }^{1}$ proposed a method for nuclear lifetime measurement using the interference phenomena they predicted for atomic K-shell excitation during the approach and exit paths of the nuclear collision -- the phase difference being determined by the intermediate state lifetime. The technique might provide a means of studying very short lifetimes within a range of approximately an order of magnitude centered at a time $n / U_{K}$, where $U_{K}$ is the $K$ shell binding energy, i.e., (10-16 - 10=17)/Z2 sec. According to their results the lifetime could be determined by comparing calculated anisotropy coefficients $B$ with those extracted from the ionization probability at scattering angles $\theta>$ $90^{\circ}$ which is predicted to have the form

$$
P_{K}(\theta)=A(1+B \cos \theta) \text {. }
$$

More recently, Andersen et al. ${ }^{2}$ obtained a similar result from a different theoretical approach, and, further, experimentally observed the predicted anisotropy in 0.5 to $2-\mathrm{MeV} \mathrm{P}+\mathrm{Cu}$ collisions. Of particular interest to the present work is their prediction that this type of interference should result in an ionization probability during nuclear $\alpha$ decay which is $1 / 4$ of that for an equivalent energy a particle backscattered (zero impact parameter) from the daughter product.

We have investigated this proposal for the $\alpha$ decay of ${ }^{210}$ Po and find, instead, a ratio of $1 / 2$ which suggests that coherent interfcrence is nut occurring in the prodioted manul for the $\alpha$ collision. The same apparatus was used for the Po source and collision measurements. Our value of $P_{K}$ for $P o$ is compared with previous values in Fig. 5.5-1; the energy dependence of the collision data is shown in Fig. 5.5-2 compared with earlier data. The results agree with the semiclassical calculations except at the lowest energy where $15^{\circ}$ scattering, used in the available calculations, is no longer a good approximation to the near-zero impact parameters $\left(150^{\circ}\right.$ to $\left.174^{\circ}\right)$ of the experiment.

['ت1" Lhe $\alpha$ energy equivalent to that of the decay, the result is

$$
\frac{\mathrm{r}_{\mathrm{K}}(\alpha \text { collision })}{\mathrm{P}_{\mathrm{K}}}\left(\alpha \frac{\alpha \text { decay })}{1.96 \pm 0.17}\right. \text {. }
$$

On the basis of model calculations, we suspect that the predicted value of 4 for this ratio will only obtain in the limit of a velocities appruaching that of the $K$ clectruns. In the case of 210 Po the $\alpha$ velocity is much less than the average $\mathrm{Pb} K$ electron velocity. 


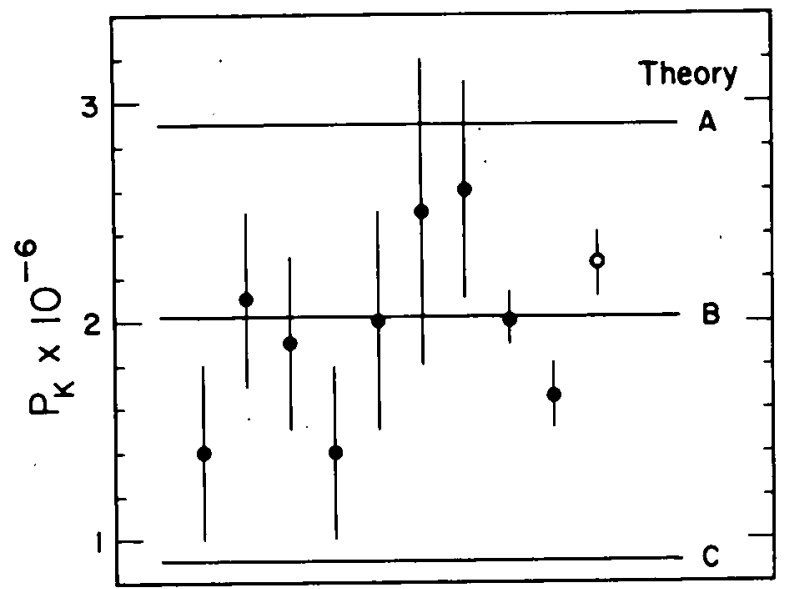

Fig. 5.5-1. Comparison of measurements and theoretical values for the $K$-shell ionization probability during the $\alpha$ decay of 210 Po. The open circle is the present value, earlier measurements are shown as solid points, and three theoretical values $(A, B$, and $C$ ) are shown as solid lines. The previous measurements and theoretical values are discussed in Ref. 3.

$+\quad$ Present address: Ruhr University, Bochum, Germany.

1. G. Ciocchetti and A. Molinari, Nuovo Cimento 40B, 69 (1965).

2. J.U. Andersen, E. Laegsgaard, M. Lund, C.D. Moak, and L. Kocbach, to be published in Phys. Rev. A.

3. H.J. Fischbeck and.M.S. Freedman, Phys. Rev. Lett. 34, 173 (1975).

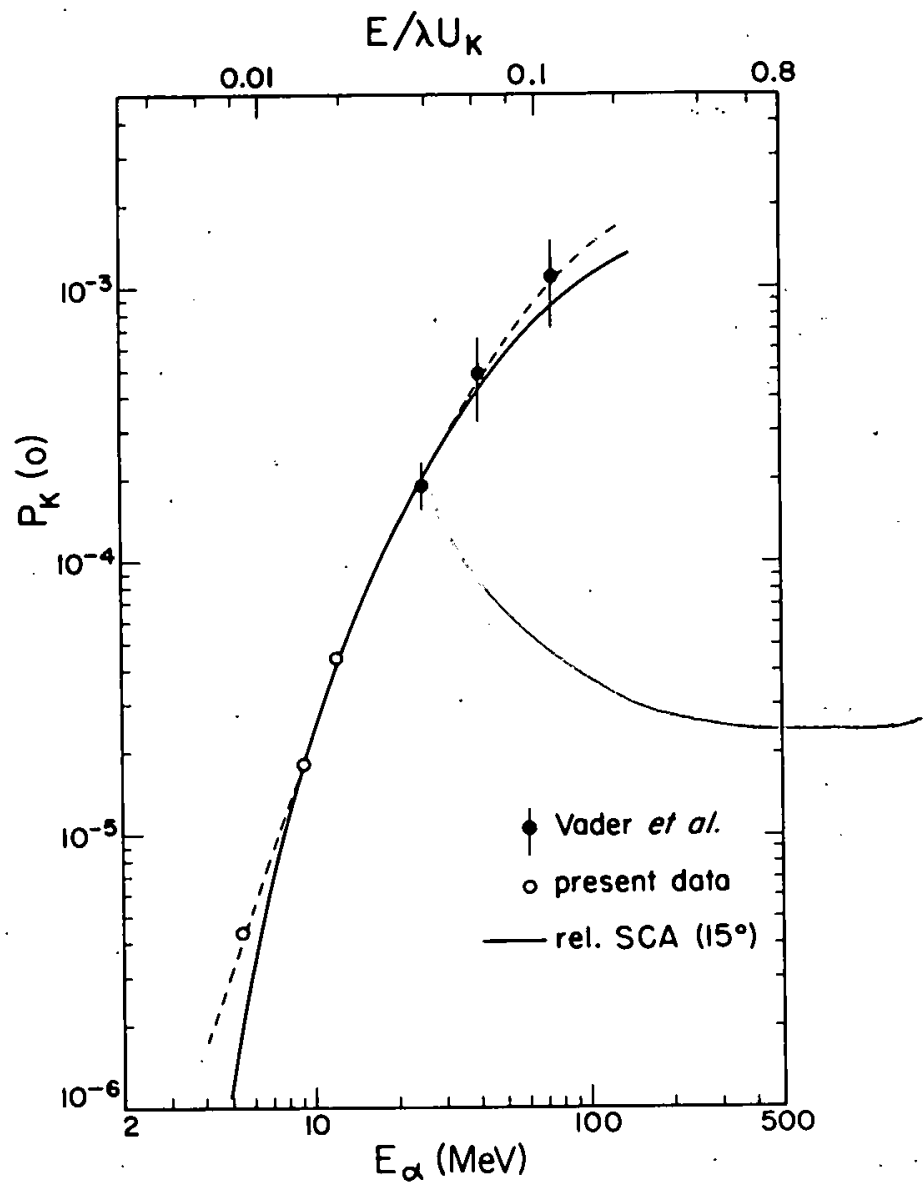

Fig. 5.5-2. Probability for K-shell ionization at zero impact parameter for $\alpha+\mathrm{Pb}$. Experimental uncertainties in the present data are $\pm 5 \%$. The data of vader etal.will be published in Phys. Rev. A.

5.6 Parameterization of Ionization Probabilities in Ion-Atom Collisions

D. Burch and K. Green

In an attempt to find systematic behavior in $\mathrm{K}$-shell ionization probabilities as a function of impact parameters, we have fit available data with several empirical functions. This effort has only been partially successtul. We find, for example, in fits of $\sim 30$ measurements on widely varying collision systems, that a Woods-Saxon shape will reproduce the probability distributions very well, but there appears to be no systematic behavior in the width parameter. This form can be used, purely empirically, for determining the total cross section from the integrated probability distribution. 
All distributions regardless of collision system fall off exponentially as $\exp (-b / a)$ for intermediate to large impact parameters $b$. The average value of $a$ for all systems is $a \sim R_{a} / 2=\hbar v / 2 U_{k}$, with variations extending from $R_{a} / 3$ to $\mathrm{R}_{\mathrm{a}}$.

A Gaussian shape is in general not a very good representation of the probability distributions. Fits on the basis of a modified Bessel function, as predicted by a simple model of the ionization, are now in progress:

A parameter $x$ defined by $\sigma=P(0) \pi x^{2}$ was extracted from the data to facilitate the prediction of probabilities at zero impact parameter. For the typical case, in which the distribution width is small compared to $a$, we find that $a \sim 1.3 \times$ and thus, as regards order of magnitude,

$$
\mathrm{P}_{\mathrm{K}}(0) \sim 21 / \mathrm{R}_{\mathrm{a}}^{2}
$$

for any collision studied so far. Considerably more accurate estimates can be made for specific ions.

\section{$5.7 \quad$ K-Shell Ionization of $\mathrm{Pb}$ by Heavy Ions}

D. Burch, P. Dyer, and K. Green

We have carried out the following measurements to investigate $K$-shell ionization of a high $\mathrm{Z}_{2}$ target by relatively high $\mathrm{Z}_{l}$ projectiles: The $\mathrm{Pb} \mathrm{K}-\mathrm{shell}$ ionization probability at zero impact parameter for $1.379 \mathrm{MeV} / \mathrm{amu} \mathrm{He}, \mathrm{Cl}$, and $\mathrm{Br}$ ions; the total cross sections, at this same velocity, for $\mathrm{H}, \mathrm{He}, \mathrm{C}, \mathrm{Cl}$, and $\mathrm{Br}$ ions; and the total cross section for 80 to $120 \mathrm{MeV} \mathrm{Br}$ inns. The data are nut yct analyzed in final form. A comparison of the first two data sets will show if the breakdown of $\mathrm{Z}_{1}{ }^{2}$ scaling is the same at zero impact parameter as in the total cross section -- an integral over all impact parameters. Combining the last set of data with our earlier meacurcments will yleld the variation in incident energy dependence with $z_{1} l$ at a fixed velucity.

1. See Sec. 5.5 of this report, and D. Burch, W.B. Ingalls, H. Wieman, and R. Vandenbosch, Phys. Rev. A 10, 1245 (1974). 


\section{NUCLEAR ASTROPHYSICS}

\subsection{Gamma-ray Production Cross Sections}

D. Bodansky, D. Chiang, and P. Dyer

We have begun a program to measure gamma-ray production cross sections to complement astrophysical observations of nuclear gamma-ray lines. Such lines have already been observed from solar flares, from the galactic center, and from Centaurus A. At such sites, energetic particles, primarily protons and alpha particles, collide with nuclei in the ambient medium (or, conversely, energetic heavy particles collide with $\mathrm{H}$ and He nuclei) to yield nuclear gamma rays via reactions such as ${ }^{12} \mathrm{C}\left(\mathrm{p}, \mathrm{p}^{\prime}\right)^{12} \mathrm{C}(4.44 \mathrm{MeV})$ and $160\left(\mathrm{p}, \mathrm{p}^{\prime}\right)^{16} \mathrm{O}(6.13 \mathrm{MeV})$. By observing these gamma rays, it is possible to obtain information on the energy spectrum of the interacting particles, the relative abundances of nuclei in the ambient medium, and the directions of the projectiles (through Doppler shift measurements). These investigations require accurate knowledge of nuclear cross sections and of gamma-ray lineshapes.

We plan to measure gamma-ray production cross sections over a range of bombarding energies for protons and alpha particles, incident on targets composed of the most abundant heavier isotopes in the universe such as $12 \mathrm{C}$, ${ }^{14} \mathrm{~N}, 160,20 \mathrm{Ne},{ }^{24} \mathrm{Mg}, 27 \mathrm{Al}, 28 \mathrm{Si}$, and $56 \mathrm{Fe}$. For the lighter nuclei, we will measure Doppler-shifted lineshapes as a function of the gamma-ray angle. In anticipation of the use of large, highresolution solid-state detectors in space, our measurements are being made with $\mathrm{Ge}(\mathrm{Li})$ detectors.

In an exploratory run, we bombarded thick $\mathrm{C}, \mathrm{SiO}_{2}$, and ${ }^{56} \mathrm{Fe}$ targets with 5 to $15-\mathrm{MeV}$ prutons and obeerver gamma rays at $90^{\circ}$ with a large $\mathrm{Ge}(\mathrm{Li})$ detector placed $20 \mathrm{~cm}$ from the target. Yields for production of $4.44-\mathrm{MeV}$ gamma rays from ${ }^{12} \mathrm{C}$ and a number of gamma rays from $160,{ }^{28} \mathrm{Si}$, and ${ }^{56} \mathrm{Fe}$ were measured. The yields from $\mathrm{p}+{ }^{56} \mathrm{Fe}$ are shown in Fig. 6.1-i. Gamma rays of $0.847,1.238$; and $1.811 \mathrm{MeV}$ come from the ${ }^{56} \mathrm{Fe}\left(\mathrm{p}, \mathrm{p}^{\prime}\right)^{56} \mathrm{Fe}$ reaction, while thuse of $0.812 \mathrm{MeV}$ come from ${ }^{56} \mathrm{Fe}(\mathrm{p}, \mathrm{n})^{56} \mathrm{Co}$. These ${ }^{56} \mathrm{Fe}$ cross sections are of particular interest, since this is a case in which two or more lines having different excitation functions are produced in the same

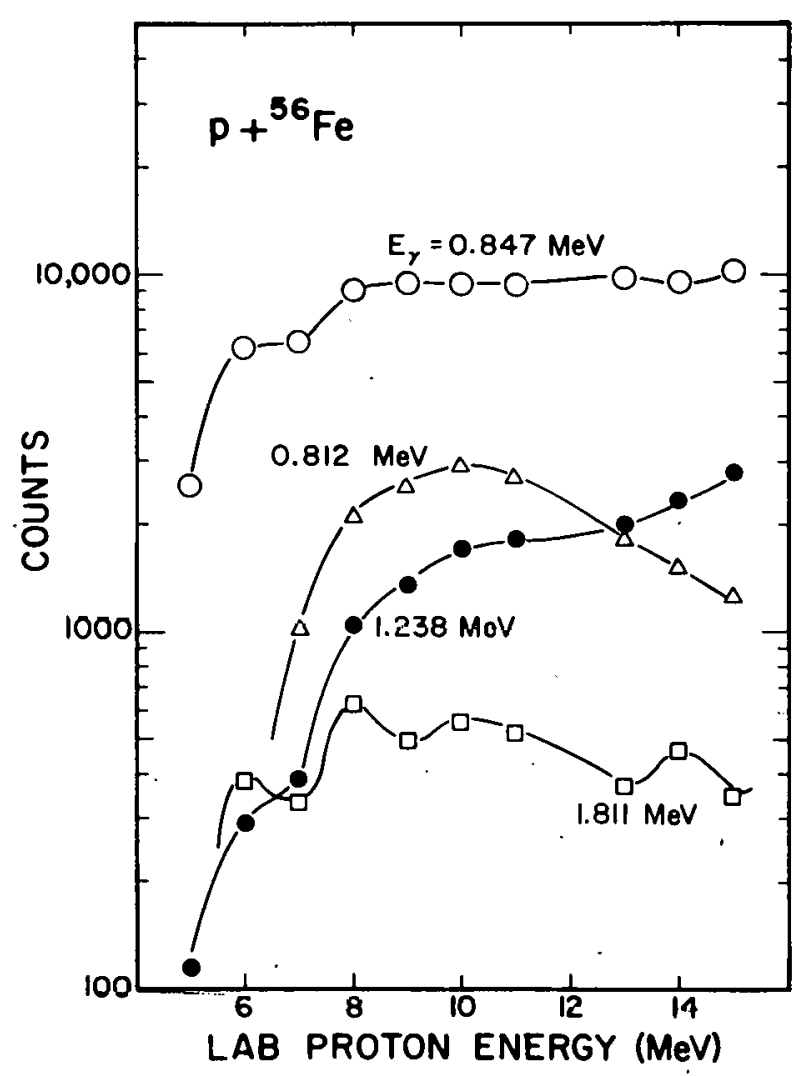

Fig. 6.1-1. Number of gamma rays detected at $90^{\circ}$ from the $\mathrm{p}+{ }^{56} \mathrm{Fe}$ reaction. 
target. The astrophysically observed ratio of two line intensities depends on the excitation functions and on the energy spectrum (but not on the absolute number) of the interacting particles. Since there are no highly-abundant isotopes heavier than $56 \mathrm{Fe}$, these lines can be made only by the $p+56$ Fe reaction.

We have also observed structure in the lineshapes of the 4.44-MeV gamma rays from the ${ }^{12} \mathrm{C}\left(\mathrm{p}, \mathrm{p}^{\prime}\right)^{12} \mathrm{C}$ reaction. At $90^{\circ}$, these lines are peaked at the center at $E_{\mathrm{p}}=5.6 \mathrm{MeV}(\mathrm{lab})$, become flat-topped at $7 \mathrm{MeV}$, and exhibit a pronounced minimum (i.e., there is a symmetric double peak) above $13 \mathrm{MeV}$. This minimum has been observed previously at. $23 \mathrm{MeV} .1$ It is the result of the Doppler shift and the selective population of magnetic sublevels of the $2^{+}, 4.44-\mathrm{MeV}$ state.

An angular distribution table to support two or more $\mathrm{Ge}(\mathrm{L} i$ ) detectors is presently being constructed. It will be used to determine total cross sections and variation of the shape with angle.

1. J.J. Kolata, R. Auble, and A. Galonsky, Phys. Rev. 162, 957 (1967).

6.2 Deuterium Production and Li Destruction Cross Sections

D. Bodansky*

A powerful argument that the universe is closed is based on consideration of the present observed galactic deuterium abundance together with analyses of the conditions necessary to produce this $D$ in the big bang. 1 The argument is contingent upon the assumption that no alternative sites exist. for D production.

Alternative sites have been suggested, including envelopes of exploding supernovae 2 or of even more massive objects. 3 However, any post big-bang model is faced with the following difficulty: Under plausible conditions for deuterium production, lithium will be copiously produced in $\alpha+\alpha$ reactions, helium having been produced in the big bang. The observed present ratio of lithium to deuterium in the galaxy is only $2.5 \times 10^{-4}$ by mass, and the simplest of calculations indicate that the ${ }^{7} \mathrm{Li} / \mathrm{D}$ production ratio will be substantially in excess of this.

Examination of the ${ }^{7} \mathrm{Li} / \mathrm{D}$ ratio therefore offere the prospect of providing a very general exclusion of post big-bang sites for deuterium production. To validate this exclusion quantitativcly, it is necessary to consider the full chain of reactions, including not only those reactions in which $D$ and ${ }^{7} \mathrm{Li}$ are produced, but also those in which they are lestroyed.

We have expiored this problem in the specific context of the colgate model for deuterium production in supernovae shock waves. ${ }^{2}$ Two principal conclusions emerge from the calculations: (a) With any reasonable estimate of the magnitudes of the relevant cross sections, the calculated Li/D ratio far exceeds the observed galactic ratio, unless it is assumed that the cooling of the supernova envelope proceeds at no more than about half the rate suggested by the Colgate model. ${ }^{4}$ (b) At this slower rate of cooling, the final ${ }^{7} \mathrm{Li} / \mathrm{D}$ ratio is quite sensitive to the cross section for the destruction of $\mathrm{Li}$ in $\mathrm{p}+{ }^{7} \mathrm{Li}$ 
reactions. Specifically, if the adopted cross section is varied by $\pm 40 \%$ from the optical model estimate ${ }^{5}$ the $\mathrm{Li} / \mathrm{D}$ ratio varies by about a factor of $\mathrm{six}$.

Details of the Colgate model for deuterium production in supernovae shock waves have been the subject of substantial criticism, and it is possible that $D$ production in supernovae can be excluded without considering the ${ }^{\mathrm{Li}} \mathrm{D}$ ratio. 6 Nevertheless, we expect the foregoing conclusion to apply quite generally to any post big-bang site: if conditions are such as to offer a chance that the calculated $7_{\mathrm{Li} / \mathrm{D}}$ ratio will be acceptably low, the actual ratio will be strongly dependent upon the cross section for destruction of ${ }^{7} \mathrm{Li}$ (and ${ }^{7} \mathrm{Be}$ ) in proton induced reactions.

It. therefore would be valuable to obtain experimental data on the total cross section for $\mathrm{p}+{ }^{7} \mathrm{Li}$ reactions over an energy range extending from threshold up to at least several tens of $\mathrm{MeV}$ and preferably higher. Measurement of all outgoing modes might appear to be a formidable task. However, for so light a nucleus the total cross section can be found from the relation

$$
\sigma_{\text {Tot }}=(t \Phi)^{-1} \sum_{i}\left(Z_{i} / Z_{T}\right) \int N_{i}(\theta) d \Omega
$$

where $t$ is the target thickness, $\Phi$ is the incident proton flux, $N_{i}$ denotes the number of particles detected with charge $\mathrm{Z}_{i}$; and $\mathrm{z}_{\mathrm{T}}$. is the total charge ( 4 in this case). Thus, in principle, the total reaction cross section summed over all channels can be found using a single counter telescope which need only determine the charge of each detected particle. Of course, were this approach to be adopted, it would be necessary to make subtractions for elastic scattering and inelastic scattering to bound states, and to give special consideration to the ${ }^{7} \mathrm{Li}(\mathrm{p}, \mathrm{n}){ }^{7} \mathrm{Be}$ cross section and the fate of the $7_{\mathrm{Be}}$.

* This work was carried out largely during sabbatical leave at the University of Oxford.

1. J.R. Gott, J.E. Gunn, D.N. Schramm, and B.M. Tinsley, Astrophys. J. 194, $543(1974)$.

2. $\quad$ S.A. Colgate, Astrophys. J. 187, 321 (1974); 195, 493 (1975).

3. F. Hoyle and W.A. Fowler, Nature 241, 384 (1973).

4. The same qualitative conclusion has been reported in a comprehensive treatment of light element production in supernovae by R.I. Epstein, W.D. Arnett, and D.N. Schramm, Astrophys. J., to be published.

5. M. Epherre, B. Gaffet, T.P. Meyer, and H. Reeves, Ann. Rev. Astron. and Astrophys. 12, 464 (1974); and B. Gaffet, private communication.

6. T.A. Weaver and G.F. Chapline, Astrophys. J. 192, L57 (1974); T.A. Weaver, $\mathrm{Ph} . \mathrm{D}$. Thesis, University of California, Livermore, 1975 (UCRL-51832). 


\section{FUNDAMENTAL SYMMETRIES IN NUCLEI}

$7.1 \quad$ Parity Mixing in ${ }^{18} \mathrm{~F} *$

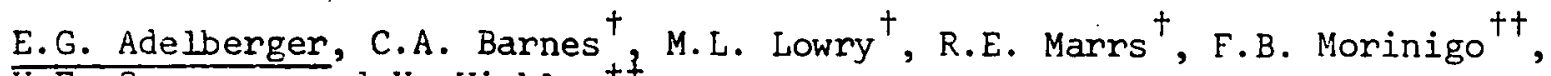
H.E. Swanson, and H. Winklert

The isospin structure of the parity violating interaction is interesting because the different components $(\Delta T=0, \Delta T=1$ and $\Delta T=2)$ carry information about separate elements of the weak current. For example in the Cabbibo (charged currents only) model of the weak interaction, the $\Delta T=1$ component of the $\mathrm{N}-\mathrm{N}$ force is suppressed by a factor of 220 with respect to the $\Delta \mathrm{T}=0$ force. However, if the neutral weak currents recently discovered in high energy neutrino induced reactions violate parity, they would enhance the $T=1$ parity violating $\mathrm{N}-\mathrm{N}$ force by a factor of $\mathrm{ll}$ over the Cabbibo model. 1 Since there are theories where the neutral weak current violates parity (Weinberg-Salam ${ }^{2}$ ) and others where the currents are parity conserving (Fritzsch, Gell-Mann and Minkowski ${ }^{3}$ ) a measurement of the $\Delta \mathrm{T}=1$ interaction is clearly very interesting. Howeven as yet there is no successful measurement of the $\Delta \mathrm{T}=1 \mathrm{PV}$ force. Previously observed effects 4,5 in light nuclei (in 160 and $n+p)$ were completely insensitive to the $\Delta T=1$ force. (Parity violation in heavy nuclei is effectively insensitive to the $\Delta \mathrm{T}=1$ force since it is strongly suppressed by isospin vector coupling to the neutron excess.) We have recently completed a measurement ${ }^{6}$ of parity mixing in ${ }^{19} \mathrm{~F}$. This system is outstanding because of its great simplicity, being, with the exception of the $\mathrm{N}+\mathrm{N}$ system, the most theoretically tractabie case of nuclear parity mixing yet investigated experimentally. In addition, the $1 \hat{q}_{\mathrm{F}}$ mixing is sensitive to the isovector as well as the isoscalar violating $\mathrm{N}-\mathrm{N}$ force. However our present errors are too large to distinguish whether the $\Delta T=1$ force is enhanced or not, since it must be detected "on top of" the $\Delta T=0$ force which is also present in $19 \mathrm{~F}$.

Clearly it would be nice to find a system where the parity violation is purely $\Delta \mathrm{T}=1$. Apparently the most promising opportunity for such a measurement occurs in $18_{\mathrm{F}}$ which seems ideally suited for the task (see Fig. 7.1-1.). There a nearly degenerate doublet of $\mathrm{J}=0$ levels is found with a splitting of only $39 \mathrm{keV}$. The $1042 \mathrm{keV} \mathrm{J}^{\pi}, \mathrm{T}=0^{+}, \mathrm{I}$ level will only
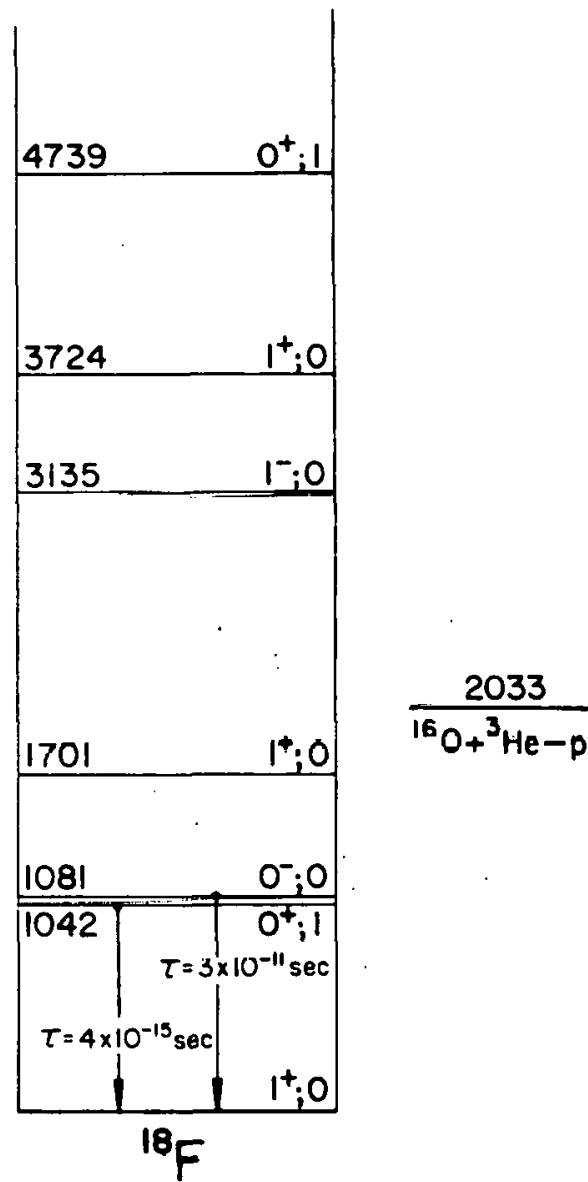

Fig. 7.1-1. Diagram of the low lying $J=0$ and $J=1$ levels of $18 \mathrm{~F}$. 
mix with the $1081 \mathrm{keV} \mathrm{J}^{\pi}, \mathrm{T}=0^{-}, 0$ level under the action of a $\Delta \mathrm{T}=1 \mathrm{PV}$ weak force. Consider the parity properties of the 1081 and $1042 \mathrm{keV} \gamma$-ray transitions. Under a $\Delta \mathrm{T}=1 \mathrm{PV}$ interaction the $\mathrm{O}^{+}$and $\mathrm{O}^{-}$levels will $\mathrm{mix}$ so that

$$
\begin{aligned}
& |1081\rangle=|-\rangle+\varepsilon|+\rangle \\
& |1042\rangle=|+\rangle-\varepsilon|-\rangle
\end{aligned}
$$

where $=\frac{\left\langle-\left|\mathrm{H}_{\mathrm{PV}}\right|+\right\rangle}{.39 \mathrm{keV}}$.

The transitions to the ground state, $|0\rangle$ are then

$$
\begin{aligned}
& \langle 0|E l+M I| 1081\rangle=E I+\varepsilon \cdot M I \\
& \langle 0|E l+M I| 1042\rangle=M I-\varepsilon \cdot E l
\end{aligned}
$$

where

$$
E l=\langle 0|E l|-\rangle \text {. }
$$

$$
M 1=\langle 0|M I|+\rangle \text {. }
$$

The isospin forbidden El transition is very retarded $\tau_{1081}=3 \times 10^{-11} \mathrm{sec}$, while the isospin favored Ml transition is very fast $\tau_{1042}=4 \times 10^{-15} \mathrm{sec}$. From these lifetimes we have Ml $100 \mathrm{El}$. The PV circular polarization (CP) of the 1081 and $1042 \mathrm{keV}$ gamma rays is therefore

$$
\begin{array}{ll}
\mathrm{CP}_{1081} & 2 \varepsilon \cdot \frac{\mathrm{Ml}}{\mathrm{El}} \cdot 200 \varepsilon \\
\mathrm{CP}_{1042} & 2 \varepsilon \cdot \frac{\mathrm{El}}{\mathrm{MI}} \cdot \frac{\varepsilon}{50} \cdot
\end{array}
$$

Using shell model wave functions Gari et al. ${ }^{7}$ predict that $\mathrm{CP}_{1081}=3.6 \times 10^{-4}$ in the Cabbibo model, and $\mathrm{CP}_{1081}=5.7 \times 10^{-3}$ in the Weinberg-Salam model.

We are performing an experiment designed to test if $\mathrm{CP}_{1081}$ is as large as predicted by theories with neutral current enhancements. The CP is determined by a pair of transmission type Compton polarimeters viewed by $15 \% \mathrm{Ge}(\mathrm{Li}$ ) detectors feeding two fast ADC's. Since we have good energy resolution we are able to make a relative measurement of $\mathrm{CP}_{1081}$ by comparing it to $\mathrm{CP}_{1042}$ which acts as a normalizer. The basic deslgr for eliminating systematic errors is quite similar to that employed in the Seattle measurement of parity mixing in ${ }^{19} \mathrm{~F} .6$ In both experiments there are 2 detectors, 2 gamma rays (one a normalizer which should have no true PV effects) and two conditions between which one switches repetitively (in this case the direction of $\vec{B}$ in the polarimeters). The system is highly effective at suppressing cxperimental asymmetries.

The experimental geometry is shown in Fig. 2. The ${ }^{18}$ F activity is produced by the 160 ( ${ }^{\mathrm{He}, \mathrm{p}}$ ) reaction. A thick $\mathrm{H}_{2} \mathrm{O}$ target is bombarded by a $20 \mu \mathrm{A}$ 4.0 MeV $3 \mathrm{He}^{+}$beam from the CSULA KN4000 Van de Graaff accelerator. Some interesting technical problems had to be solved in order to handle the high beam currents required to achieve statistical accuracy. Distilled water in the target circulates rapidly in a closed loop past an entrance window of $1 \times 10^{-4} \mathrm{~cm}$ thick nickel foil. The circulation is necessary to dissipate the beam power ( 80 watts) cool the $\mathrm{Ni}$ foil (the power density in the $\mathrm{Ni}$ foil is $\mathrm{il}$ Megawatt/ $\mathrm{cm}^{3}$ and to carry away the intense $\beta^{+}$activity which would otherwise flood our counters with $511 \mathrm{keV} \gamma$-rays. We chose the $160\left({ }^{3} \mathrm{He}, \mathrm{p}\right)$ reaction to produce the ${ }^{18} \mathrm{~F}$ activity because we obtain good yield of the 1081 and $1042 \mathrm{keV} \gamma$-rays while generating almost no neutrons. We are below the neutron thresholds for ${ }^{3} \mathrm{He}$ on $160,1_{\mathrm{H}}$ and $2 \mathrm{H}$. As a result we have a very intense source of $18 \mathrm{~F} \gamma$ rays (total $\gamma$ ray dose 


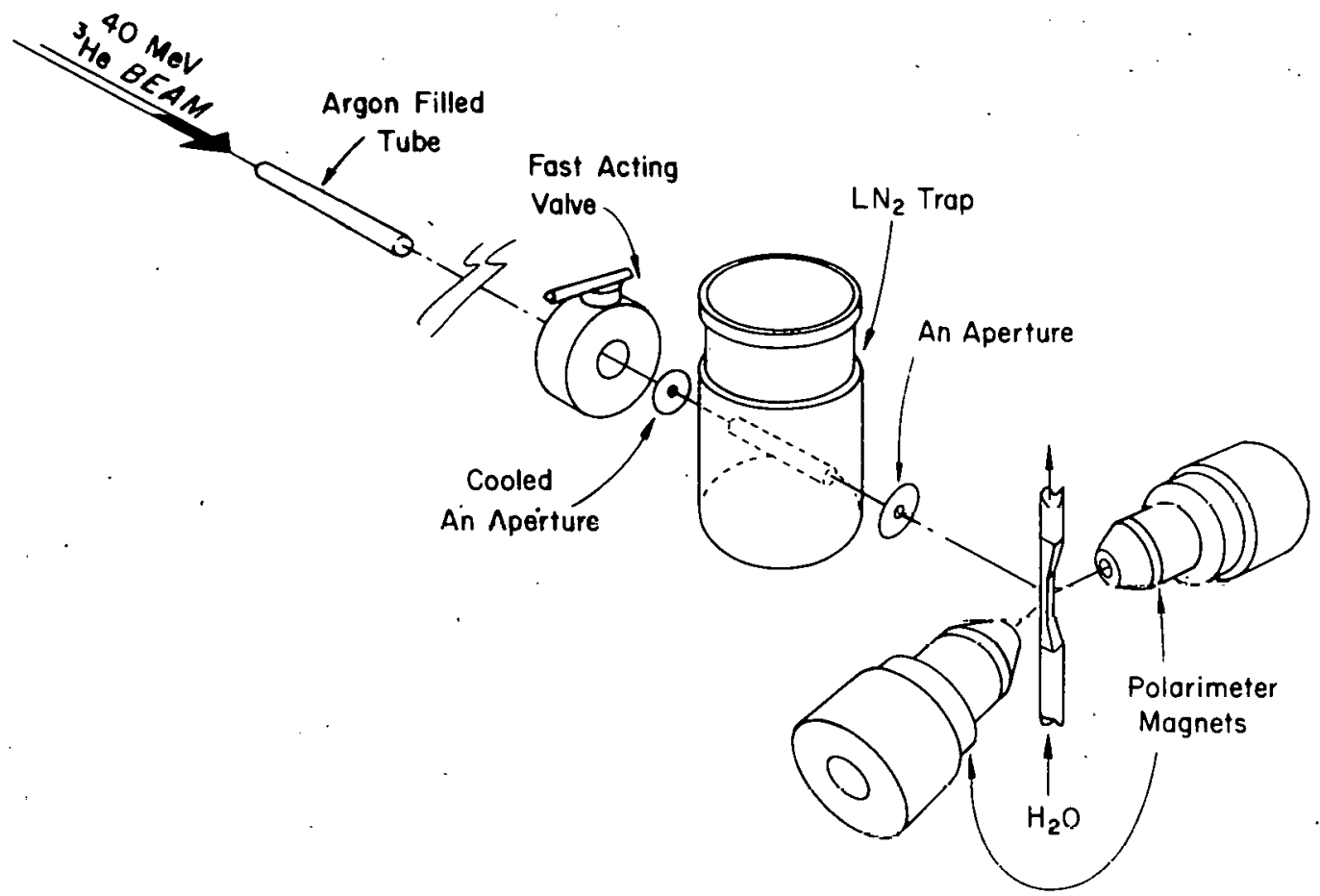

Fig. 7.1-2. Partial schematic diagram of the experimental geometry for the ${ }^{18} \mathrm{~F}$ experiment.

Ul R/hr $15 \mathrm{~cm}$ from the target) while maintaining such a low neutron yield that we can expect the $\mathrm{Ge}(\mathrm{Li})$ counters to survive during the many weeks of bombardment required to obtain statistical accuracy. Before entering the $\mathrm{H}_{2} \mathrm{O}$ cell the ${ }^{3} \mathrm{He}$ beam passes through a tube containing Ar gas which multiple scatters the beam. This prevents intense spots in the boam current dislribution on the target which could cause premature rupturing of the thin $\mathrm{Ni}$ foils. All In-line cold trip plus a system of fast acting automatic vacuum and water valves triggered by an ionization type vacuum gauge protects the accelerator vacuum in event of a rupture of the thin $\mathrm{Ni}$ foil. Under our conditions foils last $\sim 1 / 2$ day before rupturing.

We have constructed twọ highly cfficienl clrcular polarimeters. 'The flux density in the Hyperco 35 cores is $\sim 23$ kgauss while the magnetic field at the target is only a few gauss. The polarimeters incorporate heavy metal $\left(\rho=17 \mathrm{gm} / \mathrm{cm}^{3}\right)$ shielding in order to minimize the flux of scattered $\gamma$-rays in the $\mathrm{Ge}(\mathrm{Li}$ ) detectors. Our polarimeters are quite thick -- attenuating $I \mathrm{MeV} \gamma$ rays by a factor of $\sim 50$-since we operate in the count rate limited regime ( w $4 \times 10^{4}$ counts $/ \mathrm{sec}$ in each. $\mathrm{Ge}(\mathrm{L} 1))$. In spite of this large attenuation the gamma ray spectra are quite good (see Fig. 3) because of the well designed shielding. The polarimeter magnetization can be reversed on $\sim 100 \mathrm{msec}$. The magnetizations in the two polarimeters are always parallel. The fields are reversed repetitively by a precision timer. The Ge(Li) pulses are digitized in two $100 \mathrm{MHz}$ ADC's whose outputs are routed into different regions of memory according to the magnetization direction.

The CP of the $\gamma$-rays is extracted with the expression 


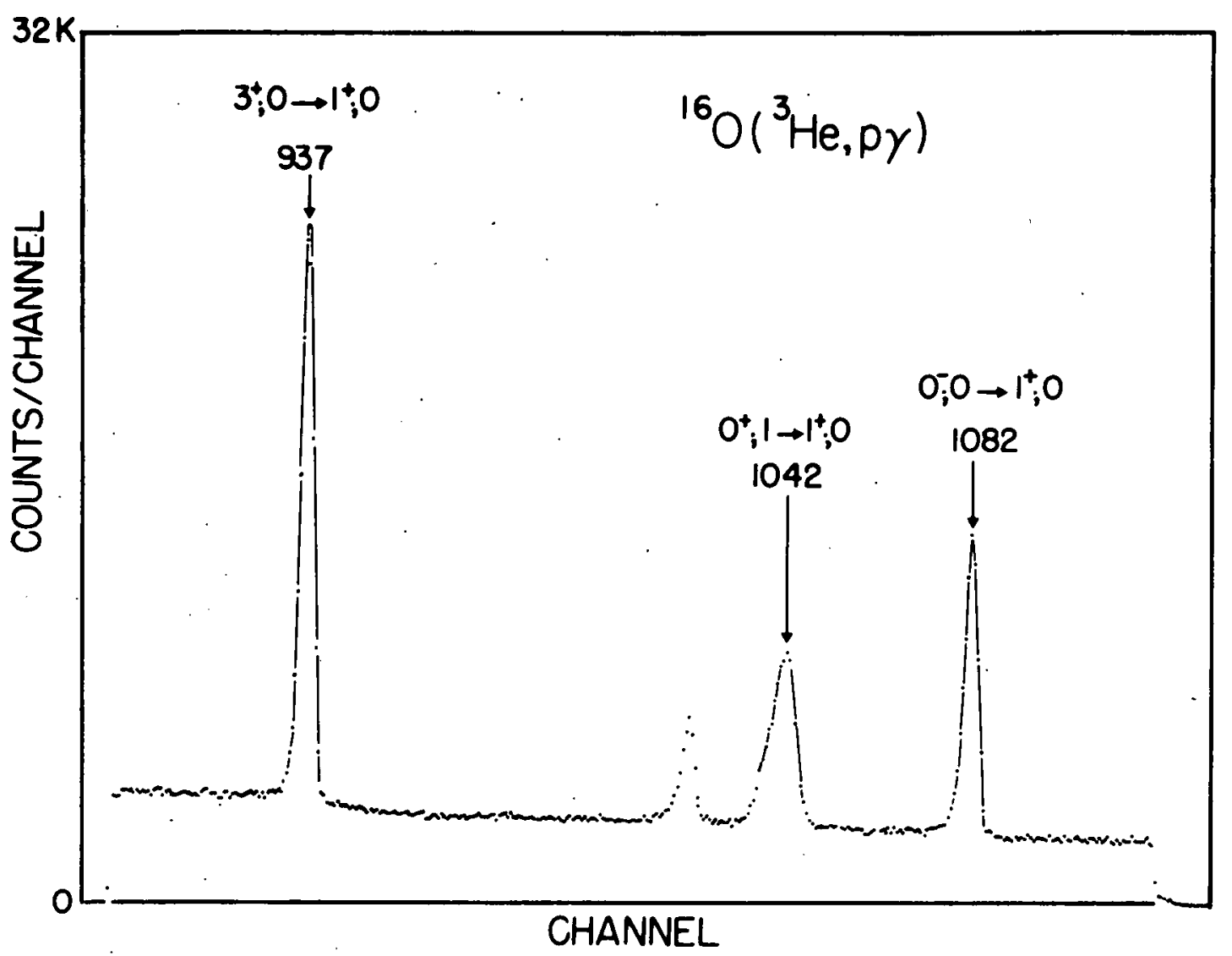

Fig. 7.1-3. Spectrum of $\gamma$-rays in one of our transmission polarimeters. All the gamma ray peaks are from transitions in. $18_{\mathrm{F}}$. The continuous'background arises primarily from the Compton plateau of the $5.2 \mathrm{MeV}$ gamma rays from $16 \mathrm{O}\left({ }^{3} \mathrm{He}, \alpha\right)$.

$$
C P=\left[\frac{L^{+}}{R^{+}} \frac{R^{-}}{L^{-}}-1\right] / 4 \pi
$$

where $n=1.7 \times 10^{-2}$ is the analyzing efficiency of the polarimeters and $\mathrm{L}^{+}$and $R^{+}$refer to counting rates on the left (L) and right ( $R$ ) Ge(Li) counters during the magnetization directions + and -.

Our data based on over a month of bombardment show statistically significant apparent CP's for several ${ }^{18} \mathrm{~F} \gamma$ rays at the $1 \%$ level. We believe these are due to instrumental asymmetries and are making a systematic study of possible effects. Measurements of stray magnetic fields show that the spurious asymmetries are not due to effects of the stray fields on the beam, residual nuclei or the $\mathrm{Ge}(\mathrm{Li})$ detectors. Tests with an intense ${ }^{60} \mathrm{Co}$ source replacing the $\mathrm{H}_{2} \mathrm{O}$ target reveal some instrumental asymmetries which are not yet understood. Investigation of these effects is continuing.

* Work supported in part by the NSF (Caltech) and the State of California (CSULA). 
$+\quad$ Present address: California Institute of Technology, Pasadena, CA

t† Present address: California State University at Los Angeles, CA

1. M. Gari and J.H. Reid, Phys. Lett. 53B, 237 (1974).

2. S. Weinberg, Phys. Rev. Lett 19, 1264 (1967); 27, 1688 (1971); A. Salam

in Elementary Particle Theory (Nobel Symposium No. 8) ed. N. Svartholm

(John Wiley, New York, 1969).

3. H. Fritzsch, M. Gell-Mann and P. Minkowski, Phys. Lett. 59B, 256 (1975).

4. K. Neubeck, H. Schober and H. Wäffler, Phys. Rev. C 10, 370 (1974).

5. U.M. Lobashov et al., Nucl. Phys. A197, 241 (1972)

6. E.G. Adelberger, H.E. Swanson, M.D. Cooper, J.W. Tape, and T.A. Trainor Phys. Rev. Lett. 34, 402 (1975).

7. M. Gari, J.B. McGrory and J. Offerman, Phys. Lett. 55B, 277 (1975).

7.2 A New Experiment to Measure the Parity Mixing in ${ }^{19} \mathrm{~F}$

E.G. Adelberger, H.E. Swanson, and T.A. Trainor

Our previous measurement ${ }^{l}$ of the parity mixing between the ground $\left(1 / 2^{+}\right)$ and first excited $\left(1 / 2^{-}\right)$states of ${ }^{19} \mathrm{~F}$ continues to receive attention because the nuclear physics involved in the parity mixing is unusually straightforward. With the single exception of the $n+p$ system it is more tractable theoretically than any other case yet investigated experimentally. The magnitude of our published value ${ }^{1}$ of the parity mixing is in reasonable agreement with calculations ${ }^{2-4}$. Unfortunately our present errors are too large to detect whether or not there is a neutral current enhancement in $19 \mathrm{~F}$ because it must be detected "on top of" the large $T=0$ mixing from the charged currents. However Desplanques 5 and Box, Gabric and McKellar 4 have recently calculated the sign of the parity violating asymerry in ${ }^{19} \mathrm{f}^{\prime}$ and found that the measured sign disagrees with that expected if the effect were dominated by the $\Delta \mathrm{T}=0$ amplitude. For these reasons, it would be very interesting to have a more precise measurement of the parity violation in $19 \mathrm{~F}$.

We are currently developing apparatus for an improved measurement of the parity mixing in ${ }^{19} \mathrm{~F}$. The primary improvements come from extensive modifications to our polarized ion source. Recent developments (see Sec. 2.1 of this report) have greatly increased the reliability and intensity of our ion source. This greatly reduces the running time required for improved statistical accuracy. We are now constructing a Los Alamos style fast spin reversal system 6 based on a "spin filter". This will allow us to practically double our $19 \mathrm{~F}$ polarization since we performed our last measurement ${ }^{1}$ using the adiabatic field reduction scheme in order to reduce unwanted correlations between the spin state and other properties of the polarized heam. With the Los Mlamos style spin fllpper undesired modulation of the polarized beam will be reduced to a negligible level. This permits us to remove the on-line "correlation analyzer" which was placed upstream of the target. This change will reduce the background in the $\mathrm{Ge}(\mathrm{Li})$ detectors by eliminating the multiple scattering which sprayed beam on collimators. We are constructing a digital feedback system to stabilize the proton spin angle to $0.1^{\circ}$ (see Sec. 3.8 of this report). This is based on a carbon polarimeter located immediately before the ${ }^{22} \mathrm{Ne}$ gas cell used to produce the ${ }^{19} \mathrm{~F}$ activity. Our previous work ${ }^{l}$ showed that uncontrolled variations of $\sim \pm 1^{\circ}$ in the proton 
spin angle were the largest source of systematic errors. This should be virtually. eliminated in our new experiment.

1. E.G. Adelberger, H.E. Swanson, M.D. Cooper, J.W. Tape and T.A. Trainor, Phys. Rev. Lett. 34, 402 (1975).

2. M. Gari, A.H. Huffman, J.B. McGrory and R. Offenman, Phys. Rev. C 11, 1485 (1975).

3. M.A. Box and B.H.J. McKellar, Phys. Rev. C 11, 1859 (1975).

4. M.A. Box, A.J. Gabric and B.H.J. McKellar, to be published.

5. B. Desplanques, private communication (1976).

6. "Alternating Polarization at $1000 \mathrm{~Hz}$ in a Lamb-Shift $\mathrm{H}^{-}$Source", J.L. McKibben and J.M. Potter, contribution D23 in Proc. of Fourth Int. Symposium on Polarization Phenomena in Nuclear Reactions, Zurich 1975 , to be published.

7.3 A Redetermination of the Effective ${ }^{19} \mathrm{~F} *$ Polarization in the ${ }^{19} \mathrm{~F}$ Parity Mixing Experiment

E.G. Adelberger, H.E. Swanson, and T.A. Trainor

Our measured value for $\delta$, the parity violating asymmetry in ${ }^{19} \mathrm{~F}$, is equal to the observed asymmetry divided by the net $1{ }^{2} F^{*}$ polarization (see $\operatorname{Refs}$. 1 and 2). The dominant uncertainty in our previous measurementl,2 of the net $19 \mathrm{~F}^{*}$ polarization lay in the analyzing power, $n$, of our transmission polarimeter. This resulted from our uncertainties in the differential cross sections for coherent scattering of $110 \mathrm{keV} \gamma$-rays from $\mathrm{Fe}$. The recent publication of a careful calculation of coherent scattering cross sections ${ }^{3}$ has prompted us to make a redetermination 'of the net ${ }^{19} \mathrm{~F} *$ polarization.

Coherent scattering angular distributions are given in Ref. 3 for low energy $\gamma$-rays including 100 and $150 \mathrm{keV}$ and a straightforward interpolation made to $110 \mathrm{keV}$. A series of exponential terms found to reasonably approximate this functional form was inserted in our existing Monte Carlo code (described in the 1974 Annual Report) ${ }^{2}$ and a calculation taking about 8 hours made on the Nuclear Physics Laboratory's SnS 930 computer. The calculation resulted in an analyzing power, assuming $100 \%$ electron polarization of $n=-3.50 \pm .14 \times 10^{-2}$ where errors were determined from fluctuations in intermediate printouts.

To better determine the actual fraction of electrons polarized, the magnet flux in the polarimeter was remeasured using an improved integration built around an Analog Divice Op-Amp. The output of a sense coil wound around the absorber iron was integrated, while switching the direction of flux in the polarimeter, resulting in an output proportional to twice the net flux. The integrator was calibrated using a flip coil and the NMR measured field of the accelerator's $90^{\circ}$ magnet. Fields of $1.927 \mathrm{~T}$ and $2.005 \mathrm{~T}$ were obtained for winding currents of 0.8 amps and 1.2 amps respectively. Assuming that the measured field comes only from the electron spin, this corresponds to polarizing 2.00 and 2.08 electrons respectively out of 26 . In our previously reported value we assumed 2 electrons were polarized. 


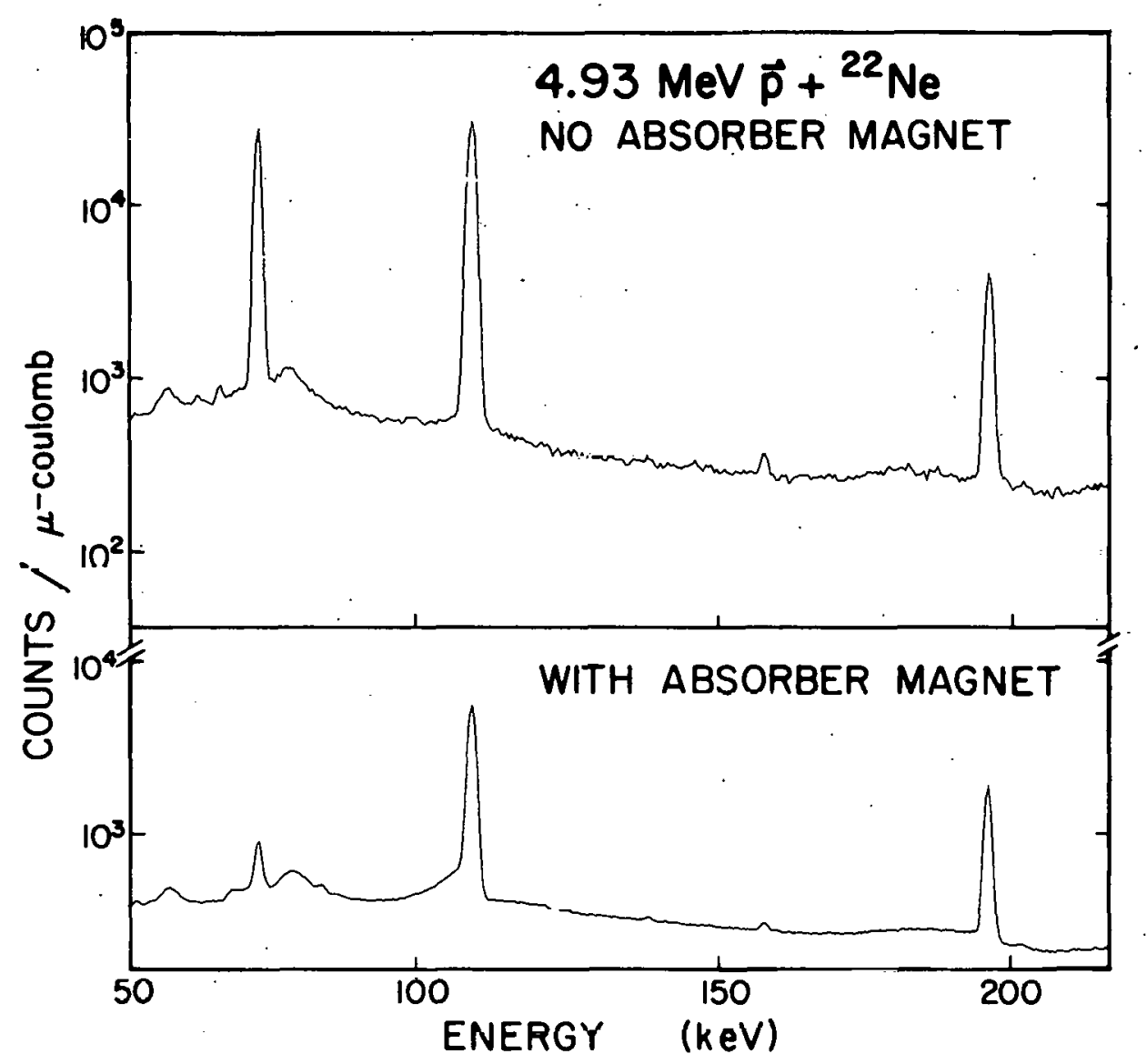

Fig. 7.3-1. Typical planar Ge(Li) spectrum shown with and without polarimeter absorber magnet in place.

Finally, additional running time was obtained measuring the counting asymmetry to further improve statistical uncertainties. Figure 7.3-1 shows typical spectra obtained with and without the polarimeter in place. The polarimeter magnet was run at a lower current this time (.8 vs 1.2 amps) resulting in a slightly lower analyzing power but also an observable reduction in the instrumental offset, presumably due to weaker fields outside the iron whish perturb the beam position.

The results of this and the previous determination are shown in 'Fig. 7.3-2 where the normalized circular polarization is obtained by dividing the asymmetry in counting rates by the appropriate analyzing power and beam polarization. The polarization transferred from the beam to $19 \mathrm{~F} *$ nuclei is obtained from the difference in the spin-right and spin-left measurements to cancel instrumental effects, and found to be $k_{x \bar{x}}=-0.55 \pm .07$. The change from our previous value $k_{x \bar{x}}=-0.73 \pm$ 0.15 is essentially entirely due to the revision in the analyzing power of our polarimeter.

Let us summarize: Our new measurements of the polarimeter magnetization and the circular polarization dependent asymmetry. $A_{\mathrm{CP}}$ are in good agreement with 
with our earlier work. However we find that the analyzing power of our circular polarimeter is larger than calculated in our earlier work. The effect of this is to increase our value for the parity violating asyminetry, $\delta$, from $\delta=-(18 \pm 9)$ $\times 10^{-5}$ to $\delta=-(24 \pm 12) \times 10^{-5}$.

1. E.G. Adelberger, H.E. Swanson, M.D. Cooper, J.W. Tape, and T.A. Trainor, Phys. Rev. Lett. 34, 402 (1975).

2. Nuclear Physics Laboratory Annual Report, University of Washington (1974), p. 52 .

3. R.T. Brown in Atomic Data and Nuclear Data Tables, Vol. 15, No. 2 (Feb. 1975), p. 135.

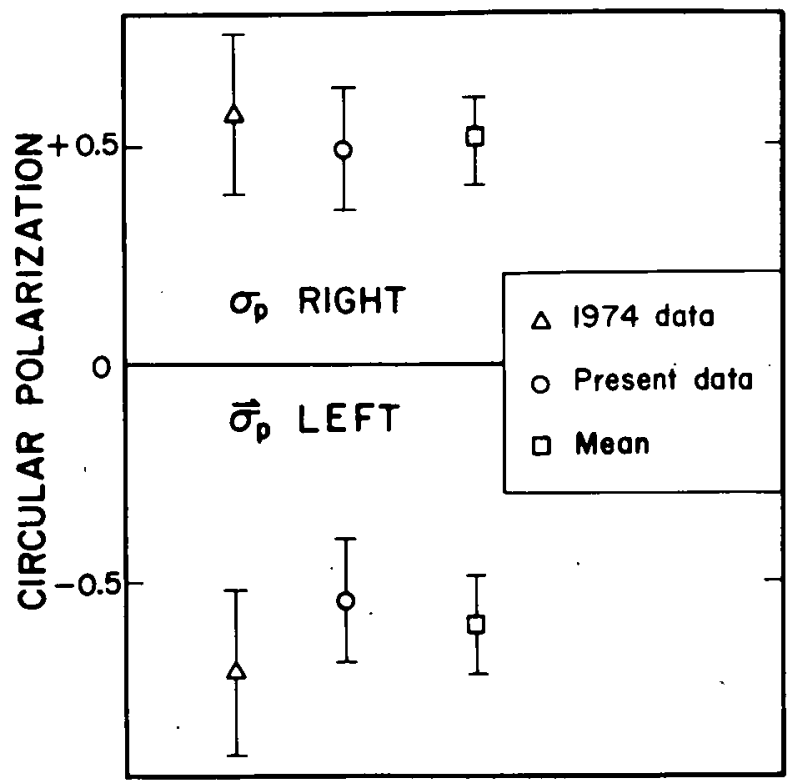

Fig. 7.3-2. Circular polarization of 110 $\mathrm{keV} \gamma$-radiation from polarized $19_{\mathrm{F}} *$ (normalized to $100 \%$ beam polarization) is shown for right and left beam polarizations. 


\section{NUCLEAR STRUCTURE}

8.1 Gamma Decays of the Lowest $\mathrm{T}=3 / 2$ Levels in ${ }^{9} \mathrm{Be}$ and ${ }^{9} \mathrm{~B}$

E.G. Adelberger, P.A. Dickey, P. Dyer, and K.A. Snover

Last year we reported preliminary results of a search for isospin violating effects in the gamma decays of the lowest $T=3 / 2$ levels in ${ }^{9} \mathrm{Be}$ and ${ }^{9} \mathrm{~B}$. Since then, considerable effort has been expended in improving the quality of the spectra, and although there still are a number of peripheral measurements to be made, the coincidence data for the lowest $\mathrm{T}=3 / 2$ levels is complete.

The nuclear electromagnetic current is usually assumed to transform like the charge under isospin rotations, with the result that isoscalar and isovector, but not isotensor terms are present in the gamma-ray transition operator. The vanishing of the isoscalar interaction for $|\Delta T|=1$ electromagnetic transitions implies that corresponding gamma transitions from isospin mirror levels should be identical. An isotensor term, if present, would induce an asymmetry in these mirror decay rates through its interference with the dominant isovector amplitude. The sensitivity with which one can hope to observe $\Delta T=2$ currents through companisons of mimor $\gamma$-decay rates is limited on the one hand because an intermediate meson is needed to couple a $\Delta \mathrm{T}=2$ current to a $\mathrm{T}=1 / 2$ nucleon, 1 and on the other hand because asymmetries can also arise from Coulomb induced distortions of nominally analogous nuclear states. The Coulomb effects must be understood before one can attribute an asymmetry to a $\Delta \mathrm{T}=2$ current. Nevertheless the effort is justified by the tremendous interest in testing such a fundamental property of the electromagnetic interaction in nuclei. $A \Delta T=2$ electromagnetic radiation of substantial intensity would prove the existence of meson exchange currents. The coulomb effects are also very interesting because of their evident role in the nuclear structure of mirror states.

Mirror ML gamma decays of the lowest ' $l=3 / 2$ levels in $4 N+1$ nuclei provide a good experimental test of the $|\Delta \mathrm{T}|=1$ selection role because the isospin purity of the $\mathrm{T}=3 / 2$ levels is rather well known and the high energy gamma decays are strong. The mass $9^{2}$ and mass $13^{3}$ decays have been studied previously by Cocke et al. No asymmetry in the Ml decays was observed, but the large experimental uncertainties precluded a stringent test of the selection rule. Effects in nuclei of a possible isotensor electromagnetic current are expected to be small. Recently Marrs et al. ${ }^{4}$ have remeasured the mass 13 decays with high precision and found no isospin violation in the mirror MI decays, though a large asymmetry in E1 decays was attributed to charge dependent configuration mixing in the first excited states. The precision of Marrs' experiment derived from the $4 \%$ energy resolution of the Nuclear Physics Laboratory 10"x10" NaI spectrometer and the minimization of systematic errors by the use of the same geometry and coincidence electronics for calibration of the NaI detector and for measurement of the $T=3 / 2$ decays in each nucleus. It was decided to apply these same techniques to the mass 9 system.

The reactions ${ }^{7} \mathrm{Li}\left({ }^{3} \mathrm{He}, \mathrm{P}\right){ }^{9} \mathrm{Be}$ and ${ }^{7} \mathrm{Li}\left({ }^{3} \mathrm{He}, \mathrm{n}\right)^{9} \mathrm{~B}$ were used to populate the $\mathrm{T}=3 / 2$ levels and gamma rays were detected in coincidence with protons or neutrons. The gamma decays of interest are indicated in Fig. 8.1-1. Particles were detected 


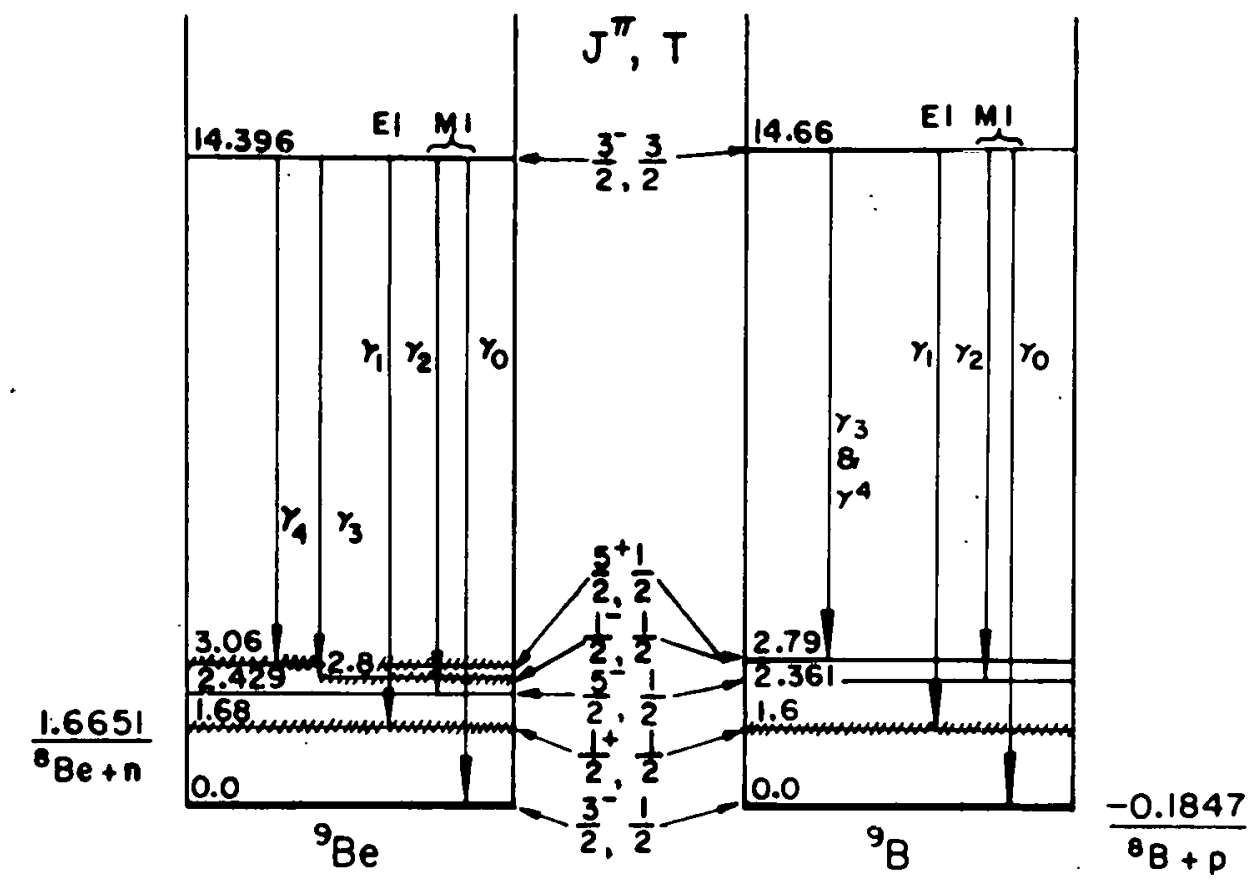

Fig. 8.1-1. Partial level schemes of mass 9 mirror nuclei.

at $0^{\circ}$ and gammas at $125^{\circ}$, a zero of $\mathrm{P}_{2}$, to permit the extraction of total yields. Approximately $200 \mu \mathrm{g} / \mathrm{cm}^{2}$ of ${ }^{7} \mathrm{Li}$ was evaporated simultaneously onto $\mathrm{Ni}$ and $\mathrm{Ta}$ backings; these targets were then transferred to the scattering chamber in vacuum. For the ${ }^{9} \mathrm{Be}$ measurement a $7.5 \mathrm{MeV}{ }^{3} \mathrm{He}$ beam bombarded the $\mathrm{Ni}$ backed target and was ranged out in stopping foils which allowed protons forming the $T=3 / 2$ state to emerge at $0^{\circ}$ with $1.2 \mathrm{MeV}$ of energy. A $13 \mathrm{MeV}$ beam on the Ta backed target was used for $9_{B}$. The bombarding energies were carefully chosen to avoid contamination of the data by gamma rays in coincidence with decay particles from the unbound levels in the mirror nucleus. Protons were detected in a $\Delta E-E$ telescope and neutrons in a plastic scintillator. The neutron energies were deduced from the time of flight (TOF) over a $60 \mathrm{~cm}$ path. The gamma ray detection system was untouched between the two complementary measurements.

Three-parameter coincidence data were event recorded on magnetic tape. In addition, during the $9_{B e}$ runs, proton singles and coincidences were simultaneously accumulated through the same ADC. This permitted absolute $\gamma$-decay branching ratio determinations in ${ }^{9} \mathrm{Be}$ that are free from dead time and beam instability errors. The analogous singles neutron yield for ${ }^{9} \mathrm{~B}$ requires a pulsed ${ }^{3} \mathrm{He}$ beam and will be measured elsewhere. A monitor detector used during the coincidence runs will allow normalization of the neutron singles data when they are obtained.

The mirror $\Delta \mathrm{T}=1$ gamma decay spectra are shown in Fig. 8.1-2 al'ong with least squares fits based upon the response function of the NaI detector for monoenergetic gamma rays. The response function and detector efficiency were determined in a separate $10_{\mathrm{B}}\left(3_{\mathrm{He}}, \mathrm{p} \gamma\right)^{12} \mathrm{C}(15.11)$ coincidence measurement employing the 


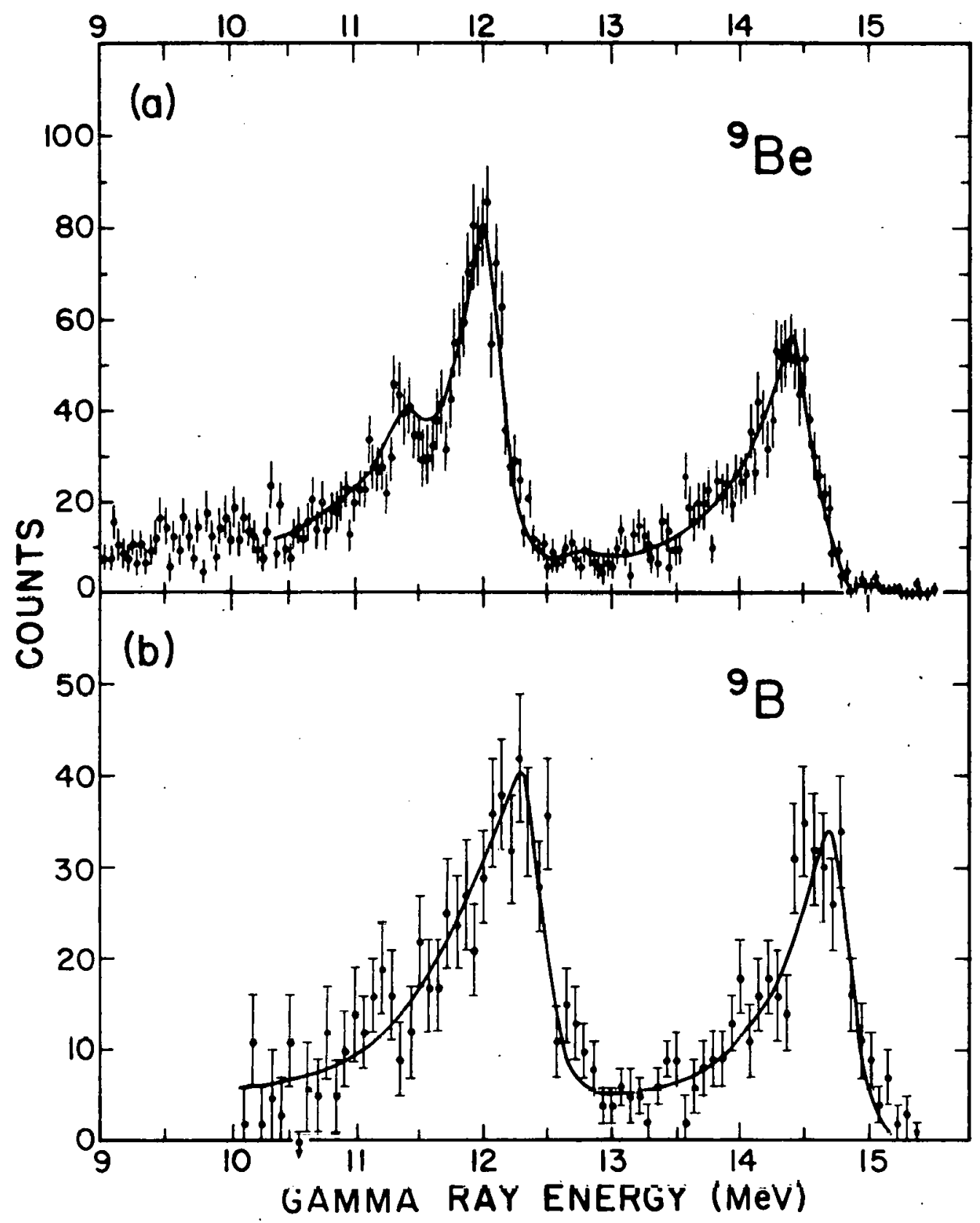

Fig. 8.1-2. Coincidence gamma ray spectra from the decay of lowest $T=3 / 2$ levels in a) $9 \overline{B e}$ and b) $9 \bar{B}$. Solid lines are preliminary fits to the data.

same experimental arrangement as the mass 9 decays. A second lineshape, for 10:5 $\mathrm{MeV}$ gamma rays, was obtained from a ${ }^{27} \mathrm{Al}\left(\mathrm{p}, \gamma_{2}\right)^{28} \mathrm{Si}$ singles spectrum. We then generated the lineshape at intermediate energies by an interpolation procedure.

Early in our analysis we found the ${ }^{9}$ Be spectrum to be inconsistent with the assignment of a significant branch to the $2.8 \mathrm{MeV}, 1 / 2^{-}$state as reported by 
Cocke et al. ${ }^{2}$ our data instead indicated decays to a level nearer $3.1 \mathrm{MeV}$ in ${ }^{9} \mathrm{Be}$. Cocke's poor resolution data would not be inconsistent with this interpretation. We therefore sought an indepent measurement of the level energy from its neutron decay spectrum obtained in coincidence with the $\Delta \mathrm{T}=1$ gamma rays populating the level. We bombarded a $600 \mu \mathrm{g} / \mathrm{cm}^{2}$ ${ }^{7} \mathrm{Li}$ target with a $5.5 \mathrm{MeV}{ }^{3} \mathrm{He}$ beam and recorded $n-\gamma$ coincidences both at a $65 \mathrm{~cm}$ flight path for optimum neutron energy resolution and at $16.5 \mathrm{~cm}$ to accumulate a coincident gamma ray spectrum with good statistics. Unfortunately the kinematic spread in laboratory neutron energy arising from the ${ }^{9} \mathrm{Be}$ recoils was about $500 \mathrm{keV}$. Nevertheless it is clear from the neutron TOF spectrum in Fig. $8.1-3$ that the $T=3 / 2$ gamma decays do not feed any levels with substantial neutron decays to the ${ }^{8} \mathrm{Be}$ ground state. The broad peak in the spectrum is primarily the result of the well known $92 \%$

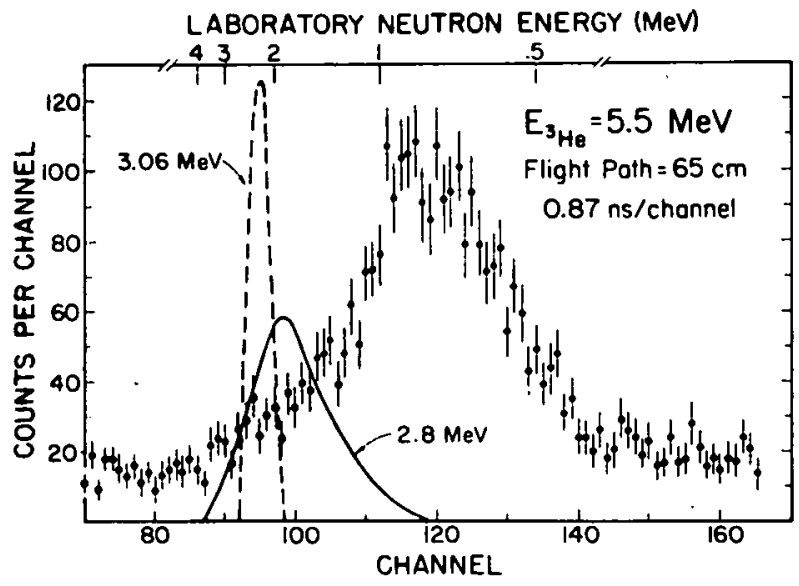

Fig. 8.1-3. Time of flight spectrum for neutrons emitted from states in $9 \mathrm{Be}$ between 2.4 and $3.5 \mathrm{MeV}$. Smooth curves indicate the expected contributions from the known $5 / 2^{+}(3.06 \mathrm{MeV})$ and $1 / 2^{-}$ $(2.8 \mathrm{MeV})$ states if these states were responsible for the counts in the $T=3 / 2$ $\gamma$-decay spectrum near $3 \mathrm{MeV}$ excitation. branch of the $2.43 \mathrm{MeV} 5 / 2^{-}$level into the three-body $2 \alpha+n$ channel. Decays of the known $2.8 \mathrm{MeV}$ or $3.06 \mathrm{MeV}$ states in ${ }^{9} \mathrm{Be}$ through their dominant ${ }^{8} \mathrm{Be}(0)+\mathrm{n}$ mode would necessarily produce tremendous peaks, not seen in the TOF spectrum. The data instead indicate a continuum distribution of neutron energies. Although the analysis is not yet complete, on the basis of the $n-\gamma$ data we tentatively attribute the decay branch to a new state in $9 \mathrm{Be}$ with excitation energy $3.2 \pm .1 \mathrm{MeV}$, width $300 \pm 150 \mathrm{keV}$, and a dominant decay mode into the $2 \alpha+n$ channel. The energy and width were deduced from the gamma ray spectrum. The ( $\left.{ }^{3} \mathrm{He}, \mathrm{p} Y\right)$ data in Fig. 8.1-2 were analyzed under this assumption.

Inelastic electron scattering data ${ }^{5}$ indicate the possibility that two overlapping opposite parity levels of nearly equal width may exist at $3 \mathrm{MeV}$ excitation in ${ }^{9} \mathrm{Be}$ : Although the $2.8 \mathrm{MeV}, 1 / 2$ state is now known to overlap the $5 / 2^{+}$state, its width of $1080 \mathrm{keV}$, a factor of three larger than that of the $5 / 2^{+}$ state, causes some difficulty in identifying this state with the longitudinal $\left(e, e^{\prime}\right)$ excitation cross section. There is evidence from a pronounced shoulder near 3.3 MeV excitation in the data of Bockelman et al. ${ }^{6}$ that the proposed state may be excited via ${ }^{1} 1_{B}(d, \alpha){ }^{9} B$. We intend to pursue our study of this apparent new state by performing a ${ }^{1} l_{B}(d, \alpha n)^{8} B e$ coincidence experiment to obtain a decay neutron spectrum for the $3.2 \mathrm{MeV}$ state, free from the continuum of neutrons due to ${ }^{9} \mathrm{Be}(2.43)$ breakup. In addition the $\gamma$ decays of the second $T=3 / 2$ level at $16.98 \mathrm{MeV}$ in ${ }^{9} \mathrm{Be}$ will be studied to see if there is a significant branch to a state at $3.2 \mathrm{MeV}$. Existence of a transition from the $1 / 2^{-} \mathrm{T}=3 / 2$ level would limit the allowed spin and parity of the new state.

Table 8.1-1 lists some results of the preliminary fits of the data of Fig. 8.1-2. Only the branching ratios for the $r_{0}$ and $r_{2}$ decays are listed 
Table 8.1-1. Mass 9 Gamma Branching Ratios

${ }^{9} \mathrm{Be}$

This work

$\Gamma_{\gamma_{0}} / \Gamma$

$\Gamma_{\gamma_{2}} / \Gamma \gamma_{0}$

${ }^{\mathrm{B}} \mathrm{r}_{2} / \mathrm{B}^{\mathrm{B}} \mathrm{r}_{0}$
$.0160 \pm .0008$

$1.05 \pm .05$

$1.85 \pm .09$ $9_{b}$

This work

Ref. 2

** To be determined after nelitron single measurement (see text).

because the extreme sensitivity of the $\gamma_{3}$ yields to the energy and width of the final states in $9_{\mathrm{Be}}$ and $9_{\mathrm{B}}$ would render such comparisons meaningless; even the $\gamma_{2}$ branching ratio is somewhat sensitive to these parameters. It is seen that within statistical errors, there is no asymmetry in the ratio of $M I$ reduced transitionstrengths for decays to the $5 / 2^{-}$and $3 / 2^{-}$states, $\mathrm{B}_{\gamma_{2}} / \mathrm{B}_{\gamma_{0}}$. If this conclusion persists after final analysis, it will imply a one standard error limit of $2.9 \%$ for the ratio of isotensor to isovector amplitudes in mass 9 in the favorable case in which the relative phases of the amplitudes are opposite in sign for the $3 / 2^{-}$and $5 / 2^{-}$transitions. The absolute ground state radiative widths $\Gamma_{\text {ro }}$ do not depend on this unknown phase, and it is this comparison that is the real test of isospin violation. Thus a high priority is placed on the ${ }^{7} \mathrm{Li}\left({ }^{\mathrm{He}}, \mathrm{n}\right){ }^{9} \mathrm{~B}$ neutron singles measurement needed to obtain the absolute radiative widths in ${ }{ }_{B}$.

1. E.G. Adelberger and D.P. Balamuth; Phys. Rev. Lett. 27, 1597 (1971).

2. J.C. Adloff, K.H. Souw, and C.L. Cocke, Phys. Rev. C 3, 1808 (1971).

3. C.L. Cocke, J.L. Adlott, and P. Chevaliier, Phys. Rev. 176, 1120 (1.968).

4. R.E. Marrs, E.G. Adelberger, K.A. Snover, and M.D. Cooper, Phys. Rev. Lett. $35,202(1975)$.

5. H.G. Clerc, K.J. Wetzel, and E. Spamer, Nucl. Phys: A120, 441 (1968).

6. C.K. Bockelman, A. Leveque, and W.W. Buechner, Phys. Rev. 101, 456 (1956).

\section{8.) Tsnspin Mixing in ${ }^{12}$ s*}

E.G. Adelberger, J.E. Bussoletti, R.E. Marrs ${ }^{\dagger}$, and K.A. Snover

The mixing of ' $\mathrm{l}$ '=0 aid $\mathrm{T}=1$ levels in ${ }^{8} \mathrm{Be}$ and ${ }^{12} \mathrm{C}$ has attracted much attention because the off-diagonal matrix elements are believed to be about 150 and $250 \mathrm{keV}$ respectively, 1,2 while Coulomb forces apparently give matrix elements of only $60 \mathrm{keV} .1,3$ These examples seem to support Negele's analysis of displacement energies which requires a sizable $\Delta T=1$ component of the short-range nuclear force. 4 The significance of these results has led us to reexamine the mixing in $12 \mathrm{C}$. The 15.1 MeV $1^{+}, T=1$ and $12.7 \mathrm{MeV} 1^{+} \mathrm{T}=0$ levels form an interesting system for quantitative studies of isospin violation. ${ }^{3}$ Good wave functions are available and the levels are known to have a very similar space structure. All previous analyses 
of the isospin impurities have assumed simple two state mixing.

$$
\begin{aligned}
& |15.1\rangle=\alpha|1\rangle+\beta|0\rangle \quad \beta=\frac{\left\langle 1\left|\mathrm{H}_{C D}\right| 0\right\rangle}{2400 \mathrm{keV}} \quad \alpha^{2}=1-\beta^{2} . \\
& |12.7\rangle=-\beta|1\rangle+\alpha|0\rangle
\end{aligned}
$$

We examine the validity of this approximation in several applications below.

In the three years since Braithwaite, Bussoletti, Cecil and Garvey ${ }^{2}$ (BBCG) reported that $\left\langle 1\left|\mathrm{H}_{\mathrm{CD}}\right| 0\right\rangle=(250 \pm 50) \mathrm{keV}$ a variety of experiments have been performed to check this result. ${ }^{5-8}$ Unfortunately the different approaches have yielded inconsistent results and none of the experiments by itself is completely convincing, We have obtained experimental results which bear upon the isospin mixing in ${ }^{i 2} \mathrm{C}$ and analyzed electromagnetic and single-nucleon transfer data in $A=12$ using the charge independent shell model calculation ${ }^{9}$ of Cohen and Kurath (CK). We show that there is clear evidence for isospin mixing in the $1^{+}$doublet, but that the magnitude is much smaller than that reported by BBCG. 2

Our coincidence measurements of the decay properties of the 12.7 and 16.1 $\mathrm{MeV}$ levels of ${ }^{12} \mathrm{C}$ were reported in previous Annual Reports. Coincidence measurements of the $\gamma$-ray branching ratios of ${ }^{12} \mathrm{C}(12.7)$ and ${ }^{12} \mathrm{C}(16.1)$ were performed at the University of Washington using the $10_{B}\left(3_{\mathrm{He}, \mathrm{pr}}\right)$ reaction at $4.1 \mathrm{MeV}$ incident energy. Protons were detected in a counter telescope at $0^{\circ}$. Gamma rays were counted in a $25.4 \times 25.4 \mathrm{~cm} \mathrm{NaI}$ spectrometer, usually at $125^{\circ}$. The singles and coincidence data were recorded simultaneously on magnetic tape and sorted later to obtain $\gamma$-decay branching ratios. The system is designed so that dead time corrections to the branching ratio are negligible. The $\gamma$-ray detection efficiency was measured to $\pm 3 \%$ as described in Ref. 10. From coincidence spectra we obtain $\left.\Gamma_{\gamma_{0}}=1.93 \pm 0.12\right) \%, \Gamma_{\gamma_{1}} / \Gamma_{\gamma_{0}}=(0.150 \pm 0.018)$, and $\Gamma_{\alpha} / \Gamma=(97.8 \pm 0.1) \%$ for $12 \mathrm{C}(12.7)$, and $\Gamma_{\gamma_{1}} / \Gamma=(2.42 \pm 0.29) \times 10^{-3}$ for $12 \mathrm{C}(16.1)$. Relative $\gamma$-ray branching ratios of ${ }^{12}$ c. (16.l.) were measured at Caltech using the $163 \mathrm{keV} 1 \mathrm{I}_{\mathrm{B}}(\mathrm{p}, \mathrm{\gamma})$ resonance. Water cooled $\mathrm{Il}_{\mathrm{B}}$ targets were bombarded with 10-15 $\mathrm{\mu A}$ (electrical) of $\mathrm{H}_{3}{ }^{+}$ions. Gamma rays were detected with a $15 \% \mathrm{Ge}(\mathrm{Li})$ detector. From a spectrum on resonance (see Fig. 8.2-1) with $0.1 \mathrm{C}$ of integrated charge we deduce $\Gamma_{\gamma_{0}} / \Gamma_{\gamma}=(3.1 \pm 0.5) \%$, $\Gamma_{\gamma}(16.1 \rightarrow 9.6) / \Gamma_{\gamma_{\gamma}}=(2.0 \pm 0.26) \%$, and $\left.\Gamma_{\gamma}(16.1 \rightarrow 12.7) / \Gamma_{\gamma_{1}}=11.45 \pm 0.25\right) \%$. Off resonance $\gamma$-ray yields were found to be negligible. The efficiency of our detector was measured using the known decay schemes of $56 \mathrm{Co}$ and $23^{\mathrm{Na}}(\mathrm{p}, \gamma)$ resonances at $E_{p}=1318$ and $1416 \mathrm{keV} .11$

In Table 8.2-1 we present some experimental data on radiative widths and single nucleon transfer spectroscopic factors in $A=12$, along with the corresponding values from the CK calculation. 9 We focus first on the $\gamma$-ray results. The $\mathrm{CK}$ calculation is in very good agreement with experiment for 7 out of $9 \gamma$-ray transitions, including all the isovector Ml's, with a maximum discrepancy between theory and experiment of $17 \%$. On the other hand the "isoscalar" Ml transitions from the $12.7 \mathrm{MeV}$ level are faster than expected by a factor of 3 .

Since there is no reason why the CK isoscalar transition speeds should be less accurate than the isovector speeds, we follow Ref. 5 and assume that the anomalously fast "isoscalar" transitions contain an isovector component. This can arise from $T=1$ impurities in either the initial or the final state. We find 


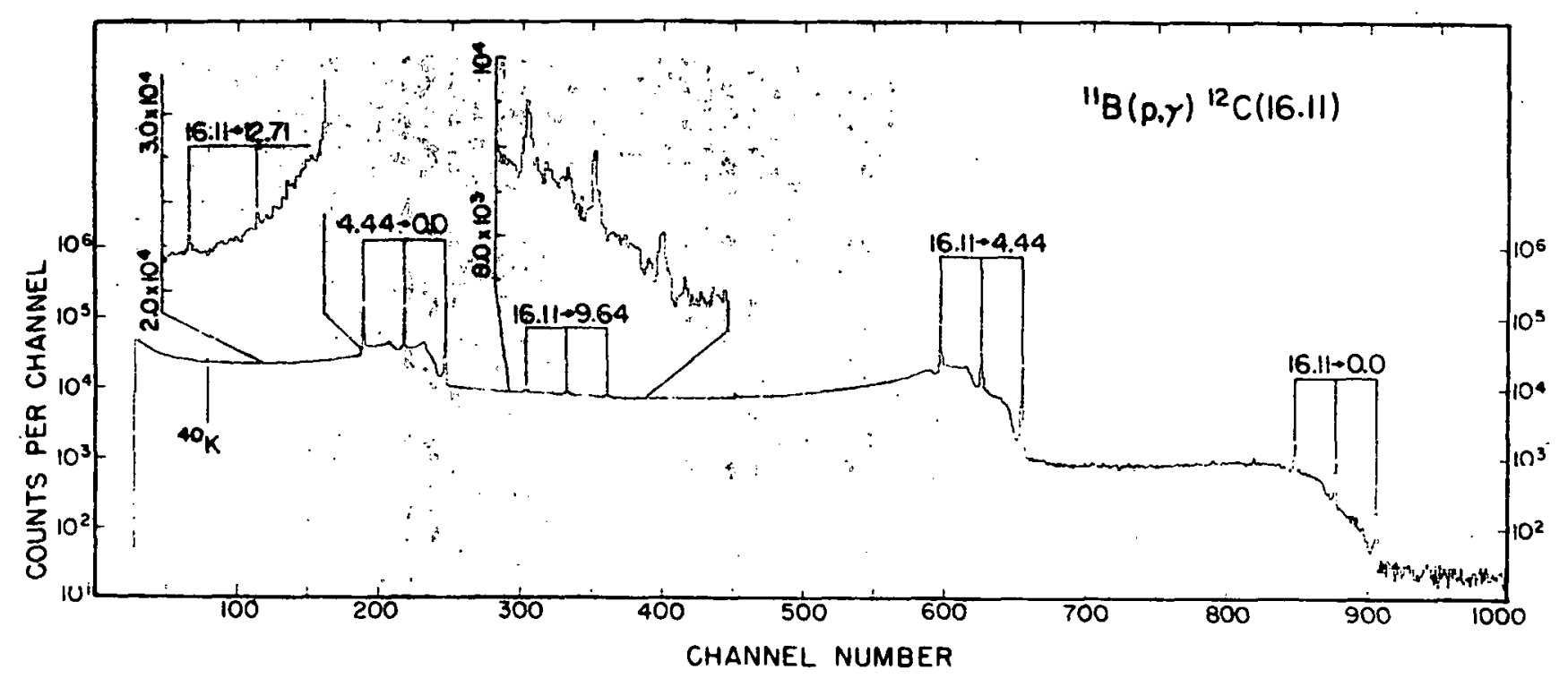

Fig. 8.2-1. Spectrun of $\gamma$ rays from the $163 \mathrm{keV}{ }^{11} \mathrm{~B}(\mathrm{p}, \gamma)$ resonance observed in a $15 \% \mathrm{Ge}(\mathrm{Li})$ detector. The weak "three and four escape peaks" are due to summing of the $16.1 \rightarrow 4.4 \rightarrow 0.0$ cascade.

that as expected the isovector impurity in the $12.7 \rightarrow 0.0$ transition is dominated by an admixture of the $15.1 \mathrm{MeV}$ level into the $12.7 \mathrm{MeV}$ state. This is due in part to the small energy denominator, but even more importantly because the $15.1 \mathrm{MeV}$ level nearly exhausts the isovector MI strength from the ${ }^{12} \mathrm{C}$ ground state. Therefore this transition provides an excellent, nearly modelindependent way to measure the 15.1-12.7 mixing. By fitting the observed ratio $\Gamma_{\gamma}(12.7+0.0) / \Gamma_{\gamma}(15.1+0.0)$ to that calculdted from $C K$ matrix elements we find $B=+0.046 \pm 0.012$ where we have included a $20 \%$ uncertainty in the theoretical isoscalar rate (see Fig. 8.2-2). The second solution to the quadratic equations, wilh negatlve $B$, is not consistent with transfer reaction data. The $12.7 \rightarrow 4.4$ transition does not provide a good measure of $\beta$ because the isospin impurity in this transition can have 4 significant components, due to admiritures into $12 \mathrm{C}(12.7)$ of the two lowest $1^{+} \mathrm{T}=1$ levels and admixture into ${ }^{12} \mathrm{C}(4.4)$ of the two lowest $2^{+} T=1$ levels. (The admixtures with large energy demoninators are important because of their large MI matrix elements.)

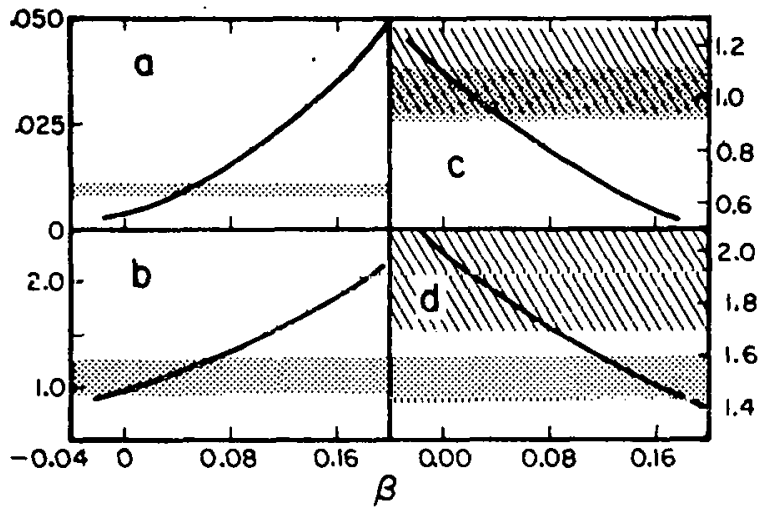

Fig. 8.2-2. Experimental (shaded area) vs calculated (solid line) quantities as a function of $\beta . a) \Gamma_{\gamma_{0}}\left(12.7 / \Gamma_{\gamma_{0}}(15.1) ; b\right.$, c) $s(12.7) / S(15.1)$ from stripping and pickup reopcctively; d) $3\left({ }^{1}{ }_{\mathrm{B}} 0.0\right) / S(15.1)$ from pickup. Hatched areas are from ${ }^{3} c(p, d)$. We assume relative errors of 108 for $\left(d,{ }^{3} \mathrm{He}\right)$ and $(d, t)$ and $15 \%$ for $\left.{ }^{3} \mathrm{He}, d\right)$ and $(p, d)$ spectroscopic factors. 
Table 8.i-1. Comparison of A=12 Observables with Theory

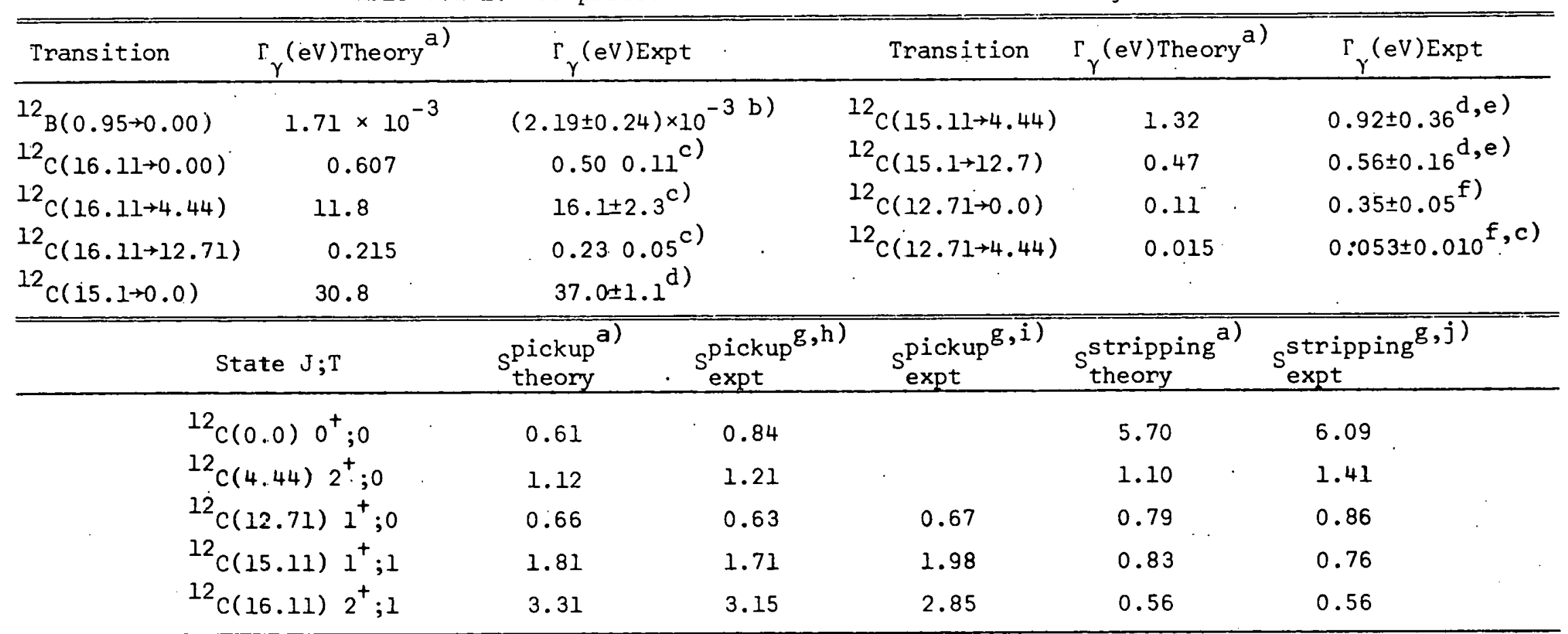

a) Ref. 9

上) J.W. Olness and E.K. Warburzon, Phys. Rev. 166, 1004 (1968).

c) This work.

c) B.J. Chertok et al., Phys. Rev. C 8, 23 (1973).

€) D.E. Alburger and D.H. Wilkinson, Phys. Rev. C 9,384 (1972).

f) Ref. 5 .

E) Normalization: $(S(12.7)+S(15.1)+S(16.1))$
the $\operatorname{Ref.} 12.13 \mathrm{C}(\mathrm{p}, \mathrm{d})$.

i) Ref. 2. $13 \mathrm{c}(\mathrm{d}, \mathrm{t})$.

j) P.D. Miller etal., Nucl. Phys. Al36, 229 (1969). ${ }^{11} \mathrm{~B}\left({ }^{3} \mathrm{He}, \mathrm{d}\right)$. 
We now examine the ${ }^{1 l_{B}}\left({ }^{3} \mathrm{He}, d\right)$ proton stripping and ${ }^{13} \mathrm{C}(\mathrm{d}, t)$ neutron pickup spectroscopic factors for the 12.7 and $15.1 \mathrm{MeV}$ levels. We expect these to provide an independent although less reliable measure of the 15.1-12.7 isospin mixing. The two state mixing approximation is valid in this case because the $C K$ stripping and pickup amplitudes are much larger for the 12.7 and $15.1 \mathrm{MeV}$ levels than for any other $J=1$ states. Isospin mixing induced by Coulomb forces causes the lower lying member of an analog-antianalog doublet to have an enhanced parentage to $l l_{B}+p$ compared to ${ }^{1 l_{C}}+n$, while the upper member of the doublet is preferentially $1 l_{C}+n$. Hence proton stripping should preferentially populate the lower member of the doublet, and neutron pickup the higher member. This phenomenon is well known from the celebrated case of the $16 \mathrm{MeV}$ states in ${ }^{8} \mathrm{Be}$ and follows from the fact that a proton particle-hole excitation has a lower energy than the corresponding neutron particle-hole excitation due to the Coulomb pairing energy.

In Table 8.2-1 we tabulate relative spectroscopic factors in $A=12$. It can be seen that $C K$ give a good account of those quantities not affected by 15.1-12.7 mixing. The experimental ratio $R=\sigma(15.1) / \sigma(12.7)$ is slightly greater in $13 c(d, t)$ and slightly smaller in $11_{B}\left({ }^{3} \mathrm{He}, d\right)$ than the corresponding ratios calculated by CK, which is independent evidence for isospin mixing in the $1^{+}$doublet. The values of the mixing parameters $\beta$ required to account for the pickup and stripping ratios are $B=+0.020 \pm 0.025$ and $B=+0.038_{-}^{+.030}$ respectively (see Fig. $8.2-2$ ). These values are in gratifying agreement both in magnitude and sign with that. extracted from the MI decays of the $12.7 \mathrm{MeV}$ level. Is there enough "slop" in the CK calculation to reconcile the experimental MI widths and spectroscopic factor ratios with the BBCG value of $\beta=0.11$ ? We think not. From Fig. 8.2-1 we see that the ratio of predicted to experimental values of $\Gamma_{\gamma}(12.7 \rightarrow 0) / \Gamma(15.1 \rightarrow 0)$, $R\{13 C(d, t)\}$ and $R\left\{1 I_{B}\left({ }^{3} \mathrm{He} ; d\right)\right\}$ would be $2.29 \pm 0.33,0.69 \pm 0.07$, and $1.32 \pm 0.20$ respectively if $\beta=0.11$. Since these discrepancies are notably greater than those occurring in transitions not sensitive to the isospin mixing, we conclude that $\beta$ must be much smaller than 0.11 .

Let us summarize. Based on a $300 \%$ effect in the relative $\gamma_{0}$ strengths of ${ }^{12} \mathrm{C}(12.7)$ and ${ }^{12} \mathrm{C}(15.1)$ when compared to the charge independent theory, we derive a matrix element $\left\langle 1\left|\mathrm{H}_{\mathrm{CD}}\right| 0\right\rangle=110 \pm 30 \mathrm{keV}$ which is less than half that found by BBCG. Previously reported large values for the isospin mixing matrix element $\left\langle I\left|H_{C D}\right| 0\right\rangle$ were derived from direct reaction studies which displayed $1 \%$ and $30 \%$ anomalies, when compared to charge independent predictions, and in part relied heavily on the DWBA. However all other transfer reaction data is consistent with the matrix element deduced from the $\gamma$-rays. We conclude that, with the possible exception of ${ }^{8} \mathrm{Be}$, there is no evidence from isospin mixing for a sizable $\Delta \mathrm{T}=1$ component of the short-range nuclear force.

We are grateful to $D$. Kurath for kindly sending us the CK matrix elements and to Prof. P.D. Parker for generous assistance in data taking at Caltech.

\footnotetext{
$\therefore \quad$ Supported in part by ERDA (Univ. Wash.), NSF (Caltech), and the A.P. Sloan Foundation (EGA).

$\dagger \quad$ Present address: Department of Physics, California Institute of Technology, Pasadena, CA.
} 
1. F.C. Barker, Nucl. Phys. 83, 418 (1966).

2. W.J. Braithwaite et al., Phys. Rev. Lett. 29, 376 (1972).

3. F.D. Reisman et al., Nucl. Phys. A153, 244 (1970).

4. J.W. Negele, Proc. Int. Conf. Nucl. Structure and Spectroscopy, ed.

H.P. Blok and A.E.L. Dieperink(Scholars Press Amsterdam, 1974) P. 618.

5. F.E. Cecil et al., Phys. Rev. C 9, 798 (1974).

6. D.P. Balamuth et al., Phys. Rev. C 10,975 (1974).

7. A. van der Woude et al., Phys. Rev. C 10, 952 (1974).

8. J. Spuller et al., Nucl. Phys. A248, 276 (1975).

9. S. Cohen and D. Kurath, Nucl. Phys. 73, I (1965); Nucl. Phys. A101, 1 (1967). .

10. R.E. Marrs et al., Phys. Revi. Lett. 35, 202 (1975).

11. B.P. Singh and H.C. Evans, Nucl. Instrum. Methods 97, 475 (1971).

12. H. Taketani et al., Phys. Lett. 27B, 625 (1968).

8.3 Charged Particle and $\gamma$ Ray Decays of the Lowest $\mathrm{T}=2$ States of ${ }^{24} \mathrm{Mg},{ }^{28} \mathrm{Si}$, ${ }^{32} \mathrm{~S}$ and ${ }^{44} \mathrm{Ti}$

E.G. Adelberger, S.J. Freedman ${ }^{\dagger}$, A.B. McDonald ${ }^{\dagger+}$, M. Oothoudt ${ }^{\dagger}$, R.G.H. Robertsont, and F. Zutavernt

- We have used the AVF cyclotron and QDDD spectrograph at Princeton University to study the decays of the lowest $T=2$ states in the $A=4 n$ nuclei. $A 41.6$ $\mathrm{MeV}$ proton beam bombarded thin $\left(2100 \mu \mathrm{gm} / \mathrm{cm}^{2}\right)$ targets of $26 \mathrm{Mg},{ }^{30} \mathrm{Si},{ }^{34} \mathrm{~S}$ and ${ }^{46} \mathrm{Ti}$, and tritons populating the $\mathrm{T}=2$ states of the residual nuclei were detected with large solid angle $(\Delta \Omega=14 \mathrm{msr})$ and good resolution $(\Delta \mathrm{E} \sim 25 \mathrm{keV})$ at $\theta_{\mathrm{L}}=$ $22.5^{\circ}$ in the QDDD. Decay particles were detected in a solid state counter telescope at $\theta_{\mathrm{L}}=-90^{\circ}$ or $\theta_{\mathrm{L}}=-120^{\circ}$. For the ${ }^{24} \mathrm{Mg},{ }^{28} \mathrm{Si}$ and ${ }^{44} \mathrm{Ti}$ measurements the decay telescope consisted of a $50 \mu \Delta \mathrm{E}$ and a $1000 \mu \mathrm{E}^{\prime}$ counter. For the $32 \mathrm{~S}$ measurement we employed a 3 counter telescope of $16 \mu, 50 \mu$ and $1000 \mu$ counters. Decay $\gamma$-rays were detected in a $5 " \times 4^{\prime \prime} \mathrm{NaI}$ detector. Reasonable singles counting rates were obtained in the NaI detector since the proton beam was dumped in a well shielded location $210 \mathrm{~m}$ away from the target.

Tritons were identified in the QDDD by hard-wired windows on the pulse heights in a thin gas $\Delta E$ counter and a thick plastic scintillator. A transverse electric field located between two of the dipole elements, was used to improve the discrimination against $\mathrm{p}^{\prime} \mathrm{s}$, d's, etc. Event-mode recording on magnetic tape was used to obtain 6 parameter data: Triton rigidity (obtained from a hard-wired division of signals from the resistive wire gas proportional counters), signals in the $\triangle E, E^{\prime}$ and $\gamma$ ray counters, and TAC's giving charged particle-triton coincidences $\gamma$-ray triton coincidences. For the $32 \mathrm{~s}$ measurement the $\gamma$-ray detector was removed and signals from the second $\Delta E$ detector were stored instead. Triton singles and coincidence data were accumulated simultaneously in the same ADC in order to remove most sources of systematic errors. The coincidence efficiency of our apparatus was determined by studying the isotropic decays of the $8.92 \mathrm{MeV}$ $1 / 2^{-}$level of $13_{\mathrm{N}}$ populated in $14 \mathrm{~N}(\mathrm{p}, \mathrm{d})$. Charged particle efficiency was determined from the $\mathrm{P}_{0}$ and $\mathrm{P}_{1}$ decays to $12 \mathrm{C}(0.0)$ and ${ }^{12} \mathrm{C}(4.4)$. Gamma ray efficiency was measured using the $4.4 \mathrm{MeV} \gamma$-rays following the $\mathrm{Pl}_{1}$ decays. Analysis of the data is in progress. Preliminary results reveal some disagreement with earlier 
work on ${ }^{44} \mathrm{Ti}^{2}$ For ${ }^{44} \mathrm{Ti}$ we obtain an $\alpha$ branch of $\approx 20 \%$ with the rest of the decays being via $\gamma$-rays.

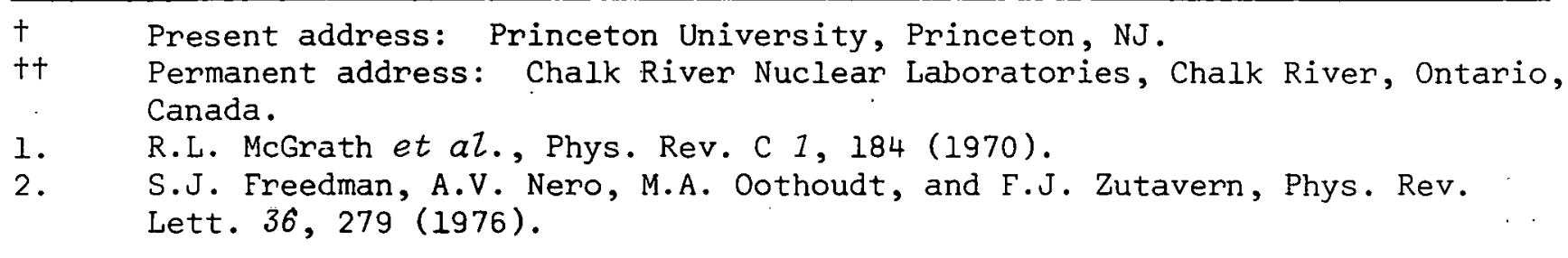

8.4 Charged Particle and Neutron Decays of the Lowest $\mathrm{T}=2$ States in ${ }^{8} \mathrm{Be},{ }^{12} \mathrm{C}$ and ${ }^{16} 0$

E.G. Adelberger, S.J. Freedman ${ }^{\dagger}$, A.B. McDonald ${ }^{\dagger \dagger}$, M. Oothoudt ${ }^{\dagger}$, R.G.H. Bebenteon', and F. Zutavenn'

The decays of the lowest $\mathrm{T}=2$ states of ${ }^{8} \mathrm{Be},{ }^{12} \mathrm{C}$ and ${ }^{16} \mathrm{O}$ were studied using the apparatus discussed in Sec. 8.3 above. The work on $8_{\mathrm{Be}}$ and ${ }^{12} \mathrm{C}$ represents a continuation of work discussed in last year's Annual Report.l

$16 \mathrm{~A} \mathrm{W0} 3$ target enriched in ${ }^{18} 0$ was bombarded with $41.6 \mathrm{MeV}$ protons for the ${ }^{16} \mathrm{O}$ measurement. $10 \mathrm{BeO}$ and $14 \mathrm{C}$ targets were bombarded at $45.9 \mathrm{MeV}$ for the ${ }^{8} \mathrm{Be}$ and $12 \mathrm{C}$ experiments. 160 data was taken with decay telescopes consisting of $50 \mu+$ $1000 \mu$ detectors and $16 \mu+50 \mu+1000 \mu$ detectors. The ${ }^{8} \mathrm{Be}$ and ${ }^{12} \mathrm{C}$ experiments were done with $15 \mu+35 \mu+1000 \mu$ detectors. A particular effort was made to detect branches involving low energy particles such as the deuteron channel. Neutron decays were detected in a $2 " \times 5^{\prime \prime}$ plastic scintillator. Analysis of the data is in progress.

$+\quad$ Present address: Princeton University, Princeton, NJ.

tw Hermanent address: Chalk Kiver Nuclear Laboratories, Chalk Kiver, Ontario, Canada.

1. Nuclear Physics Laboratory Annual Report, University of Washington (1975), p. 55.

\subsection{Lifetimes of Isomeric States in ${ }^{174} \mathrm{Hf}$}

H. Bohn, Yod,Chan, H. Ejiri ${ }^{\dagger}$, and R. Sielemann

While there is much information available about the ground state and $B-$ vibrational state rotational band structure in $174 \mathrm{Hf}$ up to very high spins (22 $\pi$ ) mainly obtained via $(\alpha, x n \gamma)^{\prime} \perp$ and $(H I, x n y)^{2}$ reactions, only little precise information exists about the properties of quasiparticle states in this nucleus. Within the even-even $H f$ isotopes up to now the best studied nucleus in this respect is $176_{\mathrm{Hf}}$, where three isomeric quasiparticle states $\left(\mathrm{K}^{\pi}=6^{+}, \mathrm{K}^{\pi}=8^{-}, \mathrm{K}^{\pi}=14^{-}\right)$ with long halflives $(9.5 \mathrm{\mu s}, 9.8 \mathrm{\mu s}, 401 \mathrm{\mu s})$ are found. ${ }^{3}$

We started an investigation of isomeric states in ${ }^{174} \mathrm{Hf}$ using a pulsed beam technique. We used the $175 \mathrm{Lu}(\mathrm{p}, 2 \mathrm{n})$ reaction at bombarding energies of $12-$, 
14-, and 16-MeV. The proton beam was chopped into pulses of about 100 ns width and 8 us repetition time. The $\gamma$-rays were detected with a coaxial, $53 \mathrm{~cm}^{3} \mathrm{Ge}(\mathrm{Li})$ detector as well as with a small planar Ge(Li) detector. The latter was used to: cover the low energy $\gamma$-ray region ( $\leq 100 \mathrm{keV}$ ). Data were taken in a two-dimensional mode ( $\gamma$-ray energy vs time since the preceding proton beam burst) and stored on magnetic tape. The prompt events were scaled down by a factor of 10 or 100 in order to reduce the deadtime of the ADC's of the online computer. The time information was obtained via a time-to-amplitude converter, which was started by the constant fraction signal of the $\mathrm{Ge}(\mathrm{Li})$ detector and stopped by an appropriate signal from the chopper.

In the prompt $\gamma$-ray energy spectrum obtained at $16 \mathrm{MeV}$ bombarding energy the ground state rotational band in $174_{\mathrm{Hf}}$ is observed up to $\mathrm{J} \pi=12^{+}$. In the delayed part several isomeric $\gamma$-transitions in a time range between 100 ns and $8 \mathrm{\mu s}$ are observed and are listed in Table 8.5-1 together with halflives from a preliminary analysis at $16 \mathrm{MeV}$ bombarding energy.

Table 8.5-1. Measured Halflives for Different $\gamma$-Ray Transitions in ${ }^{174} \mathrm{Hf}$

\begin{tabular}{ccr}
$\begin{array}{c}E_{\gamma} \\
(\mathrm{MeV})\end{array}$ & $\begin{array}{c}\text { Observed Halflife } \\
(\mu \mathrm{s})\end{array}$ & $\begin{array}{l}\text { Associated } \\
\text { Transition } \\
\text { in } 174 \mathrm{Hf}\end{array}$ \\
\hline .188 & 2.1 & $? \rightarrow 6^{+1}$ \\
.353 & 1.4 & $(8) \rightarrow 6^{++}$ \\
.941 & $.18, \approx 2$ & $6^{++} \rightarrow 6^{+}$ \\
1.252 & $.18, \approx 2$ & $6^{++} \rightarrow 4^{+}$
\end{tabular}

Experimental time spectra are shown in Fig. 8.5-1 together with a partial. level scheme ${ }^{4}$ of ${ }^{174} \mathrm{Hf}$.

The $.941 . \mathrm{MeV}$ and $1.252 \mathrm{MeV} \gamma$-raye are known to depopulate a $\mathrm{J}^{\pi}-6^{+}$isomeric state at $1.549 \mathrm{MeV}$ in $174 \mathrm{Hf}$. A former lifetime determination $\mathrm{g}$. $2.1 \mu \mathrm{s}^{5}$ for this state. This is in disagreement with our data, winich show together with the information from the data obtained at $12 \mathrm{MeV}$ and $14 \mathrm{MeV}$ bombarding energy that clearly the shorter halflife of $T_{1 / 2} \approx 180 \mathrm{~ns}$ must be assigned to this state. At $12 \mathrm{MeV}$ bombarding energy this halflife almost exclusively shows up whereas at $14 \mathrm{MeV}$ and $16 \mathrm{MeV}$ an increasing component of about 2 us is noticeable. This shorter halflife is represented in Fig. 8.5-1 by a plot of the time distribution on an expanded scale of the $6^{+} \rightarrow 4^{+}$transition, which carries the same delayed information as the $6^{+1} \rightarrow 6^{+}$or $6^{+1} \rightarrow 4^{+}$transitions.

The $0.353 \mathrm{MeV} \gamma$-ray line with $\mathrm{T}_{1 / 2}=1.4$ us shows no prompt component even at $16 \mathrm{MeV}$ bombarding energy, indicating that this line directly depopulates the isomeric state. This would then be in agreement with a former tentative assignment ${ }^{4}$ which placed this state at $1.902 \mathrm{MeV}$ excitation energy with $\mathrm{T}_{1 / 2}>200 \mathrm{~ns}$. 
The $0.188 \mathrm{MeV} \gamma$-ray line with $\mathrm{T}_{1 / 2}=2.1 \mathrm{us}$ has a strong prompt component. A transition with this energy is believed to originate from the first excited state $\left(7^{+}\right)$of the band built on the $6^{+}$isomeric state at $1.549 \mathrm{MeV} .4$ The impression of Ref. 4 that the 0.188 $\mathrm{MeV} \gamma$-ray should carry the same halflife information as the $0.353 \mathrm{MeV} \gamma$-ray originating from the isomeric state at 1.902 $\mathrm{MeV}$ is not confirmed by our data. We clearly observe different halflives for both transitions (compare Fig. 8.5-1 a and $\mathrm{b}$ ) and we can not find a $0.165 \mathrm{MeV}$ transition which should connect the $1.902 \mathrm{MeV}$ state with the $7^{+}$state. ${ }^{4}$

If one assumes that both the 0.188 and $0.353 \mathrm{MeV} \gamma$-ray transitions contribute to the population of the isomeric $6^{+}$state at $1.549 \mathrm{MeV}$ as given in Ref. 4 then three different halflives $\left(\mathrm{T}_{1 / 2}=\right.$ $180 \mathrm{~ns}, \mathrm{~T}_{1 / 2}=1.4 \mu \mathrm{s}, \mathrm{T}_{1 / 2}=2.1 \mu \mathrm{s}$ ) should be apparent in the time spectra of the $0.941 \mathrm{MeV}$ and $1.252 \mathrm{MeV} \gamma$-ray transitions depopulating this state. Only the short lived component can be distinguished (see Fig. 8.5-le) from a longer lived component of about $2 \mu \mathrm{s}$ as mentioned above. For the $16 \mathrm{MeV}$ data presently under analysis the statistics are not good enough to decide whether or not the longer lived component can be decomposed into different halflives.

The further analysis of a number of weaker $\gamma$-ray lines also observed in the delayed spectra may help to locate the two longer lived isomeric states.

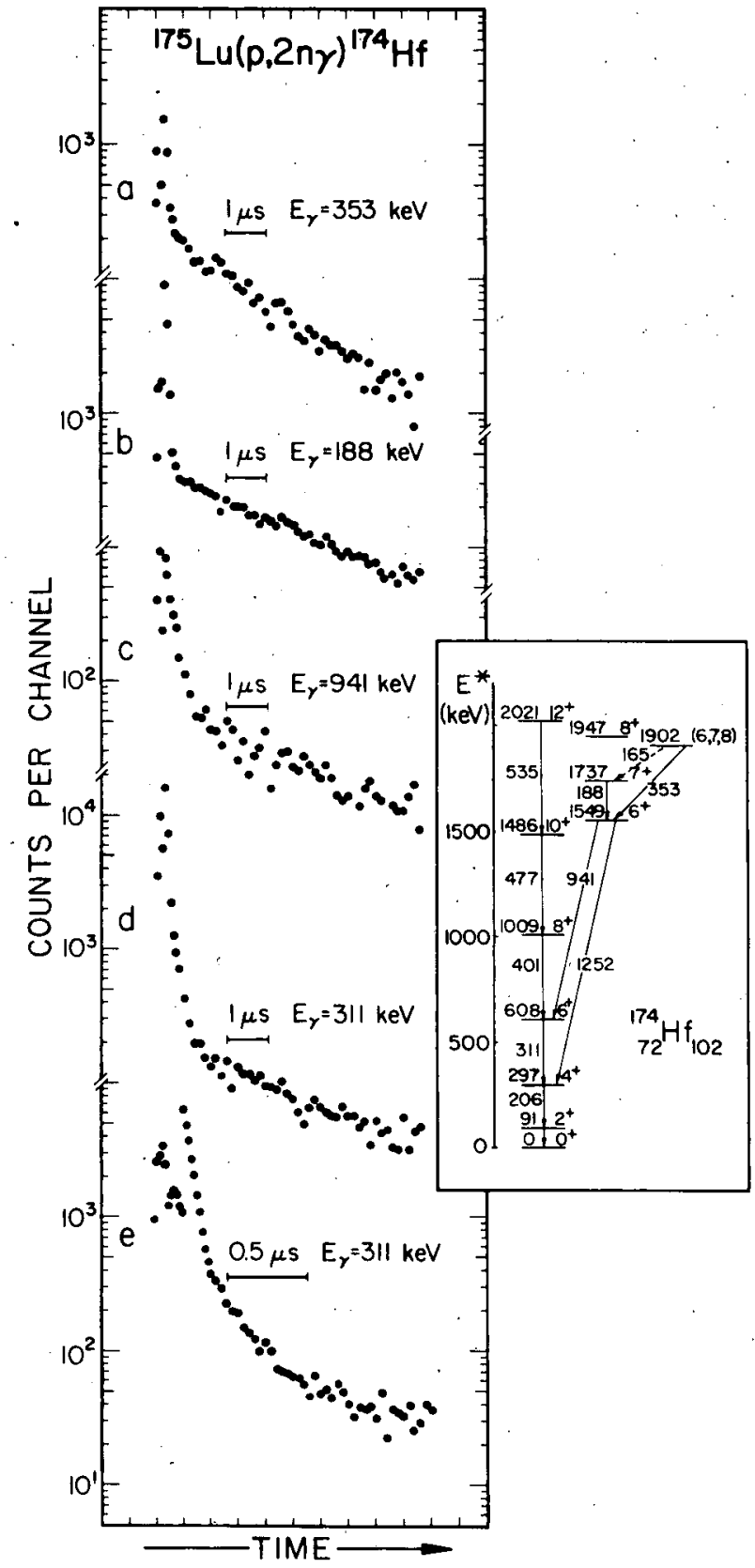

Fig. 8.5-1. Time distributions of delayed $\gamma$-ray transitions in $174 \mathrm{Hf}$ following the reaction $175 \mathrm{Lu}(\mathrm{p}, 2 \mathrm{n})$ with a pulsed proton beam at $16 \mathrm{MeV}$ bombarding energy. The inset shows the related part of the ${ }^{174} \mathrm{Hf}$ level scheme. 
$\dagger$ Permanent address: Department of Physics, Osaka University, Toyonaka, Osaka, Japan.

1. H. Ejiri and G.B. Hagemann, Nucl. Phys. A161, 449 (1971).

2. H. Bohn, T.v. Egidy, T. Faestermann, F.v. Feilitzsch, P. Kienle, H.J. Körner, and J.P. Schiffer, Annual Report, Munich (1974), p. 47.

3. T.L. Khoo, F.M. Bernthal, R.A. Warner, G.F. Bertsch, and G. Hamilton, Phys. Rev. Lett. 35, 1256 (1975).

4. I. Rezanka, H. Ryde, and S. Hultberg, Physica Scripta 8, 239 (1973).

5. H. Ejiri, S.M. Ferguson, R. Heffner, and M. Wieman, Proc. of Int. Conf. on Radioactivity in Nucl. Spectr., Nashville, Tennessee (1969).

8.6 Study of Low Lying Dipole States in Pb Isotopes via Analog Resonant $\left(p, p^{\prime} \gamma\right)$ 三

D. Chiang, J.G. Cramer, and P.A. Dickey

207 Elastic photon scattering cross sections ${ }^{1}$ below neutron threshold in ${ }^{206} \mathrm{~Pb}$, ${ }^{207} \mathrm{~Pb}$, and ${ }^{208} \mathrm{~Pb}$ exhibit a striking similarity in the concentration of El transition strength between 4 and $8 \mathrm{MeV}$. Shell model calculations 2 have demonstrated the sensitivity of dipole strength in and below the giant resonance to the amplitudes and phases of configurations in the nuclear levels. We have been studying these low lying dipole states via inelastic proton scattering through isobaric analog resonances (IAR) in an attempt to see how the coupling of $\mathrm{P}_{1 / 2}$ neutron holes to the ${ }^{208} \mathrm{~Pb}$ core affects the configuration purity of core excitations.

Figure 8.6-1 illustrates schematically the technique of IAR spectroscopy for a $208 \mathrm{~Pb}$ target. Proton inelastic scattering through analog resonances excites neutron particle-hole states with single, selectable particle configurations. If further one measures in coincidence strong gamma decays to the target ground state, the $1^{-}$states are preferentially selected. ${ }^{3}$ The spin of the neutron particle is the same as that of the IAR; the spin of the hole must be that of the outgoing proton.

The proton beam from the University of Washington tandem was used to excite the IAR in $207 \mathrm{Bi},{ }^{208} \mathrm{Bi}$, and $209 \mathrm{Bi}$ by bombardment of $0.5-1.0 \mathrm{mg} / \mathrm{cm}^{2}$ targets of $206 \mathrm{~Pb}, 207 \mathrm{~Pb}$, and $208 \mathrm{~Pb}$ respectively. Incident energies corresponded to the $\mathrm{d}_{3 / 2}, \mathrm{~s}_{1 / 2}$, and $\mathrm{d}_{5 / 2}$ resonances for each target; off resonance data were accumulated to estimate the direct background. Protons were detected at $90^{\circ}$ in a $3 \mathrm{~mm}$ thick Si(Li) detector, cooled and equipped with a sweeping magnet; the resolution thus obtained was $34 \mathrm{keV}$ FWHM. Gamma rays were detected in the NPL 10" $\times 10^{\prime \prime} \mathrm{NaI}$ spectrometer, collimated to an acceptance angle of $\pm 7.5^{\circ}$. Coincidences were recorded with the $\mathrm{NaI}$ placed at $55^{\circ}, 75^{\circ}, 90^{\circ}, 105^{\circ}$, and $125^{\circ}$ in the reaction plane, and at $90^{\circ}$ out of plane.

The simultaneous singles-coincidence data handling programs SCOSINDHAPSCOSINEAP were used to event record and subsequently playback the coincidence data. In addition, a proton singles bpectrum was accumulated through the same $A D C$ which handled proton coincidences. On playback, two dimensional plots of 


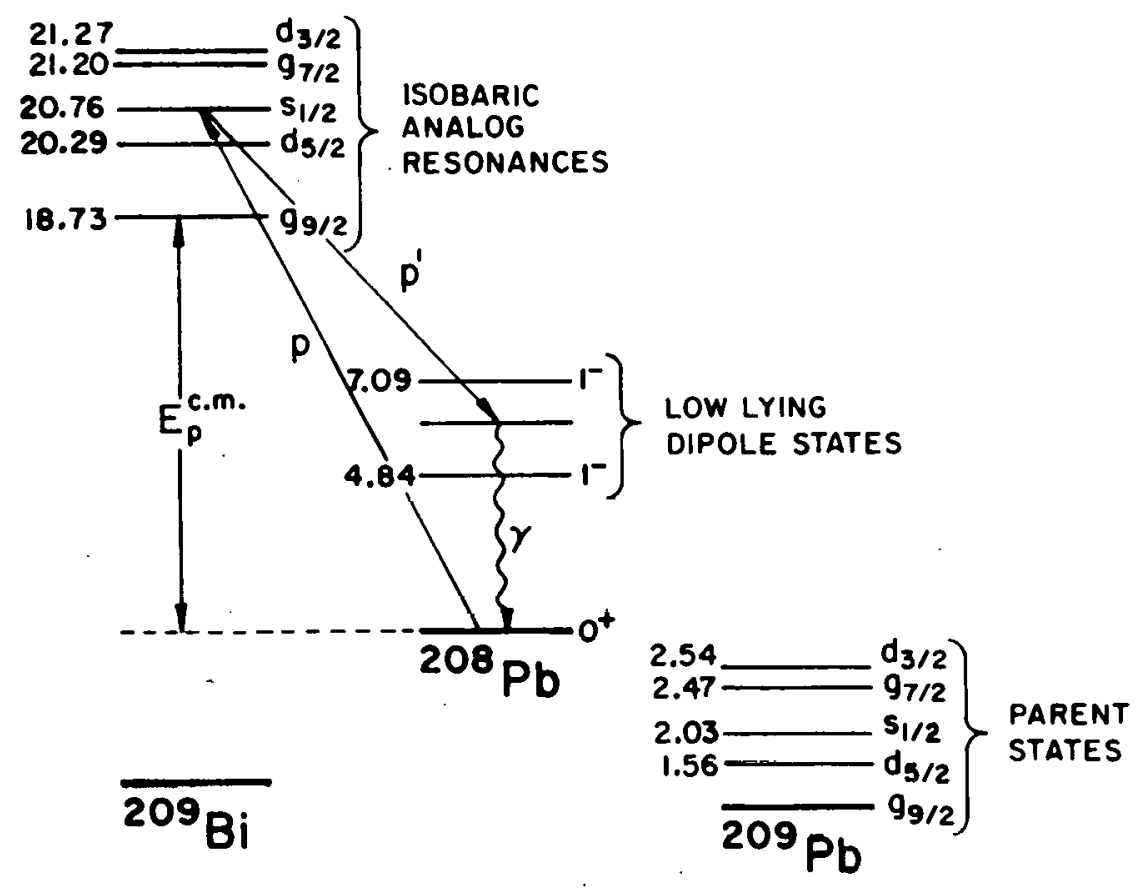

Fig. 8.6-1. Partial level schemes for states of interest in $208 \mathrm{~Pb}\left(\mathrm{p}, \mathrm{P}^{\prime} \gamma\right)$. Arrows indicate excitation of a $1^{-}$state in $208 \mathrm{~Pb}$ through the $s_{1 / 2}$ IAR. in $209_{\mathrm{Bi}}$.

proton energy vs gamma energy were generated. Coincident events corresponding to gammas feeding the ground state of $\mathrm{Pb}$ appear along straight lines in these plots. Sums along these kinematic loci were projected back onto the proton axis to jield coincidence spectra.

The absolute efficiency of the NaI detector was measured by using the ${ }^{12} \mathrm{C}\left(\mathrm{p}, \mathrm{p}^{\prime} \mathrm{r}_{\mathrm{O}}\right)$ reaction at $\mathrm{E}_{\mathrm{p}}=15 \mathrm{MeV}$ to produce coincidences between protons and 4.43 MeV gammas and a simultaneous protonsingles spectrum. Corrections were made for the $\mathrm{p}-\gamma$ correlation.

$$
\text { Reeulte -- }{ }^{208} \mathrm{~Pb}
$$

Although it is usually assumed that the 1 states in ${ }^{2} \mathrm{O} \mathrm{Pb}$ have a $100 \%$. gamma decay branch to the ground state, an accurate measurement has never been made. For this reason, and also for the purpose of checking the resonant coincident yields against those obtained in a previous ${ }^{208} \mathrm{~Pb}\left(\mathrm{p}, \mathrm{p}^{\prime} \mathrm{ro}\right.$ ) experiment at Seattle, ${ }^{3}$ we decided to begin our study with $208 \mathrm{~Pb}$. Detection of the inelastically scattered protons at a single angle (total cross sections were measured in Ref. 3) allows coherent interference between amplitudes for different outgoing proton spins, and could significantly alter the resonance yields in this experiment.

208 Figure 8.6-2a compares the on and off resonance coincidence spectra in $\mathrm{Pb}$ to the elastic photon scattering cross section data from Illinois. 1 The $(\gamma, \gamma)$ cross section is given by 

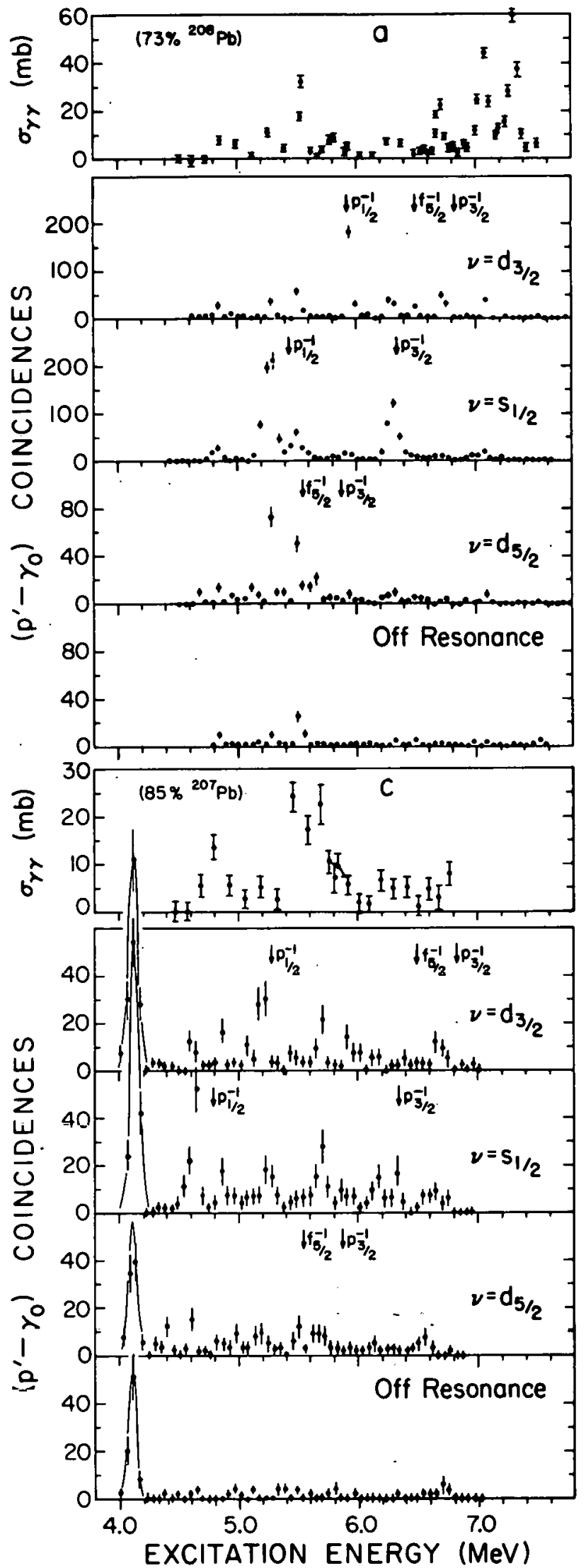

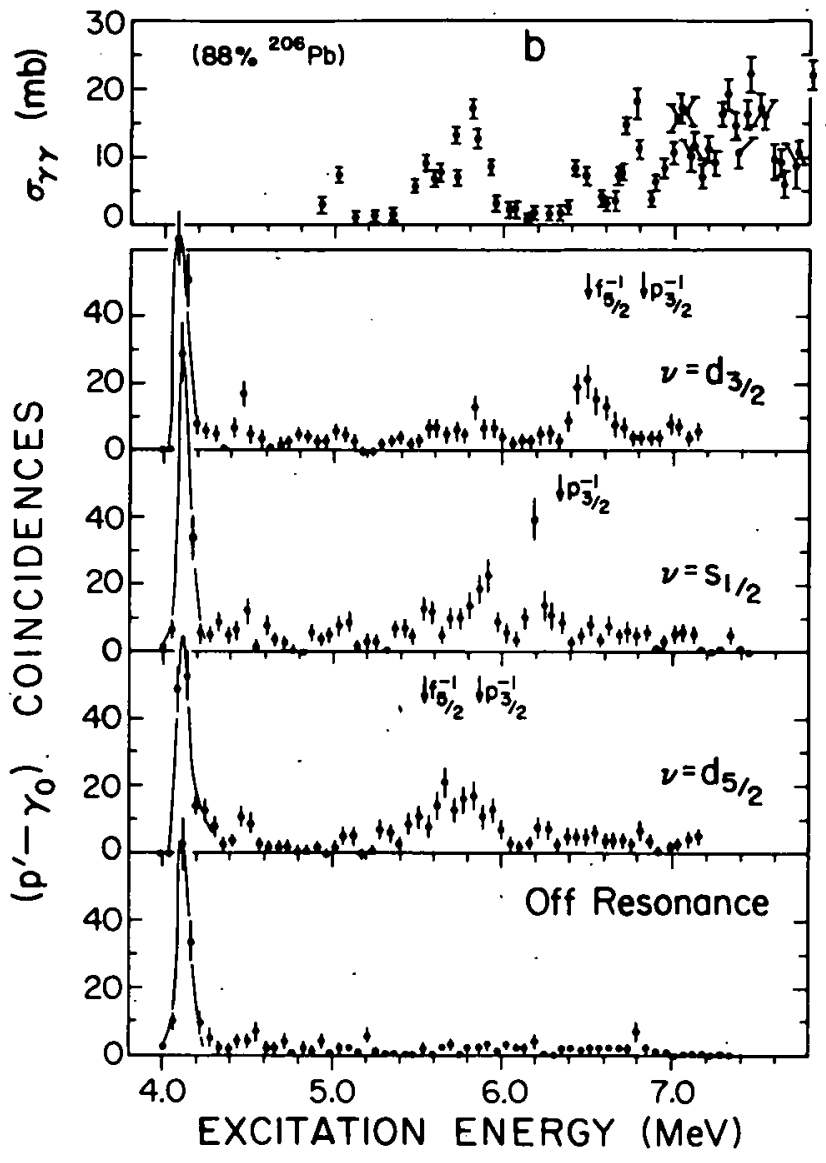

Fig. 8.6-2. Elastic gamma ray cross sections (Ref. I) and coincidence yields obtained in the present experiment.

a) Results from ${ }^{208} \mathrm{~Pb}$. Data for the $s_{I / 2}$ IAR were taken with poorer resolution than the others shown. Arrows indicate energies of unperturbed particle-hole excitations.

b) Results for $206 \mathrm{~Pb}$. The $\mathrm{Pl} / 2$ particle-hole energies are not shown since they are expected to be weak in $206 \mathrm{~Pb}$.

c) Results for $207 \mathrm{~Pb}$. The $\mathrm{p}_{1 / 2}^{-1}$ particle-hole energies have been shifted down in excitation by the difference in photoneutron thresholds $\left(208_{\mathrm{Bn}}-207_{\mathrm{Bn}}\right)$ to estimate the pairing effect. 


$$
\sigma_{\gamma \gamma}(E)=3 \pi \lambda^{2} \frac{1}{D} \frac{\Gamma_{0}^{2}}{\Gamma}
$$

where $\Gamma_{0}$ and $\Gamma$ are the average ground state radiative width and total width respectively of the $1^{-}$levels within the finite beam energy spread and $D$ is the average $1^{-}$level spacing. The quantity, $\Gamma_{0} / D$, defined as the gamma ray strength function, is a measure of dipole transition strength. In contrast, the total $\left(\mathrm{p}, \mathrm{P}^{\prime} \mathrm{Y}_{0}\right)$ coincidence yield is sensitive to the parentage coefficients $\mathrm{a} J j$ for a particle of spin $J$ and a hole of spin $j$ in the state with spin $I$. The total cross section can be written

$$
\sigma_{p p^{\prime}}=\frac{2 T+1}{2} \pi \lambda^{2} \frac{\Gamma_{P_{J}}}{\Gamma_{J}^{2}} \sum_{j}\left|a_{J_{j}}^{I}\right|^{2} \Gamma_{j}^{S P}
$$

where $\Gamma_{J}$ is the total width of the IAR of spin $J$,

$\Gamma_{p}^{J}$ is the proton partial width of the IAR,

and $\quad \Gamma_{j}^{S P}$ is the single particle width for emission of a proton of $\operatorname{spin} j$.

Thus large peaks in $\sigma_{\gamma \gamma}$ indicate concentrations of dipole transition strength, whereas a large resonant $\mathrm{p}, \mathrm{P}^{\prime}$ yield on one IAR results from a dominant particle parentage coefficient.

All of the states appearing in this data have been seen before; however, we have now measured ground state branches for six of these states to be $100 \%$ within $15 \%$ error. (See Table 8.6-l. States not listed were not clearly resolved in proton singles.) Several states show strongly resonant behavior indicative of rather pure particle configurations. Of these, the states at $5.28 \mathrm{MeV}$ (s $1 / 2 \mathrm{par}-$ ticle) and $5.94 \mathrm{MeV}\left(\mathrm{d}_{3 / 2}\right.$. particle) are strongly excited in $207 \mathrm{~Pb}(\mathrm{~d}, \mathrm{p})^{4}$ and thus are predominantly $\mathrm{Pl} / 2$ hole states. The state at $6.3 \mathrm{MeV}$ is largely $\left(\mathrm{s}_{1} / 2 \mathrm{P}_{3} / 2^{-1}\right)$. The strong dipole state at $5.51 \mathrm{MeV}$ exhibits a mixed particle character, whereas the more pure states have comparatively little dipole strength. The absence of large peaks in the proton yields at 7.09 and $7.33 \mathrm{MeV}$ may be attributed to the fact that these strong dipole states are comprised of proton excitations.

It is interesting to note how well the energies of unperturbed shell model configurations (indicated by arrows in Fig. 8.6-2) coincide with the purest states in ${ }^{208} \mathrm{Ph}$.

$$
\text { Results - }{ }^{206} \mathrm{~Pb}
$$

'I'he Level density in $206_{\mathrm{HD}}$ is so Large that the-structures seen in $(\gamma, \gamma)$ and $\left(p, p^{\prime}\right)$ represent the spreading of configurations over many nuclear levels. Further, $\mathrm{pl}_{1 / 2}$ hole excited configurations are not expected in $206 \mathrm{~Pb}$. The two important $\left(\mathrm{Pl} / 2^{-1}\right)$ states in $208 \mathrm{~Pb}$ are clearly absent from the $206 \mathrm{~Pb}$ spectra in

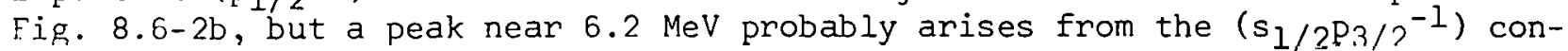
figuration, which apparently has little dipole strength in either nucleus, and is relatively unaffected by the $\left(\mathrm{P} 1 / 2^{-1}\right)$ hole pair. The broad concentration of dipole strength near $5.8 \mathrm{MeV}$ exhibits significant yield on all of the analog resonances, indicative of mixed configurations. This is obviously a complex 
group of states in which the coupling between the $\mathrm{P}_{1 / 2}$ holes and the $5.51 \mathrm{MeV}$ excitation of the $208 \mathrm{~Pb}$ core is not weak.

$$
\text { Results -- }{ }^{207} \mathrm{~Pb}
$$

Weak coupling of a $\mathrm{P}_{1 / 2}$ neutron hole to a $1^{-}$core excitation in ${ }^{208} \mathrm{~Pb}$ would yield a $1 / 2^{+}, 3 / 2^{+}$doublet at the same central energy. If the core excitation has significant $p_{1 / 2^{-1}}$ amplitude, the coupling to another $p_{1 / 2}$ hole would not be weak because of the pairing interaction. In Figure $8.6-2 \mathrm{c}$, we see that the peaks at 4.63 and $5.22 \mathrm{MeV}$ correspond to the two $\mathrm{PI} / 2^{-1}$ states in $208 \mathrm{~Pb}$ shifted down in excitation by $650 \mathrm{keV}$, the energy required to break a $\mathrm{pl} / 2^{-2}$ pair. The more "collective" states at 4.84 and $5.51 \mathrm{MeV}$ in $208 \mathrm{~Pb}$ are not shifted as much in energy, and therefore must feel a much weaker coupling to a $\mathrm{P}_{1 / 2}$ hole because the $\mathrm{Pl} / 2^{-1}$ amplitude in these states is apparently relatively small. The major dipole strength in $207 \mathrm{~Pb}$ at $5.7 \mathrm{MeV}$ resides in collective states which arise from $208 \mathrm{~Pb}(5.51) \otimes \mathrm{P} 1 / 2^{-1}$.

The coincidence yields in ${ }^{207} \mathrm{~Pb}$ are about a factor of four lower than the yields for corresponding states in $208 \mathrm{~Pb}$. Since the energies and width of the IAR in ${ }^{207} \mathrm{~Pb}$ are not well known, it is possible that these data were not collectied at the peak energies of the resonances. The IAR in $208_{\mathrm{Bi}}$ have their parents in the neutron particle-hole states in $208 \mathrm{~Pb}$ which we studied in $208 \mathrm{~Pb}\left(\mathrm{p}, \mathrm{p}^{\prime} \gamma\right)$. Thus we know that spreading of the particle strength among those states may complicate the analog structure. We intend to study this problem in more detail by measuring the excitation functions for the 4.63 and $5.22 \mathrm{MeV}$ states for bombarding energies between 16.5 and $18 \mathrm{MeV}$. Existing inelastic scattering data for lower lying single hole states 5 cannot distinguish the contributions from IAR with different particle configurations.

Table 8.6-1. Ground State Branching Ratios in ${ }^{208} \mathrm{~Pb}$

\begin{tabular}{cc} 
Energy (MeV) & $\frac{\bar{I}_{0} / l^{\prime}}{19}$ \\
\hline 4.84 & $.95 \pm .11$ \\
5.28 & $.97 \pm .07$ \\
5.51 & $.90 \pm .09$ \\
5.63 & $.95 \pm .11$ \\
5.94 & --- \\
6.31 & $.95 \pm .12$ \\
6.49 & --- \\
6.72 & --- \\
7.07 & $.97 \pm .11$ \\
7.09
\end{tabular}

1. R.M. Laszewski and P. Axel, to be published.

2. W.W. True, C.W. Ma, and W.T. Pinkston, Phys. Rev. C 3, 2421 (1971);

M. Harvey and F.C. Khanna, Nucl. Phys. A221., 77 (1974).

3. J.G. Cramer, P. von Brentano, G.W. Phillips, H. Ejiri, S.M. Ferguson, and W.J. Braithwaite, Phys. Rev. Lett. 21, 297 (1968).

4. J. Bardwick and R. Tickle, Phys. Rev. 161, 1217 (1967).

5. N. Stein, Proc. Int. Conf. on Nuclear Isospin, Asilomar (1969), p. 481. 


\section{RADIATIVE CAPTURE MEASUREMENTS AND CALCULATIONS}

9.1 $\frac{V_{\text {The }}{ }^{11}{ }_{B(p, \gamma)^{12} C \text {. Reaction }}}{{ }^{3}}$

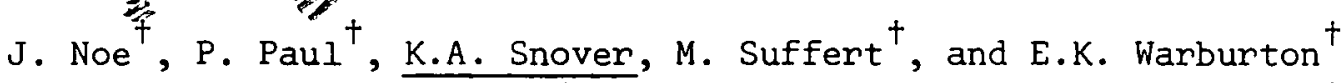

In order to study E2 contributions to radiative capture at energies above the GDR where isovector E2 strength is expected, we have measured the ${ }^{1} 1_{B}\left(p, \gamma_{0}\right) 12 C$ reaction in $1 \mathrm{MeV}$ steps from $E_{\mathrm{p}}=21$ to $29 \mathrm{MeV}$, using the Brookhaven National Laboratory double-MP Tandem Accelerator. Five- and six-point angular distributions were measured between $43^{\circ}$ and $140^{\circ}$ and fit with a Legendre expansion with LMAX $=3$. The results are shown in Fig. 9.1-1, along with previous data at lower energies.

The results are qualitatively similar to previous $15 \mathrm{~N}\left(\mathrm{p}, \gamma_{0}\right) 160$ measurements over a similar energy range; 1 namely, the total cross section falls smoothly with increasing energy, with no sign of a resonance, and the (odd) $a_{1}$ and a coefficients

$$
W(\theta)=A_{n}\left[1+\sum_{i=1}^{3} a_{i} P_{i}(\cos \theta)\right]
$$

rise smoothly, indicating increasing relative importance of $E 2$ at the higher energies. In contrast to $15_{\mathrm{N}}(\mathrm{p}, \gamma) 16_{0}$, the present results show an $a_{3}$ coefficient which continues to grow with energy, with the result that $a$. DSD calculation including direct E2 and direct plus collective EI (solid curves in Fig. 9.1-1) provides a good fit to the data. 4

Collaborators at Brookhaven
National Laboratory, Upton, N.Y.
Nuclear Physics Laboratory Annual
Report, University of Washington
(1975), p. 157.
R.G. Allas et al., Nucl. Phys. 58,
122 (1964).
C. Brassard et al., Phys. Rev, C6,
3. $53(1972) .9$
See Sec. 9.3 of this report.

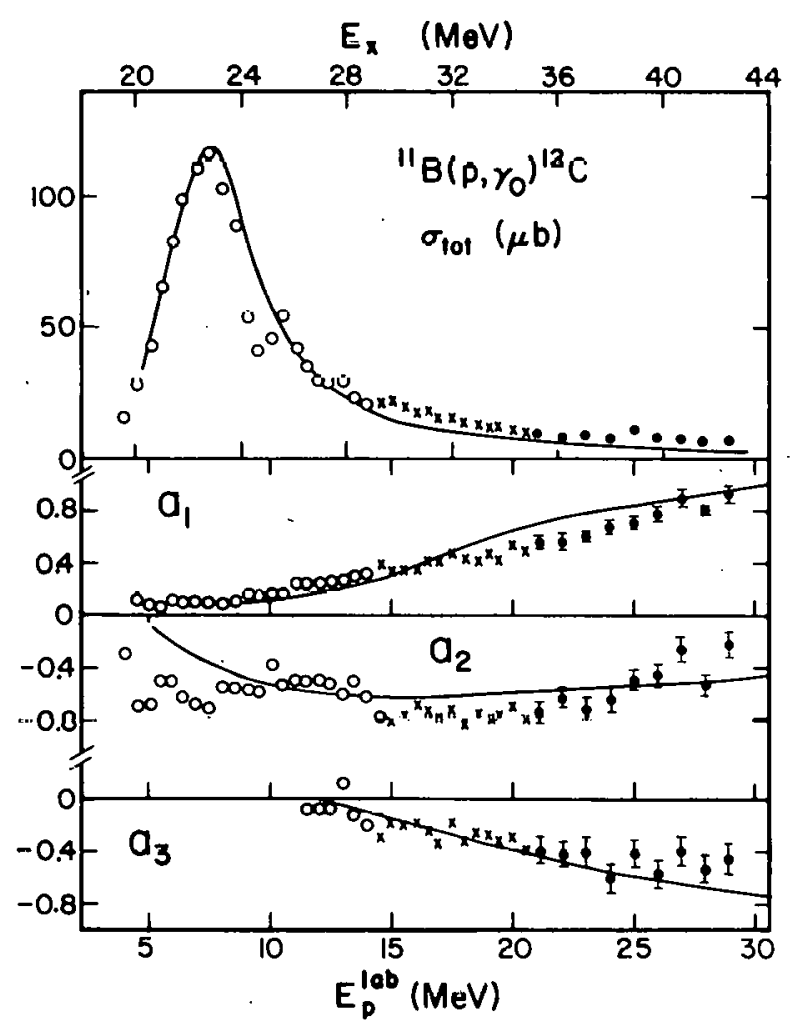

Fig. 9.1-1. The data points represent the meacured total oroes scotion and angular distribution coefficients for the $1 I_{B}\left(p, \gamma_{0}\right)^{12} \mathrm{C}$ reaction: open circles, Ref. 2; crosses, Ref. 3 ; solid points, pressnt work. The solid curves are the rcoult of DSD modcl calculations in cluding direct E2 and direct plus collective El (see Sec. 9.3). 
$9.2 \quad$ The ${ }^{14} \mathrm{C}(\overrightarrow{\mathrm{p}}, \gamma){ }^{15} \mathrm{~N}$ Reaction

J.E. Bussoletti, K. Ebisawa, K.A. Snover, and T.A. Trainor

The first phase of our polarized proton capture measurements on ${ }^{14} \mathrm{C}$, which was described in the 1975 Annual. Report (p. 163), has now been completed. Final results for the measured angular distribution coefficients are shown in Fig. 9.2-1, where

$$
\begin{aligned}
& \sigma(\theta)= \\
& A_{0}\left\{1+\sum_{i=1}^{4}\left[a_{i} P_{i}(\cos \theta)+P b_{i} P_{i}{ }^{1}(\cos \right.\right.
\end{aligned}
$$

$\mathrm{P}=\overrightarrow{\mathrm{P}} \cdot \hat{\mathrm{n}}, \overrightarrow{\mathrm{P}}=$ beam polarization and $\hat{\mathrm{n}} \alpha$ $\vec{k}_{\mathrm{p}} \times \vec{k}_{\gamma}$, the normal to the reaction plane. Final determination of the $a_{i}$ and $b_{i}$ coefficients at each energy was made in an analysis in which $\gamma$-ray yields were normalized to elastic scattering measured in a pair of particle detectors located at $\theta= \pm 160^{\circ}$. Beam polarizations were determined from analyzing power measurements of elastic scattering on ${ }^{12} \mathrm{C}$ and ${ }^{14} \mathrm{C}$ in the target. A typical angular distribution measurement consisted of 3 complete measurements at seven different angles between $43^{\circ}$ and $137^{\circ}$, and statistical consistency between the different measurements was a prerequisite for acceptable data.

The determination of the reaction amplitudes and phases from the measured $a_{i}$ and $b_{i}$ coefficients was done as step 2 in a two-step computer program, the first step being the $a_{i}$ and $b_{i}$ determination. In this way the full error matrix was retained from the first step, including all of the error correlations between the various $a_{i}$ and $b_{i}$. These correlations were found to significantly affect the uncertainties, and in some cases the values, for the reaction amplitudes. The final results are shown in Fig. 9.2-2 for the El amplitudes $s$ and $d$, normalized such that $s^{2}+d^{2}=1$, and the relative

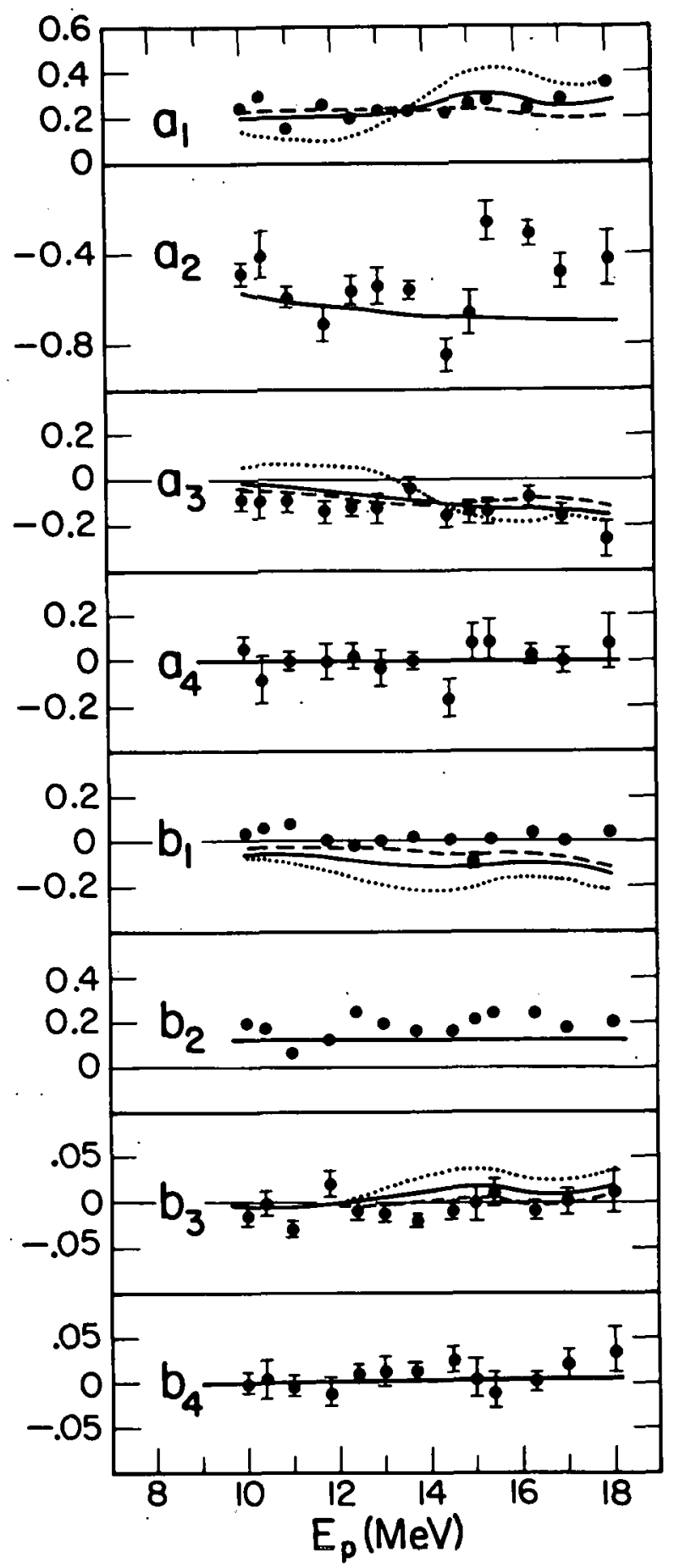

Fig. 9.2-1. Measured and calculated angular distribution coefficients for

${ }^{14} c(p, \gamma)^{15}$. Solid curve: El plus direct

E2; dashed curve: El, direct E2 plus an

E2 IS resonance; dotted curve: El, direct E2 plus an E2 IV resonance. 


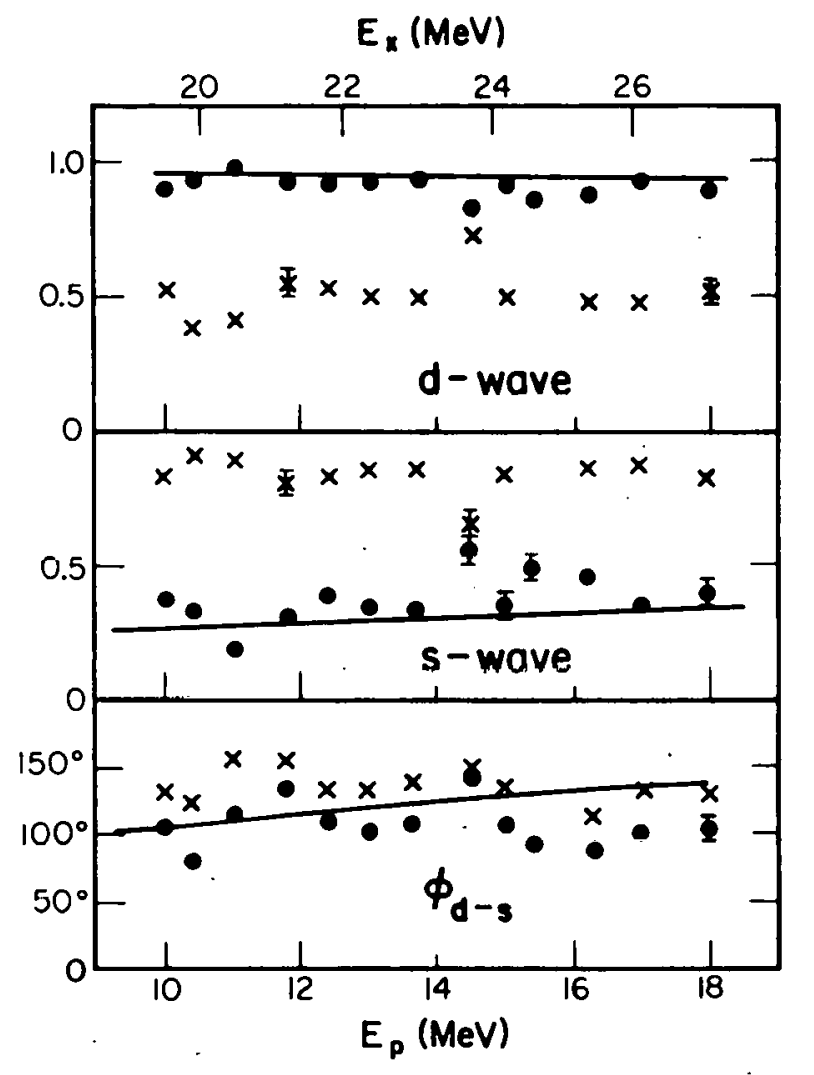

Fig. 9.2-2. The relative El amplitudes and phases. Dots and crosses correspond to the two different solutions at each energy.

phase angle $\phi_{S}-\phi_{d}$, determined from a fit to all $9 \mathrm{a}_{i}$ and $b_{i}$ assuming only $\mathrm{El}$ and E2 (M1 is neglected -- see Sec. 9.3). Two solutions are obtained at each energy, denoted by dots and crosses. The DSD model calculation (see Sec. 9.3) is seen to agree well with the dominant d-wave solution (solid dots). Figure 9.2-3 shows the $\mathrm{E} 2$ amplitude ratio $\mathrm{p} / \mathrm{f}$ and phase differences. In Fig. 9.2-4 we show the measured yo cross section (Ref. 1) and the E2 cross section deduced in this experiment. The solid curves in all cases are a calculation including the effects of direct E2 and direct plus collective El and thus the experiment is seen to agree well with the calculation, indicating little evidence for collective E2 strength in this reaction.

The integral of $\sigma_{E 2}$ from $E_{\mathrm{X}}=19.5$ to $27.0 \mathrm{MeV}$ is $\int \sigma_{E 2}(\gamma, \mathrm{PO}) \mathrm{dE} / \mathrm{E}^{2}=$ $0.48 \pm 0.11 \mathrm{\mu b} / \mathrm{MeV}$ corresponding to $6.8 \pm 1.4 \%$ of the isoscalar (IS) sum rule (the integral of the calculated direct capture is $3.9 \%)$. Thus the $14 \mathrm{C}(\overrightarrow{\mathrm{p}}, \gamma) 15_{\mathrm{N}}$ reaction shows no sign of a collective E2 resonance, although the integral of the observed E2 cross section is somewhat in excess of direct capture. This is in sharp contrast to the situation ${ }^{2}$ in 160 where the integral of the observed $\sigma_{E 2}$ yields $\approx_{35 \%}$ 
of the IS sum and $\sigma_{E 2}$ appears to have a resonance shape. Now over the same energy range $\int \sigma_{E l}\left(\gamma, \mathrm{P}_{0}\right) \mathrm{dE}$ drops in going from 160 to $15 \mathrm{~N}$ but by a much smaller factor ( $14 \%$ vs $7 \%$ of the El sum, respectively). Thus there appears to be a strong difference in the concentration of E2 strength in these two nuclei.

As a check on the absolute cross section normalization, we measured the absolute cross section at $E_{\mathrm{p}}=13.7 \mathrm{MeV}$, $\theta_{\gamma}=90^{\circ}$ for ${ }^{14} \mathrm{C}\left(\mathrm{p}, \gamma_{0}\right)^{15} \mathrm{~N}$. The $\gamma$-ray detector efficiency was determined to a few per cent from a ${ }^{12} C\left(p, \gamma_{0}\right)$ resonance yield measurement, ${ }^{3}$ and the target thickness was determined by a measurement of elastic scattering at $E_{p}=2.20$ and 2.30 $\mathrm{MeV}$, using the absolute cross section measurement reported previously. ${ }^{4}$

Table 9.2-1. Absolute Cross Section for ${ }^{14} \mathrm{C}\left(\mathrm{p}, \gamma_{0}\right){ }^{15} \mathrm{~N}$ at $\mathrm{E}_{\mathrm{p}}=13.7 \mathrm{MeV}$ and $\theta_{\gamma}=90^{\circ}$

$\begin{array}{lcl}\sigma(\mu \mathrm{b} / \mathrm{sr}) & \text { Source } \\ 11.0 \pm 2.8 & \text { Ref. } 1 & \\ 12.8 \pm 4.4 & \text { Ref. } 5 & 12.1 \pm 2.7 \\ 13.45 \pm 3.4 & \text { Present work } & \\ 10.4 \pm 1.0 & \text { Ref. } 6 \\ 10.6 \pm 1.0 & \text { "best value" }\end{array}$

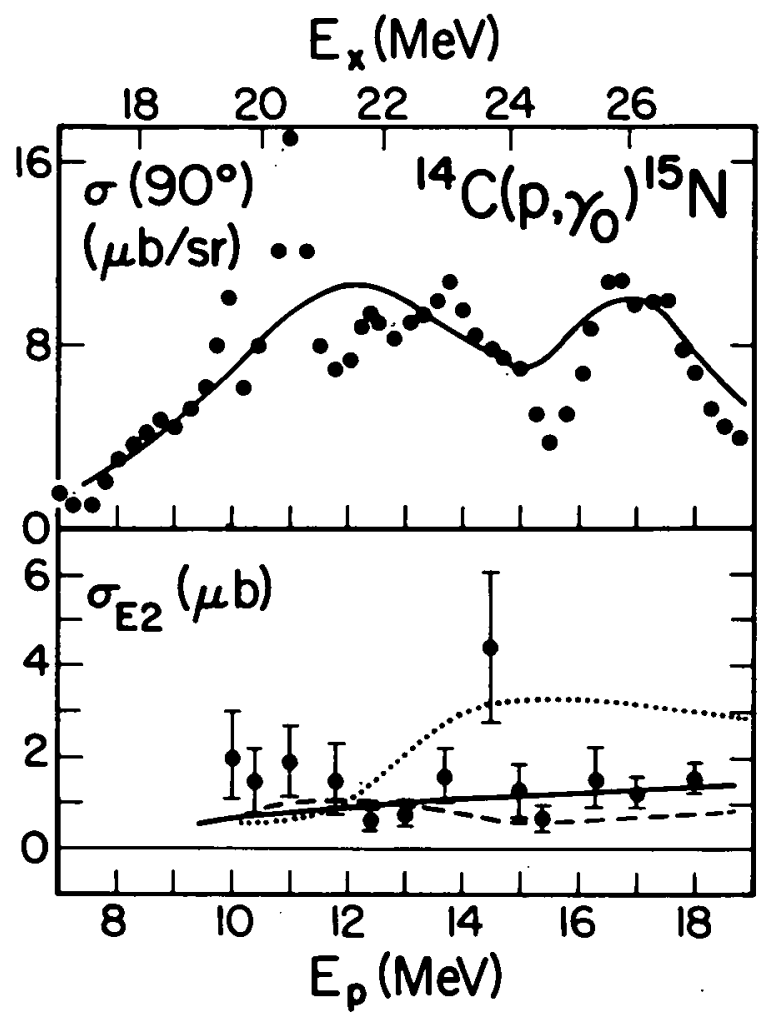

Fig. 9.2-4. The $90^{\circ}$ cross section (Ref. 1) and $\sigma_{E 2}$ derived from the present measurements.

The results are shown in Table 9.2-1. The errors in the present measurement $( \pm 25 \%)$ arc dominatied by the $120 \%$ error in the absolute ( $\mathrm{p}, \mathrm{p})$ cross section near $E_{p}=2.2 \mathrm{MeV}$ (Ref. 4). The top 3 entries are $(p, \gamma)$ determinations which suffer this same $\pm 20 \%$ uncertainty; the corresponding weighted average is indicated in the table. The fourth entry is derived from the ( $\left.e, e^{\prime} p_{0}\right)$ measurement of Ref. 6 . The "best" value is close to the number given in Ref. 1 , which is used in Fig. 4.

d. M.H. Harakeh et al., Pliys. Rev. C 12, 1410 (19\%5).

2. S.S. Hanna et al., Phys. Rev. Lett. 32, 114 (1974) and Proc. of Internat' $Z$ Conf. on Nuclear Structure, Amsterdam, 1974, Vol. 2, p. 249.

3. R.E. Marrs, E.G. Adelberger, K.A. Snover and M.D. Cooper, Phys. Rev. Lett. 35, 202 (1975).

4. W.R. Harris and J.C. Armstrong, Phys. Rev. 171, 1230 (1968).

5. H.R. Weller et al., - Phys. Rev. Lett. 32, 177 (1974); Phys. Rev.C 13,922 (1976).

6. J.J. Murphy, Y.M. Shin and D.M. Skopik, Nucl. Phys. A246, 221 (1975). 
K. Ebisawa and K.A. Snover

In the past year we have made additional development of the directsemidirect (DSD) reaction model and comparisons to experimental data on both proton and neutron capture in a variety of different cases in both light and heavy nuclei. The primary additional development has been investigations into the nature and suitability of various form factors, and the new applications in light nuclei are to the reactions $11_{B}(p, \gamma)^{12} \mathrm{C}$ and $14 \mathrm{C}(\overrightarrow{\mathrm{p}}, \gamma){ }^{15} \mathrm{~N}$.

\section{Studies of the Form Factor:}

Our explorations of the effects on the angular distribution of cross section and analyzing power of form factors of different radial shape shows that in all cases considered the major effect is in the magnitude of the resonance contribution. In light nuclei the phase difference between different channels is very insensitive to the choice of form factor, while the ratio of reaction amplitudes in different channels shows some dependence on the form factor. The different forms considered are $r f(r)$ and $d f(r)$ for $E l$, and $r^{2} f(r), r d f(r) / d r$ and $d f(r) / d r$ for E2, where $f(r)$ is the (volume) form of the optical potential.

Of particular interest, especially in light nuclei, is whether the use of these collective macroscopic form factors can be justified on a more microscopic basis. For E2 isoscalar excitations, the use of $\mathrm{dV}(r) / \mathrm{dr}$ or $\mathrm{rdV}(\mathrm{r}) / \mathrm{dr}$ has a reasonable theoretical basis. However, the use of hydrodynamic model form factors for isovector excitations in light nuclei is open to criticism, since, for example, the hydrodynamic model fails to give the correct excitation energy. Few detailed theoretical calculations of collective excilations give even the radial transition densities associated with these excitations. However, the response function calculations of Bertsch offer just such a basis for comparison. In the upper half of Fig. 9.3-1 we show the collective model "volume" and "surface" transition densities for the giant-dipole resonance of 160 , along with the response function calculation of Schlomo and Bertsch.l In the lower half of the figure are the form factors for the particle coupling matrix elements, where the response function result was obtained by folding the transition density with the zero-range density dependent interaction used 2 in the response function calculation. Thus both the shape and magnitude of the response function $F(r)$ and the volume collective model form factor are in close agreement.

Calculations of the ${ }^{11} B(p, y){ }^{12} C$ Reaction:

Here we use the Watson ${ }^{3}$ optical potentials, which were derived from a data set that included $I l_{B}(p, p) \perp \perp_{B}$ data. We define a radial matrix element for $E$ capture which is the sum of a direct and a semidirect (collective) part (the latter is treated in the single-level approximation):

$$
R_{\ell j}(\mathcal{L})=\left\langle r^{\mathcal{L}}{ }_{\ell j}+\alpha_{\mathcal{L T}}\left\langle F_{\mathcal{L T}}(r)\right\rangle_{\ell j} /\left[E-E_{\mathcal{L T}}+i \Gamma_{R T} / 2\right] .\right.
$$

The numerator in the second term represents the product of proton formation and $\gamma$-decay matrix elements, with 


$$
\left\langle F_{\ell T}(r)\right\rangle_{\ell j}=\int_{0}^{\infty} \frac{x_{\ell j}(r)}{r} F_{\ell T}(r) \frac{\phi_{\ell j^{\prime}}(r)}{r} r^{2} d r
$$

where $x_{\ell j}(r)$ is the initial proton scattering wave function and $\phi_{\ell}{ }^{\prime} j^{\prime}(r)$ is the wave function of the valence proton bound in the final state (the normalization of $\phi_{\ell} j^{\prime}(r)$ is given by the spectroscopic factor $\left.c^{2} S_{\ell} j^{\prime}\right)$. Here $R T$ labels the multipole and isospin of the collective excitation and $l j$ the quantum numbers of the projectile. For the "volume" Steinwedel-Jensen type form factor, $F_{11}(r)=$ $r V_{l}(r)$. where $V_{l}(r) / 4$ is the real symmetry term in the optical potential, with $V_{1}(0) \approx 100 \mathrm{MeV}$. For proton capture, $\alpha_{11}=3 \mathrm{~h}^{2} \mathrm{ZB}_{11} / 4 \mathrm{M}_{\mathrm{p}} \mathrm{A}\left\langle\mathrm{r}^{2}\right\rangle_{E_{11}}$ where $\beta_{11}$ is the fraction of the classical dipole sum rule exhausted by the resonance. A simple extension of the model permits one to include direct and collective E2 with a form factor for the latter given by $\mathrm{F}_{20}(r)=-\mathrm{rdV}_{0}(r) / \mathrm{d} r$ and $\alpha_{20}=$ $\mathrm{h}^{2} \mathrm{~B}_{20} / 2 \mathrm{M}_{\mathrm{p}} \mathrm{E}_{20}$ for an isoscalar resonance of strength $B_{20}$, where $V_{0}(r)$ is the real central potential $\left(F_{21}(r)=r^{2} V_{1}(r)\right.$ and $\alpha_{21}=5 h^{2}\left\langle r^{2}\right\rangle_{B_{21}} / 8 M_{p}\left\langle r^{4}\right\rangle E_{2 l}$ for an IV resonance).

The solid curves in Fig. 9.3-1 are obtained by including direct E2 and direct plus collective El. Here $E_{11}$, $\Gamma_{11}$, and $\alpha_{11}$ were adjusted to provide a fit (by eye) to the total cross section, with the result that $E_{11}=22.0 \mathrm{MeV}$, $\Gamma_{11}=4.0 \mathrm{MeV}$ and $\alpha_{11}=11.0$. The value of $\alpha_{11}$ calculated from the above relation with $V_{1}(0)=100 \mathrm{MeV}$ and $\beta_{11}=1.0$ is $\alpha_{11}=11.5$, in good agreement with the value determined by requiring a fit to. $\sigma_{\text {total. The calculated cross section }}$ is a bit high compared to the data for $7 \leq E_{p} \leq 12 \mathrm{MeV}$ and a bit low for $E_{p}>$ $12 \mathrm{MeV}$, indicating perhaps that the single-level approximation provides a good, but not perfect, fit. The measured angular distribution coefficients $a_{1}, a_{2}$ and $a_{3}$ are also well-described by the calculation. The calculated $\mathrm{b}_{2}$ coefficient drops from 0.04 to 0.00 over the range $8 \leq \mathrm{E}_{\mathrm{p}} \leq 13 \mathrm{MeV}$, whereas the measured value ${ }^{4}$ (not shown) is constant at about -0.1. The calculated $b_{2}$ involves interference contributions from $\mathrm{s}_{1 / 2} \mathrm{~d}_{3 / 2}, \mathrm{~s}_{1 / 2} \mathrm{~d}_{5 / 2}$ and $\mathrm{d}_{3 / 2} \mathrm{~d}_{5 / 2}$ which nearly cancel: the discrepancy may be explained if the calculation is somewhat underestimating the relative contribution of $s_{1 / 2}$, as was found in the cases of capture into $15^{\mathrm{N}}$ and 160 . The

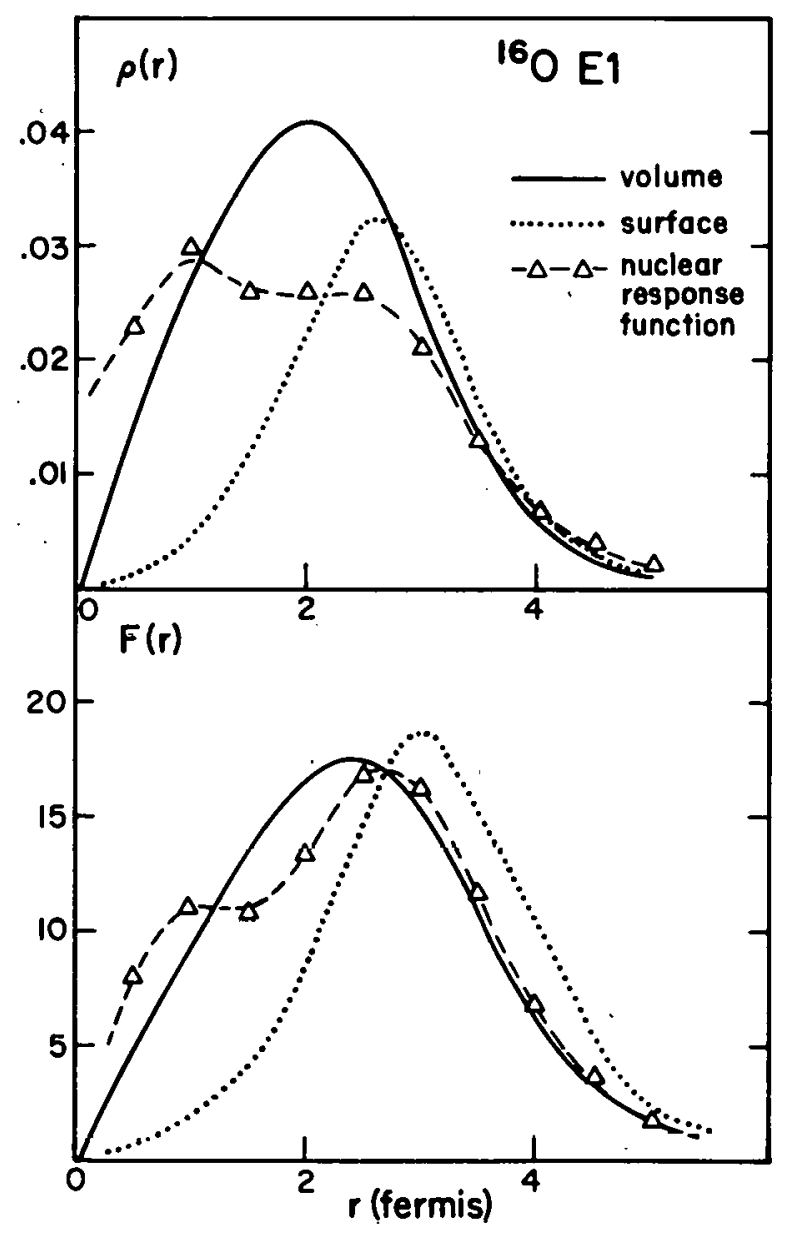

Fig. 9.3-1. Upper half: transition densities (normalized to the sum rule) for the $160 \mathrm{GDR}$. Lower half: the corresponding particle-coupling form factors. 
agreement with the measured $a_{1}$ and $a_{3}$ coefficients indicates most of the observed E1-E2 interference effects are due to direct E2 capture. The $a_{1}$ and $a_{3}$ are somewhat underestimated for $8 \leq \mathrm{E}_{\mathrm{p}} \leq 14 \mathrm{MeV}$, due in part to the overestimate of $\sigma_{\text {total. }}$. Also the calculation underestimates $\sigma_{\text {total }}$ at the highest energies by about $60 \%$, and thus might be expected to overestimate $a_{1}$ and $a_{3}$ by $230 \%$ if the discrepancy lies in the El cross section. Indeed, if we artificially.boost the magnitudes of the El amplitudes proportionally to get agreement with $\sigma_{\text {total }}$, then the calculated $a_{1}\left(a_{3}\right)$ at $E_{p}=29 \mathrm{MeV}$ becomes $0.68(-0.49)$ compared to the experimental values of $0.9(-0.5)$. Thus, although there may be some room for differences between the calculated and experimental $a_{1}$ and $a_{3}$ it is clear that most of the contributions arise from direct E2.

Thus there is no evidence from these data for significant amounts of collective E2 strength above the GDR. Even so, the direct capture is a significant contributor to the sum rules, with the cross section integral over $5 \leq E_{\mathrm{p}} \leq 30 \mathrm{MeV}$ yielding $20 \%$ of the isoscalar and $20 \%$ of the isovector sum rules. One can also use the model to estimate the sensitivity to a collective E2 resonance by making reasonable assumptions about the resonance energy, width and form factor', will the result that, for example, a collective isovector E2 resonance at $E_{X} \sim 35 \mathrm{MeV}$ with a width comparable to the GDR would show a strong effect in $(p, \gamma)$ if it contained most of the sum rule; if it contained less than half of the sum rule it would be hard to see. Thus most likley isovector E2 strength is either very much spread out, or it lies at energies beyond the reach of present experiments.

Calculations of the ${ }^{14} \mathrm{C}(p, \gamma)^{15} \mathrm{~N}$ Reaction:

Here we have performed calculations similar to those described for the $11_{B}(p, \gamma)^{12} \mathrm{C}$ reaction. For the ${ }^{14} \mathrm{C}(\overrightarrow{\mathrm{p}}, \gamma)^{15} \mathrm{~N}$ reaction we used optical potentials derived from Ref. 5 using the $N$ and $Z$ dependence given in Ref. 3 .

The fragmentation of El strength as seen in Fig. 9.2-4 precludes a single level fit which will describe both the GUK region and the high-energy tall aluove $E_{p}=18 \mathrm{MeV}$. However the introduction of a second single-level El amplitude permits the fit shown in Fig. 9.2-4 (solid line) with $E_{11}=21.0(25.5) \mathrm{MeV}, \Gamma=$ $6.0(2.0) \mathrm{MeV}$ and $\mathrm{V}_{l}(0) B_{11}=115(25) \mathrm{MeV}$ for the two resonances. The solid curves in the figures of Sec. 9.2 also contain direct E2 and provide a good description of the various $a_{i}$ and $b_{i}$ coefficients, thus providing support for the neglect of $M 1$ radiation in the model-independent extraction of $\sigma_{E 2}$ from the data (we have also calculated Ml direct capture and found it to be negligible).

Various authors have speculated that this upper fragment observed in the $90^{\circ}$ cross section near $E_{\mathrm{p}}=17 \mathrm{MeV}$ is primarily $\mathrm{T}_{>}$. The data of Fig. 9.2-2 show little evidence for a change in structure in this energy region, since, except for some fluctuations the relative $s$ - and d-wave amplitudes and relative phases are quite constant as a function of energy. The most significant deviation from the calculation is, however, in the variation of the phase above $E_{p}=15 \mathrm{MeV}$ (assuming the solution indicated by the dots). This is surprising since our experience with different form factors indicates the relative phase is very hard to change. In any case, the strength in this fragment relative to that at lower energies is much lower than the ratio of $1: 1$ predicted for pure $T_{>}: T_{<}$splitting. 
In order to gauge the sensitivity of $(\vec{p}, \gamma)$ to possible collective E2 strength, we also show in Figs. 9.2-1 and 9.2-4 model predictions for an IS resonance at $E_{0}=22 \mathrm{MeV}, \Gamma=4 \mathrm{MeV}$ exhausting $50 \%$ of the IS sum rule $\left(B_{20}=0.5\right)$, and a prediction for a similar IV resonance exhausting $100 \%$ of the IV sum rule $\left(B_{21}=1.0\right)$. No theoretical calculation ${ }^{4}$ places much IV strength at these low energies and indeed a concentrated IV resonance such as is shown in Fig. 9.2-4 would be in clear disagreement with the data. However, the DSD model predicts that a rather weak resonance effect in $(\vec{p}, \gamma)$ would result from a concentration of IS E2 strength. Thus the situation in $14 \mathrm{C}(\overrightarrow{\mathrm{p}}, \gamma)^{15} \mathrm{~N}$ seems to offer no surprise, and indeed is reasonably well understood in terms of the calculations. The big puzzle is why the situation appears so drastically different in 160 .

1. S. Schlomo and G. Bertsch, private communication.

2. G. Bertsch and S.F. Tsai, Phys. Reports 18C, 126 (1975).

3. B.A. Watson et al., Phys. Rev. 182, 977 (1969).

4. H.F. Glavish et al., Phys. Rev. Lett. 28, 766 (1972).

5. B.M. Skwiersky et al., Phys. Rev. C 9, 910 (1974).

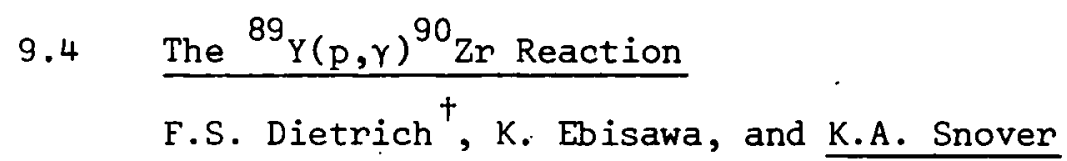

Continued experimental and theoretical investigation of the ${ }^{89} \mathrm{Y}(\mathrm{p}, \mathrm{\gamma})^{90} \mathrm{Zr}$ reaction is being carried out in a collaboration with Livermore. In addition to data taken in this Laboratory, $l$ seven-point angular distributions were measured at Livermore in $2 \mathrm{MeV}$ steps from $E_{\mathrm{P}}=17$ to $25 \mathrm{MeV}$. These data show large $\mathrm{a}_{3}$ $\approx-0.6)$ and $\left.a_{4} \approx-0.4\right)$ coefficients at the higher energies, along with some evidence for a non-zero $a_{5}$ coefficient at $E_{p}=23 \mathrm{MeV}$.

DSD model calculations including direct E2 and E3 along with direct plus collective El provide a good fit to the total cross section and angular distribution coefficients above $E_{\mathrm{p}}=10 \mathrm{MeV}$. A strong isovector $\mathrm{GQR}$ ( $100 \%$ of the sum rule and $\Gamma \sim 4 \mathrm{MeV}$ ) near the expected excitation energy of $\sim 27 \mathrm{MeV}$ is incompatible with the data, while a much weaker resonance as has been suggested by electron scattering would not disagree with the data. The inclusion of direct E3 markedly improves the agreement between the calculated and experimental $\alpha_{2}$ values $\left\{\alpha_{2}=2\left[1-2 Y\left(90^{\circ}\right)\right] /\left[Y\left(55^{\circ}\right)+Y\left(125^{\circ}\right)\right]\right\}$, and predicts a small negative $a_{5}$ at the highest energies, as suggested by experiment.

The data require a collective $E l$ volume strength factor $V_{1}(0) B_{11} \approx 200 \mathrm{MeV}$ assuming a purely real transition potential. The origin of such a large coupling strength is not clear. With this strength, the total cross section is fit well above $E_{p}=10 \mathrm{MeV}$. At lower energies compound processes are important and the inclusion of a Hauser-Feshbach compound contribution provides a good fit to measured $a_{2}$ and $b_{2}$ coefficients down to $E_{p}=6 \mathrm{MeV}$.

A calculation of capture to the excited-state group " $\gamma_{1-5}$ ", expected to be dominated by the $g_{9 / 2}$ final state, yields a resonance shape which is distorted in much the same way as was found in $208 \mathrm{~Pb}(\mathrm{p}, \gamma)$ and $208 \mathrm{~Pb}(\mathrm{n}, \gamma)$; namely the 
calculation is too low on the low energy side of the GDR and too high on the high energy side. Thus the shape problems in the DSD calculations seem to be associated with capture to high-spin final states .

$+\quad$ Permanent address: Lawrence Livermore Laboratory, Livermore, CA.

1. Nuclear Physics Laboratory Annual Report, University of Washington (1974), p. 142 .

9.5 Direct-Semidirect Calculation in the $\mathrm{Pb}$ Region

K. Ebisawa and K.A. Snover

We have applied Direct-Semidirect (DSD) radiative capture theory to the heavy nuclei $205 \mathrm{Tl}$, and $208 \mathrm{~Pb}$, where the isovector Giant Quadrupole Resonance $(G Q R)$ is expected at a relatively low excitation energy of about $120 \sim 130 \mathrm{~A}^{-1 / 3}$ $\mathrm{MeV}$ or $\sim 23 \mathrm{MeV} .1,2$ Experimental results for the reaction $(p, \gamma)$ on these nuclei show a considerable amount of interference of $E 1$ with $E 2^{3-5}$ above the Giant Dipole Resonance (GDR), but it is not evident that this quadrupole strength comes from a GQR. It is, thus, necessary to estimate the total dipole strength and the direct quadrupole contribution in order to investigate the GQR. We used the DSD mode $1^{6}$ and only results and a few remarks are presented here.

Optical potentials to generate the continuous state wave functions were taken from the analysis by Becchetti and Greenlees. 7 The bound state wave functions were obtained from the same optical parameters by changing the real potential depth (with no imaginary part). Direct reaction amplitudes are immediately obtained from these wave functions and the electric multipole operator $f_{\rho}(r)$. The long wave approximation $f(r)=r^{\mathscr{L}}$ was found to be a good one up to about $30 \mathrm{MeV}$ excitation energy. Both volume and surface forms of the collective form factor were used, and it was found that the choise of form factor affected only the magnitude, but not the shape of the resonance cross section. In the following results we deal exclusively with the volume form. The dipole resonance energy and its width were chosen empirically as $E_{R}=13.5 \mathrm{MeV}, \Gamma_{\mathrm{R}}=4.0 \mathrm{MeV}$. For demonstration purposes, an isovector quadrupole resonance was chosen at $E_{R}=24 \mathrm{MeV}$ with $\Gamma_{R}=4 \mathrm{MeV}$ in our present calculations.

The following two quantities are shown in figures;

(1) The total cross section

$\sigma_{t} / 2 \pi \approx \sigma\left(55^{\circ}\right)+\sigma\left(125^{\circ}\right)$

and the asymmetry.

$$
\begin{aligned}
a & \equiv 2 \pi\left[\sigma\left(55^{\circ}\right)-\sigma\left(125^{\circ}\right)\right] /\left[\sigma_{t} P_{1}\left(55^{\circ}\right)\right] \\
& \approx a_{1}-0.68 a_{3}
\end{aligned}
$$

where the $\mathrm{A}_{4}$ coefficient in the Legendre expansion has been neglected.

Figure 9.5-1 shows the above quantities for the ${ }^{205} \mathrm{~T} \ell\left(\mathrm{p}, \gamma_{0}\right){ }^{206} \mathrm{~Pb}$ reaction. The experimental $\gamma$-ray yield was obtained by making a least squares line shape Iit to the $\gamma$-ray spectrum. 


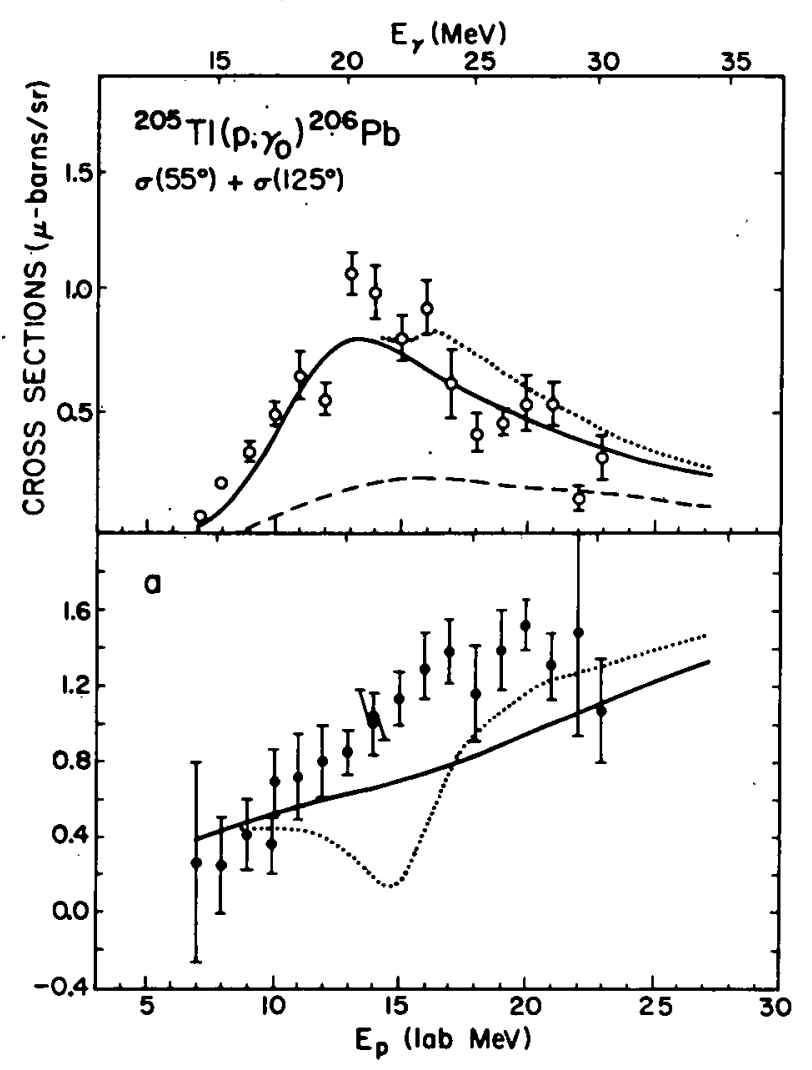

Fig. 9.5-1. The points represent the measured cross section $\sigma\left(55^{\circ}\right)+\left(125^{\circ}\right)$ $\sigma($ total $/ 2 \pi$ and asymmetry "a" for the ${ }^{205} \mathrm{~T} \ell\left(\mathrm{p}, \mathrm{Y}_{\mathrm{O}}\right){ }^{206} \mathrm{~Pb}$ reaction. The dashed line represents the calculated direct El plus direct E2 cross section. The solid line is a DSD calculation including direct E2 and direct plus collective El. The dotted line includes a collective isovector E2 resonance (see text).

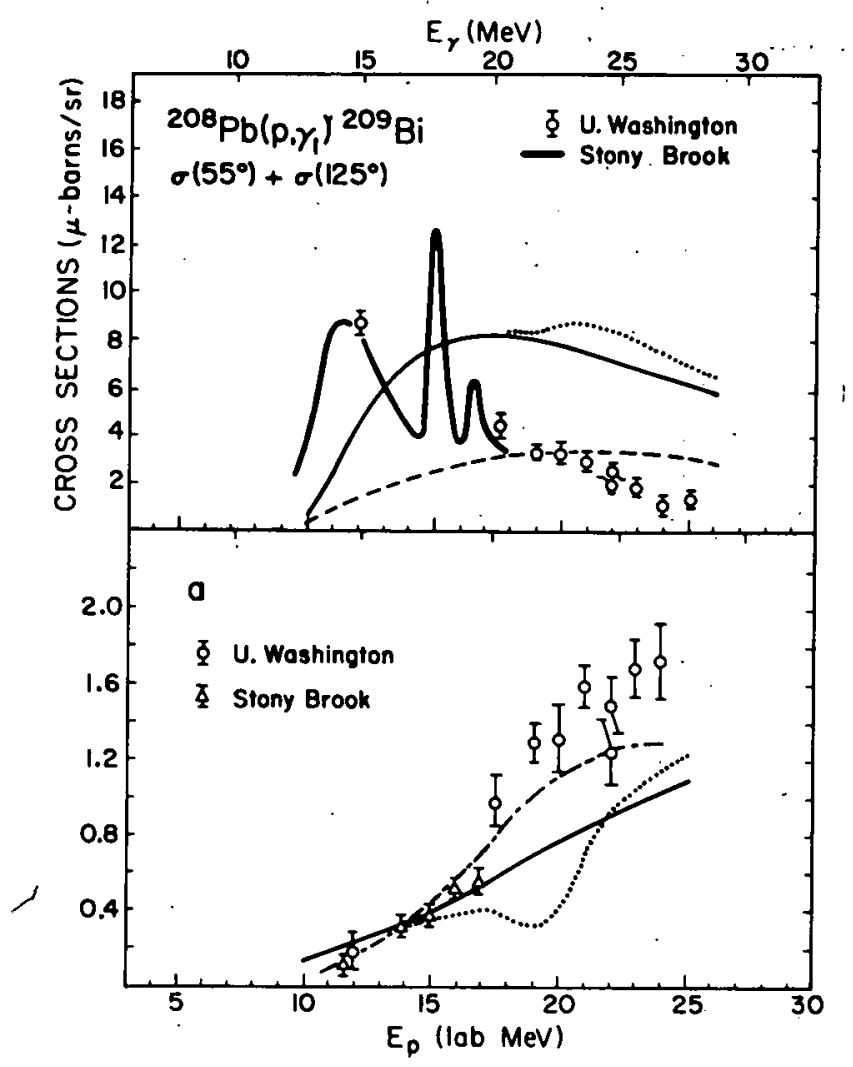

Fig. 9.5-2. Here we display the same quantities as in Fig. 9.5-1 for the $208 \mathrm{~Pb}\left(\mathrm{p}, \gamma_{1}\right)^{209} \mathrm{Bi}$ reaction. The broad line and triangles represent data taken from Ref. 5. The dot-dash line in the lower part of the figure results from a DSD calculation including direct $E 2$ and direct plus collective El, with the El amplitudes adjusted to fit the total cross section.

The excitation function of the cross section is well described by total El with direct E2 (solid line). Here this total El cross section consists of direct El and a GDR with strength of $V_{1}(0) B_{11}=150 \mathrm{MeV}$. A GQR resonance with $E_{R}=$ $24 \mathrm{MeV}, \Gamma_{R}=4 \mathrm{MeV}$ and $V_{l}(0) \beta_{2 l}=100 \mathrm{MeV}$ is included in the calculation shown as a dotted line. However the observer cross sections contain contributions from other processes such as capture through Isobaric Analog Resonances, so that it is difficult to evaluate the strength of a GQR from the total cross section data only. If neither GDR nor GQR were present, the direct reaction would produce a very small cross section (dashed line) with a broad peak around $E_{p} \sim 16 . \mathrm{MeV}$. Direct E2 itself has a broad peak around $E_{p} \sim 25 \mathrm{MeV}$ and contributes about $50 \%$ of total direct cross section there.

The same kinds of calculations for ${ }^{208} \mathrm{~Pb}\left(\mathrm{p}, \gamma_{1}\right){ }^{209} \mathrm{Bi}$ are shown in Fig. 9.5-2 
with the same notation. Here total El with or without collective E2 does not properly describe the gross structure of the data. Even the direct cross sections themselves exceed the experimental values from $E_{p} \sim 19$ to $24 \mathrm{MeV}$.

It should also be mentioned that for both the direct magnetic dipole (MI) reaction and the direct electric octupole (E3) one has more than one order of magnitude smaller cross sections than for the direct E2 in these nuclei, and can be reasonably neglected in our analysis.

The front-to-back asymmetry "a" is a sensitive way to look for a quadrupole resonance. The interference of direct E2 with total El yields a monotonically increasing asymmetry as shown by solid lines in both figures. Those lines quite agree with data up to few $\mathrm{MeV}$ above GDR. Note that if total El amplitudes are re-normalized channel independently to fit the experimental total cross section of $208 \mathrm{~Pb}\left(\mathrm{p}, \gamma_{1}\right)^{209} \mathrm{Bi}$, the calculated asymmetry becomes very similar to the data (dashed and dotted line in Fig. 9.5-2). The departure of data from this line might be due to a resonance-like $G Q R$, but the calculated effect of such a resonance results in destructive interference just below the GQR (dashed line). The introduction of direct $\mathrm{MI}$ and direct E3 changes the asymmetry by an amount of $10 \%$. Whatever isovector $G Q R$ parameters are assumed, the symmetric shape seen in the asymmetry measurement could be obtained only if the relative phase between the total El and the collective $\mathrm{E} 2$ is different than that given by the model.

Finally we present a radiative neutron capture calculation together with the data taken from I. Bergquist et al.8 Figure 9.5-3 shows the total cross sections. The absence of the Coulomb potential results in a very smooth direct capture cross section (dashed line), to which the direct E2 contributes a negligible amount, due in part to the negligible size of the effective charge of a neutron for $\mathrm{E} 2$, i.e., $\mathrm{Z} / \mathrm{A}^{2}$.

The collective El strength needed to produce the solid curve shown in Fig. 9.5-3 is $V_{1}(0) B_{11}=250 \mathrm{MeV}$. However, the shape is given incorrectly (see below). Here we use the resonance energy $E_{R}=13.5 \mathrm{MeV}$ and width $\Gamma_{R}=4.0$ $\mathrm{MeV}$ as determined from photonuclear measurements. One could get a better fit to the low energy region by shifting the resonance energy, but we don't consider. such a parameter adjustment as permissible. In addition we must also investigate whether the compound process can contribute significantly to the low energy region.

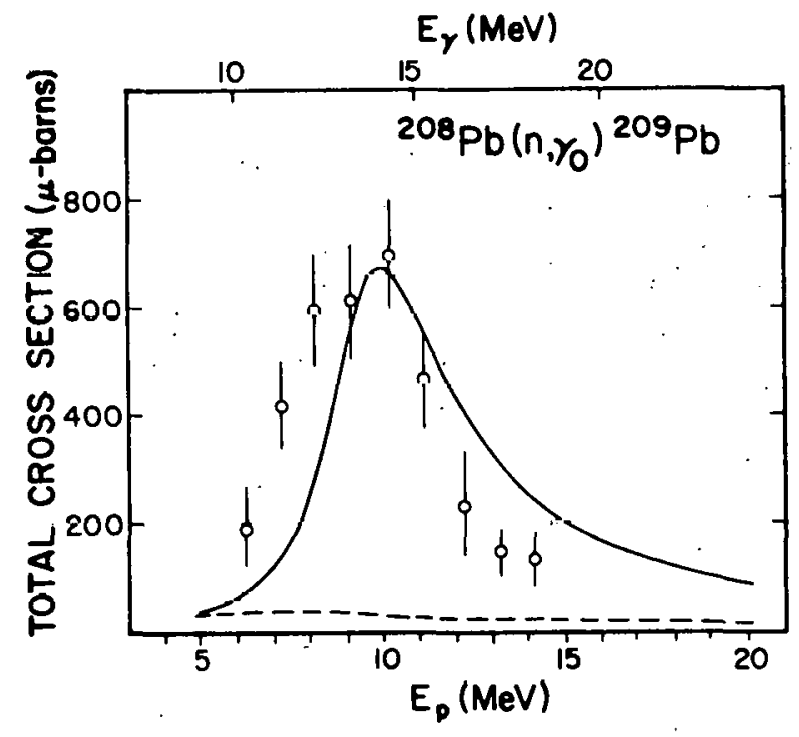

Fig. 9.5-3. DSD calculation (solid line) and measured data (points taken from Ref. 8) for the total cross section for the $208 \mathrm{~Pb}\left(\mathrm{n}, \mathrm{\gamma}_{0}\right)^{209} \mathrm{~Pb}$ reaction. The dashed line represents the calculated direct $E l$ plus direct E2 cross section. 
It is interesting to note that the asymmetry due to total El and direct E2 is strongly suppressed by the effective charge, so that the $\gamma$-ray angular distribution would be expected to be symmetric around $90^{\circ}$. Any asymmetry must come from other processes such as GQR. Only a few experimental results are available for lighter nuclei. 9

The problems where the DSD model fails to give a reasonable description of the total cross section seem to be associated with capture into final singleparticle orbitals with large $\ell, j$. The discrepancies get progressively worse for the cases of capture into the $\mathrm{lh}_{\mathrm{g} / 2}$ and $1 i_{13 / 2}$ proton orbitals in $209_{\mathrm{Bi}}$. In addition a similar effect is found for neutron capture. The fact that a much bigger collective strength is needed in the case of $208 \mathrm{~Pb}(n, \gamma){ }^{209} \mathrm{~Pb}$ as compared to $205 \mathrm{~T} \ell(p, \gamma)^{206} \mathrm{~Pb}$ may also be associated with this problem. We are presently trying to understand the cause of these difficulties.

1. $\quad$ T. Suzuki, Nucl. Phys. A217, 182 (1973).

2. A. Bohr and B. Mottelson, Nuclear Structure, Vol. 2 (Benjamine, 1975).

3. K.A. Snover et al., Phys. Rev. Lett. 32, 317 (1974).

4. Nuclear Physics Laboratory Annual Report, University of Washington (1975), p. 170 .

5. J.F. Amann, Ph.D. Thesis, State University of New York at Stony Brook, 1972, unbpublished.

6. See Sec. 9.3 of this report.

7. F.D. Becchetti, Jr., and G.W. Greenlees, Phys. Rev. 182, 1190 (1969).

8. I. Bergqvist et al., Nucl. Phys. A191, 641 (1972).

9. E.D. Arthur et al., Phys. Rev. Lett. 35, 914 (1975).

9.6 Radiative Capture of Fast Neutrons

E. Arthur ${ }^{\dagger}$, D. Drake ${ }^{\dagger}$, and I. Halpern

In earlier reports ${ }^{1}$ we have stressed the special feature of neutrons as projectiles for studies of radiative capture -- namely that, in distinction to charged particles, they have negligible amplitudes for direct capture in electric quadrupole and higher modes. As a result they are uniquely suited to:display any collective quadrupole and higher amplitudes that may be present, since there are no direct amplitudes to obscure them.

We have already reported our investigation of neutron capture in some lighter elements using $14 \mathrm{MeV}$ neutrons. 2 These results are not very easy to understand and we decided to extend the measurements by (1) dropping to lower energies for the incident neutrons and (2) studying heavier targets. The reason for going to heavier targets is that there are evidences for more or less classical behavior (i.e., localization in excitation energy) of the higher multipole giant resonances in such nuclei whereas the lighter nuclei seem, for some reason, to have their higher resonances all spread out in energy. Unfortunately it is hard to resolve capture lines in heavy targets because even the low lying levels are so close together. The level separations in lead are however ample and it was decided to study neutron capture in $\mathrm{Pb}$. 
The point of using a lower (and variable) energy neutron source was to be able to span the region where the expected isoscalar E2 resonance lies.

Lower energy measurements on $\mathrm{Pb}$ present a number of technical difficulties that were not present in our runs using $14 \mathrm{MeV}$ neutrons on light targets. 2 (1) The neutron fluxes are generally not as large at low energies as with the popular $\mathrm{d}+\mathrm{t} 14 \mathrm{MeV}$ neutron source. (2) In the low energy measurements one must work in the charged-particle beam plane with the consequent trouble that back-. grounds due to the neutron production target depend strongly on the angle of observation. This makes background subtraction in angular distribution studies difficult. (3) Targets as heavy as lead which are large enough to allow the measurement of a capture cross-section are also large enough to attenuate the capture photons considerably. This also interferes with angular distribution studies.

Last summer we used the LASL vertical electrostatic generator to see whether it was possible to overcome some of these difficulties. We learned in the course of our investigation that it was necessary (and possible) to monitor continuously and with precision the pressure in the neutron production gas target. We also learned that it was possible to shield adequately against production target backgrounds and to correct. for photon attenuation in the capture target. These encouraging findings were obtained in carbon and lead runs in which we measured the fore-aft ratios for $n, n^{\prime} \gamma$ photons from the $4.4 \mathrm{MeV}$ level in $12 \mathrm{C}$ and the 2.6 level in $\mathrm{Pb}$. After we corrected for Doppler shifts and attenuation in the target, the yields of these photons at supplementary angles fore and aft were found to be equal, as they are expected to be. In the very same measurements we also observed capture radiation. Our preliminary results for $7 \mathrm{MeV}$ neutrons show the radiations in carbon to be virtually symmetric fore and aft (no evidence for E2 capture) but asymmetric with the lead target. We are looking forward to a chance to pursue our work with lead, to gather more statistics, and to study the capture distribution as a function of incident neutron energy.

$+\quad$ Permanent address: Los Alamos National Laboratory, Los Alamos, NM 87544.

1. Nuclear Physics Laboratory Annual Report, University of Washington (1975), P. 175 .

2. E.D. Arthur, D.M. Drake, and I. Halpern, Phys. Rev. Lett. 35, 914 (1975). 
10. SCATTERING AND REACTIONS

-10.1 Excitation Functions and Angular Distributions for Inelastic ${ }^{4}$ He Scattering to the $\left(0^{+}, 6.44 \mathrm{MeV}\right) \frac{\text { Y State in }{ }^{24} \mathrm{Mg}}{!}$

K.G. Bernhardt, J.S. Blair, H. Bohn, J.G. Cramer, B. Cuengco, and

R. Sielemann

Last summer Morsch, Dehnhard, and $\mathrm{Li}^{1}$ reported measurements of the forward angle differential cross sections for the reactions ${ }^{24} \mathrm{Mg}\left(\alpha, \alpha^{\prime}\right)^{24} \mathrm{Mg}\left(0^{+}, 6.44 \mathrm{MeV}\right)$ and ${ }^{28} \mathrm{Si}\left(\alpha, \alpha^{\prime}\right) 28 \mathrm{Si}\left(0^{+}, 4.95 \mathrm{MeV}\right)$. at a bombarding energy of $23.5 \mathrm{MeV}$ and went on to analyze these results in terms of a microscopic DWBA model. They found that their theoretical angulan distributions were sensitive to the nature of the particlehole components in the ground and excited state wave functions and argued that reasonable fits to their data required that there be sizable lp hole components. If one grants the validity of their analysis, one is then furnished with a sensitive tool for examining the character of monopole excitations.

Some previous experiments, conducted here and elsewhere, have made us skeptical, however, that an interpretation of the observed inelastic $\alpha$ scattering in terms of a purely direct reaction mechanism is valid for such targets, states, and incident energy. For example, the inelastic angular distributions for excitation of the lowest $2^{-}$and $4^{+}$levels in 160 varied non-uniformly as the incident energy was stopped between 35.4 and $41.0 \mathrm{MeV} .{ }^{2}$ Back angle excitation functions for elastic and inelastic scattering on $24 \mathrm{Mg}$ of $\alpha$-particles with incident energy in the range 17.7-27.0 MeV display strong fluctuations with a coherence width approximately equalling $100 \mathrm{keV} .3$ The angular distributions for excitation of the $3^{+}$level at $5.22 \mathrm{MeV}$ show marked fluctuations even at forward angles and the energy average over the range 19 to $22 \mathrm{MeV}$ is consistent with the predictions of a statistical Hauser-Feshbach calculation. 4

In an effort to determine how large is the compound nuclear contribution to the weak monopole excitation in ${ }^{24} \mathrm{Mg}$, we have measured excitation functions over the range 22.3 to $24.2 \mathrm{MeV}$ as well as angular distributions at selected energies. Unfortunately, there was considerable $C$ and 0 contamination in the target so that our useful data were restricted to the angular range, $35^{\circ} \leq \theta_{1 a b} \leq$ $80^{\circ}$.

In Fig. 10.1-1 we show the excitation function at $\theta_{\text {lab }}=35^{\circ}$ 'for inelastic scattering into the monopole state. This angle corresponds to the third maximum in the angular distribution at $E_{\alpha}=23.5 \mathrm{MeV} .1$ It is seen that within the $1 \mathrm{MeV}$ interval above $23 \mathrm{MeV}$ the cross sections vary by more than a factor of 2 while between 22.6 and $22.8 \mathrm{MeV}$ the cross section increases by a factor of 5 .

Angular distributions for elastic scattering and inelastic excitation of the $6.44 \mathrm{MeV}$ level at 5 incident energies between 22.3 and $23.5 \mathrm{MeV}$ are shown in Fig. 10.1-2. The open circles in the inelastic angular distribution at $23.5 \mathrm{MeV}$ are the results of Ref. 1 and the corresponding dashed curve is their DWBA calculation. The dashed curve to the left is the calculated elastic angular distribution for elastic scattering at $23.5 \mathrm{MeV}$ using the optical model parameters 

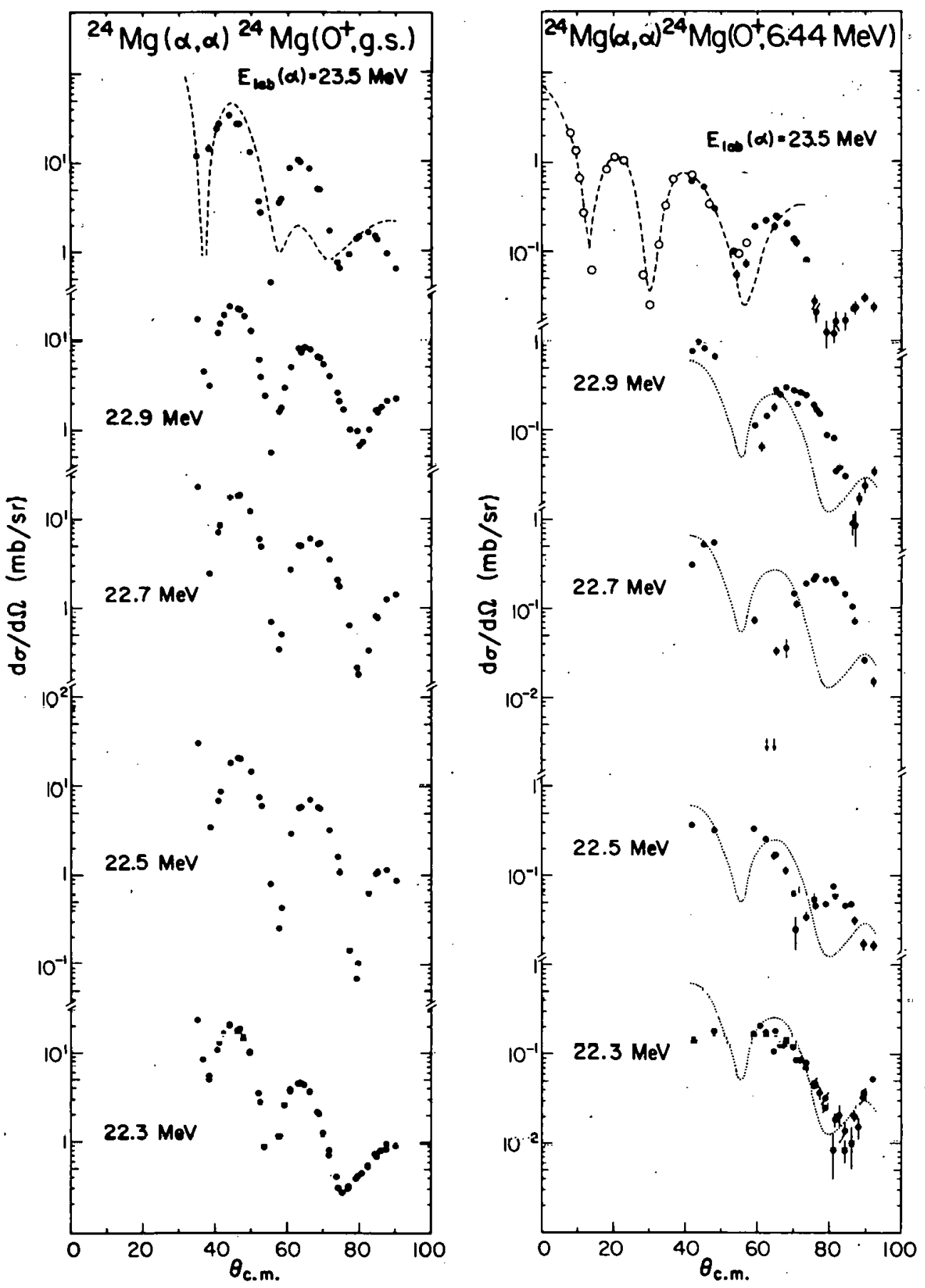

Fig. 10.1-2. Angular distributions for elastic scattering and inelastic scattering to the $0^{+}$level of $24 \mathrm{Mg}$ at $6.44 \mathrm{MeV}$ for five different incident energies. The graphics are explained in the text. 
listed in Ref. 1. We note that our extended data for the inelastic scattering no longer agree with the DWBA calculations at the larger angles. Further, the optical model fit to the elastic scattering data is worse than mediocre and makes suspect the DWBA calculations based on these parameters.

Not surprisingly, the elastic angular distributions are fairly stable at these energies. There are marked differences, however, in the inelastic patterns. We note, for example, that the phase of the oscillatory pattern shifts so that the maximum which occurs at $65^{\circ}$ when $E_{\alpha}=23.5 \mathrm{MeV}$ has become a minima at the same angle when $E_{\alpha}=22.7$ $\mathrm{MeV}$. To facilitate making a comparison, dotted curves which represent the cross sections measured at $23.5 \mathrm{MeV}$ are shown along with the data at the four lower energies.

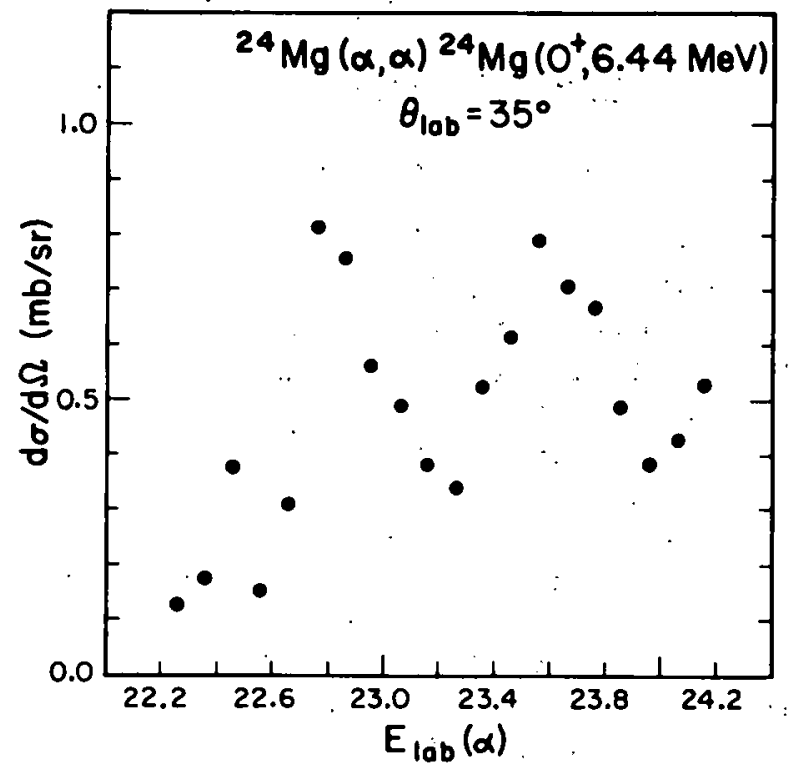

Fig. 10.1-1. Excitation function for ${ }^{24} \mathrm{Mg}\left(\alpha, \alpha^{\prime}\right)^{24} \mathrm{Mg}\left(0^{+}, 6.44 \mathrm{MeV}\right) \cdot$ at $\theta_{\mathrm{lab}}=$ $35^{\circ}$.

Our data in hand do not include the regions around $15^{\circ}$ and $30^{\circ} \mathrm{c.m}$. where Morsch et al. argue that fits to their observed deep minima require a sizable $1 \mathrm{P}_{1} / 2$ hole component. It is yet conceivable that the inelastic angular distributions forward of $35^{\circ}$. will show only the slow variation with energy expected for purely direct reactions but the present incomplete results strongly suggest that this is not the case.

1. H.P. Morsch, D. Dehnhard, and T.K. Li, Phys. Rev. Lett. 34, 1527 (1975).

2. Nuclear Physics Laboratory Annual Report, University of Washington (1965), P. 3 .

3. J.D.A. Roeders, Thesis, Groningen (1971).

1. A.C. Drentje and J.D.A. Roeders, Fliys. Lell. 38B, 356 (1970). 


\section{REACTIONS WITH POLARIZED PROTONS AND DEUTERONS}

\subsection{A Tensor Deuteron Beam Polarimeter Based on the ${ }^{3} \mathrm{He}(\mathrm{d}, \mathrm{p})^{4} \mathrm{He}$ Reaction}

N.L. Back, L.D. Knutson, W. Lynch, and T.A. Trainor

3 We report construction of a deuteron beam tensor polarimeter based on the $3 \mathrm{He}(\mathrm{d}, \mathrm{p}){ }^{4} \mathrm{He}$ reaction. This polarimeter measures the beam tensor polarization and spin-axis orientation angle by means of an array of five detectors. A preliminary calibration has been carried out between 11.5 and $18 \mathrm{MeV}$. This work complements a previous study of the ${ }^{4} \mathrm{He}(d, d){ }^{4} \mathrm{He}$ reaction as a deuteron vector polarization analyzer up to $18 \mathrm{MeV} .1$

The tensor polarimeter is shown in Fig. 11.1-1. It consists of a coliimator and electron suppression system, a 3 He gas cell, slowing foils and an array of five Si(Li) detectors. The primary deuteron beam, after passing through the target of interest, is incident on a 1/16"-diameter suppressed collimator where most of the beam stops and can be integrated. A small amount of the beam passes through the aperture and into the ${ }^{3} \mathrm{He}$ cell. This cell operates at $35-40$ PSIA and has a $1 / 4 \mathrm{mil}$ havar entrance foil mounted on a 1/4" radius. The exit foil is composed of a $15 \mathrm{mil}$ tantalum foil spot-welded to a 5 mil stainless-steel foil which forms the vacuum seal. These are mounted on a 1/2" radius. The exit foil is thick enough to stop deuterons at all incident beam energies, and provision is made to integrate total charge incident on the cell.

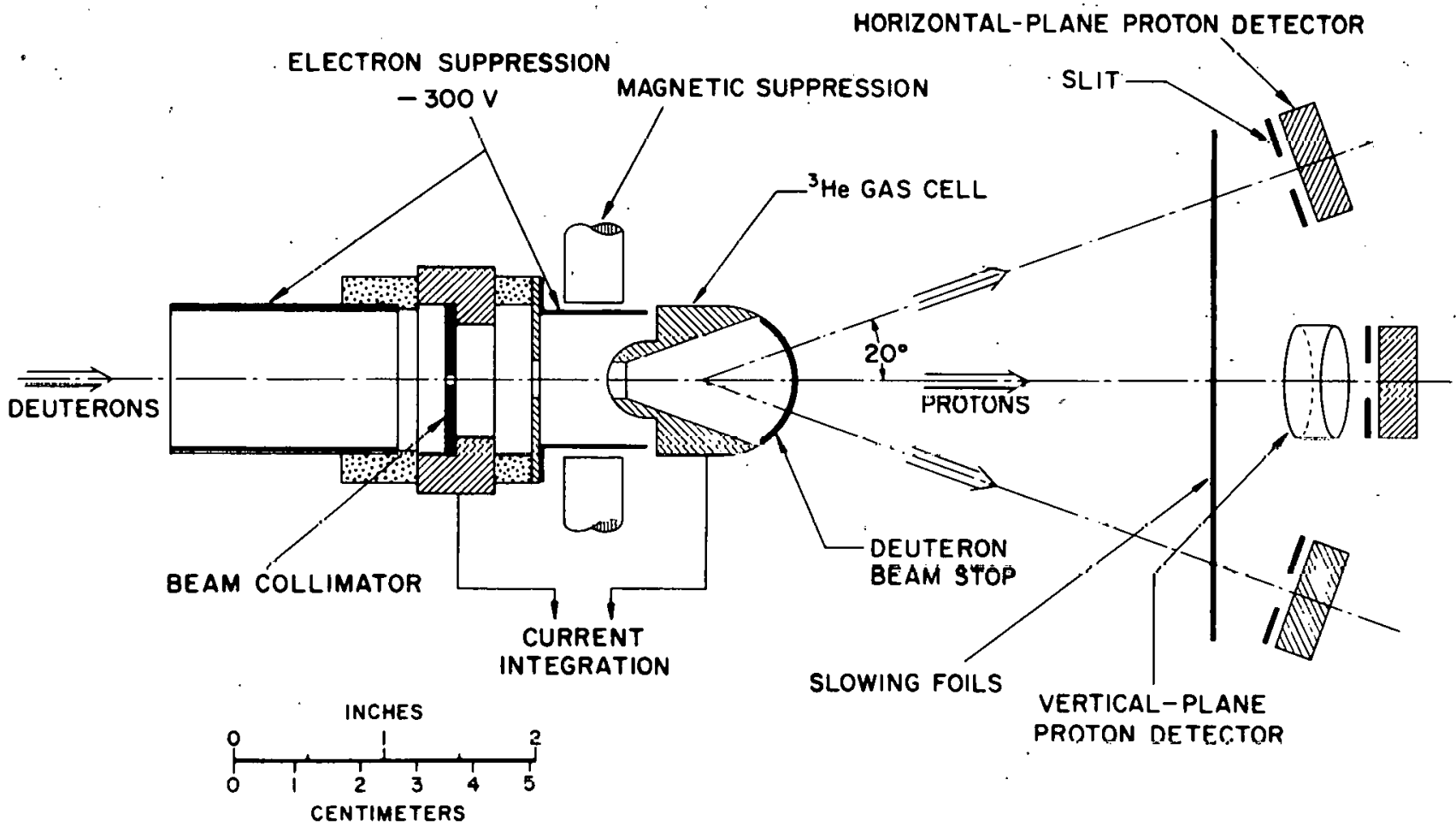

Fig. 11.1-1. Deuteron Tensor Polarimeter. 
Protons from the ${ }^{3} \mathrm{He}(\mathrm{d}, \mathrm{p})$ reaction have a positive $Q$ value of more than $18 \mathrm{MeV}$ and so easily pass through the gas cell exit foil. Slowing foils are placed in front of the detector array to insure that the protons lose all or almost all of their energy in the detector active areas. When $4 \mathrm{~mm}$ thick $\mathrm{Si}(\mathrm{Li})$ detectors are used in the array a single $15 \mathrm{mil}$ stainless-steel slowing foil is required for incident beam energies of $12-18 \mathrm{MeV}$ and no foil is required below $12 \mathrm{MeV}$.

The detector array consists of 5 detectors with 1/4"-diameter collimators placed 4.5" from the center of the gas cell. One detector is at $0^{\circ}$ and four detectors are at $20^{\circ}$, placed symmetrically in horizontal and vertical planes as shown in Fig. 11.1-1. The 200 laboratory angle was determined from preliminary measurements of tensor analyzing powers for ${ }^{3} \mathrm{He}(\mathrm{d}, \mathrm{p})$ which showed that $\dot{\mathrm{T}}_{21}$, the most important analyzing power for determination of the spin angle, has a maximum value near $20^{\circ}$ over a broad range of energies up to $18 \mathrm{MeV}$.

Proton yields to the four $20^{\circ}$ detectors with polarized beam incident are denoted $L, R, U, D$. With unpolarized beam a subscript zero is added. Similarly $A$ (for along) and $A_{0}$ denote yields to the $0^{\circ}$ detector. Quantities $E_{L}, E_{R}, E_{U}$, $E_{D}, E_{A}$ which are independent of detector efficiencies are formed as, for instance, $E_{\mathrm{L}}=\mathrm{L} / \mathrm{L}_{0}$. These five ratios can be combined to yield tensors representing the beam polarization in the target coordinate system. It is assumed that the spin axis lies in the horizontal plane but that the angle between the spin axis and beam axis, $\beta$, is arbitrary. The spin tensors are given by

$$
\begin{gathered}
t_{20}=\frac{E_{A}-1}{T_{20}\left(0^{\circ}\right)}=\frac{E_{L}+E_{R}+E_{U}+E_{D}-4}{4 T_{20}\left(20^{\circ}\right)} . \\
t_{21 .}=\frac{E_{L}-E_{R}}{4 T_{21}\left(20^{\circ}\right)} t_{22}=\frac{\left(E_{L}+E_{R}\right)-\left(E_{U}+E_{D}\right)}{8 T_{22}\left(20^{\circ}\right)} . \\
\quad t_{10}=\frac{E_{U}-E_{D}}{4 i T_{11}\left(20^{\circ}\right)}
\end{gathered}
$$

where $T_{k q}(\theta)$ are analyzing powers for the reaction ${ }^{3} \mathrm{He}(\mathrm{d}, \mathrm{p})$ and $t_{\mathrm{kq}}$ describe the beam polarization in the target coordinate system. The $t_{2 q}$ are functions only of $\tau 20$, the beam tensor polarization in its natural coordinate system, and $\beta$ the spin angle. Therefore, the expressions above can be inverted to give the latter quantities.

If we define a yuanlily A as

$$
\Lambda=\sqrt{6} \frac{t_{20}}{t_{21}}
$$

then $B$ is obtained from

$$
\cos 2 B=\frac{-3 \pm A \sqrt{A^{2}+8}}{A^{2}+9}
$$


This in turn is used to obtain $\tau_{20}$

$$
\tau_{20}=\frac{2 t_{20}}{3 \cos ^{2} B-1} \text {. }
$$

Calibration of the polarimeter consists of measuring the $\mathrm{T}_{2 \mathrm{q}}\left(20^{\circ}\right)$ analyzing powers as a function of incident beam energy for known polarization. The latter is obtained from previously measured $^{2}$ values of $\mathrm{T}_{20}\left(0^{\circ}\right)$. A preliminary calibration is shown in Fig. 11.1-2. We have recently used the polarimeter to calibrate the polarized ion source spin precessor, shown in Sec. 2.1.

During the preliminary calibration run we observed fluctuations. in the gas cell current integration outside statistics and evidence of left-right beam shifts. It has been suggested that the former problem is due to energetic forward-directed electrons from the collimator penetrating the electric suppression. We have therefore included magnetic suppression as shown in Fig. 11.1-1 to eliminate this problem. The left-right shifts are due to an indeterminacy in the beam path through an up-

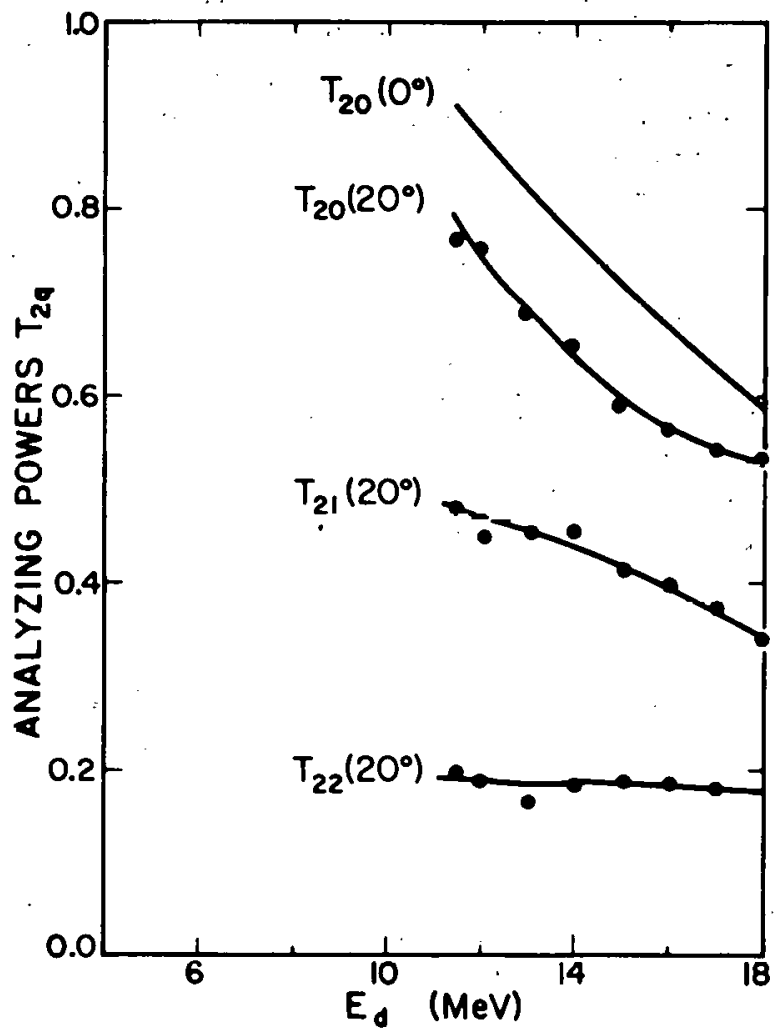

Fig. 11.12. Deuteron polarimeter ten= sor analyzing powers. stream quadrupole lens. Additional collimators should reduce the problem which presently amounts to asymmetry fluctuations on the order of $1 \%$.

1. Nuclear Physics Laboratory Annual Report, University of Washington (1975), p. 91.

2. T.A. Trainor, T.B. Clegg, and P.W. Lisowski, Nucl. Phys. A220, 533 (1974).

11.2 : Elastic Scattering of Polarized Protons from Carbon

T.A. Trainor and W.G. Weitkamp

Measurements of the analyzing power and differential cross section for the elastic scattering of protons from carbon between 11.5 and $18 \mathrm{MeV}$ were reported last year.l Phase shift and resonance parameter analysis of these data has been completed.2 To obtain reasonable fits to the data it was necessary to vary phase shifts with $\ell \leq 4$ and to vary the normalization of the cross section data, giving a total of 20 parameters. Because of this large number of parameters, several sets of parameters were found to give good fits to the data, particularly above $17 \mathrm{MeV}$. To study and possibly reduce these ambiguities, we have taken additional measurements between 17 and $18 \mathrm{MeV}$, increasing the number of data angles from 16 to 27 at each of 10 energies. We have analyzed the effect of the additional data on the shape of the $x^{2}$ surface near the minimum, and have examined the relationship 
between the several sets of phase shifts and the extracted resonance parameters.

The $x^{2}$ surface is a complicated 2l-dimensional surface for a non-linear search problem such as this. One would expect the surface to be roughly parabolic as a function of any one of the parameters near a minimum, with the width of the parabola related to the uncertainty in the parameter. However, several parameters can be correlated so that a change in one can be compensated for by a change in another, without altering the value of $x^{2}$. Normally one can determine such correlations by calculating the error matrix; ${ }^{3}$ in the present case the error matrix has 210 independent elements, so is not of much value. Instead we have chosen a simpler way of characterizing the $x^{2}$ surface near a minimum. We change a given phase shift by a certain amount and vary all other parameters to minimize $x^{2}$. This is repeated for different values of the given phase shift until the curve relating the minimum value of $x^{2}$ to the given phase shift has been traced out. If the given phase shift is well defined by the data, the curve is reasonably parabolic; if a phase shift is highly correlated with other phase shifts, the curve is flat.

This calculation was made for all

phase shifts at $17.87 \mathrm{MeV}$. For phase shifts with $\ell \geq 3$, the curves are roughly parabolic, both for $\delta$, the real part of the phase shift, and $\eta$, which is relaţed to the imaginary part of the phase shift $\mu$ by $n=e^{-2 \mu}$. Using a standard criterion $^{4}$ these curves give typical uncertainties of several degrees for $\delta$ and several times 0.01 for $n$.

For phase shifts with $\ell \leq 2$, the curves were very much broader. Typical examples are shown in Fig. 11.2-1, where $x^{2}$ curves for the $P_{3 / 2}$ and $D_{5 / 2}$ phase shifts are plotted. These curves were calculated with data at 27 angles. It should be pointed out that for all phase shifts the curves calculated with data at 16 angles were insignificantly different from curves calculated with data at 27 angles. In this particular case, it is clear that data at 16 well-distributed angles determines the phase shifts about as well as can be expected.

The $x^{2}$ curve for the $\delta\left(D_{5 / 2}\right)$ phase shift shows the presence of two distinct minima, on the right corresponding to the "preferred" set of phase shifts (indicated by an arrow in Fig. 11.2-1) and on the left corresponding to a second set 
which actually has a smaller $\chi^{2}$ than the preferred set. The preferred set of phase shifts was obtained by constraining the phase shifts at each energy to be reasonably continuous extensions of phase shifts at lower energies. We also calculated a second set of phase shifts by constraining the phase shifts at other energies to be continuous extentions of the phase shifts which produce the left side minimum in the $\delta\left(D_{5 / 2}\right)$ curve. We were not, however, able to extend this second set below $17 \mathrm{MeV}$.

We favor the "preferred" set because of continuity with phases at lower energies. The magnitude of $x^{2}$ is significantly better for the second set: the

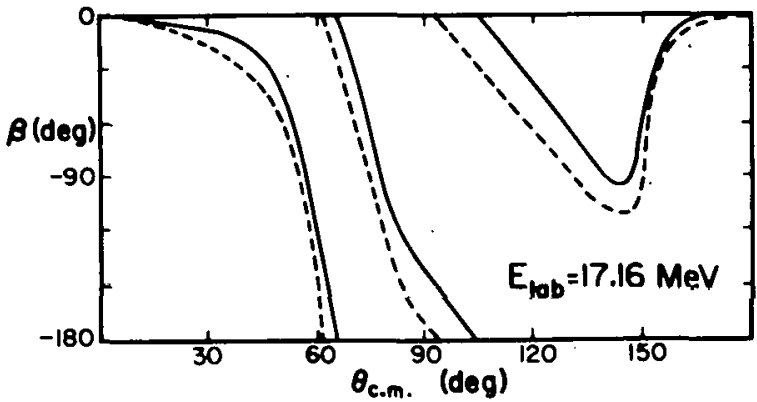

Fig. 11.2-2. Predictions for the spin rotation angle $\beta$. The solid curve is calculated from the preferred set of phase shifts; the dashed line from the second set. average value of $x^{2}$ per datum point is 0.5 for the second set, 0.8 for the preferred set abnve $17 \mathrm{MeV}$. However, the cross section normalization, a variable parameter, deviates from the measurements of Ref. 5 by only an average of only $4 \%$ for the preferred set, but by $11 \%$ for the second set.

It is possible to select between these sets by comparing the predicted value of $\beta$, the spin rotation angle, 6 with a measurement. Figure 11.2-2 shows the predictions for the two sets at $17.16 \mathrm{MeV}$. As may be seen an easily measurable $30^{\circ}$ difference between the two curves is present at a scattering angle of $90^{\circ}$. No measurements of $\beta$ have been made as yet in this energy region.

Both the prcterred oct and the eecond set of phaso shifis show oventice uf resonant behavior as a function of energy in this region. It is of interest to see to what extent the resonance parameters extracted from the two sets differ, particularly because the resonance parameters differ considerably from previously published values. 5

Using the graphical method described in Ref. 7, one can extract rough values for the resonance energy $E_{r}$, the proton partial width $\Gamma_{p}$, the total width $\Gamma$, and the relative phase between the resonance and the background amplitudes $\alpha$. If one plots the scattering matrix element $S=n e^{2 i \delta}$ in the complex plane, one obtains a circle in the vicinity of a resonance, the radius of which is $\Gamma_{\mathrm{p}} / \Gamma$; the other resonance parameters can be extracted from the orientation of the circle in the complex plane.

Such plots are shown in Fig. 11.2-3 for the $P_{1 / 2}$ and $D_{3 / 2}$ matrix elements. The values of $\Gamma_{p} / \Gamma$ are equal for the $P_{1 / 2}$ resonance, and differ by $20 \%$ for the $\mathrm{D}_{3 / 2}$ resonance. This difference is within the uncertainties of the determination. The values of $\alpha$, shown graphically in Fig. 11.2-3, are also in satisfactory agreement. Furthermore, examination of the resonance energy and total widths shows satisfactory agreement. So, we can conclude that the values of the extracted resonance parameters do not depend on the particular phase shift set which is used to represent the non-resonant background scattering. 

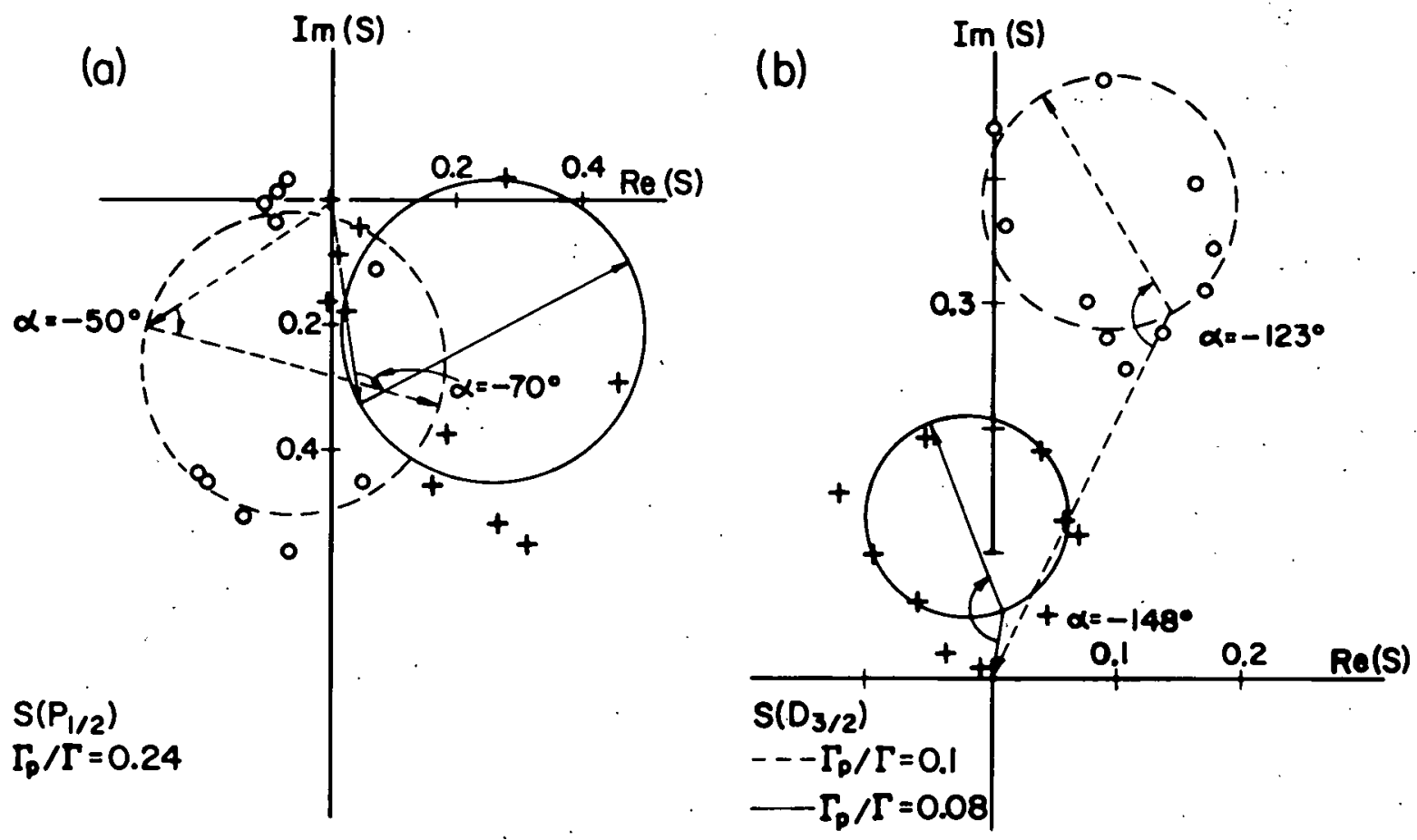

Fig. 11.2-3. Plots of the scattering matrix element $S$ in the region from 17 to $18 \mathrm{MeV}$ for $a)$ the $P_{1 / 2}$ matrix element and $b$ ) the $D_{3 / 2}$ matrix element. Crosses show matrix element values calculated from the preferred set of phase shifts; circles show values calculated from the second set. The solid lines show trajectories calculated from resonance parameters extracted from the preferred set of. phase shifts; dotted lines, from the second set.

1. Nuclear Physics Laboratory Annual Report, University of Washington (1975), p. 97.

2. W.G. Weitkamp, H.O. Meyer, J.S. Dunham, T.A. Trainor and M.P. Baker, Proc. of Fourth Intermational Symposium on Polarization Phenomena in Nuclear Reactions, ed. by W. Gruebler, to be published.

3. P:R. Bevington, Data Reduction and Error Analysis for the Physical Sciences (McGraw-Hill, New York, 1969).

4. D.H. McSherry and S.D. Baker, Phys. Rev. C 1, 888 (1970).

5. M.J. LeVine and P.D. Parker, Phys. Rev. 186, 1021 (1969).

6. B.W. Davies, M.K. Craddock, R.C. Hanna, Z.J. Moroz and L.P. Robertson, Nucl. Phys. A97, 241 (1.967).

7. H.O. Meyer and G.R. Plattner, Nucl. Phys. A199, 413 (1973). 
11.3 Analyzing Power in the ${ }^{207} \mathrm{~Pb}(\overrightarrow{\mathrm{p}}, \mathrm{p}, 0){ }^{207} \mathrm{pb}$ Reaction Near the $3 \mathrm{p}_{1 / 2}$ Isobaric Analog Resonance

N.L. Back, H.C. Bhang, J.G. Cramer, W.G. Lynch, and T.A. Trainor

Recent calculations based on S-matrix theory ${ }^{l}$ predict that the parameters of an isobaric analog resonance (IAR) satisfy the following inequality:

$$
\Gamma \geq 2 \overline{\cos \left(2 \phi_{c}\right)} \sum_{c} \Gamma_{c}
$$

where $\Gamma$ is the total width of the IAR, and $\Gamma_{c}$ and $\phi_{c}$ are the partial width and resonance mixing phase, respectively, of the IAR in the channel $c$. It has been assumed that $\cos \left(2 \phi_{C}\right)$ is approximately the same for all channels, so its average value has been taken outside the sum. A test of this prediction can be made when $\Gamma \leq 2 \sum_{c} \Gamma_{c}$, as this puts a lower limit on $\phi_{c}$. Such a test has been penformed in the case of the ground state IAR in $2{ }^{\prime}{ }^{\prime} \mathrm{Bi} .2$ The results were consistent with a zero resonance mixing phase in the elastic channel $\left(\phi_{R}\right)$; however, the measured partial widths were such that $\Gamma \simeq 2 \sum_{c} \Gamma_{c}$, so the test was inconclusive.

The ground state $\left(\mathrm{O}^{+}\right)$IAR in ${ }^{208} \mathrm{Bi}$ has been studied by Lenz and Temmer ${ }^{3}$ and by Booth and Madsen, ${ }^{4}$ who measured differential cross sections for elastic and inelastic proton scattering on $207 \mathrm{~Pb}$. This resonance is relatively easy to analyze since it is separated from higher-energy resonances by the shell gap. Considering only scattering to the ground state, the $5 / 2^{-}\left(E_{X}=.570 \mathrm{MeV}\right)$ state, and the $3 / 2^{-}\left(E_{\mathrm{X}}=.894 \mathrm{MeV}\right)$ state, we have $\Gamma / 2 \sum_{\mathrm{C}}^{2} \Gamma_{C}=0.86 \pm .15$ (Lenz and Temmer) or $0.81 \pm .06$ (Booth and Madsen). The latter result gives $\overline{2 \phi_{c}} \geq 36^{\circ} \pm 6^{\circ}$. This prediction can be tested by measuring the analyzing power in the vicinity of the resonance.

Analyzing power excitation functions have been obtained at laboratory angles of $105^{\circ}, 120^{\circ}$, and $135^{\circ}$, for proton bombarding energies between 11.0 and $12.1 \mathrm{MeV}$. The target was isotopically enriched $207 \mathrm{~Pb}$, approximately $520 \mu \mathrm{g} / \mathrm{cm}^{2}$ thick, on a $20 \mu \mathrm{g} / \mathrm{cm}^{2}$ carbon backing. Left-right asymmetries were measured with three pairs of $\mathrm{Si}(\mathrm{Li})$ detectors with acceptance angles of $\pm 0.9^{\circ}$ or less. The beam polarization was continuously monitored by measuring the left-right asymmetry of protons elastically scattered from $4 \mathrm{He}$ at $112^{\circ}$. Each measurement was made twice, once with spin up and once with spin down, in order to compensate for instrumental asymmetries.

A typical on-resonance spectrum is shown in Fig. 11.3-1. The off-resonance spectra are similar except for the inelastic peaks, which can barely be seen above the background. For the elastic peak, the statistical uncertainty is $0.5 \%$ or better, including the errors introduced in background subtraction. The target thickness at this energy is about $8 \mathrm{keV}$.

The excitation functions obtained are shown in Fig. 11.3-2. The structure due to the resonance at $11.53 \mathrm{MeV}$ is clearly seen at all three angles. The curves are calculations of the analyzing power, with the background described by the optical model and the resonance by a Breit-Wigner term. The optical model parameters were taken from an analysis of proton elastic scattering on $207 \mathrm{~Pb}$ at 


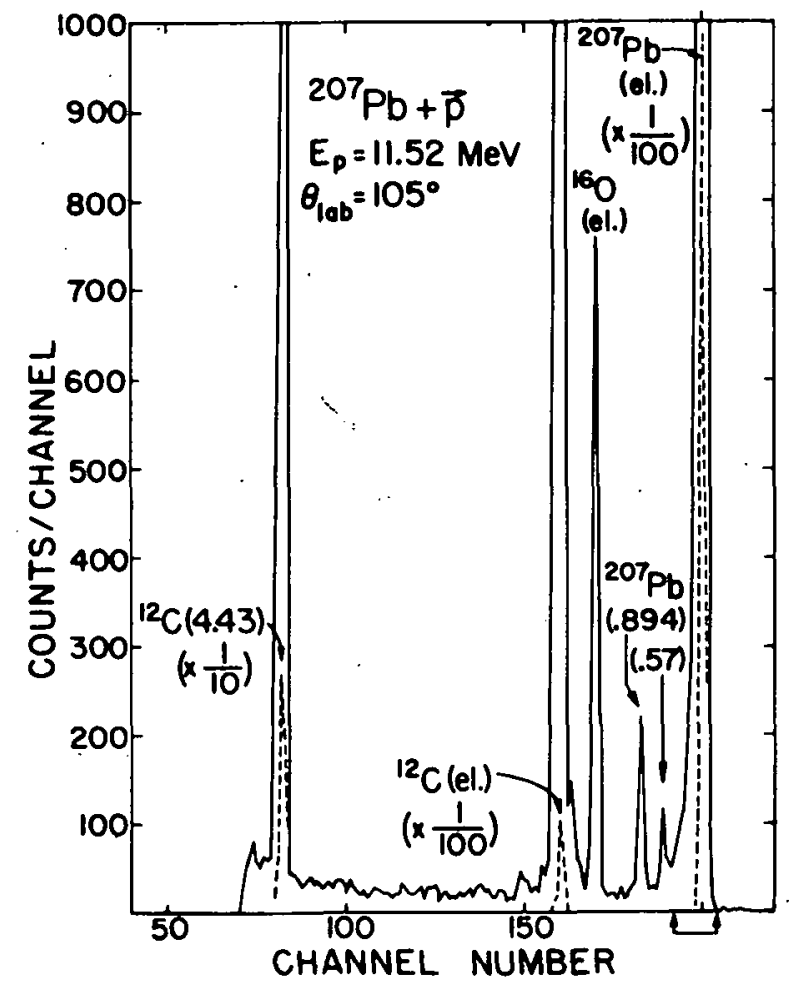

Fig. 11.3-1. Pulse-height spectrum of $207 \mathrm{~Pb}(\overrightarrow{\mathrm{p}}, \mathrm{p}){ }^{207} \mathrm{~Pb}$ on resonance. The bracket shows the channels used for peak integration.

$12.98 \mathrm{MeV}$ by Rathmell and Haeberli; 5 the real and imaginary potentials are given linear energy dependences as suggested by Becchetti and Greenlees. 6 The effects of lie $5^{-}$IAR at $E_{c . m}=14.70 \mathrm{MeV}$ are also included, with parameters determined by Ramavataram et al. 7 The total width of the $0^{+}$IAR was taken from the analysis of inelastic scattering by Booth and Madsen. The other resonance parameters were varied to obtain the best fit to the data, although no formal fitting routine was used. The parameters used are shown in Table 1l.3-1. Two curves are shown in Fig. 11.3-2, one for $\phi_{R}=0^{\circ}$ and the other for $\phi_{R}=7.5^{\circ}$. The reduced $x^{2}$ is 7.13 at $0^{n}$ and 4.00 at $7.5^{\circ}$.

Because of the poor quality of the fits, especially in the tail region, it is not yet possible to make any definite statements about the resonance mixing phase. It appears that the optical model parameters that were used do not adequately describe the background and will have to be modified. In this regard, it is worth noting that (a) Ref. 5 did not include the effects of the resonances, even though such effects are not insignificant, and (b) the fit to the data of 
Table 11.3-1., Resonance parameters used to fit $207 \mathrm{~Pb}(\overrightarrow{\mathrm{p}}, \mathrm{po}){ }^{207} \mathrm{~Pb}$ excitation functions.

$\begin{array}{cccc}\mathrm{E}_{\mathrm{R}}(\mathrm{MeV}) & \Gamma_{\mathrm{P}}(\mathrm{keV}) & \Gamma(\mathrm{keV}) & \phi_{\mathrm{R}}(\mathrm{deg}) \\ 11.475 & 56 & 231 & 7.5 \\ 14.70 & 17 & 196 & 15\end{array}$

Ref. 5 was very insensitive to the spinorbit geometry (i.e., $r_{s .0}$, and $a_{s . o .}$ ). To aid in the search for new background parameters, angular distributions of the analyzing power have been measured at $E_{p}=10 \mathrm{MeV}$ and $13 \mathrm{MeV}$. The data were obtained using the same experimental procedure as for the excitation function measurements. Due to the difficulty of separating the carbon and oxygen peaks from the lead peak, the forward-angle points are subject to some additional error. The angular distributions are shown in Fig. 11.3-3. The curves shown

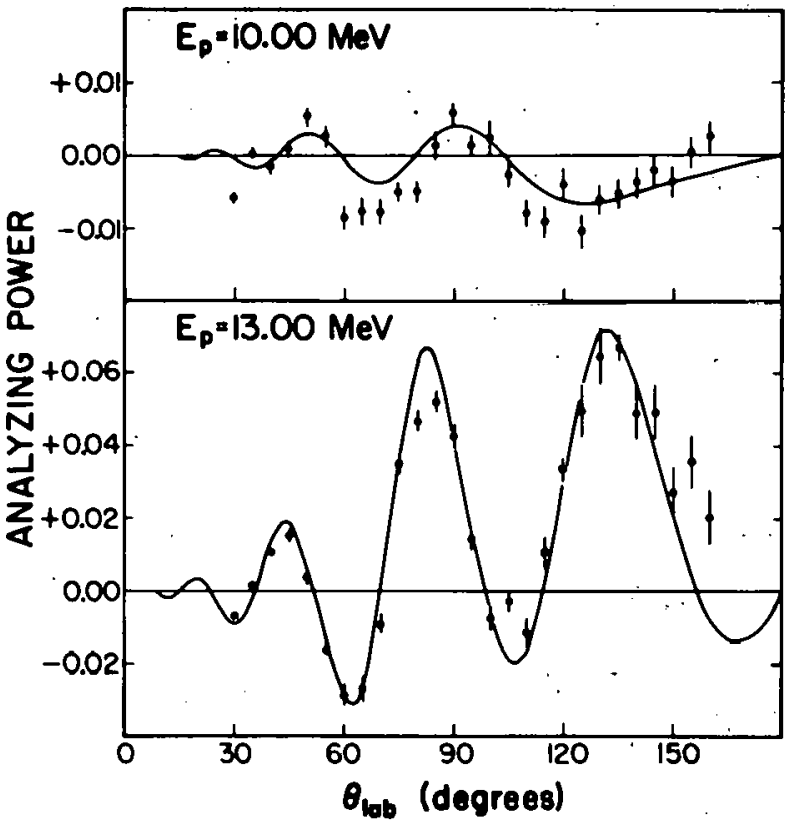
are obtained using the optical model with Breit-Wigner terms for the resonances, as before. The parameters used are the same as in Fig. 11.3-2 (with $\phi_{R}=7.5^{\circ}$ ).

In conclusion, until a better description of the background can be obtained, it will not be possible to determine the resonance mixing phase accurately. However, at this point it appears unlikely that the inequality $2 \phi_{\mathrm{R}} \geq 36^{\circ}$ can be satisfied.

1. P. von Brentano in Proceedings of the Europhysics Study Conference on Intermediate Processes in Nuclear Reactions, ed."N, Cindro (SpringerVerlag, New York, 1973); p. 267.

2. M.P. Baker, T.A. Trainor, J.S. Blair, J.G. Cramer, and W.G. Weitkamp, in Proceedings of the Fourth Intemational Symposizm on Polarization Phenomona in Nualoar Roactions (to be fullisliel).

3. G.H. Lenz and G.M. Temmer, Nucl. Phys. A112, 625 (1968).

4. E.C. Booth and B.S. Madsen, Nucl. Phys. A206, 293 (1973).

5. R.D. Rathmell and W. Haeberli, Nucl. Phys. A178, 458 (1972).

6. F.D. Becchetti and G.W. Greenlees, Phys. Rev. 182, 1190 (1969).

7. K. Ramavataram, S. Ramavataram, W.G. Davies, A.J. Ferguson, and J.S. Fisher, Phys. Rev. C 10, 632 (1974). 
11.4 Analyzing Powers for the Continuum Portions of Particle Emission Spectra

H. Bhang, I. Halpern, K-L Liu, W. Lynch, B. Tsang, and T.A. Trainor.

We have recently begun a program to search for polarization effects in the continuum portions of the spectra of emitted particles. In particular we have been measuring analyzing powers in various portions of alpha-particle and proton spectra when medium-weight targets are bombarded with polarized protons. In the past analyzing powers have been measured mainly for low-lying discrete levels in residual spectra. The continuum portions of emission spectra contain evaporated particles and particles emitted in direct reactions to residual levels that are unresolvable. The analyzing power for the evaporated particles is expected to vanish in the simplest models for evaporation and is expected to be small for the direct emissions to superposed residual levels. We believe that the measurement of these (small) analyzing powers is likely to shed some useful light on the mechanisms responsible for these emissions. This type of experiment is made possible by the recent refinement of polarization techniques which allow reliable measurement of analyzing powers of order $10^{-3}$ or smaller.

We have chosen for initial study targets of ${ }^{58} \mathrm{Ni},{ }^{63} \mathrm{Cu}$ and ${ }^{64} \mathrm{Zn}$. This mass region provides a compromise between relatively high charged-particle yield at tandem energies and a sufficient density of states to allow statistical treatment over a broad range of excitation energies. Tanget thicknesses are typically $600 \mathrm{\mu g} / \mathrm{cm}^{2}$. This figure insures reasonable count/rates at all angles, yet provides adequate energy resolution for lowest energy emitted alpha particles.

Emitted protons and alpha particles were detected by two particleidentification telescopes placed at symmetric angles. The telescope consisted of $20 \mu \Delta \mathrm{E}$ detectors and $2-3 \mathrm{~mm} \mathrm{E}$ detectors. Signals from left and right detectors wcre routed into different computer storage areas depending on whether the beam spin direction was up or down with respect to the scattering plane. The route signal was obtained from digital circuitry which reverses the spin direction at the polarized source at a rate of $1 \mathrm{hz} .1$ This fast-flip procedure reduces instrumental effects due to spin-correlated beam steering to less than $10^{-3}$ in $A_{y}$. A check on instrumental effects comes from "second-chance" evaporated protons at the low-energy end of the proton spectrum. These protons are expected to show zero analyzing power.

The polarized ion source produced target proton currents of 60-120 nA. Beam current was adjusted to a count-rate limited value at each scattering angle. Polarization was about 0.70 as monitored by a helium polarimeter.

The on-line PID data-açuisition program sorted charged-particle events corresponding to left and right detectors and incident proton spin up or down. The program also performed on-line calculations of $\mathrm{A}_{y}$, instrumental asymmetries, charge asymmetries, and total yields for each of several regions light-penned in the various spectra.

Typical spectra corresponding to a $60^{\circ}$ lab scattering angle at 14 and 18 $\mathrm{MeV}$ incident proton energies are shown in Fig. 11.4-1. The lower cutoff is about $1 \mathrm{MeV}$ for the proton spectra and $2.5 \mathrm{MeV}$ for the alpha spectra. Several discrete peaks in the evaporation region are identified as inelastic groups from ${ }^{1} \mathrm{H},{ }^{12} \mathrm{C}$, 

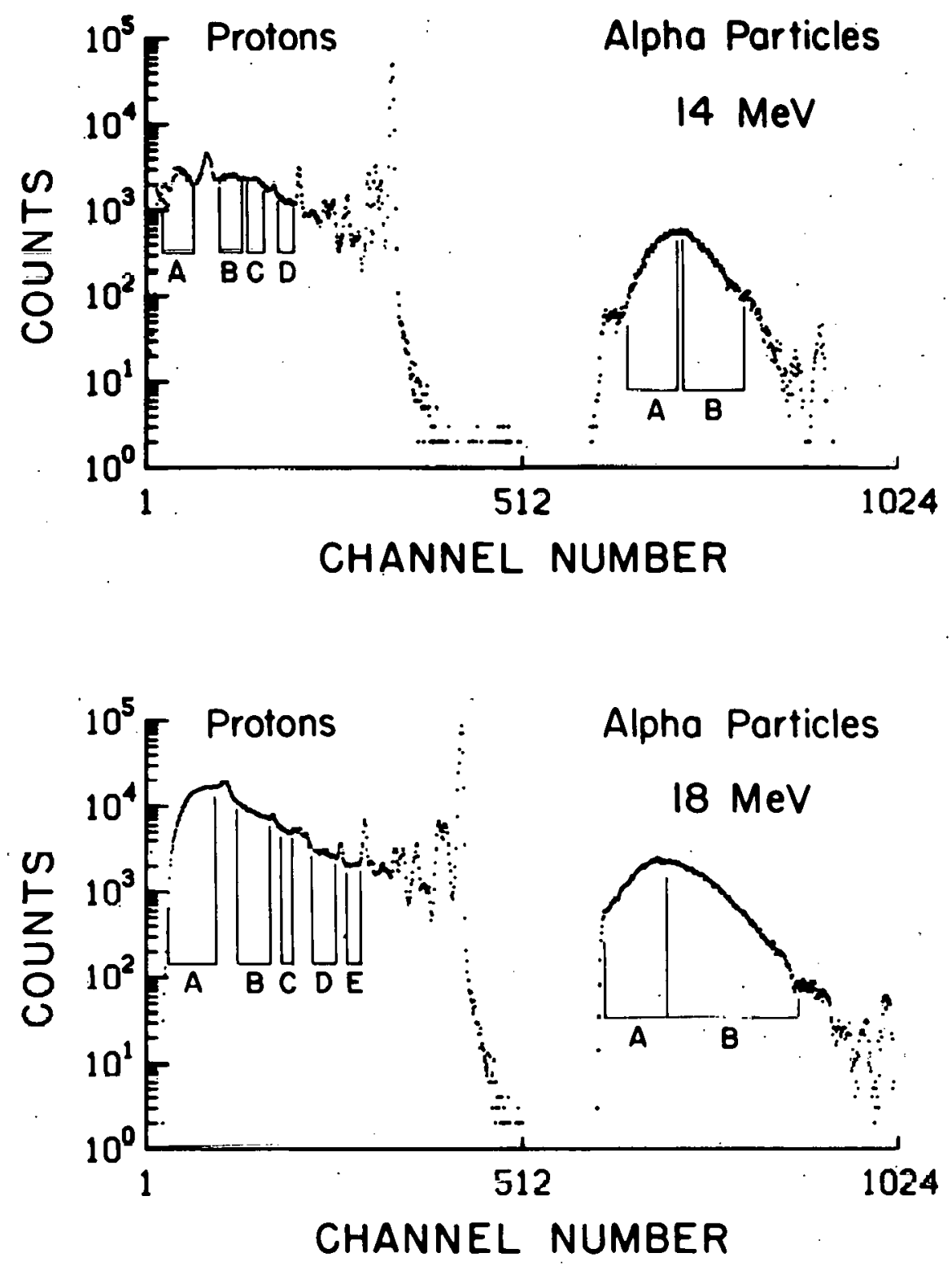

Fig. 11.4-1. Proton and alpha-particle emission spectra at $\theta_{1 a b}=60^{\circ}$ for 14 and $18 \mathrm{MeV}$ polarized protons incident on ${ }^{63} \mathrm{Cu}$. 
and ${ }^{16} 0$ contamination of the target. The analyzing power for each contaminant group has been measured with the same experimental setup described above for purposes of off-line correction.

The energy calibration for each telescope was monitored periodically by proton scattering from a $1 \mu \mathrm{g} / \mathrm{cm}^{2}$ carbon target. Data at each energy and angle were collected in four or eight separate time intervals in order to allow detection of any time-dependent systematic errors. These quartets or octets of data were later combined when no difficulties were encountered. Preliminary results for the analyzing powers for regions labeled in Fig. 11.4-1 are given in Table 11.4-1.

Table 11.4-1. Analyzing powers for selected regions of proton and alphaparticle spectra (see Fig. 11.4-1).

$$
{ }^{63} \mathrm{Cu}\left(\mathrm{p}, \mathrm{p}^{\prime}\right)^{63} \mathrm{Cu} * \theta_{\mathrm{lab}}=60^{\circ}
$$

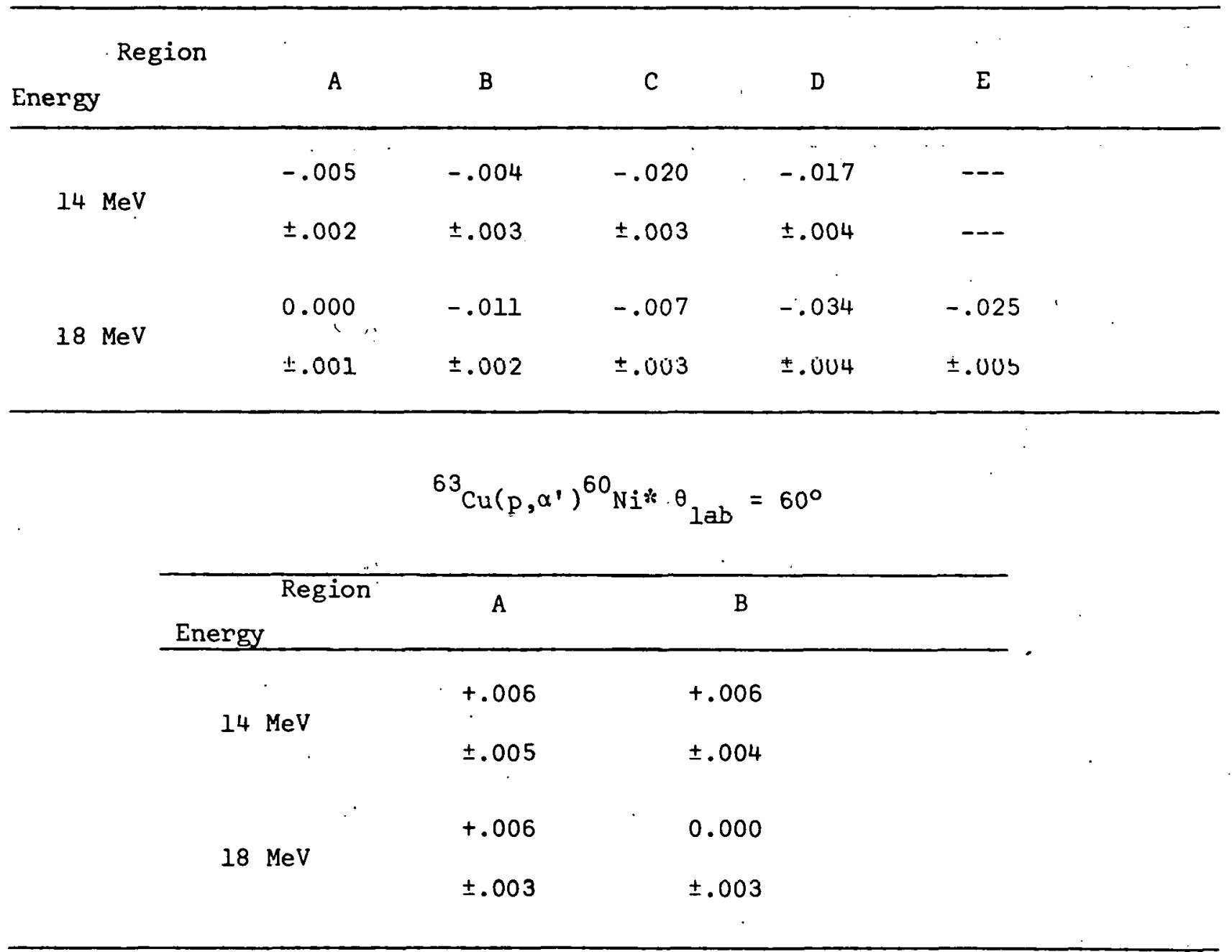


It can be seen from these results that there is a trend toward negative analyzing powers for the proton spectrum and positive or zero analyzing powers for the alpha-particle spectrum in the evaporation region at $60^{\circ}$. This is to be contrasted with results at $120^{\circ}$ where both alpha particle and proton spectra analyzing powers are now predominantly positive or zero over the evaporation region. These figures are very preliminary and do not include corrections for target impurities. However, an estimate of impurity contributions for the regions covered in the table indicates that corresponding errors should be less than $10^{-3}$ in $A_{y}$ for all cases.

We are presently considering several alternative schemes for stripping impurity peaks from the spectra. This operation must be performed very carefully so as not to introduce false asymmetries as a result of the stripping procedure. Before we can deal with all portions of the continuum spectra; it will be necessary to carry out the stripping procedure in a satisfactory way.

To summarize: We have seen small but finite analyzing powers in the higher energy portions (and perhaps even in the evaporation portions) of the continuum emission spectra in bombardments with polarized protons. We hope to improve the accuracy of these measurements and to formulate models that permit us to account for the magnitudes and the angular distributions of the analyzing powers we see. 


\section{HEAVY ION ELASTIC AND INELASTIC SCATTERING}

-1.2 Evidence for Shallow Strongly Absorbing Heavy-Ion Optical Potentials J.G. Cramer, R.M. DeVries ${ }^{\dagger}$, D.A. Goldberg ${ }^{\dagger \dagger}$, and M.S. Zisman ${ }^{\xi}$

Over the past several years we have conducted a detailed investigation of the elastic scattering of ${ }^{16} \mathrm{O}$ from ${ }^{28} \mathrm{Si}$ and similar targets over a range of energies. We have concluded from this study that there are three principal regions of the nuclear potential which are probed by this type of elastic scattering analysis: (a) the extreme tail region of the potential, which is probed by elastic scattering performed very near the Coulomb barrier; (b) the strong absorption radius (or more quantitatively, the semi-classical turning radius of the grazing partial wave) which is probed by scattering at essentially all bombarding energies between the Coulomb barrier and about $100 \mathrm{MeV}$; and (c) the surface region and "outer. interior" which are probed by scattering at bombarding energies above about $100 \mathrm{MeV}$, where enough energy is available to observe nuclear "rainbow" scattering.

Thus if the nuclear potential is assumed to be independent of energy, it can be mapped by simultaneously fitting data of types (a), (b), and (c), as described above. In an effort to enlarge the information from the interior region, we have in a collaborative experiment studied the scattering of 160 from $28 \mathrm{Si}$ at a laboratory bombarding energy of $215.2 \mathrm{MeV}$, using the 160 beam from the Berkeley 88" cyclotron. These data, together with data collected at lower energies ${ }^{1}$ were fitted with a global analysis, requiring the same energyindependent 6-parameter Woods-Saxon potential to fit both high and low energy data. The results are shown in Fig. 12.1-1. The potentials used in generating the curves in Fig. 12.1 are given in Table 12.1-1.

Table 12.1-1. Derived Optical Model Potentials

\begin{tabular}{|c|c|c|c|c|c|c|c|c|}
\hline Label & $v_{0}$ & $r_{0}$ & $a_{0}$ & $\begin{array}{c}\mathrm{W}_{0} \\
\text { (volume) }\end{array}$ & $r_{I}$ & $a_{I}$ & $\begin{array}{c}x^{2} / \mathrm{N} \\
(215.2 \mathrm{MeV})\end{array}$ & $\begin{array}{c}\chi^{2} / \mathrm{N} \\
(38.0 \mathrm{MeV})\end{array}$ \\
\hline E18 & 10 & 1.35 & .618 & 23.4 & 1.23 & .552 & 4.9 & 1.2 \\
\hline A23 & 100 & .932 & .797 & 165 & .890 & .764 & 8.6 & 15 \\
\hline S75 & 100 & 1.06 & .640 & 42.0 & 1.06 & .640 & $1.3 \times 10^{5}$ & 2.0 \\
\hline $\begin{array}{l}\mathrm{R}=r( \\
\mathrm{E}_{16_{0}}=\end{array}$ & $\begin{array}{l}6^{1 / 3} \\
81 \mathrm{Me}\end{array}$ & $\begin{array}{l}28^{1 / 3} \\
\text { only. }\end{array}$ & $\mathrm{r}_{\mathrm{Co}}$ & $\mathrm{mb}=1.0$. & \multicolumn{4}{|c|}{ Potential $\mathrm{S} 75^{\mathrm{l})}$ is a fit to the data for } \\
\hline
\end{tabular}

There is a clear preference for the shallow potentials in this analysis. While it is possible to find deeper potentials which can fit either the low energy data or the high energy data, we have found no deep potential which is capable of fitting both low and high energy data at the same time. This fit is "unique" 

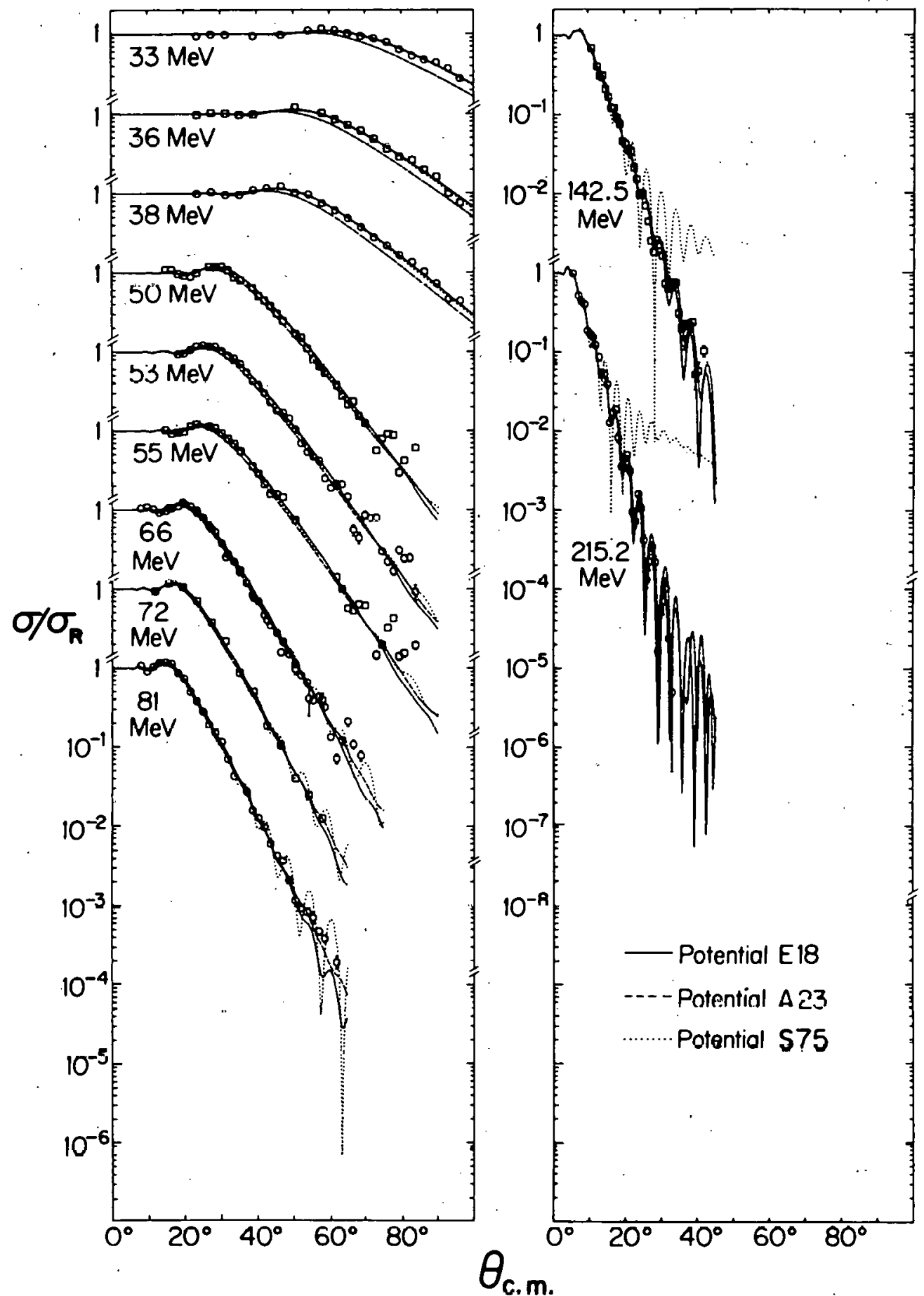

Fig. 12.1-1. Elastic ${ }^{16} \mathrm{O}+{ }^{28} \mathrm{Si}$ scattering at the labeled incident energies. The lines are optical model calculations using the parameter sets indicated and listed in Table 12.1-1. 
only in the context of energy-independent Woods-Saxon potentials.

It should perhaps be mentioned that Satchler has recently analyzed the data set shown in Fig. 12.1-1, from the point of view of the folding model. ${ }^{2} \mathrm{He}$ has started with a realistic nucleon-nucleon potential and obtained an effective real potential with a double-folding procedure while using an empirical WoodsSaxon imaginary potential. It was found to be necessary to renormalize the real potential and to use an energy-dependent geometry for the imaginary potential, but with these modifications, it was possible to obtain quite a good fit to these data. Thus it is clear that deeper non-Woods-Saxon energy-dependent potentials are quite capable of fitting the data set.

The results presented here pose an interesting question: Does the preference which has been found for a $10 \mathrm{MeV}$ real well depth really mean that overlapping $16 \mathrm{O}$ and $28 \mathrm{Ni}$ nuclei have such a weak interaction? Even at our highest incident energies., "notch-perturbation" tests of potential sensitivity indicate no contributions to the scattering from separation distances of less than $5.5 \mathrm{fm}^{3}$ Thus the derived $V=10 \mathrm{MeV}$. value only indicates the potentials' behavior in the nuclear surface region in terms of a Woods-Saxon shape and does not preclude the existence of a soft repulsive-core, for example. Moreover, the energy required to separate ${ }^{44} \mathrm{Ti}$ into $16 \mathrm{O}+28 \mathrm{Si}$ is $11.48 \mathrm{MeV}$. Therefore, in the zero incident energy limit, the bound-state potential for this system would be considerably in excess of $10 \mathrm{MeV}$. Even with these qualifications, the derived $\mathrm{V}=10 \mathrm{MeV}$ value for 160 ions represents a striking departure from the monotonic increase in real well depths found for light-ions, i.e., $50 \mathrm{MeV}$ for nucleons, 80-100 MeV for deuterons, 110-130 MeV for alphas.

It is clear that more theoretical work on the potentials appropriate to heavy-ion interactions is indicated. It would also appear that more experimental data is needed on the elastic scattering at high and low energies of projectiles in the mass region $4<\mathrm{A}<16$ so that optical potentials in this critical transition region can be determined.

+ Department of Physics, University of Rochester, Rochester, N.Y.

t† Department of Yhysics, University of Maryland, Cullege Park, MD.

$\S \quad$ Lawrence Berkeley Laboratory, University of California, Berkeley, CA.

1. Nuclear Physics Laboratory Annual Report, University of Washington (1975), p. 128 .

2. G.R. Satchler, private communication.

3. See Sec. 12.3 of this report.

12.2 Application of the Austern-Blair Theory to Nuclear-Coulomb Interference in Heavy lon Inelástic Scattering

John G. Cramer and C.K. Gelbke

One of the more striking observations which has emerged from recent studies of heavy ion reactions is that of the pronounced interference minima in inelastic scattering differential cross sections and excitation functions arising from the destructive interference of amplitudes for excitations by the Coulomb 
and nuclear fields. It has been suggested that measurements in the region of nuclear-Coulomb interference should have particular sensitivities to the details of the nuclear potential.1

On the other hand, Austern and Blair showed over a decade ago that inelastic scattering amplitudes for strongly absorbed particles are closely related to the elastic scattering amplitudes and implicitly should be no more sensitive to potential details than is elastic scattering. ${ }^{2}$ Thus there is a conflict between these two points of view which can be resolved by calculating inelastic scattering amplitudes in the Coulomb-nuclear interference region using the Austem-Blair approximation and comparing the results with those obtained from more exact DWBA calculations. This is what we have done.

Actually, the Auotern Blair apploximaliun is a serles of related approxlmations, and so one must be more specific as to what approximations are actually being employed. We have investigated a number of levels of approximation which will not be discussed here, and have found that the most satisfactory version of the Austern-Blair approximation involves the following procedure: (1) calculate the elastic scattering reflection coefficients $n_{\ell}$ for both the entrance and exit channels (i.e., at the entrance and exit channel.c.M. energies), (2) numerically differentiate the reflection coefficients with respect to $\ell$, using the approximate relation

$$
\mathrm{d} n_{\ell} / \mathrm{d} \ell \simeq\left\{3 n_{\ell}-4 n_{\ell-1}+n_{\ell-2}\right\} / 2
$$

(3) This derivative is then used to obtain an approximate value of the diagonal radial integrals for inelastic scattering:

$$
I_{\ell}^{(A B)}(E) \simeq \frac{i E}{2 \ell+I} \sqrt{n^{2}+\ell(\ell+I)} \frac{\partial \eta_{\ell}(E)}{\partial \ell}
$$

where $E$ is the C.M. energy and $n$ is the Sommerfeld parameter $n=Z Z^{\prime} e^{2} / h v$. (4) The off diagonal nuclear radial integrals $J \mathbb{V}_{\ell}$, are approximated in two ways and the results compared. Approximation $A B$ (1) uses the diagonal integral of the average angular momentum é:

$$
J_{\ell_{f}, l_{i}}^{(I)} \equiv \delta_{\lambda} I_{\bar{\ell}}\left(E_{i}\right)
$$

where $E_{i}$ is the C.M. energy in the entrance channel and $\bar{l}=\left(\ell_{i}+\ell_{f}\right) / 2$. This This has the advantage of requiring an optical model calculation only at the entrance channel, but at the sacrifice of arnurasy. Approximation $\Lambda B$ (2) was suggested by Hahne ${ }^{3}$ and gives better accuracy because it treats the entrance and exit channels symmetrically:

$$
J_{\ell_{f}, \ell_{i}}^{(2)} \equiv \delta_{\lambda} \sqrt{I_{\ell_{f}}\left(E_{f}\right) I_{\ell_{i}}\left(E_{i}\right)}
$$

In both of the above expressions, $\delta,=\beta_{\lambda} R_{c}$ where $\beta_{\lambda}$ is the deformation parameter for multipolarity $\lambda$ and $R_{C}$ is the charge radius of the nucleus. 
The Coulomb radial integrals $J_{l_{f}}^{C} \ell_{i}$ cannot be calculated with the AusternBlair approximation, and so an alternative procedure was used. Pure Coulomb radial integrals $J l_{f}^{C}, l_{i}$ were obtained with the fast recursion technique used in the program of Samuels and Smilansky.4 For the higher partial waves where the $n_{\ell} \approx 1$ to better than one part in $10^{5}$, i.e., where the non-Coulomb distortions were very small, the Coulomb radial integrals were taken as equal to the pure Coulomb values. For lower partial waves, one of two methods was employed: (1) the Sopkovitch approximation ${ }^{5}$ was employed:

$$
J_{\ell_{f}, \ell_{i}}^{C} \simeq \sqrt{n_{\ell_{f}}^{n_{l} \ell_{i}}} J_{\ell_{f}, \ell_{i}}^{P C} \equiv J_{\ell_{f}, \ell_{i}}^{C-S}
$$

and (2) the radial integrals were numerically calculated by integrating the form factor for coulomb excitation with the distorted waves in the entrance and exit channel, i.e., using a DWBA calculation for the nuclear-distorted Coulomb excitation part of the calculation only. The latter is more accurate, but it is also more time consuming and begs the question of whether the nuclear distortions lead to extra potential sensitivities in the inelastic channel. From a comparison of calculations using these two methods, as will be discussed below, there are no apparent "extra sensitivities" arising from the nuclear distortions.

We have chosen the inelastic scattering of ${ }^{16} \mathrm{O}$ from ${ }^{56} \mathrm{Fe}$ at $E_{\mathrm{lab}}=43 \mathrm{MeV}$ near the Coulomb barrier as a case for the investigation of the above approximations. Figure 12.2-1 shows a comparison of a DWBA calculation with the two approximations given in Eqs. (3) and (4). As can be seen, the latter which is labeled $\mathrm{AB}(2)$ is clearly better and indeed is almost indistinguishable from the DWBA calculation. In all of these calculations, the Coulomb radial integrals were handled by means of method (2) above. In Fig. 12.2-2 we compare the results using Eq. (5), the Sopkovitch approximation, with the results of using numerically integrated Coulomb excitation radial integrals for the lower partial waves. As is apparent from the figure, the Sopkovitch approximation labeled $A B$ (2)-C works surprisingly well in reproducing the more accurage calculations.

Finally, we have used the $A B(2)$ approximation to calculate excitation functions for the same reaction at backward angles. These are shown in Fig. 122-3, and it is apparent that the approximation works just as well for excitation functions as for angular distributions, with really excellent agreement.

These calculations have shown that the Austern-Blair approximation works surprisingly well for heavy ion inelastic scattering in the region of Coulomb-nuclear' interference and may provice a very powerful auxilliary

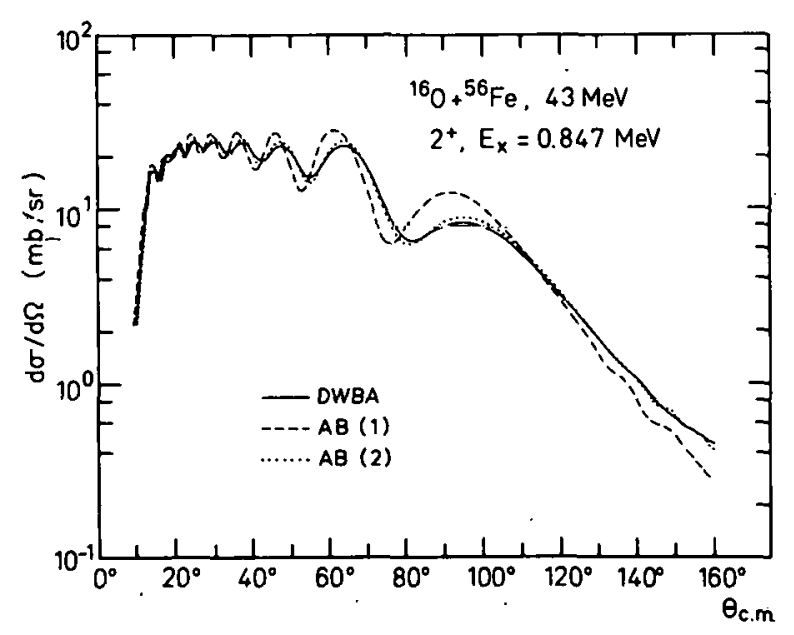

Fig. 12.2-1. Comparison of DWBA calculations and Austern-Blair approximations $A B(1)$ and $A B(2)$ for the inelastic scattering of 160 from $56 \mathrm{Fe}$ at $43 \mathrm{MeV}$ laboratory bombarding energy. 

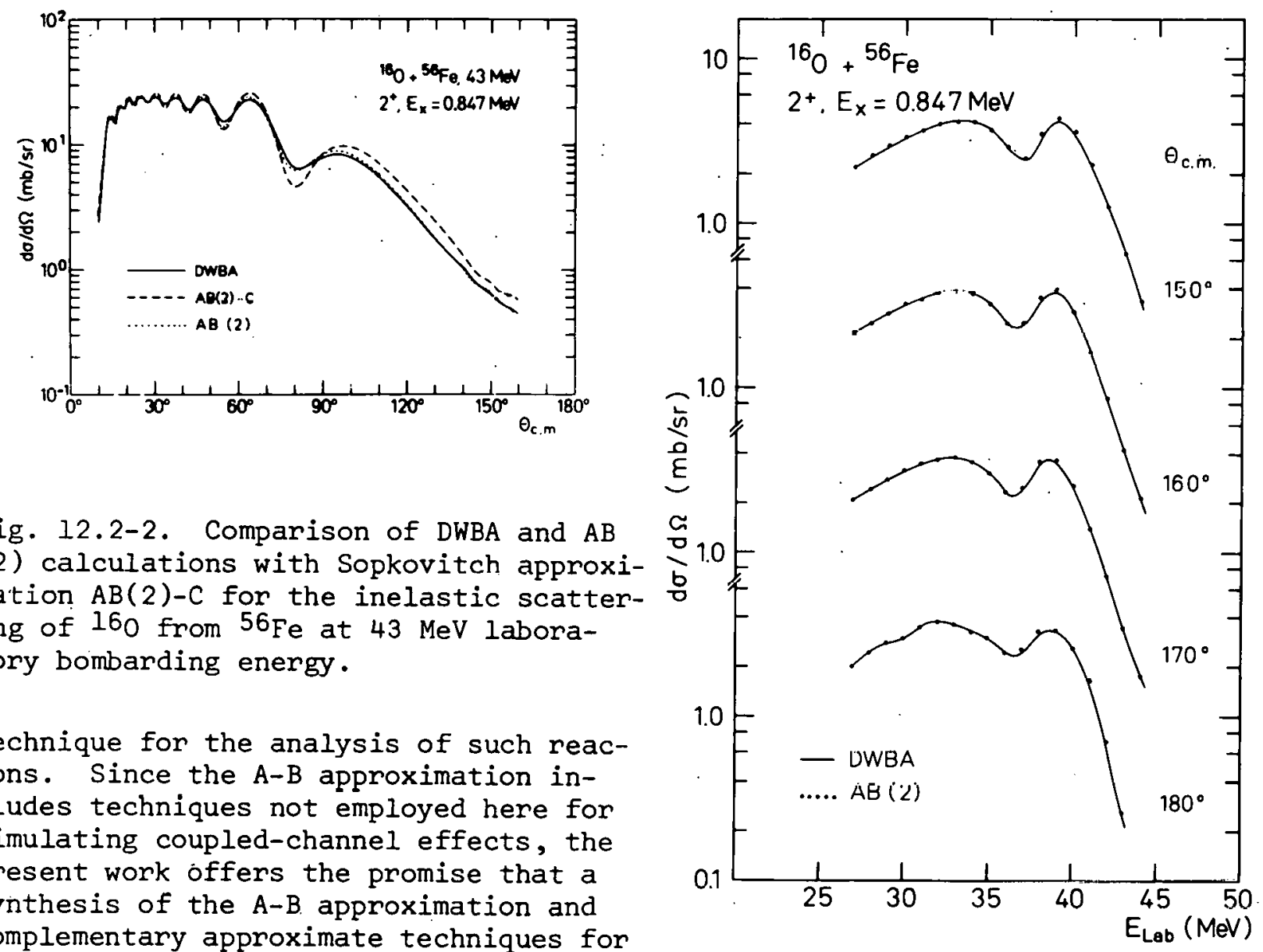

Fig. 12.2-2. Comparison of DWBA and $A B$ (2) calculations with Sopkovitch approximation $A B(2)-C$ for the inelastic scattering of 160 from $56 \mathrm{Fe}$ at $43 \mathrm{MeV}$ laboratory bombarding energy.

technique for the analysis of such reacions. Since the A-B approximation includes techniques not employed here for simulating coupled-channel effects, the present work offers the promise that a synthesis of the A-B approximation and complementary approximate techniques for the treatment of pure Coulomb-excitation coupled-channel calculations could provide an even more powerful tool for reaction analysis.

Another result of these calcula-

Fig. 12.2-3. Comparison of DWBA and $A B$ (2) calculations for excitation functions of the inelastic scattering of 160 from $56 \mathrm{~F}$ 'e at venter of mass angles of $150^{\circ}$, $160^{\circ}, 170^{\circ}$, and $180^{\circ}$. tions is to provide clear evidence that there is very little additional information to be gained from the analysis of Coulomb-nuclear interference in inelastic scattering (aside from $B(E 2)$ values) that is not implicit in the corresponding elastic scattering data and analysis. (A longer version of this work has recently been submitted for publication in Physical Review.)

1. P.R. Chriștensen, I. Chernov, E.E. Gross, R. Stokstad, and F. Videbaek, Nucl. Phys. A207, 433 (1973).

2. N. Austern and J.S. Blair, Ann. Phys. 33, 15 (1965).

3. F.J.W. Hahne, Nucl. Phys. 80, 113 (1966).

4. M. Samuel and U. Smilansky, Comp. Phys. Comm. 2, 455 (1971).

5. N.J. Sopkovitch, Nuovo Cimento 61, 186 (1962); see also J.S. Blair, Ann. Phys. 66, 721 (1971). 
12.3 Notch Perturbation Tests of the Sensitivity of the Heavy Ion Optical Model to the Details of the Nuclear Potential

John G. Cramer

Perhaps the most persistent problem in the analysis of heavy ion scattering and reactions is that of potential sensitivity. While there is abundant qualitative evidence that only a very limited region of an interaction potential plays a role in determining the scattering and reaction cross sections; there has been very little quantitative study of this very important aspect of nuclear reactions. As a result, there is universal confusion over what is a "good". heavy ion optical potential, and indeed many have come to look with distaste on any studies of optical potentials as one of the lower forms of "computerology".

The present work is an effort to put this question of sensitivities on a more quantitative basis by providing a procedure by means of which a "sensitivity function" can be generated for a given potential in a given reaction or scattering situation. The basic approach is to introduce a radial perturbation into the potential in a localized radial region and to observe its effect on the predicted cross sections. It is asserted that in a region where such a perturbation has only a weak effect on the cross section there is weak sensitivity, and where the effect on the cross section is strong there is strong sensitivity. Since such cross sections are ultimately to be compared to experimental data, it seems more meaningful to compare the perturbed cross sections to data than to an unperturbed cross section calculation. We have therefore used the $x^{2}$ value of the fit to data as a measure of the effect of the perturbation of the potential on the cross section.

Figure 12.3-1 shows the kind of perturbation used. The perturbed potential $v_{p}(r)$ has the form:

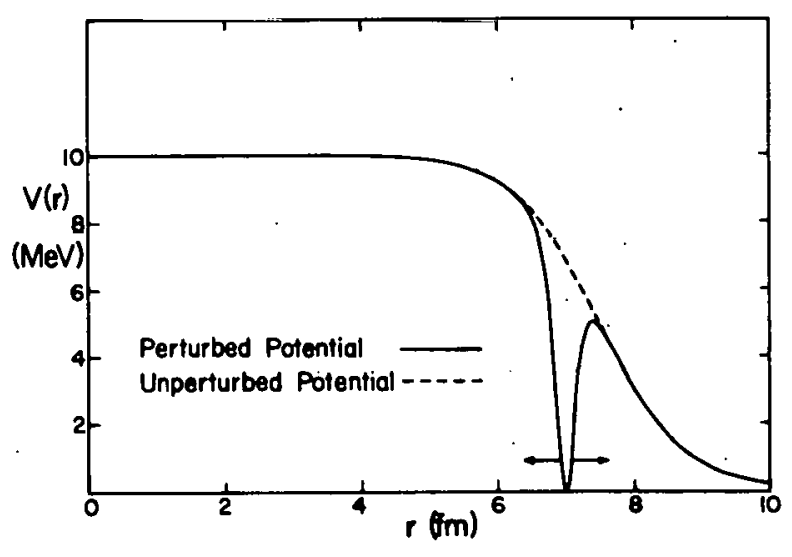

$V_{p}(r)=V_{0}(r)\left[1-4 f\left(R^{\prime}, a^{\prime} ; r\right)\left(1-f\left(R^{\prime}, a^{\prime} ; r\right)\right)\right]$

whene.

$$
f\left(R^{\prime}, a^{\prime} ; r\right)=\left[1+\exp \left(\left(r-R^{\prime}\right) / a^{\prime}\right)\right]^{-1}
$$

and $V_{0}(r)$ is the unperturbed potential. As shown, this multiplicative factor has the effect of cutting a "notch" out of the potential and reducing it to zero in a localized region. The position of the center of this notch is the $R^{\prime}$ parameter and the FWHM of the notch is the diffuseness parameter a' multiplied by $4 \ln (1+\sqrt{2})$, i.e., FWHM $=3.52549 \mathrm{a}^{\prime}$. This perturbation function may be applied to either the real on the imaginary potential, so that the sensitivi-

Fig. 12.3-1. Comparison of perturbed and unperturbed potential. Potential used is El8 with $V=10 \mathrm{MeV}, R_{0}=7.50 \mathrm{fm}$, and $a_{0}=0.618 \mathrm{fm}$. The parameters of the notch function are $R^{\prime}=7.0 \mathrm{fm}$ and $a^{\prime}=0.10 \mathrm{fm}$. 
ties to each may be examined separately. As should be apparent, the width of the notch will determine the "strength" of the perturbation, with wide notches producing gross alterations in the cross section and very narrow notches leading to very small cross section changes. Further, one must be careful to keep the notch fairly narrow, for otherwise the correspondence between the perturbed and unperturbed cross sections will be lost due to the gross nature of the perturbation, and the method will cease to provide a linear estimate of the sensitivity. On the other hand, when the notch is very small, an unwanted sensitivity is developed to the mesh of the radial integration employed in the numerical calculations.

Thus, in a practical situation it is necessary to have a definite procedure which avoids the problems mentioned above and which permits the comparison of the sensitivity in one situation with that in another. The procedure which has been developed is as follows:

(1) Since the semiclassical turning radius of the grazing partial wave is a point known to be of large sensitivity, we set $R^{\prime}=R_{t u r n}\left(l_{g}\right)$ and perform a series of calculations with a range of values for $a^{\prime}$. We chose a value of $a^{\prime}$ from these such that the $x^{2}$ of the perturbed calculation is 100 times larger than that of the unperturbed calculation for a given set of experimental data (or pseudo-data based on the unperturbed calculation).

(2) We then fix $a^{\prime}$ at this value and vary $R^{\prime}$ from some minimum to some maximum value, choosing a step size of about $0.2 \mathrm{fm}$. We choose the minimum, maximum, and step size so that this mesh is commensurate with the mesh of the radial integration of the calculation, $i . e$. , so that all values of $R^{\prime}$ used fall on integration mesh points.

(3) After the calculations over a range of $R^{\prime}$ values are performed, the value of $x^{2}$ of each calculation (as compared with some reference data set) is plotted as a function of the $R^{\prime}$ value used. This is the sensitivity function for the calculation.

The calculations were performed with a new version of the heavy ion optical model program HOP-TWO, version 4.1. This version of the code is modified to include the multiplicative notch function as one of the options for describing the potential, and to permit variation of $a^{\prime}$ or $R^{\prime}$ programmatically over a linear mesh of values. The program is described in Sec. 4.4 of this report.

16 Figures 12.3-2 through -4 show some sensitivity functions calculated for ${ }^{16} 0+{ }^{28}$ Si elastic scattering at several energies, using potential El8 as discussed in Sec. 12.1 of this report. Figure 12.3-2 shows the sensitivity function for a bombarding energy of $33 \mathrm{MeV}$, which is essentially at the Coulomb barrier for this reaction. Here the sensitivity function for the real potential forms a rather broad peak centered at about $9 \mathrm{fm}$ with a FWHM of about $2 \mathrm{fm}$, which provides excellent sensitivity to the nuclear potential in the tail region at this bombarding energy. The imaginary potential sensitivity function is double peaked, with a broad peak corresponding to that of the real potential in the tail region and a narrower peak with its maximum at $7.4 \mathrm{fm}$, which is actually inside the real potential radius of $7.5 \mathrm{fm} . R_{0}$ and $R_{c}$ in the figure are the nuclear and Coulomb radii. 


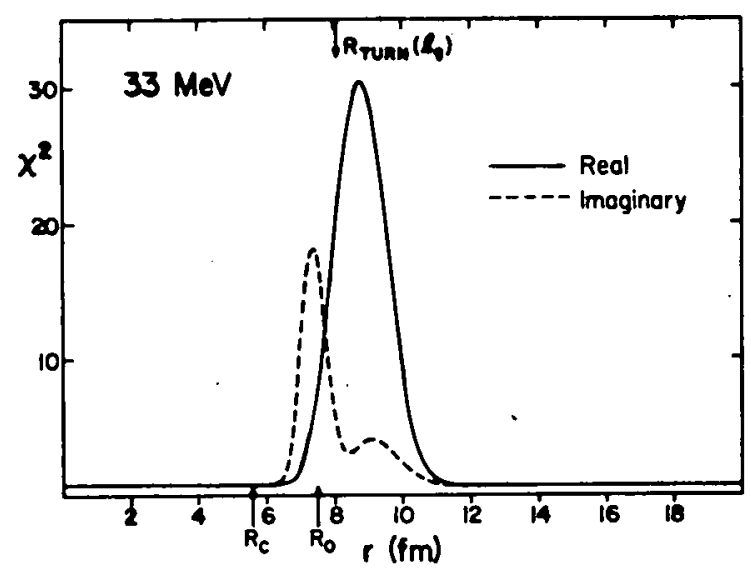

Fig. 12.3-2. Sensitivity function for potential El8 and $160+28 \mathrm{Si}$ at a bombarding energy of $33 \mathrm{MeV}$. For this calculation we have taken $a^{\prime}=0.431 \mathrm{fm}$.

Figure 12.3-3 shows the sensitivity function for the potential E18 at a bombarding energy of $53 \mathrm{MeV}$, which is in another important region. Here the sensitivity function is sharply peaked at $R_{\text {turn }}\left(l_{\dot{g}}\right)$ as indicated by the arrow in the figure. This condition of sharp localization of the real potential sensitivity at this point is found to be present in all data examined between 38 $\mathrm{MeV}$ and $81 \mathrm{MeV}$, and thus all data sets in this region measure the real potential at essentially the same point. We note, however, that the sensitivity function for the imaginary potential has three distinct peaks, none of which corresponds to the peak of the real potential. This provides evidence that. data in this region is actually sensitive to the imaginary potential over a larger radial region than that of the real potential. Thus, the imaginary potential is better determined in the surface region than is the real potential from data in this energy region.

Figure 12.3-4 shows the sensitivity function for a bombarding energy of $215.2 \mathrm{MeV}$. Here we see that the real potential function has two prominent peaks, the smaller at $R_{\text {turn }}\left(\ell_{g}\right)$ and the larger well inside this one and peaked at essentially the real potential radius of $7.5 \mathrm{fm}$. Again the imaginary potential is triple peaked and sensitive over a broader region than the real potential function. The inner peak in the real potential sensitivity function is probably
Fig. 12.3-3. Sensitivity function for potential El8 and $16_{0}+28 \mathrm{Si}$ at a bombarding energy of $53 \mathrm{MeV}$. For this calculation we have taken $a^{\prime}=0.057^{\prime} \mathrm{fm}$.
Fig. 12.3-4. Sensitivity function for potential El8 and $160+28 \mathrm{Si}$ at a bombarding energy of $215.2 \mathrm{MeV}$. For this calculation we have taken $a^{\prime}=0.012 \mathrm{fm}$.

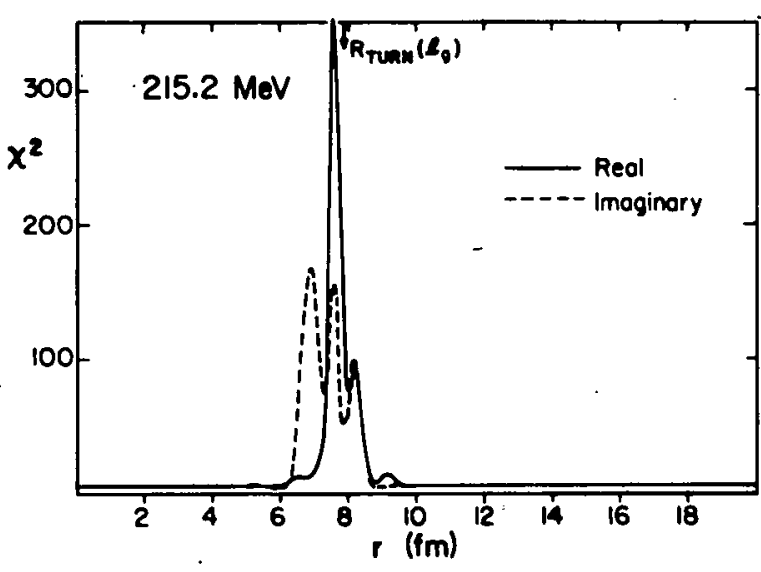


associated with the nuclear rainbow scattering effects which are prominent at these energies and lead to sensitivities deeper in the potential, particularly in the nuclear surface region.

Thus, a combination of data from the coulomb barrier region, the surface absorption region, and the nuclear rainbow region provides an effective means of mapping the nuclear potential over a relatively large range of radii. As discussed in Sec. 12.1, analys is of such a combination of data leads to a potential wh hich when characterized by an energy-independent Woods-Saxon form is quite shallow, about $10 \mathrm{MeV}$ in central depth.

It should be mentioned that another group at Brookhaven has develuped a technique somewhat related to this one which involves applying inner and outer radial cutoffs to the potential so as to determine the inner and outer limits of sensitivity. 1 They have applied their technique not only to elastic scattering but also to transfer reaction, and shown that elastic scattering is sensitive over a somewhat larger region than the transfer reactions studied. It is clear that the technique described here could also be used to investigate inelastic scattering and transfer. In particular, it would be interesting to see if there were certain reactions, e.g., transfers with poor angular momentum matching, which had enhanced potential sensitivities over a large radial region. However, these calculations even with elastic scattering are time consuming, and such an investigation of transfers might be prohibitively expensive in computer time.

An alternative approach which will be investigated soon is to apply the methods of perturbation theory to this type of perturbation. By this method it should in principle be possible to speed up the calculation of sensitivity functions, and could even lead to new ways of defining such functions.

1. P.J. Moffa, C.B. Dover, and J.F. Vary, Hhys. Kev. C 13, 14\% (19\%6).

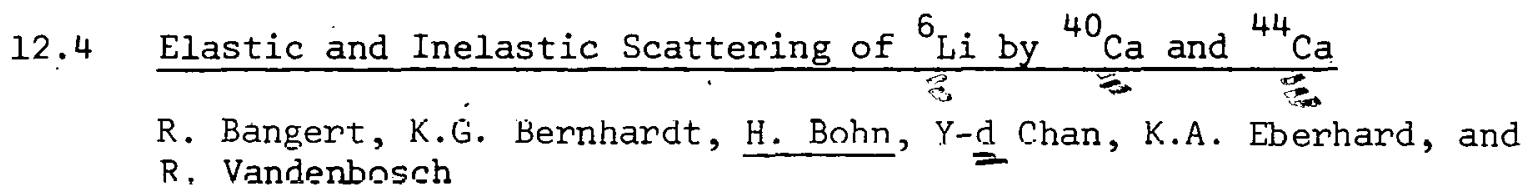

A large amount of angular distribution data exists for the elastic and inelastic scattering of ${ }^{4} \mathrm{He}$ and ${ }^{3} \mathrm{He}$ projectiles by $\mathrm{Ca}$ and various other isotopes over a large range of bombarding energies (20 MeV - $100 \mathrm{MeV}$ ). The experimental results were obtained in an attempt to gain a deeper understanding of the rause of the observed backward angle "anomalies" and their dependence on bombarding energy and target nucleus structure.

In contrast, only little equivalent experimental information is available for the scattering of $\mathrm{Li}$ projectiles, particularly by $\mathrm{Ca}$ isotopes where the isotopic dependence of the backward angle cross section is strong for ${ }^{4} \mathrm{He}$ scattering. Besides this and the fact that the mass of $\mathrm{Li}$ is between the one for He and heavier ions, the study of $\mathrm{Li}$ induced scattering and reactions could be interesting in itself because of the cluster structure of ${ }^{6} \mathrm{Li}$ and ${ }^{7} \mathrm{Li}$ nuclei. For example, this cluster structure seems to influence the cross section behavior 
for $\left({ }^{7} \mathrm{Li}, \alpha \times \mathrm{xn}\right)$ - and $\left({ }^{7} \mathrm{Li}, \mathrm{txn}\right)$-type reactions vs $\left({ }^{7} \mathrm{Li}, \mathrm{xn}\right)$ fusion reactions on heavy 2 and medium heavy ${ }^{3}$ target nuclei.

We have measured complete angular distributions $\left(30^{\circ} \leq \theta_{\mathrm{cm}} \leq 173^{\circ}\right)$ for the elastic and inelastic scattering of $30 \mathrm{MeV}{ }^{6} \mathrm{Li}$ by isotopically enriched ${ }^{40} \mathrm{CaF}_{2}$ and ${ }^{44} \mathrm{CaF}_{2}$ targets. The bombarding energy of $30 \mathrm{MeV}$ exceeds the Coulomb barrier by about the same energy as for the $25 \mathrm{MeV} 4 \mathrm{He}+\mathrm{Ca}$ case in which the strongest backward angle "anomalies" were observed. The $6_{\mathrm{Li}}$. events were detected in three standard $\mathrm{dE} / \mathrm{d} \mathbf{x}-E$ counter telescopes, separated by $\theta_{1 \mathrm{lab}}=15^{\circ}$. The use of the $\mathrm{dE} / \mathrm{dx}$ - E technique for measuring the cross sections for the elastic and inelastic ${ }^{6} \mathrm{Li}$ scattering was necessary due to the presence of a large background of projectile breakup particles (mainly ${ }^{4} \mathrm{He}$ ) over the whole angular range. In the PID spectrum the $6 \mathrm{Li}$ peak was well separated from the strong $4 \mathrm{He}$ peak. A window on the ${ }^{6} \mathrm{Li}$ peak allowed the accumulation of clean energy spectra for ${ }^{6} \mathrm{Li}$ scattering, from which the individual peak intensities were obtained.

The experimental results for the elastic scattering of ${ }^{6} \mathrm{Li}$ by ${ }^{40} \mathrm{Ca}$ and ${ }^{44} \mathrm{Ca}$ are compared in Fig. 12.4-1 together with optical model predictions. At forward angle the cross section fall-off is structureless and has the same slope for both ${ }^{40} \mathrm{Ca}$ and ${ }^{44} \mathrm{Ca}$. Above $\theta_{\mathrm{cm}}=70^{\circ}$ a diffraction structure is observed which seems to oscillate around the slope determined at forward angles and which is much more pronounced for ${ }^{40} \mathrm{Ca}$ than for ${ }^{44} \mathrm{Ca}$. At even larger angles the more unstructured fall-off seems to remain for ${ }^{44} \mathrm{Ca}$ (note the larger errors bars) whereas the diffraction structure for ${ }^{40} \mathrm{Ca}$ becomes more and more pronounced and finally oscillates
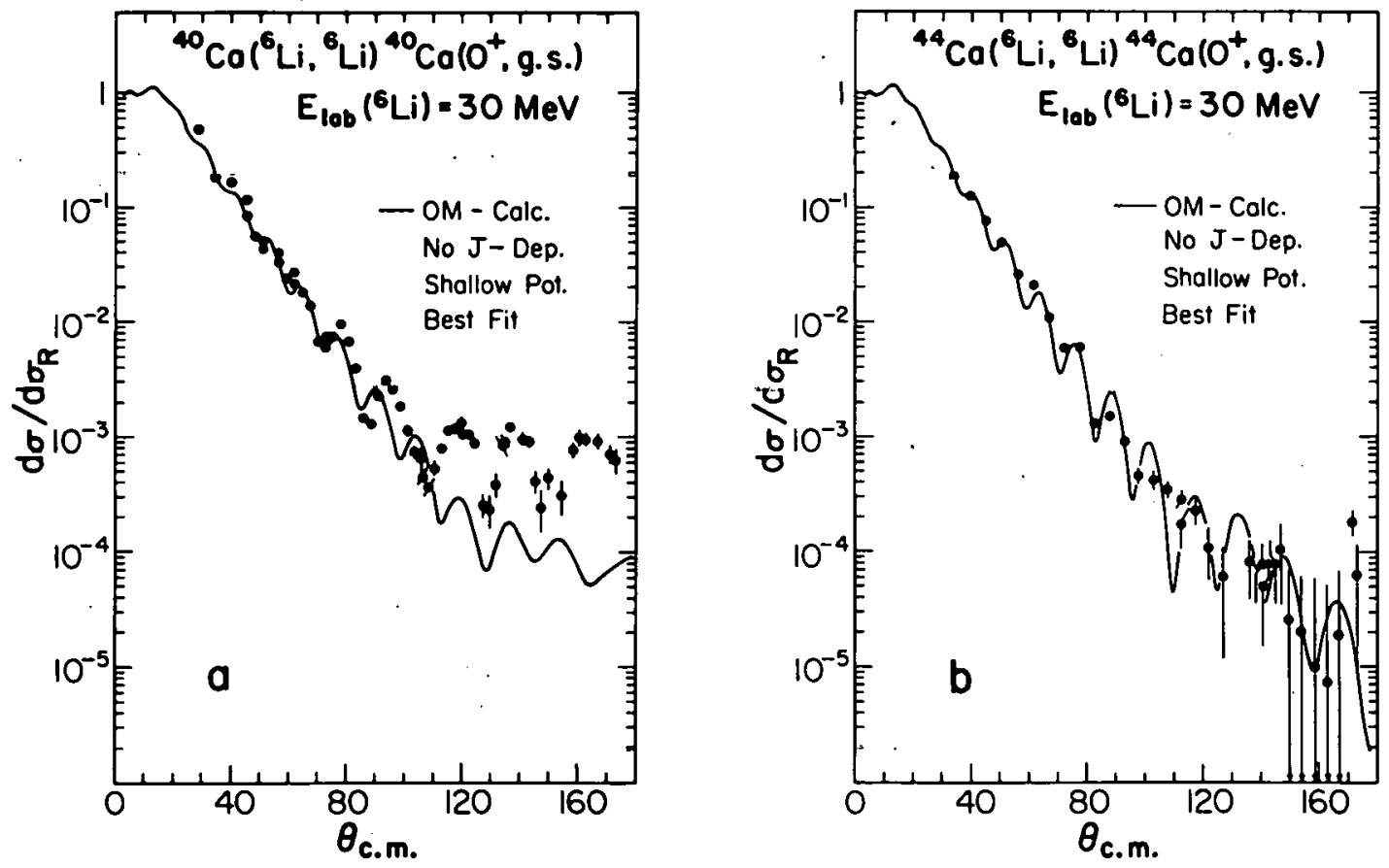

Fig. 12.4-1. Experimental angular distributions for the elastic scattering of ${ }^{6}$ Li by $40 \mathrm{Ca}$ (a) and ${ }^{44} \mathrm{Ca} \mathrm{(b)} \mathrm{at} 30 \mathrm{MeV}$ bombarding energy. The solid lines represent optical model "best" fits (see Table 12.4-1). 
Table 12.4-1. Optical model parameters for the "best" fits to the experimental elastic $6 \mathrm{Li}+{ }^{40} \mathrm{Ca}\left({ }^{44} \mathrm{Ca}\right)$ scattering data.

$\begin{array}{llccccccc}\text { Target } & \begin{array}{l}E_{\mathrm{Lab}}^{\left({ }^{6} \mathrm{Li}\right)} \\ (\mathrm{MeV})\end{array} & \begin{array}{c}\mathrm{V} \\ (\mathrm{MeV})\end{array} & \begin{array}{c}r_{\mathrm{v}} \\ (\mathrm{fm})\end{array} & \begin{array}{c}\mathrm{a}_{\mathrm{v}} \\ (\mathrm{fm})\end{array} & \begin{array}{c}\mathrm{w} \\ (\mathrm{MeV})\end{array} & \begin{array}{c}r_{w} \\ (\mathrm{fm})\end{array} & \begin{array}{c}\mathrm{a}_{\mathrm{w}} \\ (\mathrm{fm})\end{array} & \\ { }^{40} \mathrm{Ca} & 29.8 & 25.7 & 1.200 & .693 & 22.0 & 1.200 & .693 & \text { Reference } 1 \\ { }^{44} \mathrm{Ca} & 30 & 24.6 & 1.222 & .610 & 56.1 & 1.010 & .696 & \text { Best fit } \\ { }^{4} & 30 & 24.6 & 1.204 & .634 & 56.1 & .994 & .678 & \text { Best fit }\end{array}$

Interaction range: $\quad R=r_{v}\left(A_{T}^{l / 3}+A_{p}^{l / 3}\right)$

Coulomb radius: $\quad R_{c}=1.4\left(A_{T}^{1 / 3}+A_{p}^{1 / 3}\right)$

at backward angles around a constant average cross section which is more than an order of magnitude larger than for ${ }^{44} \mathrm{Ca}$. Also indicated in Fig. 12.4-1 are optical model calculations using six parameter volume absorption type WoodsSaxon potentials. These fits are "best: fits (minimum $x^{2}$ ) starting from four parameter potentials, 1 which are even less capable of reproducing the measured back angle cross section behavior for ${ }^{40} \mathrm{Ca}$. Table $12.4-1$ summarizes the "best" fit parameters.

It is interesting to note that the "best" fit potentials tend to conserve the real potential depth and increase the absorption by increasing $W$ instead of $r_{W}$. Deep potentials $(V \approx 250 \mathrm{MeV})$, which gave the best agreement with the experimental data for $30 \mathrm{MeV}{ }^{6} \mathrm{Li}$ scattering from ${ }^{12} \mathrm{C}$ and 160 , especially for the strong raise of the cross section at backward angles, 1 also failed in reproducing our ${ }^{40} \mathrm{Ca}$ data. So far, only inclusion of $\mathrm{J}$-dependent absorption leads to a better matching of the backward angle cross section magnitude and structure for the ${ }^{40} \mathrm{Ca}$ case, whereas the fit to the more forward angle part then deteriorates both in phase and magnitude.

The energy spectra could be analyzed for the $\left(3^{-}, 3,74 \mathrm{MeV}\right)$-state in ${ }^{40} \mathrm{Ca}$ and for the $\left(2^{+}, 1.16 \mathrm{MeV}\right)-$ state in ${ }^{44} \mathrm{Ca}$. Due to the target thickness the $3^{-}-$state cross section may contain contributions from the $\left(0^{+}, 3.35 \mathrm{MeV}\right)$ and $\left(2^{+}, 3.90 \mathrm{MeV}\right)$ states in ${ }^{40} \mathrm{Ca}$. The inelastic angular distributions are presented in Fig. 12.4-2 together with results of coupled channel calculations. The cross section behavior is similar to the one observed for the elastic scattering: The cross sectinn at forward angles decreases for both targets but whereas the fall-off for. the ${ }^{6} \mathrm{Li}+$ ${ }^{44} \mathrm{Ca}\left(2^{+}\right)$inelastic scattering continues, the ${ }^{6} \mathrm{Li}+{ }^{40} \mathrm{Ca}\left(3^{-}\right)$inelastic scattering cross sections remain constant at backward angles and are an order of magnitude larger.

The coupled channel calculations performed thus far have used the "best" fit optical model parameters mentioned above without J-dependent absorption and without complex coupling. The couplings we used were_( $0^{+}$, g.s.) $-\left(3^{-}, 3.74 \mathrm{MeV}\right)$, $B_{3}=0.25$ for ${ }^{40} \mathrm{Ca}$ and $\left(0^{+}, g . s.\right)-\left(2^{+}, 1.16 \mathrm{MeV}\right)-\left(3^{-}, 3.30 \mathrm{MeV}\right), B_{2}=0.25, B_{3}=$ 0.20 for ${ }^{44} \mathrm{Ca}$ and are the same as those used in Ref. 4 . For ${ }^{44} \mathrm{Ca}$ the coupling 

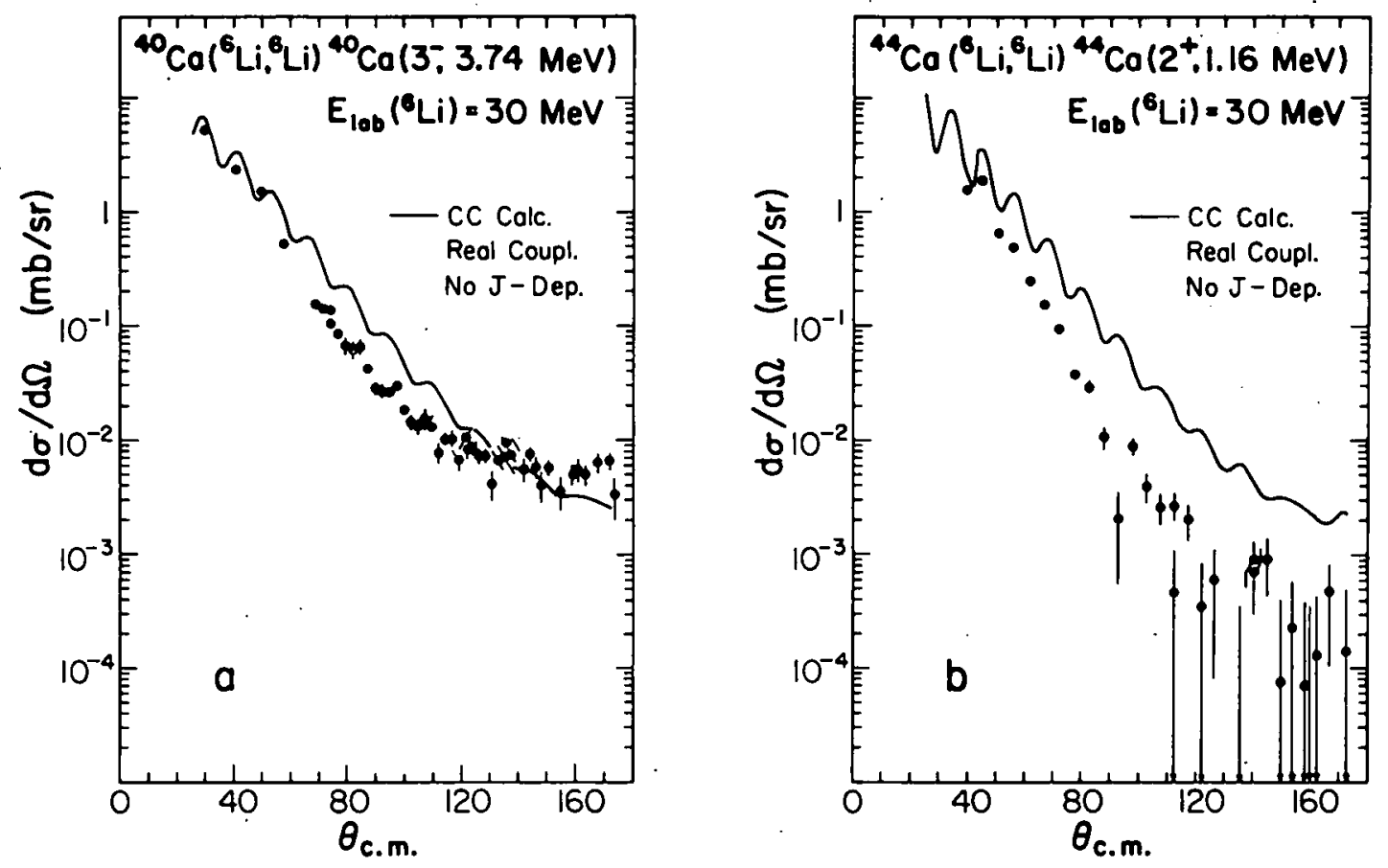

Fig. 12.4-2. Experimental angular distributions for the inelastic scattering of ${ }^{6} \mathrm{Li}$ to the $\left(3^{-}, 3.74 \mathrm{MeV}\right)$-state in $40 \mathrm{Ca}(\mathrm{a})$ and to the $\left(2^{+}, 1.16 \mathrm{MeV}\right)$-state in ${ }^{44} \mathrm{Ca} \mathrm{(b)} \mathrm{at} 30 \mathrm{MeV}$ bombarding energy. The solid lines represent results of coupled channel calculations.

to the $\left(3^{-}, 3.30 \mathrm{MeV}\right)$-state seems to be reasonable, because we were able to resolve the corresponding peak in the energy spectra at least at the most forward angles. It should be noted that the imaginary potential depths used in the coupled channel calculations are not reduced with respect to the optical model calculations. The following results were obtained from the coupled channel calculations: The elastic ${ }^{6} \mathrm{Li}+{ }^{40} \mathrm{Ca}$ scattering is described only in an averaged way, this means the experimentally observed pronounced back angle structure is not reproduced. Inclusion of complex coupling for this special case reduces only the magnitude of the fit at. backward angles by a factor of about two without improving the fit to the diffraction structure. In contrast to that for the elastic ${ }^{6} \mathrm{Li}+{ }^{44} \mathrm{Ca}$ scattering not even an overall agreement of the fit with the data is achieved, e.g., the fit lies far above the data points and the agreement is not improved even if one would omit the coupling to the $\left(3^{-}, 3.30\right)$-state in ${ }^{44} \mathrm{Ca}$. The situation is similar for the inelastic scattering as indicated in Fig. 12.4-2. It would be interesting to see how the inclusion of complex coupling and $\mathrm{J}$-dependent absorption would influence the quality of the fit to the data.

Possible contributions to the experimental ${ }^{6} \mathrm{Li}+{ }^{40} \mathrm{Ca}\left({ }^{44} \mathrm{Ca}\right)$ elastic scattering cross sections due to compound nucleus formation were estimated by HauserFeshbach calculations (STAT2). Using reasonable potential parameters from the literature for the $\mathrm{n}, \mathrm{p}, \mathrm{d},{ }^{3} \mathrm{He}$ and ${ }^{4} \mathrm{He}$ exit channels and our optical model best fit parameters (see Table $12.4-1$ ) for the ${ }^{6} \mathrm{Li}+{ }^{40} \mathrm{Ca}\left({ }^{44} \mathrm{Ca}\right)$ channel, we obtain 
for the total $\mathrm{H}-\mathrm{F}$ cross section to the $\left(0^{+}, \mathrm{g} . \mathrm{s}.\right)$-state in ${ }^{40} \mathrm{Ca}\left({ }^{44} \mathrm{Ca}\right) 1 \times 10^{-5} \mathrm{mb}$ $\left(6 \times 10^{-7} \mathrm{mb}\right)$. This is already more than two orders of magnitude lower than the corresponding averaged experimental differential cross sections at backward angles.

$$
\text { In case of }{ }^{40} \mathrm{Ca} \text {, a sizable }
$$

$\left({ }^{6} \mathrm{Li},{ }^{4} \mathrm{He}\right)$ yield could also be identified Figure 12.4-3 shows the experimental angular distribution for the formation of ${ }^{42} \mathrm{Sc}$ in its $0^{+}$ground state. The cross sections are comparable in size to those for the elastic scattering of $\sigma_{\mathrm{Li}}$ by $40 \mathrm{Ca}$. Because the corresponding total $\mathrm{H}-\mathrm{F}$ cross section for the formation of $42 \mathrm{Sc}\left(\mathrm{O}^{+}, \mathrm{g.s}\right.$.) is only about $1 \times 10^{-6} \mathrm{mb}$, the ${ }^{40} \mathrm{Ca}\left({ }^{6} \mathrm{Li},{ }^{4} \mathrm{He}\right)^{42} \mathrm{Sc}\left(0^{+}, \mathrm{g} . \mathrm{s}.\right)$ reaction seems to be mainly direct. A similar conclusion is extracted from the forward angle part of angular distributions obtained for the $\left({ }^{6} \mathrm{Li},{ }^{4} \mathrm{He}\right)$ reaction on lighter nuclei. 5 Not very much is known about the reaction mechanism of $\left(6 \mathrm{Li},{ }^{4} \mathrm{He}\right)$ reactions on calcium nuclei. It would therefore be interesting to extend the present information obtained in heavier mass regions 2 to the $A=40 \mathrm{re}-$ gion.

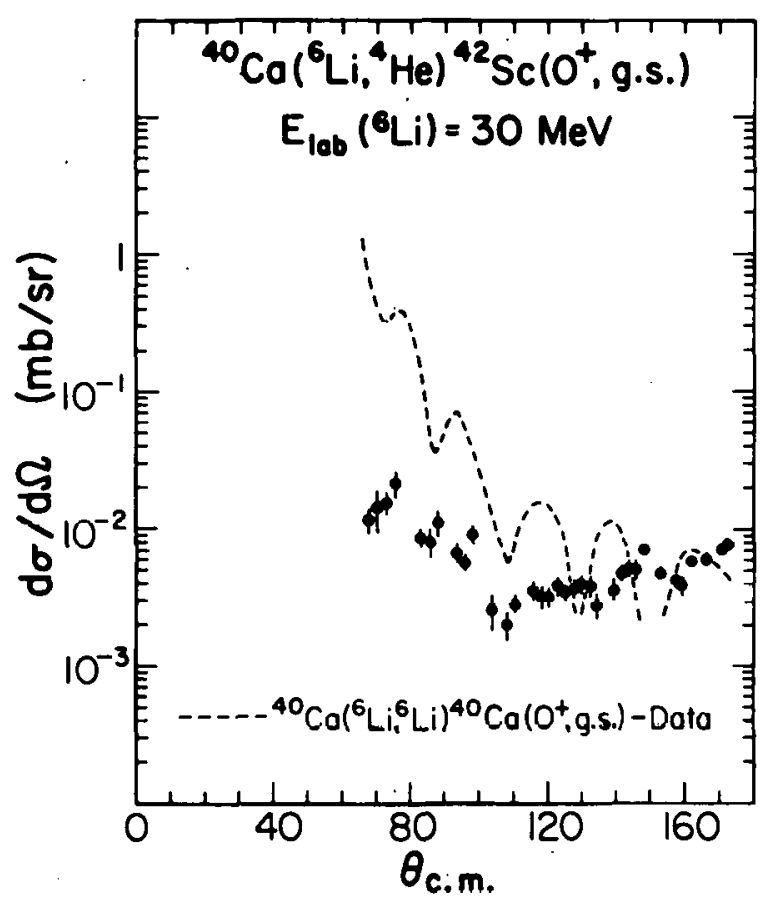

Fig. 12.4-3. Experimental angular distribution for the reaction ${ }^{40} \mathrm{Ca}\left({ }^{6} \mathrm{Li},{ }^{4} \mathrm{He}\right)$ $42 \mathrm{Sc}\left(0^{+}, \mathrm{g} . \mathrm{s}.\right)$ at $30 \mathrm{MeV}$ bombarding energy. The dashed line represents the differ-. ential cross sections for the elastic $6 \mathrm{Li}+{ }^{40} \mathrm{Ca}$ scattering.

1. V.V. Davydov, B.G. Novatskii, A.A. Ogloblin, S.B. Sakuta, D.N. Stepanov, and V.I. Chuev, IZV. Akad. Nauk SSSR, Ser. Fiz. 35, 2399 (1971);

V.I. Chuev, V.V. Daviolov, B.G. Novatskii, A.A. Ogloblin, S.B. Sakuta, and D.N. Stepanov, Journal de Physique C 6, 157 (1971).

2. H. Frelesleben, H.C. Britt, J. Birkelund, and J.R. Huizenga, Phys. Rev. C.10, $245(1974)$.

3. H. Bohn, J.G. Cramer, G. Daniel, P. Kienle, M.R. Maier, and D. Proetel, Annual Report, Munich (1371), p. 27.

4. W. Trombik, K.A. Eberhard, and J.S. Eck, Phys. Rev. C 11, 685 (1975).

5. See for example: J.D. Garrett, R. Middleton, D..J. Pullen, S.A. Andersen, 0. Nathan, and 0 . Hansen, Nucl. Phye, A164, 449 (1271). 
12.5 Elastic Scattering of ${ }^{14} \mathrm{~N}$ by ${ }^{12} \mathrm{C}$ from 33.0 to $48.0 \mathrm{MeV}$

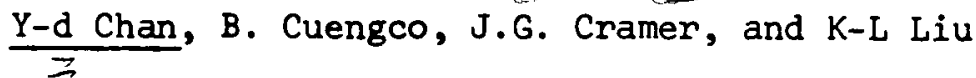

In addition to the angular distribution data for the elastic scattering of ${ }^{14} \mathrm{~N}$ from ${ }^{12} \mathrm{C}$ reported previously, 1 excitation function at four different angles $\left(\theta_{\mathrm{cm}}=43.5^{\circ}, 54.5^{\circ}, 130^{\circ}\right.$ and $\left.140^{\circ}\right)$ have been measured in the energy range of $E_{\mathrm{cm}}=12.0-26.0 \mathrm{MeV}$ (Fig. 12.5-1). It is hoped that these measurements would help us to determine a better set of optical potential parameters. The magnitude of the cross sections at very backward angles was found to be quite sensitive to the parameters. 1

The previous report of this experiment emphasized the interpretation of the elastic transfer mechanism ${ }^{2}$ within the DWBA picture, and numerical calculations were reported. I In the present report, we will summarize some other attempts we have made to fit the data.

(1) Excitation function. As a starting point, best fit optical parameters obtained from $E_{1 a b}=33.0,38.0$, 41.0 , and $44.0 \mathrm{MeV}$ angular distribution data were used to deduce a linear energy dependence in $\mathrm{W}$, the imaginary potential strength, and this potential was then treated as an initial guess for a search to obtain a best fit to the excitation function. This scheme was not very successful; reasonable fits were obtained only within a certain narrow energy range. An example of one of such trials is indicated by the dashed curve in Fig. 12.5-1. It does not fit backward angle excitation functions at all. This is not surprising, since there are fluctuations and the elastic transfer mechanism may dominate this region. It therefore is very unlikely that the cross section can be accounted for by a simple optical potential. It is planned to try a more complex energy dependence in both the real and imaginary potentials in order to improve the fit.

\section{(2) Compound elastic contribu-} tions. In order to justify the picture of a direct elastic transfer mechanism, compound nuclear contributions must be checked. A Hauser-Feshbach computer code STAT ${ }^{3}$ was applied to estimate the compound elastic contribution; the results are plotted in Fig. 12.5-2. Besides the $14 \mathrm{~N}+{ }^{12} \mathrm{C}$ channel, four others,

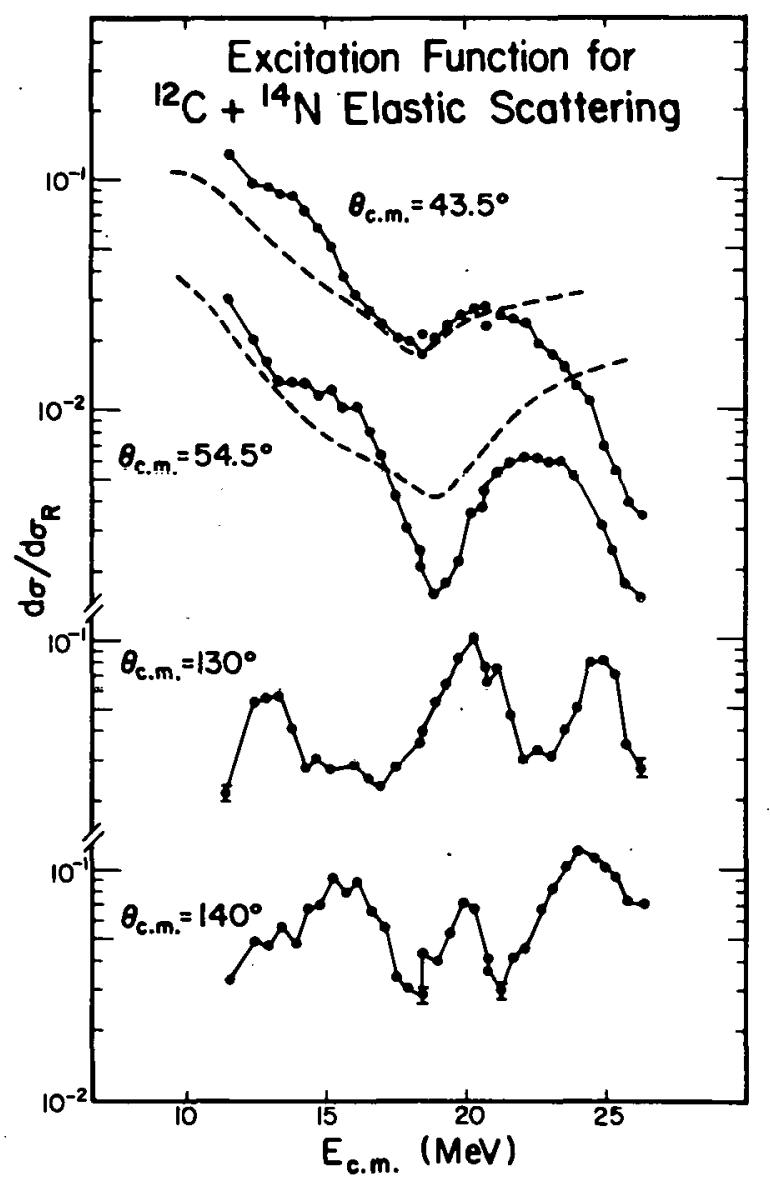

Fig. 12.5-1. Excitation functions for ${ }^{14} \mathrm{~N}+{ }^{12} \mathrm{C}$ elastic scattering. 
including $\mathrm{n}+{ }^{25} \mathrm{Al}, \mathrm{d}+{ }^{24} \mathrm{Mg}, \mathrm{p}+{ }^{25} \mathrm{Mg}$ and ${ }^{6} \mathrm{Li}+{ }^{20} \mathrm{Ne}$ were coupled simultaneously for each calculation. As seen from the figure, the compound elastic contributions are small except in some valley regions. However, due to the uncertainty of parameters we used in doing the calculations, this estimate should be considered only as a rough indication and not as a basis for neglecting the $\mathrm{CN}$ contributions.

(3) Two-state approximation 4 calculations. By considering the ${ }^{14} \mathrm{~N}$ nucleus as a ${ }^{12} \mathrm{C}$ core plus two nucleone, one can formulate the calculation so that the major contribution is mainly due to the exchange of the cores. Such a derivation is given, for example, in Ref. 4. To first order, this method and the DWBA calculations should yield similar results. The computer code TRANSIT $^{5}$ was used to calculate the cross sections: Results are shown by solid curves in Fig. 12.5-2. The quality of the fits is comparable to that with DWBA calculations. Optical model parameters in both cases are identical for each energy.

It is concluded from the data fitting that for the 12 to $26 \mathrm{MeV}$ energy range there may be several equally strong contributions. A simple elastic transfer cannot explain the backward data as satisfactorily as in the lower energy case..$^{4}$ However, the data do indicate that the backward rising phenomenon is still very pronounced in this region, even though a simple quantitative description is difficult to obtain.

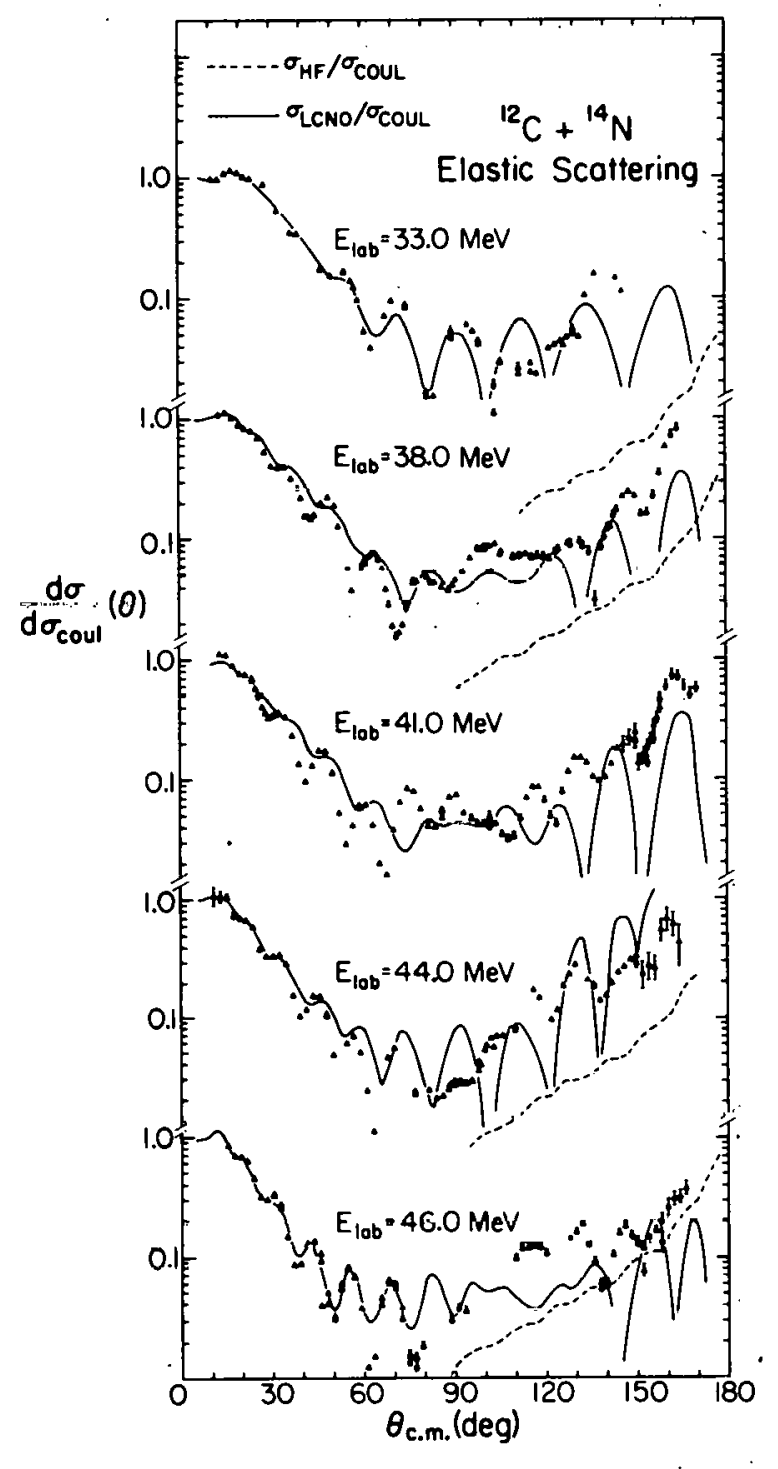

Fig. 12.5-2. HF calculations (dashed line) and LCNO calculations (solid line) for the elastic scattering of $14 \mathrm{~N}$ from 12C. Optical model parameters used for the LCNO calculations are identical to those shown in Ref. 1.

\footnotetext{
1. Nuclear Physics Laboratory Annual Report, University of Washington (1975), p. 112.

2. C.A. McMahon and W. Tobocman, Nucl. Phys. A202, 561 (1973).

3. STTAT2 by R. Stokstad, Oak Ridge National Laboratory, unpublished.

4. W. von Oertzen and H.G. Bohlen, Phys. Report 19C, No. I, June 1975.

5. Program TRANSIT, kindly communicated to us by H.G. Bohlen and W.von Oertzen.
} 
12.6 The Elastic Scattering of ${ }^{16} \mathrm{O}$ from ${ }^{14} \mathrm{C}$

K.G. Bernhardt, K.A. Eberhard, R. Vandenbosch, and M. Webb

The elastic scattering of ${ }^{16} \mathrm{O}$ from ${ }^{14} \mathrm{C}$ is of particular interest for two reasons. In the first place, both ${ }^{14} \mathrm{C}$ and 160 have relatively high-lying first excited states at about $6 \mathrm{MeV}$ excitation energy. Even though the Q-values for transfer channels are somewhat more favorable for carrying away the angular momentum brought in by grazing coliisions for the $160+1{ }^{4} \mathrm{C}$ case, the similarity in the inelastic channels may make the behavior of the elastic scattering in this system comparable to that of $160+160$, where inelastic scattering is known to play an important role. 1

The second reason for interest in this system is that ${ }^{16} 0+{ }^{14} \mathrm{C}$ leads, for the same center of mass energy, to only a slightly (.9 MeV) smaller excitation energy in the same compound nucleus as $180+12 \mathrm{C}$. In the latter system some correlated intermediate structure dominated by odd partial waves has been found in the elastic and $\alpha$-channels. ${ }^{2}$ One of the explanations put forward for this effect suggested that the odd spin yrast levels in the vicinity of the grazing partial wave may be displaced up in energy relative to the even yrast levels. This reduced availability of odd spin levels in contrast to the even spin levels could lead to a stronger concentration of odd spin strength in energy. If this explanation were true one could look for similar effects in the $160+14 \mathrm{C}$ system at the appropriate energies.

Using a kinematic coincidence technique described previously, ${ }^{3}$ we have measured excitation functions over the energy range 15 to $30 \mathrm{MeV}$ (c.m.) at angles between $\theta_{\mathrm{c} . \mathrm{m} .}=65^{\circ}$ and $110^{\circ}$. The data are displayed in Fig. 12.6-1. Gross structure about 1-2 MeV (c.m.) broad and shifting with angle is observed, reminisrent of the behavior exhibited by the $160+160$ system. The average cross sections are about a factor of 20-50 bigger than for the elastic scattering of 180 from ${ }^{12} \mathrm{C}$, where a Hauser-Feshbach calculation established fairly large compoundelastic contributions. This suggests a more direct reaction mechanism for the $160+{ }^{14} \mathrm{C}$ case. A preliminary correlation analysis of 8 excitation functions showed no prominent deviation at the appropriate excitation energies in ${ }^{30} \mathrm{Si}$ corresponding to energies where odd spin correlated structure is exhibited by the $18_{0}+12 \mathrm{C}$ elastic scattering. The strongest anomaly found in the $16_{\mathrm{O}}+14 \mathrm{C}$ correlation analysis is around $E_{c . m .}=23.5 \mathrm{MeV}$. This anomaly shows up as well in some of the simultaneously measured ${ }^{14} \mathrm{C}\left(16_{0},{ }^{18_{0}}\right)^{12} \mathrm{C}$ reaction data.

In addition to the excitation functions, we have measured but not yet completely analyzed angular distributions at $E_{c . m}=21.9$ and $23.5 \mathrm{MeV}$ for the angular range from $\theta_{\mathrm{c} . \mathrm{m} .}=40^{\circ}$ to $120^{\circ}$. The raw data show fairly pronounced structure with the average cross section increasing at backward angles. The periodicity, however, cannot be reproduced by a single partial wave.

In summary, our present results indicate a structure which is similar to though not as dramatically enhanced as the $16_{0}+16_{0}$ case. No prominent odd spin structure is observed, in contrast to the $180+12 \mathrm{C}$ system. If the contribution to these structures were of the same order of magnitude as for the $18_{0}+12 \mathrm{C}$ case a large direct component in the $160+14 \mathrm{C}$ could prevent us from seeing a 


\section{${ }^{14} \mathrm{C}+{ }^{16} \mathrm{O}$ Elastic Scattering}

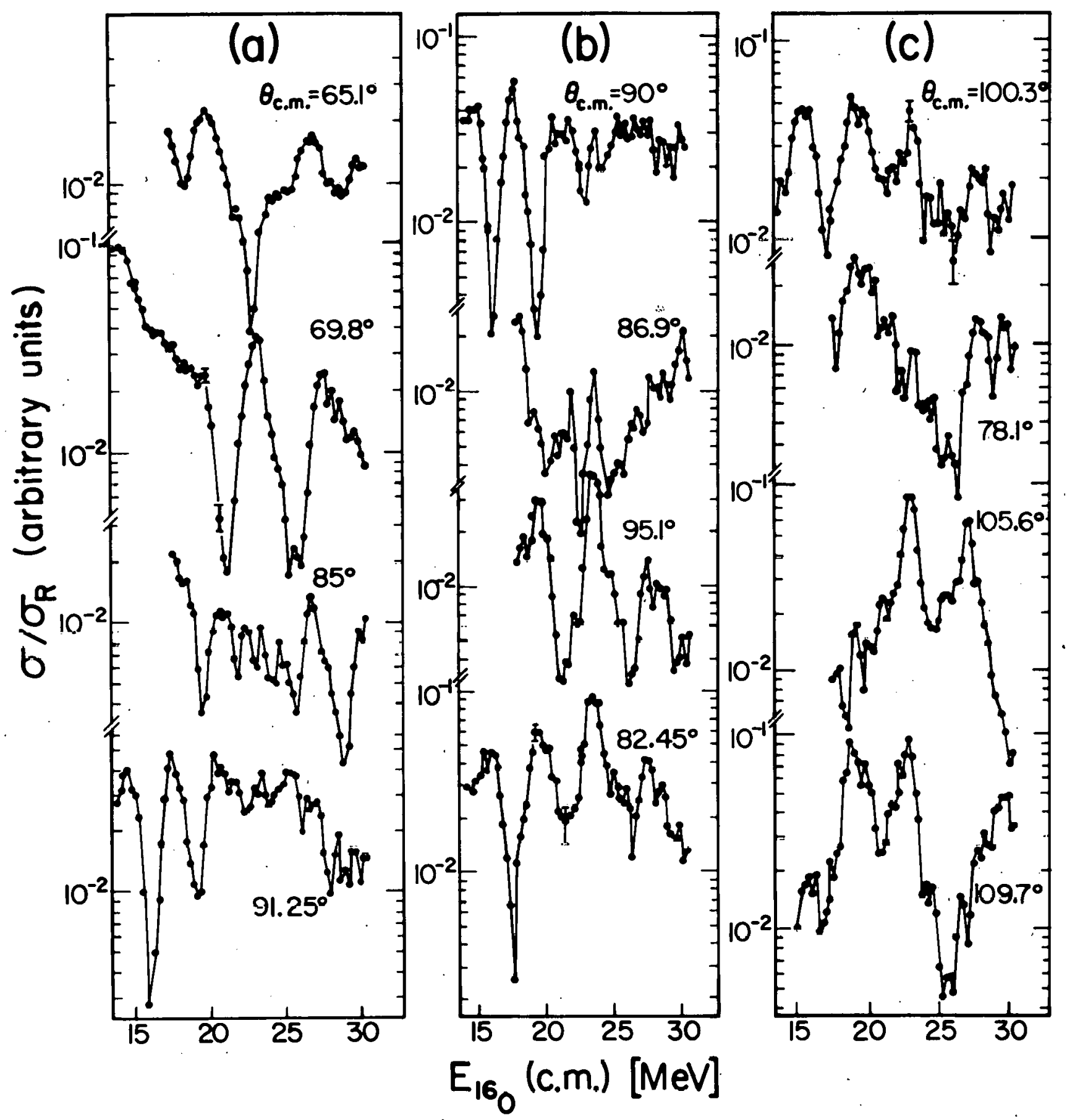

Fig. 12.6-1. ${ }^{16} \dot{0}+{ }^{14} \mathrm{C}$ elastic scattering excitation functions. 
correlation. An optical model analysis of the ${ }^{16} \mathrm{O}+{ }^{14} \mathrm{C}$ elastic scattering will enable us to estimate the compound elastic contribution with a Hauser-Feshbach calculation and show in a more quantitative way whether in principle we could observe the same kind of structure as in the $180+12 \mathrm{C}$ system.

1. J.V. Maher, M.W. Sachs, R.H. Siemssen, A. Weidinger, and D.A. Bromley, Phys. Rev. 188, 1665 (1969).

2. M.P. Webb, B. Vandenbosch, K.A. Eberhard, K.G. Bernhardt, and M.S. Zisman, Phys. Rev. Lett. 36, 779 (1976).

3. Nuclear Physics Laboratory Annual Report, University of Washington (1975), p. 121 .

12.7 Elastic Scattering of ${ }^{28} \mathrm{Si}$ from ${ }^{26} \mathrm{Mg}$

R. Bangert, Y-d Chan, J.G. Cramer, K-L Liu, C.K. Gelbke, and J. Wiborg

The ability to do particle identification around $A=28$ region with the gas counter telescopel was used to study the ${ }^{28} \mathrm{Si}+26 \mathrm{Mg}$ reaction at $80 \mathrm{MeV}$. Since we expected a large cross section from the proton transfer, forming ${ }^{27} \mathrm{Al}$, the PID spectrum would include $\mathrm{Si}, \mathrm{Al}$ and $\mathrm{Mg}$ in the PID range. The alpha-transfer cross section was also anticipated to be large, thus the $24 \mathrm{Mg}$ coming from this and the ${ }^{26} \mathrm{Mg}$ coming from elastic recoil could be compared to check the mass separation. The results of this study are shown in Fig. 12.7-1.

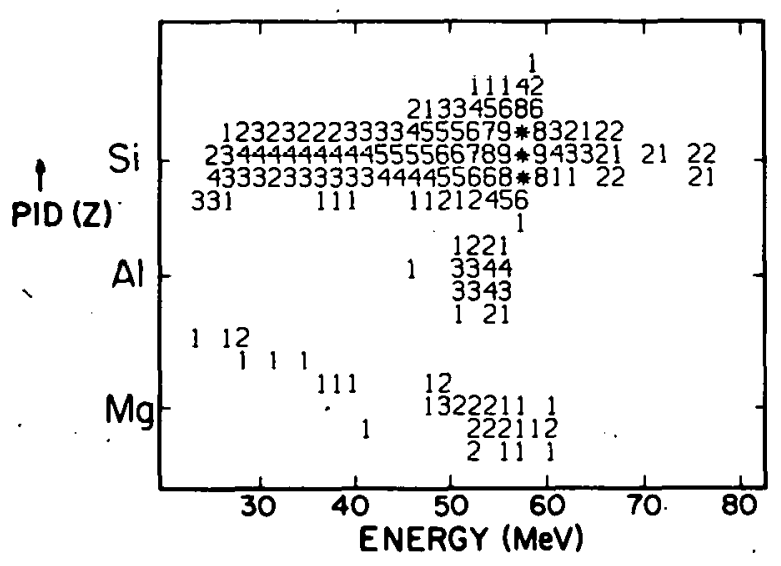

Fig. 12.7-1. Two-dimensional plot of particle energy vs PID. The reaction is ${ }^{28} \mathrm{Si}+26 \mathrm{Mg}$ at $80 \mathrm{MeV}$.

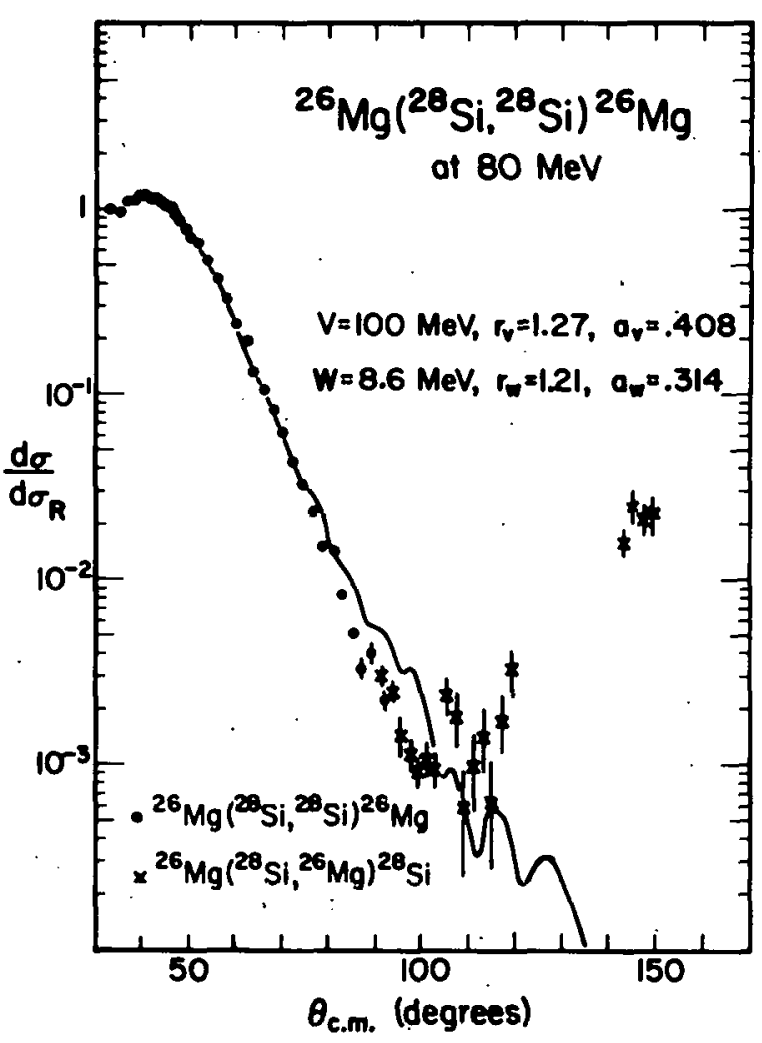

Fig. 12.7-2. Angular distribution of $26 \mathrm{Mg}\left(28_{\mathrm{Si}},{ }^{28}{ }_{\mathrm{Si}}\right) 26 \mathrm{Mg}$ at $80 \mathrm{MeV}$. 
These results indicate that charge separation is quite good, but isotope separation for the same $Z$ has not been achieved. (This is probably due: to the fact that the length of flight in the gas ionization region varies depending on the direction of flight.)

The ${ }^{26} \mathrm{Mg}\left({ }^{28} \mathrm{Si},{ }^{28} \mathrm{Si}\right){ }^{26} \mathrm{Mg}$ elastic cross section is shown in Fig. 12.7-2, together with an optical potential fit. There is strong indication of elastic

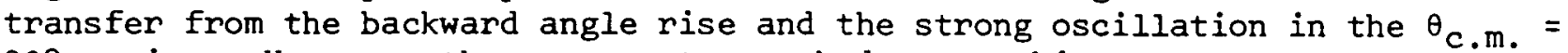
$90^{\circ}$ region. However, there are not enough data at this moment to warrant comparison with DWBA callcul.ations.

1. See Sec. 3.3 of lils lepurl.

12.8 Effects of Non-local Potentials in Heavy Ion Reactions

J.G. Cramer, R.M. DeVries ${ }^{\dagger}$, and W.G. Lynch

In last year's report we discussed the application of non-local potentials to heavy ion scattering and reactions and demonstrated that the approximations developed for non-local effects in light-ion reactions work very well in heavy ion reactions despite the shorter wave lengths.l We wish here to present recent work on the application of the local energy approximation (LEA) to finite range DWBA calculations so that the effects of nonlocality on transfer reactions can be studied. We will further discuss some extensions of the ideas of nonlocality which may be important for heavy ion reactions.

\section{DWBA Calculations with Non-Local Potentials}

We have applied the local energy approximation (LEA) to the case of a single-nucleon heavy ion transfer reaction using a modified version of the finiterange DWBA program LOLA. 2 We have used for this study the reaction $42 \mathrm{Ca}(160,15 \mathrm{~N}){ }^{43} \mathrm{Sc} *\left(1.18 \mathrm{MeV}, 3 / 2^{-}\right)$, a case which was not well fitted by analysis using DWBA with local potentials. ${ }^{3}$ We have used the optical model parameters of Ref. 3. Figure 12.8-1 shows the results of these calculations, and gives angular distributions corresponding to a range of values for the Gaussian non-locality parameter $\beta$ : $\beta=0,0.4$, and $1.0 \mathrm{fm}$. The latter value of $\beta$ is outside the range of validity of the LEA and is shown only as an extremum. We see from this example that for nonlocal ranges of $0.4 \mathrm{fm}$ or less the angular distribution is negligibly changed by the Perey damping of the wave functions. 4

We can understand this result in terms of the amount of absorption in the optical potentials used. Light ion potentials are often rather weakly absorbing in the interior allowing the Perey effect full sway. For this heavy ion reaction, however, the interior contributions to the DWBA cross section are strongly damped by the use of a strongly absorbing ( $W / V=40 / 100)$ optical potential. This assertion can be verified by reducing the absorption to $\mathrm{V} / \mathrm{W}$. $=5 / 100$ as shown in Fig. 12.8-2. Now, a $B=0.4 \mathrm{fm}$ is capable of strongly changing the predicted 


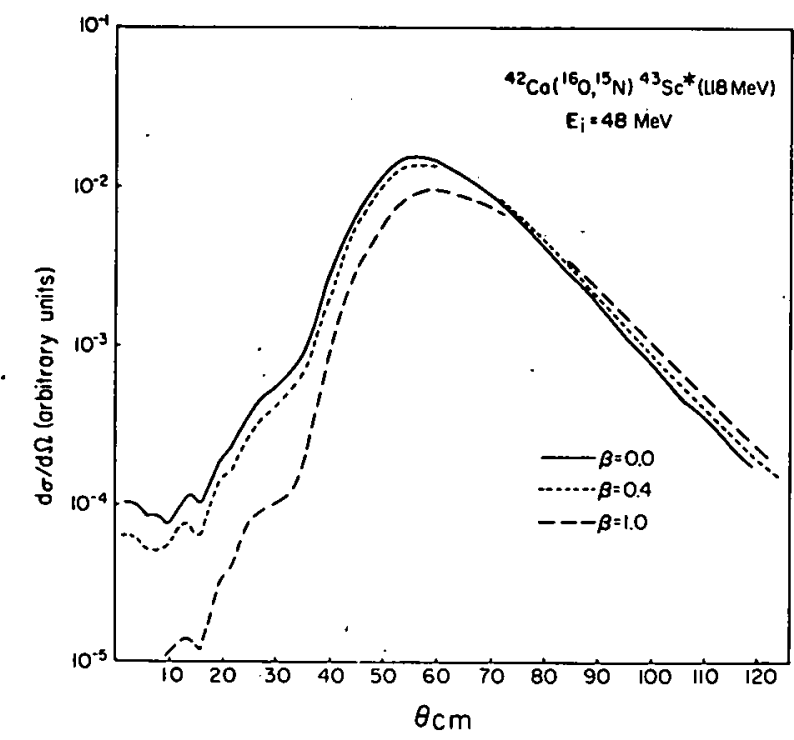

Fig. 12.8-1. Effect of Perey damping on DWBA calculation of $42(16 \mathrm{O}, 15 \mathrm{~N}) 43 \mathrm{Sc} *$ at $E=48 \mathrm{MeV}$, using a strongly absorbing potential. Potential used is given in Ref. $7(\mathrm{~W} / \mathrm{V}=40 / 100)$ and wave functions in the entrance and exit channels were damped using Eq. 2.

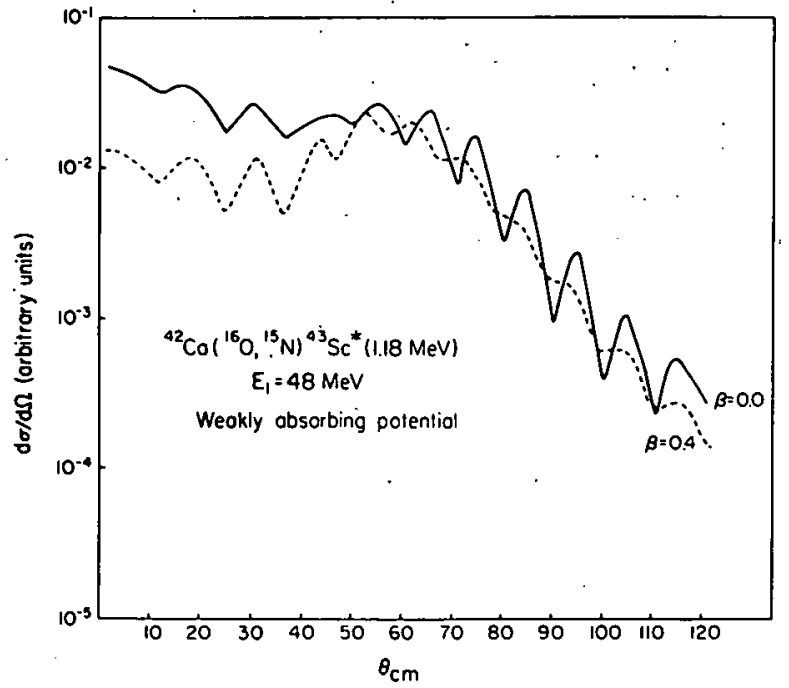

Fig. 12.8-2. Effect of Perey damping on a DWBA calculation for ${ }^{42} \mathrm{Ca}(16 \mathrm{O}, 15 \mathrm{~N}){ }^{4} 3 \mathrm{Sc} *$ at $E=48 \mathrm{MeV}$, using a weakly absorbing potential. Potential used is that given in Ref. 7, except that $W / V$ is taken as $5 / 100$.

angular distribution. This if heavy ion reactions are found in which weakly absorbing potentials are appropriate, nonlocality effects might be of concem.

At this point there remains an uncertainty about that non-local range is appropriate for heavy ions. We have only the theoretical guidelines of Jackson and Johnson. 5 They derive a simple model which yields a non-local range of approximately $0.2 \mathrm{fm}$ for alphas, fitting the observed energy dependence of alpha elastic scattering potentials rather well. With this model they predict heavy ion values to be given by

$$
\beta \simeq \beta_{\text {nucleon }} / A_{\text {ion }} \quad \text { or } \quad B \simeq 0.05 \text { fm for }{ }^{16} 0 \text {. }
$$

These small $\beta$ parameters, of course, suggest that the energy dependence of heavy ion optical potentials should be rather small. There is some evidence for this conclusion based on a recent analysis of $160+28 \mathrm{Si}$ elastic scattering between 33 and $215 \mathrm{MeV}, 6$ where a shallow energy-independent optical potential is found to give good fite to the data over this large energy range.

We conclude, then, that the effects of spherical Gaussian non-local potentials are quite small in heavy ion transfer DWBA calculations using strongly absorbing optical potentials. 
The failure of the spherical Guassian form of non-locality, as suggested by Perey and Buck, 4 to account for some of the discrepancies between heavy ion DWBA calculations and experimental data has prompted us to examine other possible forms of non-locality as possible explanations of these discrepancies. Such forms must satisfy the requirements that they are symmetric in the variables $\vec{r}$ and $\vec{r}^{\prime}$, and revert to a conventional local potential when the non-local range is made very small. In what follows we will ignore the role of the strictly local Coulomb potential. In general it can be inserted by substituting $E_{c m}-V_{C o u l}$ for $E_{c m}$.

\section{(1) Anti-commutator Form}

We suggest using a non-local potential of the general form:

$$
\overline{\mathrm{U}}=\left\{\mathrm{U}_{\mathrm{N}}, \mathrm{h}\right\}_{+}
$$

where $\bar{U}$ is the full non-local potential, $U_{N}$ is the potential form fartor (usually a Woods-Saxon or similar form), $h$ is the non-locality kernel, and $\{,\}_{t}$ is an anticommutation operation. This format for a nonlocal potential has the important characteristics that $\bar{U}$ is Hermetian if $U_{N}$ and $h$ are Hermetian, and the potential form factor $U_{N}$ now contributes only a physically appropriate values of $r$ and $r^{\prime}$. Further, the effective local potential $U_{L}$ can be easily obtained using a simple prescription:

$$
\mathrm{U}_{\mathrm{N}}=\frac{1}{\mathrm{f}(\mathrm{k})} \mathrm{U}_{\mathrm{L}}
$$

where $f(k)$ is the Fourier transform of the kernel function $h$ written in coordinate space with $\hbar^{2} k^{2} / 2 m=E_{C M}-U_{L}(r)$, i.e., $k(r)$ is the local momentum wave number. This makes the computation of effective local potentials very simple if we choose. kernel functions with well-known Fourier transforms.

For a Gaussian kernel of the type used by Perey and Buck, 4 i.e.,

$$
h\left(\left|\vec{r}-\vec{r}^{\prime}\right|\right)=\left[\begin{array}{ll}
\sqrt{\pi} & B
\end{array}\right]^{-3} \exp \left[-\left|\left(\vec{r}-\vec{r}^{\prime}\right) / \beta\right|^{2}\right]
$$

we obtain

$$
\mathrm{U}_{\mathrm{N}}=\exp \left[\beta^{2} \mathrm{k}^{2} / 4\right] \mathrm{U}_{\mathrm{L}}
$$

which agrees with the Perey-Buck non-local-to-local potential transformation. 7 If, on the other hand, we introduce a kernel of the Yukawa form:

$$
h\left(\left|\vec{r}-\vec{r}^{\prime}\right|\right)=\left(4 \pi \beta^{2}\left|\vec{r}-\vec{r}^{\prime}\right|\right)^{-1} \exp \left[-\left|\left(\vec{r}-\vec{r}^{\prime}\right) / \beta\right|\right]
$$

then the effective local potential is given by:

$$
U_{N}=\left(1+\beta^{2} k^{2}\right) U_{L}
$$

which amounts to an inverse linear energy dependence for the local potential. We note also that this is essentially the first two terms of a Taylor expansion of the Gaussian energy dependence of Eq. (5). 
Applying the anti-commutator form of a non-local potential to the timeindependent Schrödinger equation frequently results in significant simplifications:

$$
\left.\left[\mathrm{H}_{\mathrm{O}}+\mathrm{V}_{\mathrm{O}}+\frac{1}{2}\left[U_{\mathrm{N}}, \mathrm{h}\right\}_{+}-\mathrm{E}_{\mathrm{CM}}\right]|\psi\rangle\right\rangle=0
$$

with

$$
\hat{\psi}(\vec{r})=\sum_{\ell} i^{\ell} \cdot \frac{f_{\ell}(r)}{r} Y_{\ell}^{0}(\hat{r})
$$

we obtain for the Yukawa kernel given in (6) above:

$$
\begin{aligned}
\frac{\hbar^{2}}{2 m}\left\{\frac{d^{2}}{d r^{2}}-\frac{\ell(\ell+1)}{r^{2}}\right\} f_{\ell}^{2}(r)+ & E_{C M^{2}} f_{\ell}(r)=\frac{1}{\beta^{2}} \int_{0}^{\infty} \sqrt{r r^{\prime}} d r^{\prime} \frac{U_{N}(r)+U_{N}\left(r^{\prime}\right)}{2} \\
& \times I_{\ell+1 / 2}\left(r_{<} / \beta\right) K_{\ell+1 / 2}\left(r_{>} / \beta\right) f_{\ell}\left(r^{\prime}\right) .
\end{aligned}
$$

Here $I_{n}$ and $K_{n}$ are modified Bessel functions. ${ }^{7}$

If instead we assume that the kernel can be separated into sub-kernels which describe the radial and angular parts of the non-locality, of the form:

$$
\begin{aligned}
& h\left(\vec{r}, \vec{r}^{\prime}\right)=k\left(\hat{r}, \hat{r}^{\prime}, \alpha\right) \times g\left(r, r^{\prime}, \beta\right) \quad \text { with } k\left(\hat{r}, \hat{r}^{\prime}, \alpha\right)=\frac{\alpha}{4 \pi} \frac{\exp \left(\alpha \hat{r} \cdot \hat{r}^{\prime}\right)}{\sinh (\alpha)} \\
& \text { and } g\left(r, r^{\prime}, \beta\right)=\frac{\delta\left(r-r^{\prime}\right)}{r r^{\prime}} .
\end{aligned}
$$

then we obtain a reduced Schrödinger equation of the form:

$$
\frac{\hbar^{2}}{2 m} \cdot\left\{\frac{d^{2}}{d r^{2}}-\frac{\ell(\ell+1)}{r^{2}}\right\} f_{\ell}(r)+\left\{E_{C M}-U_{N}(r) \frac{\alpha}{\sinh (\alpha)} \sqrt{\frac{\pi}{2 \alpha}} I_{\ell+1 / 2}(\alpha)\right\} f_{\ell}(r)=0
$$

This equation' may be interpreted as indicating that when we separate the radial and angular non-locality and suppress the radial effects, the result is an $\ell$ dependent effective potential.

Another way of separating the angular and radial nonlocal ranges is to modify the Perey-Buck kernel to that of an elliptical Gaussian:

$$
h\left(r, r^{\prime}\right)=\left[\lambda^{2} /(\beta \sqrt{\pi})^{3}\right] \exp \left\{-\lambda^{2} / \beta^{2}\left[r^{\prime 2}\left(1-\cos ^{2}\left(\hat{r} \cdot \hat{r}^{\prime}\right)\right]-1 / \beta^{2}\left[r-r^{\prime} \cos \left(\hat{r} \cdot \hat{r}^{\prime}\right)\right]^{2}\right\}\right.
$$

and we note that this becomes a conventional Gaussian when $\lambda=1$. When this kernel is used the radial Schrödinger equation has a form similar to Eq. (12) but with a different $\ell$-dependent potential of the form:

$$
\bar{U}_{\ell}\left(r^{\prime}, r^{\prime}\right)=U_{N}\left[\left(r^{\prime}+r^{\prime}\right) / 2\right] \times h_{\ell}\left(r^{\prime}, r^{\prime}\right)
$$


where

$$
\begin{aligned}
& h\left(r, r^{\prime}\right)=\left[\lambda^{2} / \beta \sqrt{\pi}\right] \exp \left\{-\left[z+\left(r-r^{\prime}\right)^{2} / \beta^{2}\right] 2 z\left[\sqrt{\pi / 2 z} I_{\ell+1 / 2}(z)\right]\right. \\
& z=2\left[r^{\prime}+r^{\prime 2}\left(\lambda^{2}-1\right)\right] / \beta^{2} \text { and } I_{\ell+1 / 2}(z) \text { is a modified Bessel function. }
\end{aligned}
$$

Again we note that when $\lambda=1$ the kernel becomes a spherical Gaussian and the above expression reduces to the Perey-Buck form.

(3) A Treatment of the Perey Effect to Order $B^{2}$

Finally we consider the effect of Perey damping on the wave functions. The local energy approximation used above, and found to work quite well for small non-local ranges, is an approximation whose derivation is given, for example, in Austern's book. ${ }^{8}$ However, this derivation treats some second order terms explicitly while neglecting others. It is therefore of interest to rederive this approximation so that all of the second order terms are retained. With this in mind we expanded all functions in the non-locality integral to second order. In this way an. approximation might be found which retains its validity for larger values of the non-local range. On performing this calculation it was found that when all second order terms are kept, the expression is by no means as simple as the LEA, and becomes a set of coupled non-linear equations. We start with:

and

$$
-\left(\hbar^{2} / 2 m\right) \nabla^{2} \times(\vec{r})+\left[\bar{U}\left(\vec{r}, \vec{r}^{\prime}\right) \times\left(\vec{r}^{\prime}\right) d^{3} r^{\prime}=E_{C M} \times(\vec{r}) \text { with } \bar{U}=U_{N}\left(\frac{r+r^{\prime}}{2}\right) h\left(\left|\vec{r}-\vec{r}^{\prime}\right|\right)\right.
$$

$$
x(\vec{r})=F(r) \chi_{L}(\vec{r}) \text { where the Perey function } F(r) \rightarrow 1 \text { as } r \rightarrow \infty \text {. }
$$

Here $x_{L}(\vec{r})$ is the local wave function which satisfies the local equation:

$$
\left\{-\left(x^{2} / 2 m\right) \nabla^{2}+U_{L}(r)-E_{C M}\right\} X_{L}(\vec{r})=0 .
$$

We wish to solve for the function $F(r)$ and the effective local potential $U_{\text {. }}(r)$ so that we can easily generate non-local wave functions from the corresponding local wave functions calculated with $\mathrm{U}_{\mathrm{L}}(r)$.

Solution of the above equations to second order in $B$ reduces to the following equations for $F(r)$ and $U_{L}(r)$ :

$U_{L}(r)=\cdot\left(x^{2} / 2 m\right) \frac{\nabla^{2} F}{F}+$

$\left[U_{N}(r)+\beta^{2} / 4\left(\frac{1}{4} \frac{d^{2}}{d r^{2}} U_{N}+\frac{U_{N}}{F} \frac{d^{2}}{d r^{2}} F+\frac{1}{F} \frac{d U_{N}}{d r} \frac{d F}{d r}+\frac{2 U_{N}}{F r} \frac{d F}{d r}+\frac{1}{r} \frac{d U_{N}}{d r}\right)\right] \exp \left(-k^{2} \beta^{2} / 4\right)$

In $F=\frac{1}{2} \int_{\infty}^{r} \frac{d U_{N}}{d r} d r\left[U_{N}-\frac{2 \hbar^{2}}{m \beta^{2}} \exp \left(k^{2} \beta^{2} / 4\right)\right]^{-1}$ with $\hbar^{2} k^{2}(r) / 2 m=E_{C M}-U_{L}(r)$.

We note that when we take $F=1$, Eq. (14) reduces to Eq. (5).. These equations can be solved by iteration, taking $F=1$ as a starting value, and it is expected that 
the convergence will be quite good. Thus, if there is need for a better approximation than the LEA for describing the. Perey damping and obtaining effective local potentials, this method would appear to provide a satisfactory approach. Extension of this technique to higher orders in $\beta^{2}$ is not feasible since the potential becomes $\ell$ dependent for order $\beta^{4}$.

We intend to apply some of the above techniques to heavy ion reaction calculations to investigate whether "elliptical" nonlocality or large non-local ranges can account for some of the discrepancies between heavy ion cross section data and DWBA calculations.

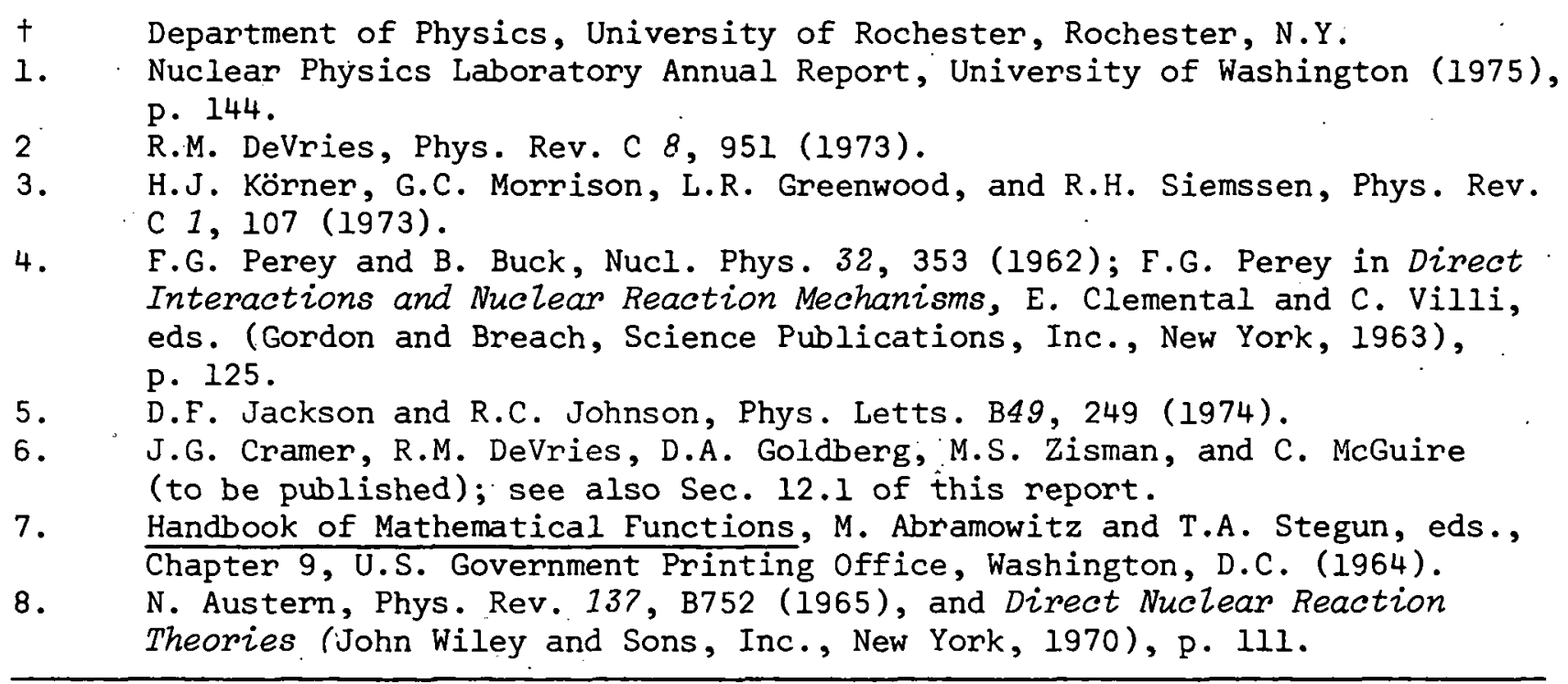




\section{HEAVY ION DEEPLY INELASTIC AND FUSION REACTIONS}

13.1 X-ray Technique for Measurement of Heavy Ion Nuclear Charge Distributions

P. Dyer, J. Pedersen, and R. Vandenbosch

We have continued studies of the new particle-x-ray coincidence technique for measuring nuclear charge distributions of reaction products from very heavy ion reactions. 1 This work is of particular interest to studies of the reaction mechanism for deeply inelastic scattering. 2 The detection system, based on observation of $K \times$ rays characteristic of the nuclear charge of a particular reaction product, consists of a radiaton fnil, followed by a tranemiesion-mounted silicon particle detector and an intrinsic Ge $x$-ray detector (see Fig, 13.1-1). If $a, k$ vacancy is created when the heavy in passes through the radiator foil, a characteristic $K \times$ ray may be observed in coincidence with the particle.

The detection system has been tested using $700-\mathrm{MeV}{ }_{36}^{84} \mathrm{Kr}$ and $1100-\mathrm{MeV}{ }_{54}^{136} \mathrm{Xe}$

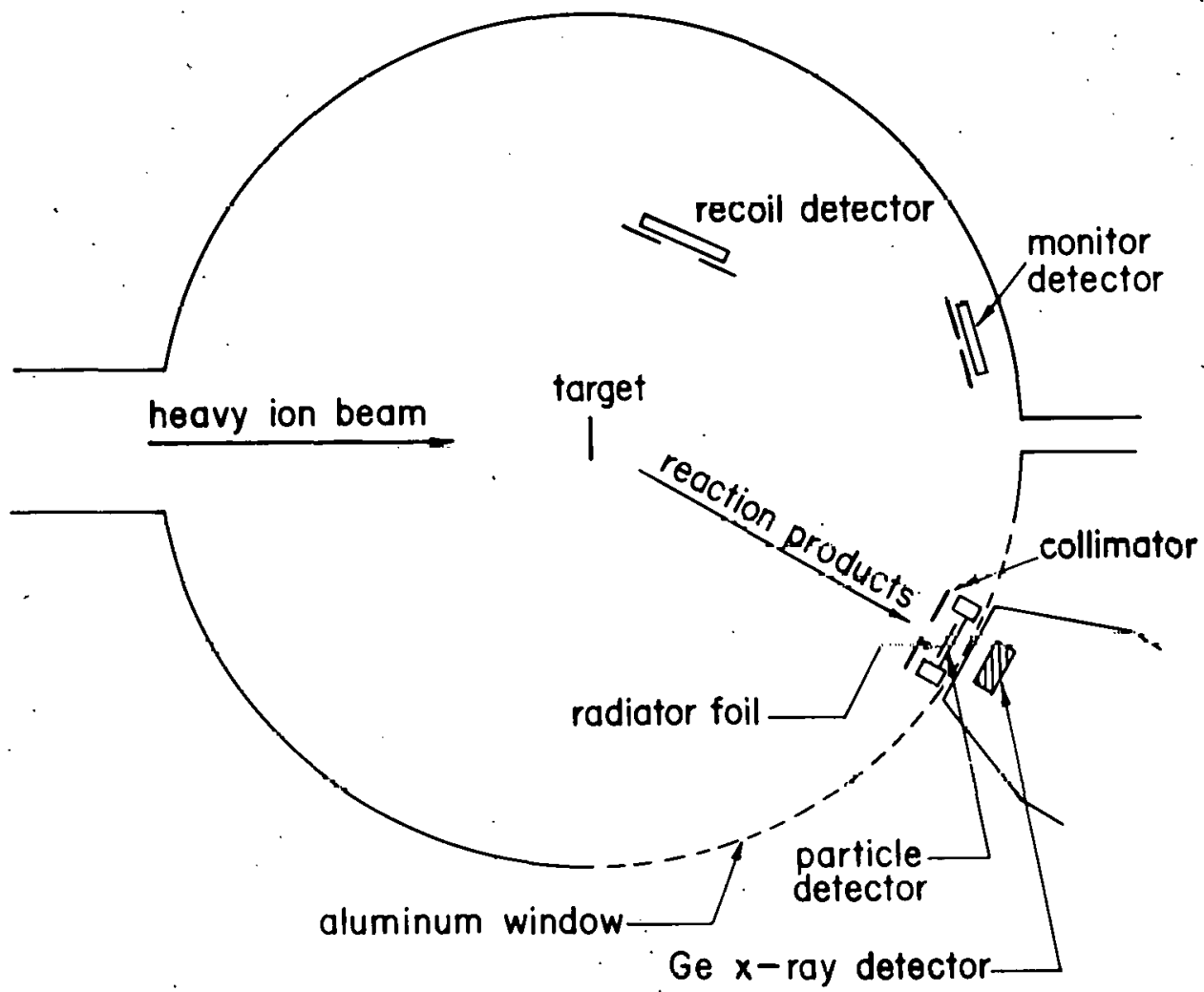

Fig. 13.1-1. Schematic diagram of the apparatus for identifying nuclear charges of reaction products by characteristic $K \times$ rays produced by atomic collisions in the radiator foil. 

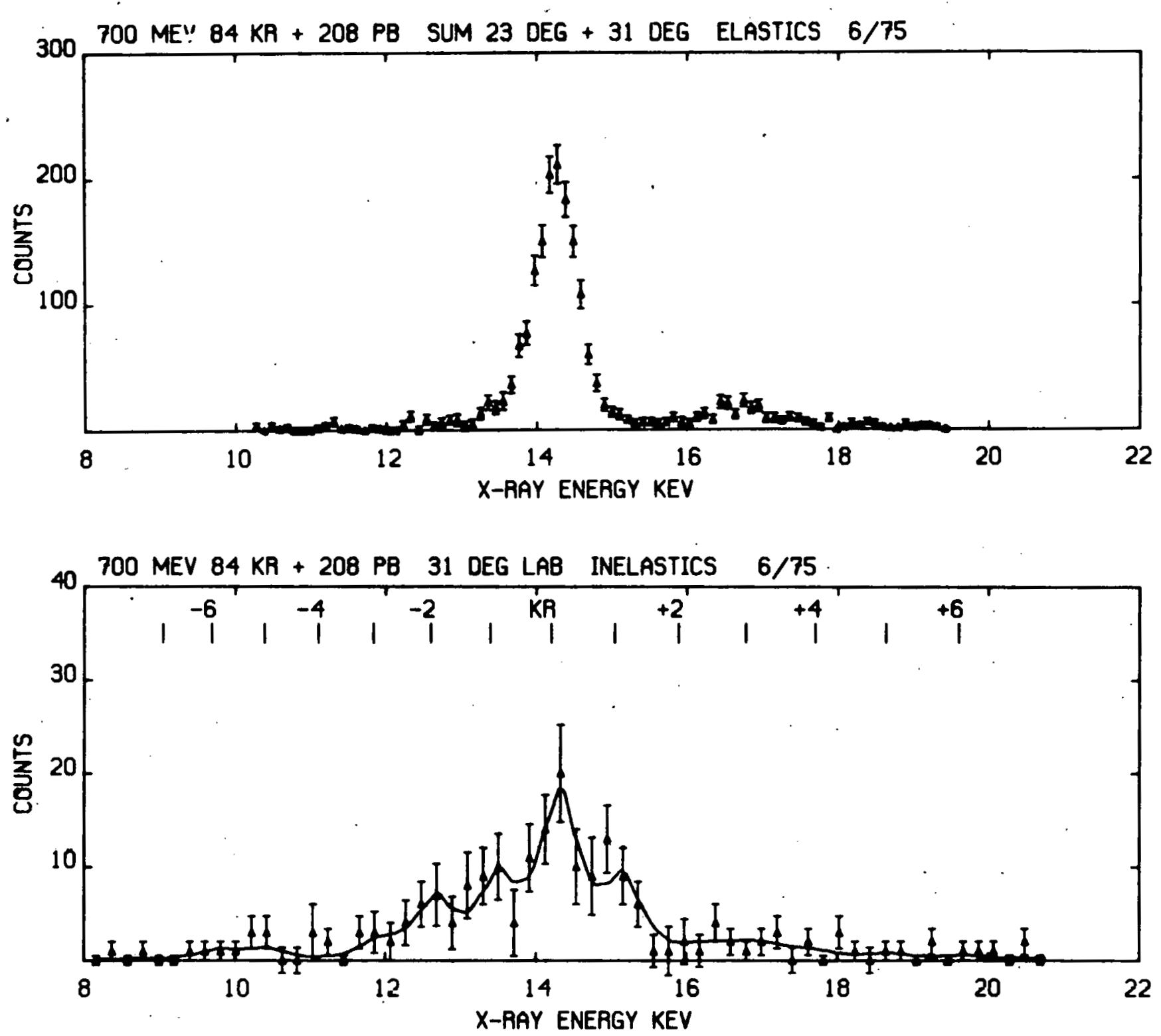

Fig. 13.1-2. The top figure shows the spectrum of $x$ rays coincident with elastically scattered particles from $700-\mathrm{MeV} 8{ }^{4} \mathrm{Kr}+208 \mathrm{~Pb}$. It is obtained by summing spectra taken at $23^{\circ}$ and $31^{\circ}(1 \mathrm{ab}) . K_{\alpha}$ and $K_{\beta} \times$ rays characteristic of $K_{r}$ are ubserved. The bottom figure shows the spectrum of $x$ rays coincident with deeply inelastic reaction products at the peak of the angular distribution, $31^{\circ}(1 a b)$. The positions of $K_{\alpha} \times$ rays from $K r$ and elements of higher and lower $Z$ 's are indicated. The solid line is a preliminary fit to the data obtained by varying the amplitudes of 14 lines and a constant background, where the lineshape is obtained from the elastic data shown in the top figure. Doppler shifts are taken into acoount. 
beams incident on ${ }^{208} \mathrm{~Pb}$ targets at the LBL SuperHILAC. For each of these reactions, spectra were taken at the peak of the deeply-inelastic-scattering angular distribution, and on each side of the peak. A new scattering chamber, constructed with a thin aluminum window on one quadrant to transmit $x$ rays, was used. The Xe data was acquired with the new intrinsic Ge detector purchased from LBL. The rate at which data can be acquired is limited by large singles $x$-ray rates from the target (rates which are enhanced over the time-averaged rates because of the low duty factor of the SuperHILAC) and by the solid angle which can be subtended by the $x$-ray detector relative to the radiator foil. X-ray lines exhibit greater Doppler broadening for close geometries. In the Xe experiment, we moved the $x$-ray detector off the target-particle-detector axis, so that $x$-rays from the target were not detected. A recoil detector was added to the detection configuration of the Xe experiment, so that kinematic coincidence data could be acquired simultaneously. This made it possible to measure both the charge and the mass distribution of the reaction products.

The spectra of $x$ rays coincident with elastically scattered ${ }^{84} \mathrm{Kr}$ and with deeply inelastic reaction products are shown in Fig. 13.1-2. In the $\mathrm{Kr}$ and in the $\mathrm{Xe}$ experiment, we have been successful in observing $\mathrm{x}$ rays characteristic of reaction products having a range of $Z^{\prime} ' s$, but the atomic cross sections were not as large as anticipated, so that the detection system has a very low efficiency, especially for reaction products having $\mathrm{Z} \rightarrow 50$. In addition, in the Xe experiment we observed more $x$ rays from internal conversion of the excited reaction products than from vacancies produced by atomic collisions in the radiator foil. It is then not possible to extract an accurate charge distribution from the energy spectrum because of unknown nuclear contributions. Because it has recently been possible to separate adjacent $Z$ reaction products up to $Z=54$ with $\mathrm{dE} / \mathrm{dx}$ gas ionization counters, 3 the $x$-ray method is not presently a competitive technique.

It should be noted, however, that the $\mathrm{x}$-ray cross section (and thus the detection efficiency) is still increasing rapidly with reaction-product energy at the highest available SuperHILAC energies, so that the technique may prove of value to experiments conducted on some future generation of heavy ion accelerators. As $x$-ray detectors that are radially position-sensitive may become available within a few years, it might be possible to eliminate the Doppler broadening problem.

Before ending this program, we will measure a series of x-ray production cross sections that are required for a tinal evaluation of the usetulness of the terhninue.

1. Nuclear Physics Laboratory Annual Report, University of Washington (1975), ค. 109 .

2. See Sec. 13.5 of this report.

3. L.G. Moretto, private communication. 
13.2 A New Technique for Nuclear Lifetime Measurement in the Attosecond Range

D. Burch and P. Dyer

Measurements of nuclear Iffetimes are a powerful means of studying nuclear structure and nuclear reaction mechanisms. Thus far, the only direct method for measuring lifetimes shorter than $10^{-15} \mathrm{sec}$ has been the crystal blocking technique. Recent studies at NPL show great promise for a new technique, applicable to measurement of lifetimes of the order of $10^{-16} \mathrm{sec}$ for light nuclei, ranging to $10^{-18}$ sec for heavy nuclei.

In simple terms, the technique is to produce a nuclear and an atomic excited state simultaneously and to observe which decays first. 1 This is done by looking at characteristic $\mathrm{K} \times$ rays in coincidence with the product of the nuclear decay. The unknown nuclear lifetime can then be related to the relatively wellknown atomic lifetime. Lifetimes of compound nuclei, residual nuclei, and particle-unstable states produced by $\beta$ decay might be measured by this technique.

As an example, we have studied the feasibility of measuring compound nuclear lifetimes by bombarding a $\mathrm{Sn}$ target with a $63-\mathrm{MeV} 160$ beam. In this case we are looking for events in which the incident 160 particle produces a $K$ vacancy in the atomic shell before forming the compound nucleus cerium. Decay of the compound nucleus is measured by detecting an evaporated proton (or alpha particle); decay of the atomic state is measured by the $K \times$ ray characteristic of the compound nucleus or the residual nucleus. If the nuclear state decays first, coincident La (or $\mathrm{Ba}$ ) $x$ rays are observed; if the atomic state decays first, coincident $\mathrm{Ce} \times$ rays are observed. The atomic lifetime is $5 \times 10^{-17} \mathrm{sec}^{2}$ the nuclear lifetime is expected to be less than $10^{-18} \mathrm{sec}$. The probability of K-shell ionization for the $16_{0}$ beam on the Sn target, $P_{K}(0) / 2$, is about 0.03 .3 The difficulty of this experiment lies in extracting a low yield of coincidence counts in the presence of high singles rates.

Figure 13.2-1 shows a spectrun of $\mathrm{x}$ rays in coincidence with light evaporated particles. These data were taken using the new intrinsic Ge $x$-ray detector purchased from the Lawrence Berkeley Laboratory. A copper absorber was placed in front of the $x$-ray detector to reduce the number of target $(S n) \times$ rays; a nickel foil in front of the particle detector stopped heavy ions. Lanthanum (and some barium) $x$ rays are observed, and an upper limit can be placed on the number of cerium $x$ rays. If all the La $K x$ rays come from vacancies produced by the incident 160 particles (an assumption which requires further experimental justification), we can conclude, on the basis of the ratio of Ce to La $x$ rays, that the compound nuclear lifetime is less than $5 \times 10^{-18} \mathrm{sec}$. An absolute measurement of the number of $\mathrm{Ce} x$ rays is required if there are sources of La $x$ rays of unknown magnitude, such as internal conversion of ta nuclear states or $\mathrm{K}$ vacancy production by the emergent proton or by the recoil La-Sn interaction.

In the case of $63-\mathrm{MeV}{ }^{16} \mathrm{O}+\mathrm{Sn}$, the ratio of the nuclean to the atomic lifetime is expected to be small. This ratio increases for higher-Z targets at 160 bombarding energies near the coulomb barrier. Compound nuclear $x$ rays (and thus finite lifetimes) should thus be observable with a higher-energy (3-stage) 160 beam incident on a higher $\mathrm{Z}$ target. Apparatus for achieving closer detection 


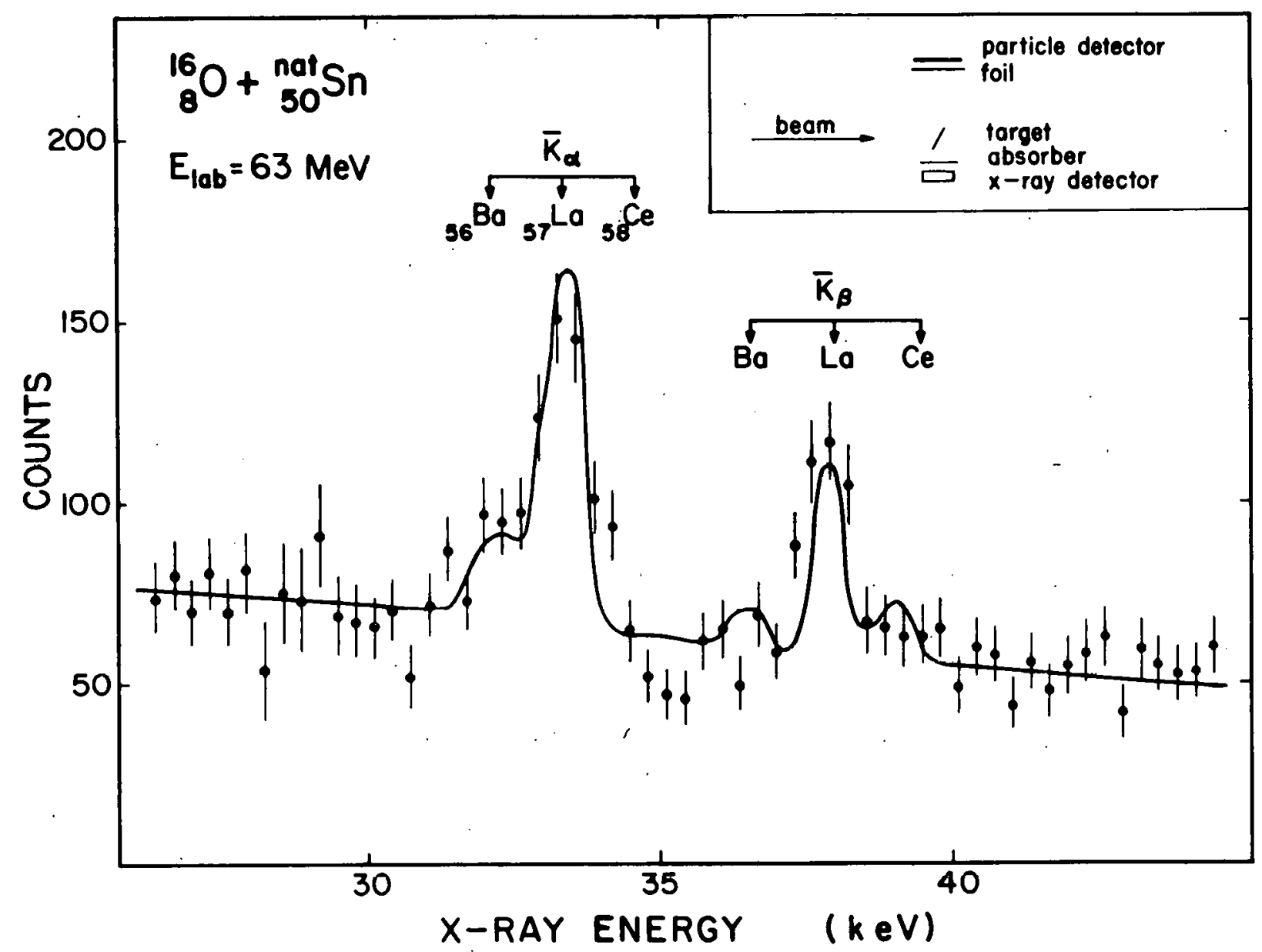

Fig. 13.2-i. Ennergy spectrum of $x$ rays coincident with light evaporated particles. The accumulation time was 7 hours. Energies characteristic of the compound and residual nuclei are indicated. The solid line is a fit obtained by varying the amplitudes of three lines and a linear background, where the lineshape was measured by bombarding a cerium oxide target with the $16_{0}$ beam. The inset shows the detection geometry.

geometry and $\mathrm{dE} / \mathrm{dx}$ particle identification is now under construction.

Feasibility studies have also begun for measuring widths of particleunstable states of ${ }^{32} \mathrm{~S}$ via the ${ }^{2} 8 \mathrm{Si}(\alpha, p)^{31_{\mathrm{P}}}$ reaction, and of states of ${ }^{41_{S C}}$ (particularly the lowest $T=3 / 2$ state) via $\beta$-delayed proton emission from

$$
41_{\mathrm{Ti}} \stackrel{\beta^{+}}{\rightarrow} 41_{\mathrm{Sc}} \text {. }
$$

1. P.C. Gugelot, Proc. of the Conf. on Direct Interactions and Nuclear Reaction Mechanisms, Padua, 1962, p. 382.

2. Walter Bambynek et al., Rev. Mod. Phys. 44, 716 (1972).

3 . See Sections 5.5 and 5.6 of this report. 
13.3 Study of Excitation Functions for ${ }^{16} \mathrm{O}$ and ${ }^{18} \mathrm{O}$ Induced Reactions on ${ }^{12} \mathrm{C}$

K.G. Bernhardt, H. Bohn, Y-d Chan', J.G. Cramer, L. Green, R. Sielemann, and $R$. Vandenbosch

At the present time very little information is available about the detailed energy dependence of heavy ion induced fusion cross sections over a large bombarding energy range. Two different measurement techniques have been applied for the detection of the residual nuclei: (1) $\mathrm{dE} / \mathrm{dx}-\mathrm{E}$ telescope systems, together with time of flight techniques; and (2) detection of characteristic $\gamma$-rays with $\mathrm{Ge}(\mathrm{Li})$ spectrometers. An example for the successful application of the first technique is the total fusion cross section study of the ${ }^{12} \mathrm{C}+160$ system between $E_{C M}=13 \mathrm{MeV}$ and $27 \mathrm{MeV}$ reported by $P$. Sperr et al. I The second method has been used to study fusion cross sections for various heavy ion systems.

In addition to the fact that experimental fusion cross sections provide information on the importance of such parameters as the critical radius, critical angular momentum, and interaction barrier, the recent observation 1 of unexpected structure in the energy dependence of the fusion cross section has led to increased interest in fusion cross sections. It is not known whether this kind of structure is unique to the ${ }^{12} \mathrm{C}+160$ system, or is exhibited also by other systems in this mass region. It is also not yet clear if the oscillations in the total fusion cross section could be somehow related to the intermediate structures observed in particle decay to particular levels in several light heavy ion systems.

Because we are also interested in extending the present knowledge about intermediate structure in the $12 \mathrm{C}+160$ and $12 \mathrm{C}+18_{0}$ systems by studying heavier particle exit channels ( ${ }^{8} \mathrm{Be}$, see Sec. 14.2 of this report) and because we think that the measurement of fusion cross sections could be a complementary method to look at these systems, we decided to measure fusion cross sections for $12 \mathrm{C}+16_{\mathrm{O}}$ and $12 \mathrm{C}+180$.

We applied the second technique mentioned above for this purpose. Since the low lying level structure in most of the possible residual nuclei is known up to about $8 \mathrm{MeV}$ excitation energy and is fairly simple (in contrast for example to odd-A rare earth nuclei) the cross sections for their production can be obtained by integrating those measured $\gamma$-ray intensities which represent the last steps in the deexcitation process. The total cross section then results from summing up individual channels.

This method (together with particle- $\gamma$ coincidence techniques) has the potential for also providing information about the intrinsic $\gamma$-deexcitation modes of individual final nuclei, which could give important insight into the yrast level population pattern. In addition the recoil of a residual nucleus measured by the Doppler shift of the corresponding $\gamma$-ray transition can help to establish the reaction mechanism as for example distinguishing between mass transfer to the target nucleus from mass transfer to the projectile.

We measured $\gamma$-ray spectra as a function of bombarding energy for ${ }^{12} \mathrm{C}+{ }^{16} \mathrm{O}$ between $E_{C M}=6.5 \mathrm{MeV}$ and $32.0 \mathrm{MeV}$ and for $12 \mathrm{C}+18_{0}$ between $E_{C M}=6.1 \mathrm{MeV}$ and $32.0 \mathrm{MeV}$. Near the Coulomb barrier we measured in $\Delta E_{C M}=1.0 \mathrm{MeV}$ steps, above the 
Coulomb barrier in $\Delta E_{C M}=0.5 \mathrm{MeV}$ steps and above $E_{C M} \approx 23 \mathrm{MeV}$ in $\Delta E_{C M}=1.0 \mathrm{MeV}$ steps. The target consists of a layer of $112 \mu \mathrm{g} / \mathrm{cm}^{2} 12 \mathrm{C}$ evaporated onto a $92 \mathrm{mg} / \mathrm{cm}^{2}$ thick metallic Ta backing which was thick enough to stop the beam and the reaction products. This was necessary to avoid strong Doppler broadening of $\gamma$-transitions due to the recoil of the residual nuclei into vacuum, as was observed with a $100 \mu \mathrm{g} / \mathrm{cm}^{2}$ self-supporting ${ }^{12} \mathrm{C}$ target at $E_{C M}=12.9 \mathrm{MeV}$. The $\gamma^{-}$ rays were detected in a coaxial, $50 \mathrm{~cm}^{3} \mathrm{Ge}(\mathrm{Li})$ spectrometer ( $9.6 \%$ efficiency compared to a $3 \times 3$ inch $\mathrm{NaI}$ ), located at $90^{\circ}$ with respect to the beam axis and $10 \mathrm{~cm}$ from the target, which was located in a 10" D-shaped scattering chamber. 'l'he $\gamma$ ray energy spectra were stored in two modes: 4096 channels for the energy range of $0 \mathrm{MeV}-4 \mathrm{MeV}$ and 2048 channels for the range of $2 \mathrm{MeV}-8 \mathrm{MeV}$. The beam collimation system was placed $\approx \mathrm{I} \mathrm{m}$ in front of the target and consisted of two Au collimators (first: $\phi=4.8 \mathrm{~mm}$, second: $\phi=6.4 \mathrm{~mm}$ (clean up)). To examine the background situation with the actual setup we accumulated $\gamma$-ray spectra while sending a $E_{\text {lab }}=30 \mathrm{MeV} 100$ beam through an empty target frame. The beall was then stopped in a Faraday cup located $6 \mathrm{~m}$ downstream from the target. The resulting $\gamma$-background is very low compared to the actual measurements done later and is mainly activation background. Only one stronger $\gamma$-line of $1460 \mathrm{keV}$ shows up, which is the $2^{+}-0^{+}$transition in $40 \mathrm{Ar}$ resulting from the $\beta^{+}$-decay of $40 \mathrm{~K}$.

Because we need to know the effective target thickness to obtain absolute cross sections, we compared at certain bombarding energies the yields of special $\gamma$-ray reaction lines obtained with the backed target with those obtained with a thin self-supporting ${ }^{12} \mathrm{C}$ foil, the thickness of the latter being determined separately by measuring an elastic scattering angular distribution at $\mathrm{E}_{1 \mathrm{ab}}\left(16_{0}\right)=$ $30 \mathrm{MeV}$ in the 60" scattering chamber. This comparative method requires an absolute normalization which was achieved by electrically connecting the Faraday cup with the scattering chamber containing the backed target, which in turn was isolated from the rest of the beam line system.

Possible carbon buildup on the backed target during the later runs was checked about every six runs by golng back to a fixed bombarding energy $\left(E_{1 a b}\left(16_{0}\right)=\right.$ $45 \mathrm{MeV})$ and comparing the intensity of special reaction lines with those of the Coulomb excitation lines of ${ }^{181} \mathrm{Ta}$. An additional advantage of using the thick $\mathrm{Ta}$ backing is the fact that the ratio of the intensity of a given Coulomb excitation line to the integrated beam charge should show, at least for bombarding energies below the Coulomb barrier for $16_{0}+181_{\mathrm{Ta}}\left(\mathrm{E}_{\mathrm{CB}}^{\mathrm{CB}} \approx 78 \mathrm{MeV}\right.$ ), a smooth dependence as a function of bombarding energy. The $\gamma$-raby intensities were obtained by using the peak shape and background fitting program GELIFIT (see Sec. 4.6 of this report).

Typical y-ray energy spectra taken at $E_{C M} \approx 9.5 \mathrm{MeV}$ are displayed in Fig. 13.3-1 for the $12 \mathrm{C}+160$ and $12 \mathrm{C}+180$ entrance channel systems. The expertations about the general form of the $\gamma$-ray energy spectra are confirmed: For a given bombarding energy only a few strong $\gamma$-ray lines corresponding to the last deexcitation steps in the different final nuclei are showing up. Most of them are well separated in energy, therefore allowing an accurate intensity extraction. The $\gamma$-ray line shape is in principle symmetric and narrow in width. A few lines exhibit broader shoulders on both sides of the center. This effect is attributed to the slowing down process of the recoiling final nuclei in the Ta backing and is dependent on the intrinsic lifetime of the $\gamma$ emitting state and on the feeding time to this state. No important reaction line seems to lie near the strong 


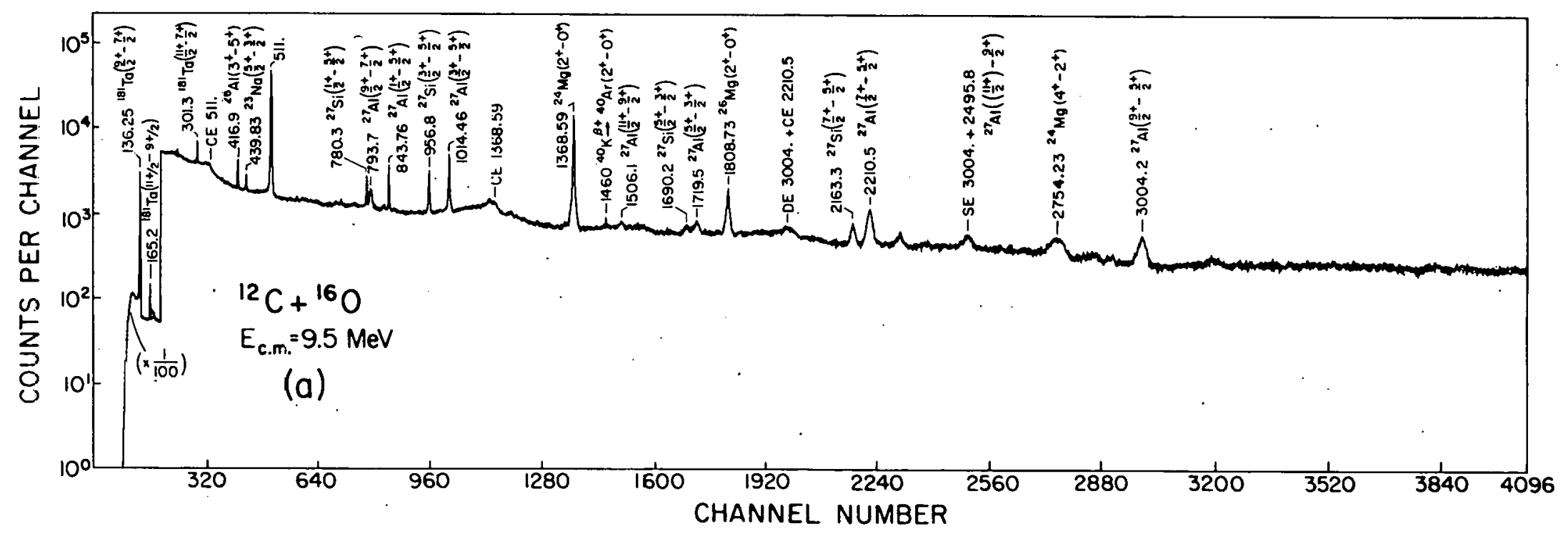

$\stackrel{\rightleftarrows}{\rightleftarrows}$

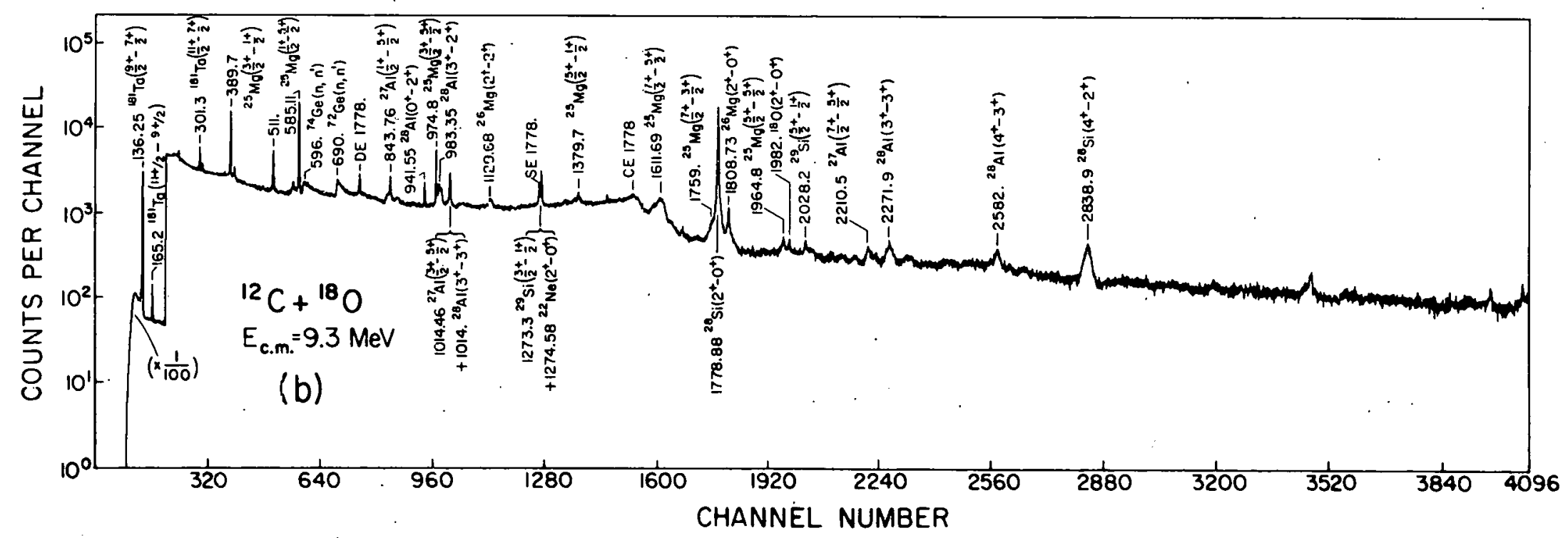
Fig. 13.3-1. Y-ray energy spectra for the ${ }^{12} \mathrm{c}+{ }^{16} \mathrm{O}(\mathrm{a})$ and ${ }^{12} \mathrm{C}+{ }^{18} \mathrm{O}(\mathrm{b})$ systems at $\mathrm{E}_{\mathrm{CM}} \approx 9.5 \mathrm{MeV}$.
I.jentified lines are indicated. 


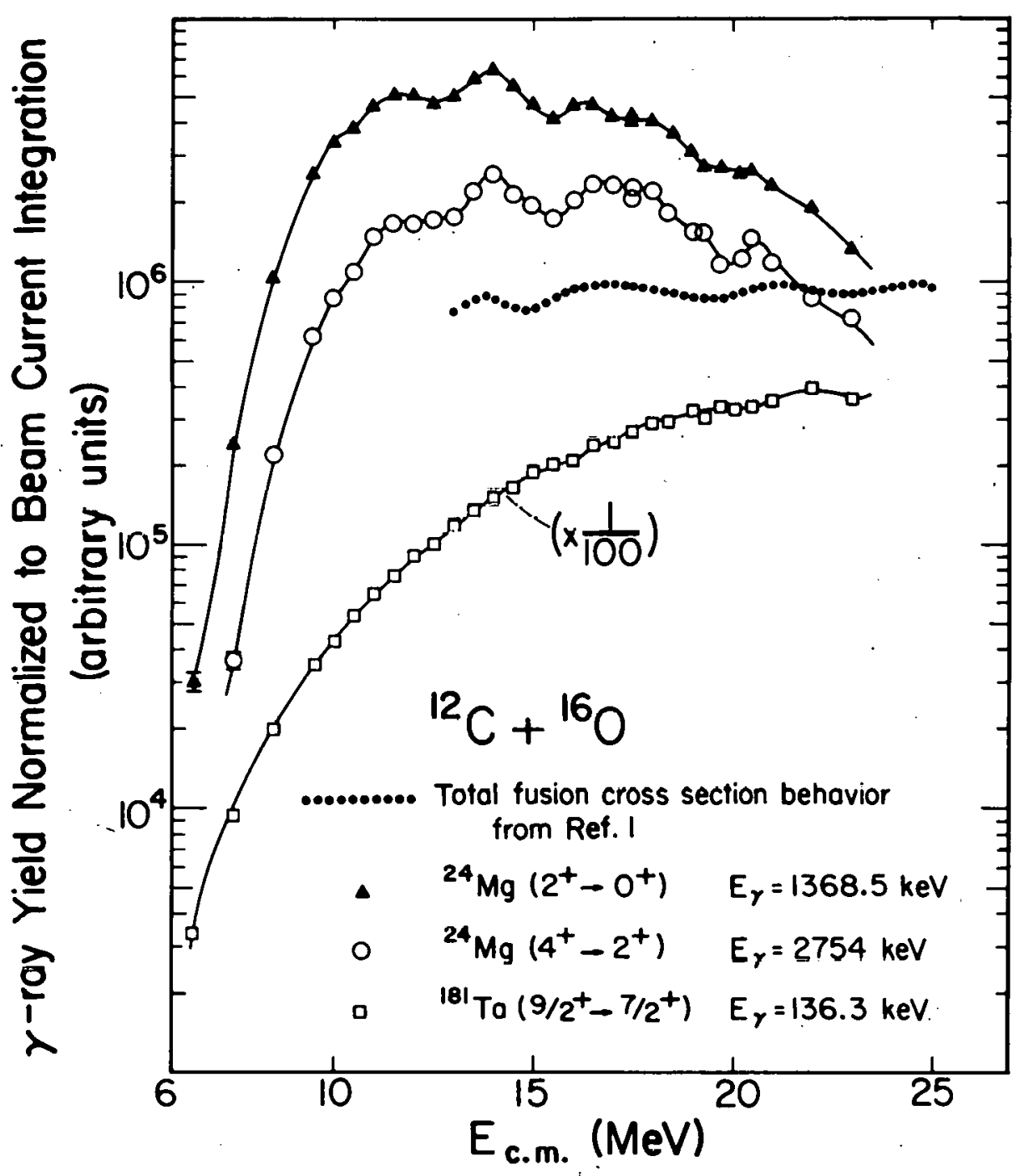

Fig. 13.3-2. Relative cross section behavion of the $2^{+\rightarrow 0^{+}}$and $4^{+} \rightarrow 2^{+} \gamma$-ray transition intensity in ${ }^{24} \mathrm{Mg}$ as a function of the center of mass energy for the $12 \mathrm{C}+$ 100 system, compared to the total fusion cross section data of Ref. 1. Also indicated is the ${ }^{181} \mathrm{Ta}$ Coulomb excitation yield to demonstrate the quality of the beam current normalization.

Coulomb excitation lines of ${ }^{181} \mathrm{Ta}$. Inelastir exritations due to the ${ }^{12} \mathrm{C}$ targrt. are also observed. For $16_{0}$ the $3 \rightarrow 0^{+}$transition of $6.13 \mathrm{MeV}$ could be easily identified in the compressed spectrum ( $2 \mathrm{MeV}-8 \mathrm{MeV}$ ) via the energies of the photopeak and the first and second escape peaks. For 180 the $2^{+} \rightarrow 0^{+}$transition $\left(E_{\gamma}=1.98 \mathrm{MeV}\right.$ ) could also be established (there is no previous experimental information available on the inelastic channels of the $12 \mathrm{C}+180$ system). The excitation of the $2^{+}$state at $4.43 \mathrm{MeV}$ in the target nucleus ${ }^{12} \mathrm{C}$. has not been observed yet. At the highest bombarding energies several $\gamma$-ray lines are observed which cannot be attributed to fusion-type reactions but could instead belong to residual nuclei populated via few nucleon transfer reactions. This seems to be 
especially true for the ${ }^{12} c+{ }^{18} 0$ system where several $\gamma$-ray lines could be associated-with one and two neutron transfer reactions to the ${ }^{12} \mathrm{C}$ target and also to the ${ }^{181}$ Ta backing.

The complete identification of the $\gamma$-ray lines and the cross section extraction from their intensities is presently under way. In order to get a first impression about the quality of our data we compare for the ${ }^{12} \mathrm{C}+16_{0}$ system our relative cross section behavior for producing ${ }^{24} \mathrm{Mg}$-- obtained from the ratio of the intensity of the $2^{+} \rightarrow 0^{+}$transition $\left(E_{\gamma}=1.37 \mathrm{MeV}\right)$ in ${ }^{24} \mathrm{Mg}$ to the integrated charge -- with the total fusion cross section excitation function of Sperr et al. ${ }^{1}$ in Fig. 13.3-2. In addition we included the $4^{+}+2^{+}$transition $\left(E_{Y}=2.75 \mathrm{MeV}\right)$ in

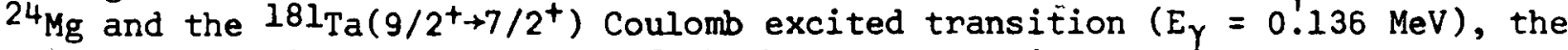
latter to confirm the accuracy of the beam current.integration.

$\cdot \quad \therefore$ Several interesting things can be concluded beyond the work of Ref. I:

1. : :

1. The cross section oscillations are not limited to the total fusion cross section but rather show up in individual exit channels.

2. The amplitude of the oscillations can be more pronounced in some particular exit channels compared to the total fusion cross section behavior.

3. The oscillations seem to originate primarily in the decay to states with higher angular momentum.

Statistical model calculations show that the alpha decay channel feeding ${ }^{24} \mathrm{Mg}$ is predominantly fed by those compound states having the largest . angular momenta, with neutron and proton decay. being more likely for the lower angular momenta states.

We hope to be able to further clarify the origin of these oscillations. after analys is of the $\gamma$-ray lines from other exit channels.

1. P. Sperr, S. Vigdor, Y. Eisen, W. Henning, D.G. Kovar, I'.K. Ophel, and B. Zeidman, Phys. Rev. Lett. 36, 405 (1976).

13.4 Deeply Inelastic Scattering of ${ }^{35} \mathrm{Cl}$ by ${ }^{59} \mathrm{Co}$

R. Bangert, J.E. Bussoletti, P.A. Dickey, M.B. Tsang, and R. Vandenbosch

We have initiated a study of the ${ }^{35} \mathrm{Cl}+{ }^{59} \mathrm{Co}$ reaction. Our primary interest is in the deeply inelastic scattering and fusion-fission reactions. We have made measurements in two different geometries thus far. The first consisted of a counter telescope on one side of the beam and an array of recoil detectors on the other side of the beam. Both counter-telescope singles events and coincidence events involving any of the recoil detectors were event-recorded on magnetic tape. In the second experiment the $5 \mu-\Delta E$ detector was separated from the $E$ detector of the telescope to make a time-of-flight spectrometer. The mass resolution was 
approximately 1.8 mass units. The bombarding energy was $121.6 \mathrm{MeV}$.

The zero-angular-momentum fission barrier for the compound system is very high (50-60 MeV) so that the only fission events expected are those from high angular momentum compound states. The formation of these states depends on the extent to which deeply inelastic scattering competes with compound nucleus formation for the lange partial waves. We see considerable yield for deeply inelastic events with kinetic energies comparable to the Coulomb barrier in the exit channel. Thus far we see very few events which might be fusion-fission events.

$13.5 \mathrm{Kr}$ and $\mathrm{Xe}$ Induced Reactions

T.D. Thomas ${ }^{\dagger}$, R. Vandenbosch, and M.P. Webb

We have extended our previous studies 1 of deeply inelastic scattering in very heavy systems to include the ${ }^{86} \mathrm{Kr}+{ }^{139} \mathrm{La}, 136 \mathrm{Xe}+18 \mathrm{I}_{\mathrm{Ta}}$ and ${ }^{136} \mathrm{Xe}$ to $208 \mathrm{~Pb}$ systeils. In contrast to lighter systems in which compound nuclear processes comprise a significant fraction of the total reaction cross section, $K r$ induced reactions on very heavy targets are found to be dominated by a direct reaction mechanism. The reaction products show near-complete equilibration of the incident kinetic energy yet very little mass transfer. The large coulomb repulsion in the entrance channel was shown ${ }^{1}$ to have important consequences on the subsequent interactions. The $\mathrm{Kr}+\mathrm{La}$ system was studied to see if a reduction in the Coulomb barrier yielded any changes in the kinds of reactions observed in previous $\mathrm{kr}$ induced reactions.

${ }^{86} \mathrm{Kr}$ beams from the SuperHILAC accelerator of the Lawrence Berkeley Laboratory were used to bombard $300 \mathrm{\mu g} / \mathrm{cm}^{2} 139 \mathrm{LaF}_{3}$ targets. Energy spectra were obtained with solid state surface barrier detectors. Unlike $\mathrm{Kr}$ bombardments of heavier targets in which only a single group of reaction products is in evidence, the reaction products in this system separate into two distinct components as shown by the energy spectra in Fig. 13.5-1. The lower energy component remains nearly a constant amount of energy removed from the elastic peak at values indicative of complete damping (i.e., conversion of all the available kinetic into intemal degrees of freedom) of the incident.kinetic energy. The high energy component shows a distribution of kinetic energies only partially damped with the amount of damping increasing with decreasing angle in a monotonic fashion. At the most forwand angle the kinetic energies of the two components overlaps to a large degree so that all the reaction products in this system at forward angles are nearly completely damped.

Angular dictributions have heen sonstruited fur lie two componenes of reaction products for those angles where they could be separated. The results are shown in Fig. 13.5-2. The partially damped component displays a bell-shaped angular distribution (Fig. 13.5-2a) characteristic of the deeply inelastic events in heavier systems. The angular distribution peaks at 8-10 degrees forward of the grazing angle which was determined to be $39^{\circ}$ (c.m.) from elastic scattering. The angular distribution of the lower energy, completely damped component shows a strikingly different behavior (Fig. 13.5-2b). This pronounced forward peaking followed by a rapid and smooth decrease in cross section with angle (and different from a pure $1 / \sin \theta$ type angular distribution) may be consistent with the 


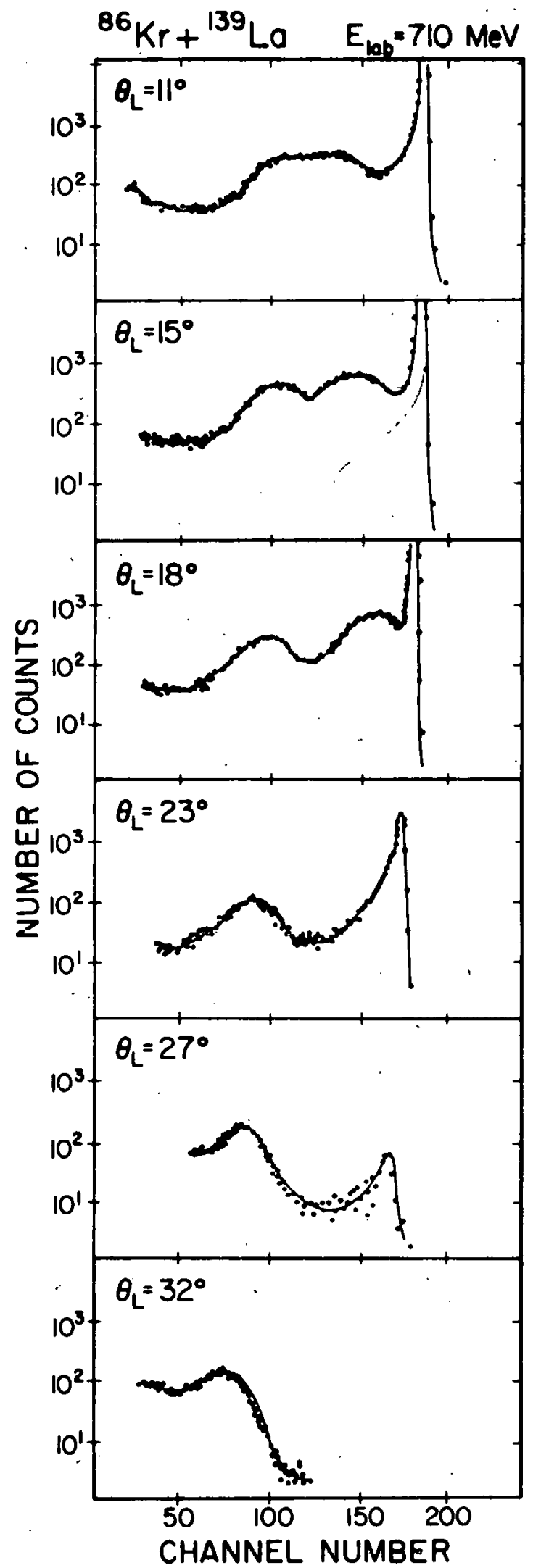

Fig. 13,5-1. Singles energy spectra for $86 \mathrm{Kr}+139 \mathrm{La}$ at $710 \mathrm{MeV}$. Only the shapes and not the absolute yields are indicated. The solid lines serve only to guide the eye. The spectra show all products entering the detector.

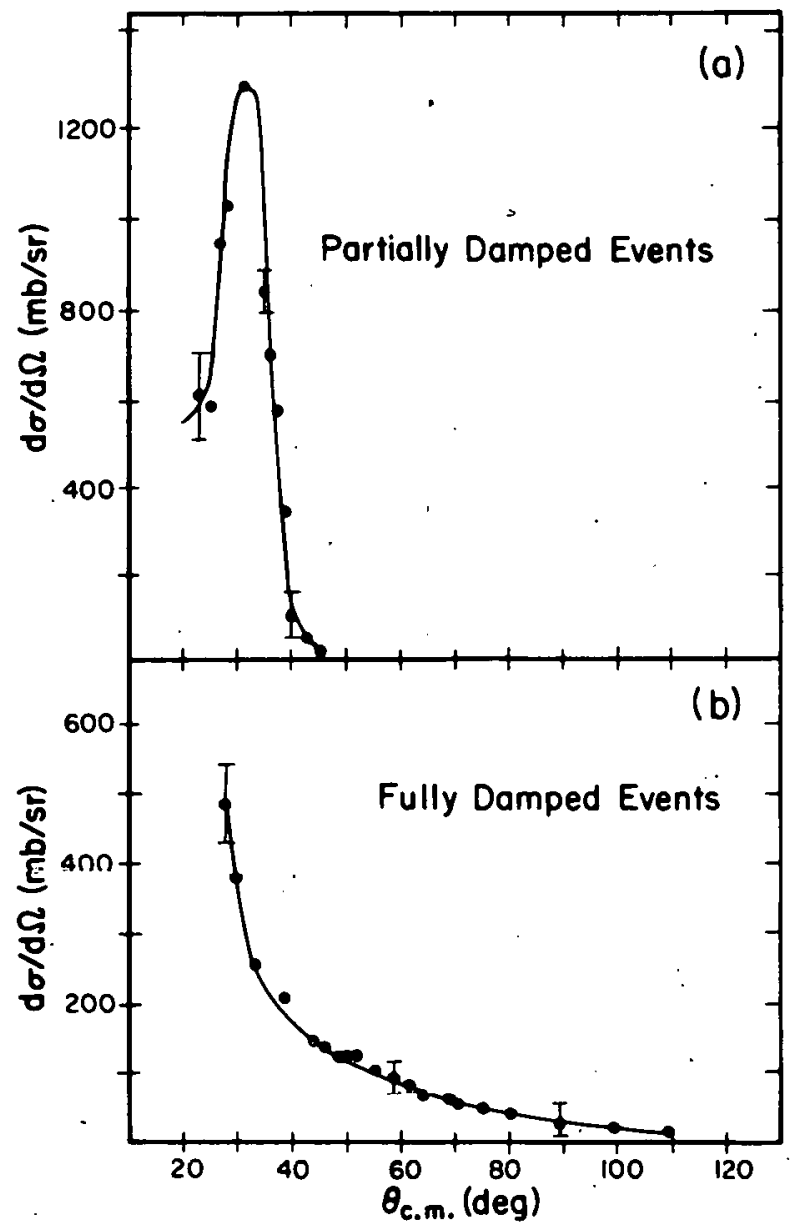

Fig. 13.5-2. Angular distributions for a) partially damped events and b) fully damped events. The solid lines serve only to guide the eye. 
idea of nuclear orbiting.

The angle integrated cross section for the partially damped component is $1200 \pm 150 \mathrm{mb}$ and that for the fully damped component is $1200 \pm 200 \mathrm{mb}$. The total reaction cross section determined from elastic scattering is $2830 \pm 150 \mathrm{mb}$. The difference between the sum of the above two components and the total reaction cross section may arise from a fusion-fission component which we are not able to uniquely identify.

A more complete overview of the various processes is provided by a contour plot of $\left(d^{2} \sigma / d E d \theta\right) c . m$, as shown in Fig. 13.5-3. The elastic peak dominates the energy-angle landscape. The partially damped events form a ridge running to lower energies and angles from the elastic peak while the fully damped events form a ridge running to higher angles at a constant energy. The second ridge is in agreement with the type of behavior expected to result from orbiting to negative angles.

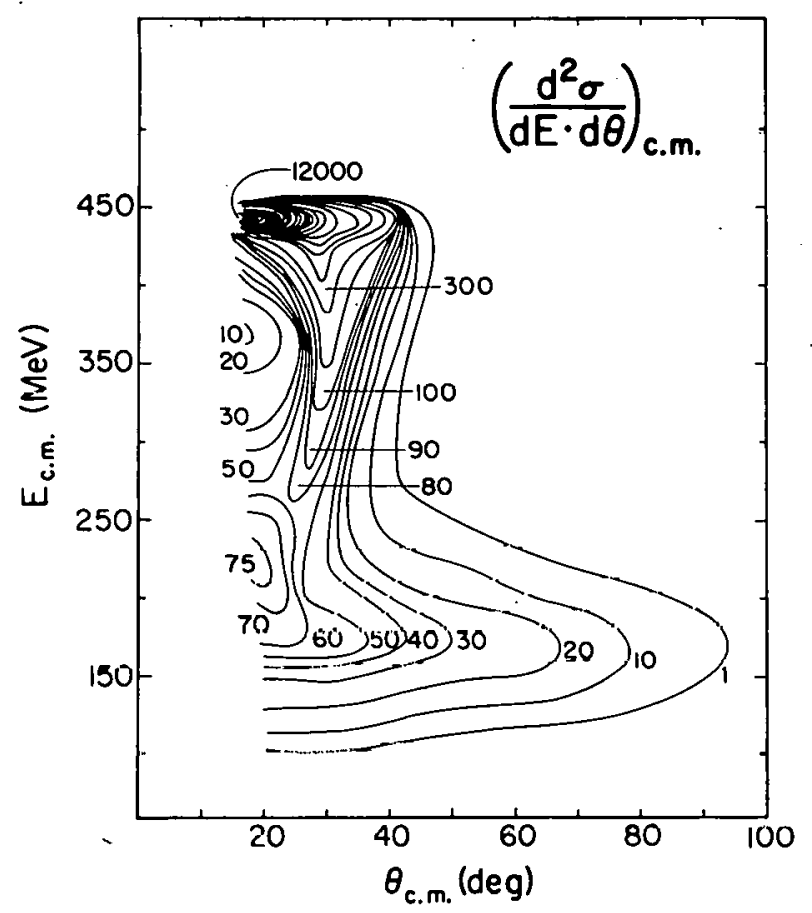

Fig. 13.5-3. Contour plot $\left(d^{2} \sigma / d E \cdot d \theta\right)_{c \cdot m}$. in $\mathrm{mb} / \mathrm{MeV} \cdot \mathrm{deg}$.

The data just discussed may be qualitatively understood by assuming that the higher partial waves contribute to the partially damped events with successively lower partial waves becoming increasingly damped until complete equilibration of the incident kinetic energy is achieved. The ovents which orbit to negative angles are comprised of low partial waves which are completely damped. From a study of the ${ }^{84} \mathrm{Kr}+208 \mathrm{~Pb}$ system it was found that the mechanism responsible for converting the kinetic energy into intemal degrees of freedom becomes less efficient as the bombarding energy is increased. The very dramatic existence of partially damped reaction products in the ${ }^{86} \mathrm{Kr}+{ }^{139} \mathrm{La}$ system suggests that, in addition, damping is less efficient with decreasing size of the interesting nuclei. The importance of orbiting may reflect the relative strength of the Coulomb and nuclear potentials felt during the collision. In very heavy systems where the Coulomb potential is very large orbiting is not a significant fraction of the cross section until a high enough energy is reached to produce sufficient interprenetration of the two nuclei. In lighter systrmn where fusion-fission iv dominant, the Coulomb potential is too small. Thus, orbiting may be important only in a restricted range of $\mathrm{z}_{1} \mathrm{Z}_{2}$ product end energy.

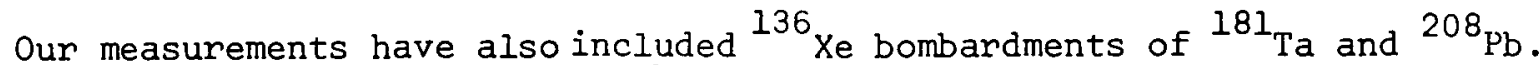
A $100 \mu \mathrm{g} / \mathrm{cm}^{2} 208 \mathrm{~Pb}$ and a $430 \mu \mathrm{g} / \mathrm{cm}^{2} 181_{\mathrm{Ta}}$ target were bombarded with a $1120 \mathrm{MeV}$ $136 \mathrm{Xe}$ beam. Energy spectra obtained at a number of different angles are shown in Fig. 13.5-4. For angles forward or backward of the angle corresponding to the peak of the angular distribution, the non-elastic events appear primarily in the 
fully damped peak. At an intermediate angle (the grazing angle) the valley between the fully damped and the elastic peak is filled by quasi-elastic events. Even though the energy loss associated with the fully damped component is larger for ${ }^{136} \mathrm{Xe}$ than for ${ }^{84} \mathrm{Kr}$, the damping appears to be more complete for angles near the grazing angle in the case of $136 \mathrm{Xe}$ projectiles. We attribute this to the larger mass and radius of curvature of ${ }^{136} \mathrm{Xe}$, allowing it to "rub" more effectively against the target nucleus than does smaller $84 \mathrm{Kr}$.

The angular distributions for non-elastic scattering are similar to those observed earlier for $\mathrm{Kr}$ on $\mathrm{Pb}$, exhibiting a peak at an angle close to the grazing angle. Integration over the angular distributions for $18 \mathrm{l} \mathrm{Ta}$ and $208 \mathrm{~Pb}$ yield total cross sections comparable to the values of the absorption cross section given by the optical potential obtained in a fit to the elastic scattering angular distribution.

In order to further characterize the deeply inelastic scattering we have used a counter telescope to determine the $z$ distrihutions as a function of energy loss and angle. The telescope consisted of a $10.5 \mu \mathrm{m}$ thick $\Delta E$ detector followed by a surface barrier $E$ detector. The $Z$ resolution (FWHM) at $Z=54$ (Xe) was approximately 2 charge units. This resolution was sufficient to determine the average value and the variance of the charge distribution of the projectile-like products. We give an overview. in Fig. 13.5-5 of the $Z$ distributions at a particular angle in the form of a contour plot of the triple differential cross section as a function of $Z$ and energy loss. The $Z$-distributions for small energy loss (quasi-elastic events). are rather narrow and are centered at the projectile charge. For the deeply inelastic events the distributions become much broader while the average $Z$, remains remarkably unchanged from the $Z$ of the projectile.

We have also calculated the variances of the charge distributions as a function of energy loss and angle. We have plotted both the variance and the square root of the variance, the standard deviation, as a function of energy loss. In the latter case an approximately linear dependence of the standard deviation on energy loss is found which is common to all angles, as illustrated in 


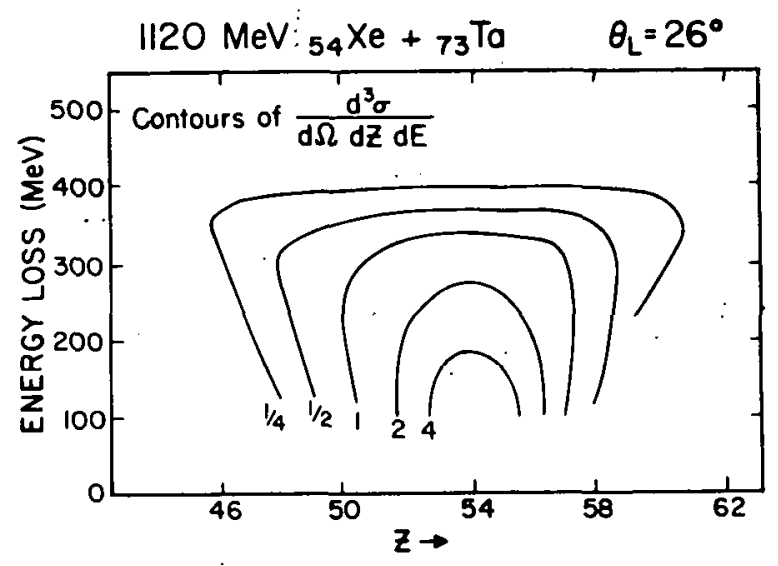

Fig. 13.5-5. Dependence of differential cross section on $Z$ and energy loss at $\theta_{\mathrm{L}}=26^{\circ}$. The cross section contours are in units of $\mathrm{mb} / \mathrm{sr}-\mathrm{MeV}-\mathrm{Z}$.

Fig. 13.5-6. This behavior is qualitatively consistent with diffusion models.

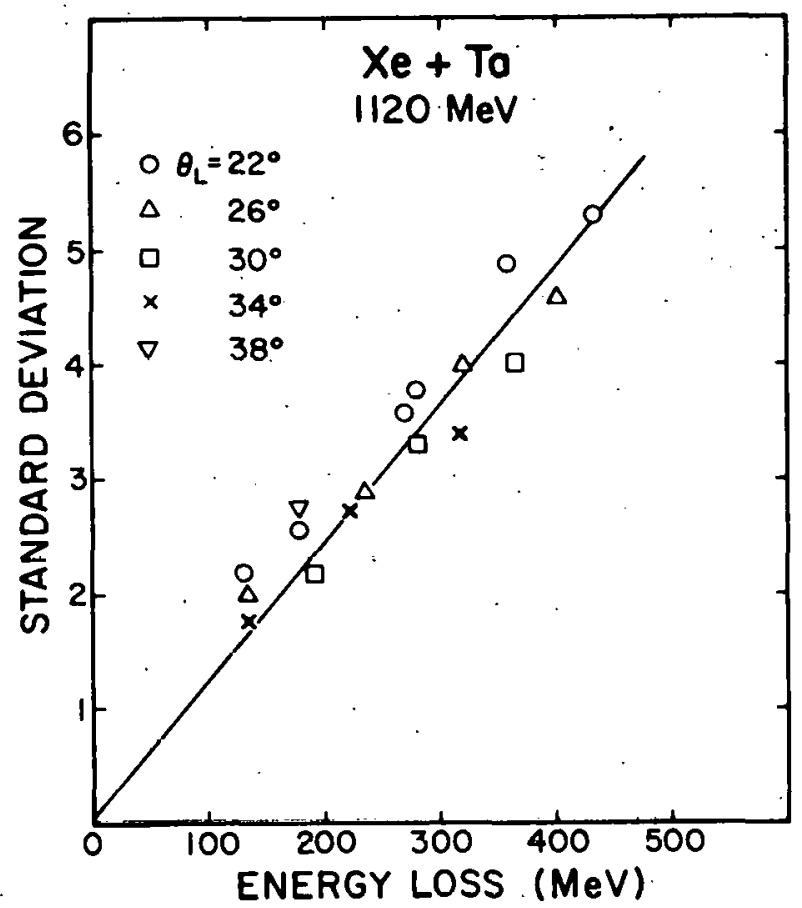

The :model of Nörenberg ${ }^{3}$ predicts that

the variance should depend linearly on

the diffusion time. He assumes that the energy loss varies linearly with time while the nuclei are interacting. This is probably not a good assumption in the present case, as for these strongly damped collisions a maximum energy loss is achieved rather quickly and the rate of energy loss probably slows down as the system evolves toward its guasiequilibrium configuration where most of the available kinetic energy has been converted into internal excitation energy. In this circumstance one would expect the distributions to broaden faster per unit energy loss as the energy loss approaches its maximum value than was predicted by Nörenberg. This is qualitatively consistent with the dependence observed.

+ Permanent address: Oregon State University, Corvallis, OR.

1. R. Vandenbosch, M.P. Webb, and T.D. Thomas, Phys. Rev. Lett. 36,459 (1976).

2. W.U. Schröder, J.R. Birkelund, J.R. Huizenga, K.L. Wolf, J.P. Unik, and V.E. Viola, Jr., Phys. Rev. Lett. 36, 514 (1976).

3. W. Nörenberg, Phys, Lett. $52 B, 289$ (1974). 


\section{HEAVY ION TRANSFER AND INTERMEDIATE STRUCTURE REACTIONS}

14.1 Studies of the Reactions ${ }^{12} \mathrm{C}\left({ }^{14} \mathrm{~N},{ }^{13} \mathrm{~N}\right){ }^{13} \mathrm{C}$ and ${ }^{12} \mathrm{C}\left({ }^{14} \mathrm{~N},{ }^{13} \mathrm{C}\right)^{13} \mathrm{~N}$ at $53.5 \mathrm{MeV}$

R. Bangert, Y-d Chan, J.G. Cramer, B. Cuengco, K-L Liu, W. Lynch, and J. Wiborg

A collaborative investigation of the reaction ${ }^{12} \mathrm{C}\left({ }^{14} \mathrm{~N},{ }^{13} \mathrm{~N}\right){ }^{13} \mathrm{C} *(3.09 \mathrm{MeV})$ at $E=100 \mathrm{MeV}(\mathrm{ab})$ performed at the LBL 88" cyclotron and initiated by members of this laboratory first showed the occurrence of an anomalous angular distribution in a supposedly well-understood heavy ion transfer reaction of a single nucleon with $\ell=1$ uniquely.l Since these results were reported, other groups have observed similar anomalies, often related to $l=1$ single nucleon transfers. ${ }^{2}$

Various explanations of the anomalous angular distributions have been proposed such as core excitation, two-step processes, or configuration mixing, but none has been satisfactory. All the experiments where anomalous effect in the mass 13-14 region appeared were done at high energy ( $100 \mathrm{MeV}$ ). Here we intend to acquire more data in lower energy regions and check. for the persistence of these effects, since energy dependence may provide a clue to their origin.

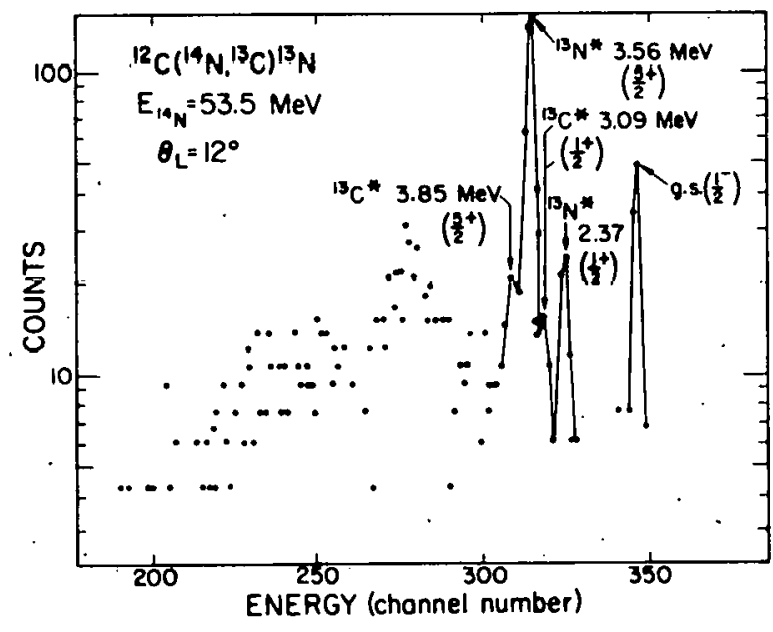

Fig. 14.1-1. Energy spectrum for the $12 \mathrm{C}\left(14_{\mathrm{N}}, 13_{\mathrm{C}}\right)^{13} \mathrm{~N}$ reaction.

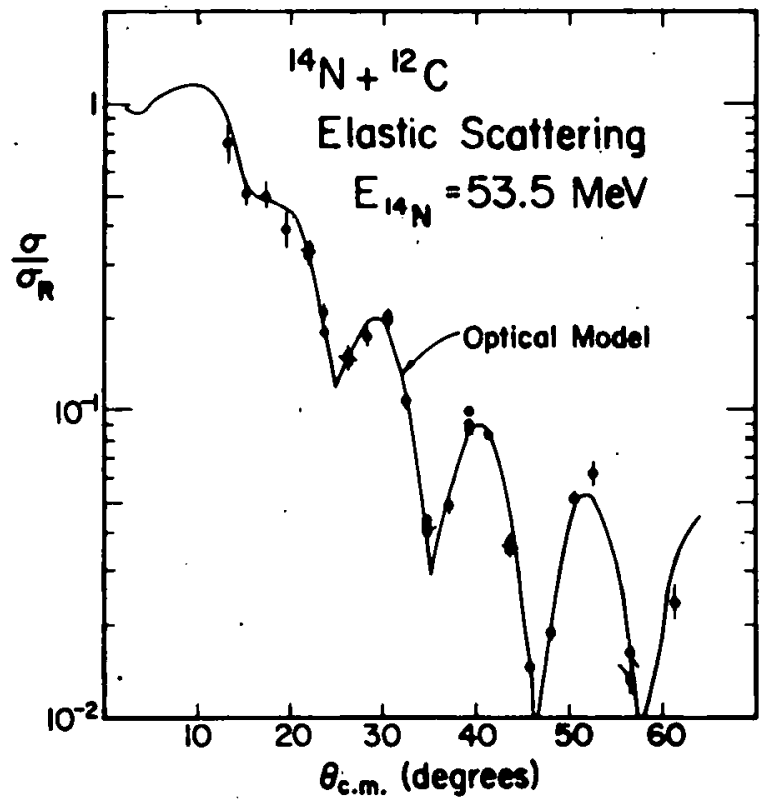

Fig. 14.1-2. Angular distribution for the ${ }^{12} \mathrm{C}\left(1{ }^{14} \mathrm{~N}, 14_{\mathrm{N}}\right)^{12} \mathrm{C}$ reaction. The solid line is an optical potential fitting obtained with the parameter set: $V_{0}=7.09$ $\mathrm{MeV}, \mathrm{r}_{0}=1.44 \mathrm{~F}, \mathrm{a}_{0}=.65 \cdot \mathrm{F}, \mathrm{w}_{\mathrm{vol}}=2.74 \mathrm{MeV}$, $r_{I}=1.51 \mathrm{~F}, a_{I}=.11 \mathrm{~F}$. 
Single nucleon transfer experiment from ${ }^{14} \mathrm{~N}$ to $12 \mathrm{C}$ has been performed at $53.5 \mathrm{MeV}$ and sets of angular distributions have been obtained for the ground states and lower excited states of $13 \mathrm{C}$ and $13 \mathrm{~N}$. The reaction products were detected by a time of flight $\triangle E-E$ telescope as described in Sec. 3.4 of this report. A typical energy spectrum is shown in Fig. 14.1-1. The experimental energy resolution was about $100 \mathrm{keV}$. This resolution has been found to be necessary to separate the $2 S_{1 / 2}$ state of the $A=13$ product from the very strong neighboring $I d_{5 / 2}$ state. With this resolution, peaks coming from the excited states of the detected particles are also observed, as shown in Fig. 14.1-1.

The elastic angular distribution was first measured with the conventional $\triangle E-E$ telescope (Fig. 14.1-2). A large angular $\left(\theta_{\mathrm{c} . \mathrm{m} .}=10^{\circ}\right.$ to $\left.60^{\circ}\right)$ was included to insure a good result in fitting with optical parameters, since difficulty in fitting the elastic scattering was encountered at $100 \mathrm{MeV}$ where only 10 data points were obtained. It was found that with more backward angle data, the optical model parameters were better determined, in that only a shallow real potential produced a good fit to the elastic data. All the potentials deeper than $25 \mathrm{MeV}$ had local minima of larger chi square when fitting the elastic data.

This shallow potential has been used to predict the elastic angular distribution at $100 \mathrm{MeV}$, and a fit has been found which is better than had previously been obtained. This is encouraging since it gives some indication that the average potential is energy independent and can accurately predict elastic scattering over a wide range of energies.

The radial sensitivity of these optical parameters was investigated using the notch perturbation method and the result is shown in Fig. 14.1-3. There is some indication that the sensitivity extends inside the nuclear radius. This could mean that with such a weak potential the interior is contributing.

The transfer reaction data were shown in Fig. 14.1-4, together with the exact finite range DWBA with recoil calculation using the shallow potential obtained from the elastic scattering. For the transfer to the ground state, the deeper potential seems to give a better fit to the forward angle data. However the shallow potential is essential to produce the bump at about $47^{\circ}$. Also 

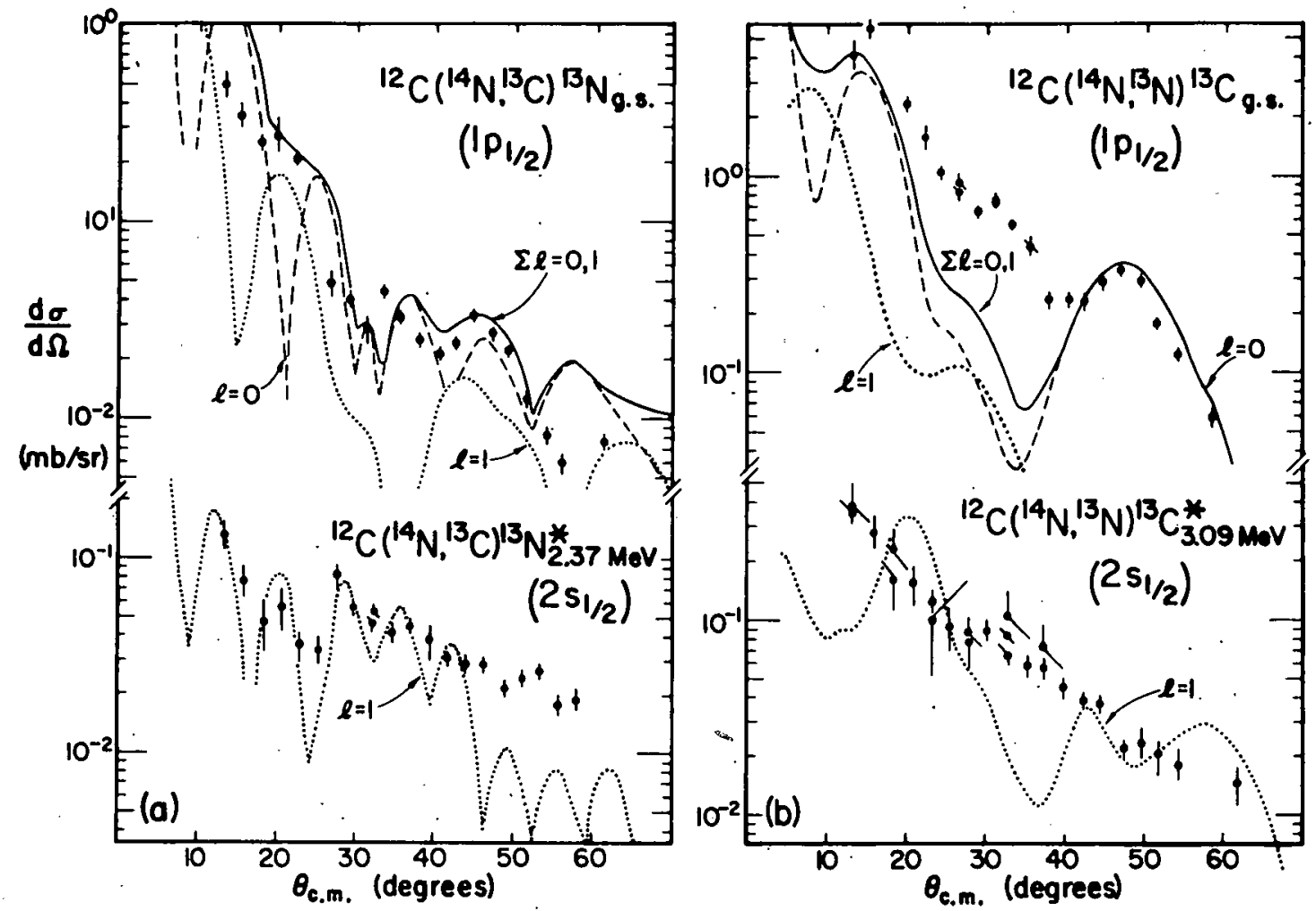

Fig. 14.1-4. Experimentally observed angular distribution for ${ }^{14} \mathrm{~N}+{ }^{12} \mathrm{C}$ transfer reactions at $53.5 \mathrm{MeV}$ and DWBA calculations.

the ohallow potential gives a slope which agrees better with the data.

For the $\ell=1$ transfer to the $2 \mathrm{~S}_{1 / 2}$ state in the ${ }^{12} \mathrm{c}\left({ }^{14} \mathrm{~N},{ }^{13} \mathrm{C}\right){ }^{13} \mathrm{~N}$ reaction, the angular distribution at the forward angle agrees quite well with the calculation, contrasted to the $100 \mathrm{MeV}$ case. This is true for both the cases when the deep and shallow potentials are used, so it is independent of the parameters; but rather an energy dependent effect. However, the mirror transfer reaction to the ${ }^{13} \mathrm{C}\left(2 \mathrm{~S}_{1 / 2}\right)$ state gives nothing to support this argument. This difference between $\mathrm{p}$ and $\mathrm{n}$ transfer and energy dependent effects are currently under study.

1. Nuclear Physics Laboratory Annual Report, University of Washington (1974), p. 103 .

2. K.G. Nair et al., Phys. Rev. C 12, 1575 (1975).

3. See Sec. 12.3 of this report. 
$14.2 \frac{\text { The }{ }^{12} \mathrm{C}+{ }^{16} \mathrm{O} \rightarrow{ }^{8} \mathrm{Be}+{ }^{20} \mathrm{Ne} \text { Reaction }}{{ }^{3}}$

K.G. Bernhardt, H. Bohn, and K.A. Eberhard

One of the most interesting aspects of the nonstatistical intermediate structures above the Coulomb barrier is that they show up only for certain "light" heavy ion entrance channel systems: like $12 \mathrm{C}+12 \mathrm{C}, 12 \mathrm{C}+160$ and $12 \mathrm{C}+18_{0}$ and not for others, and even for a given system only in special exit channels. At present no consistent explanation of these effects is available. One reason for this is the incompleteness of the experimental data.

We decided to extend the information on the ${ }^{12} \mathrm{C}+{ }^{16} 0$ system in two ways: First, by measuring $\gamma$-ray yields from residual nuclei (see Sec. 13.3 of this report) rather than particle yields to specific states, and second by studying less known reaction exit channels. Whereas elasticl and inelastic ${ }^{2}$ scattering $^{1}$ and $n,{ }^{3} \mathrm{p}, 4 \mathrm{~d}, 4$ and ${ }^{4} \mathrm{He}^{4,5}$ reaction exit channels have been studied, little information is available for the 8 Be exit channel. 6,7

We started an intensive investigation of the ${ }^{8} \mathrm{Be}+{ }^{20} \mathrm{Ne}$ exit channel for the $12 \mathrm{C}+160$ system by measuring excitation functions and angular distributions. Here we present some results obtained from the excitation function measurements. Using a 7-detector array we identified the particle unstable ${ }^{8} \mathrm{Be}$ nucleus through the coincident detection of the two $4 \mathrm{He}$-particles by which it decays. Simultaneously the $\alpha+{ }^{24} \mathrm{Mg}$ exit channel was recorded. Tantalum foils were mounted in front of the detectors in order to prevent elastically scattered particles from entering the detector. This technique also allows the measurement of ${ }^{8} \mathrm{Be}$ at ${ }_{\mathrm{CM}}=0^{\circ}$. The excitation functions were measured between $E_{\mathrm{CM}}=13.5 \mathrm{MeV}$ and $23 \mathrm{MeV}$ in $\Delta \mathrm{E}_{\mathrm{CM}}=128 \mathrm{keV}$ steps, covering an angular range in $\Delta \theta_{1 \mathrm{ab}}=5^{\circ}$ steps from $\theta_{1 \mathrm{ab}}=$ $5^{\circ}$ to $35^{\circ}$ for the ${ }^{4} \mathrm{He}$ and $\theta_{\mathrm{lab}}=7.5^{\circ}$ to $32.5^{\circ}$ for the $8 \mathrm{Be}$ exit channel. For the ${ }^{4} \mathrm{He}+{ }^{24} \mathrm{Mg}$ exit channel we analyzed 5 groups of states up to $6.43 \mathrm{MeV}$ excitation energy in ${ }^{24} \mathrm{Mg}$. This channel served for our purposes also as a control reaction for our data because we can compare our results for this channel with a large body of existing data from the literature. ${ }^{5}$

In the ${ }^{12} \mathrm{C}\left({ }^{16} \mathrm{O},{ }^{8} \mathrm{Be}\right){ }^{20} \mathrm{Ne}$ reaction we observe at the higher bombarding energies a strong population of the $\left(0^{+}, \mathrm{g.s.}\right),\left(2^{+}, 1.63 \mathrm{MeV}\right),\left(4^{+}, 4.25 \mathrm{MeV}\right)$ and $\left(6^{+}, 8.75 \mathrm{MeV}\right)$ members of the $\mathrm{g.s}$. rotational band and also of the $\left(1^{-} .5 .80 \mathrm{MeV}\right)$ and $\left(3^{-}, 7.17 \mathrm{MeV}\right)$ members of the excited negative parity band built on the $\left(1^{-}, 5.80 \mathrm{MeV}\right)$ state. We also obtain a weaker indication of the population of $\mathrm{K}^{\pi}=2^{-}$band members. This is in accordance with the $160\left(12 \mathrm{C},{ }^{8} \mathrm{Be}\right) 20 \mathrm{Ne}$ reaction data obtained at $56 \mathrm{MeV}$ bombarding energy. 6 Figure $14.2-1$ displaỹs the analyzed excitation functions for the g.s. in
${ }^{20} \mathrm{Ne}$ and $24 \mathrm{Mg}$ respectively. Figure $14.2-2$ shows the angle integrated cross sections for the g.s. together with the average angle integrated cross sections, obtained with an averaging interval of $\Delta E_{C M}=1.7 \mathrm{MeV}$. A preliminary correlation analys is has been started. Figure 14.2-3 now includes all states up to $7.17 \mathrm{MeV}$ in $20 \mathrm{Ne}$ and $6.43 \mathrm{MeV}$ in ${ }^{24} \mathrm{Mg}$. Displayed is the cross correlation function taken over all states and all angles as defined by L:R. Greenwood et al. 8 The position of the most pronounced nonstatistical intermediate structure for the ${ }^{12} \mathrm{C}+16_{0}$ entrance channel system at $E_{C M}=19.7 \mathrm{MeV}$ is indicated in Figs. 14.2-2 and 14.2-3 

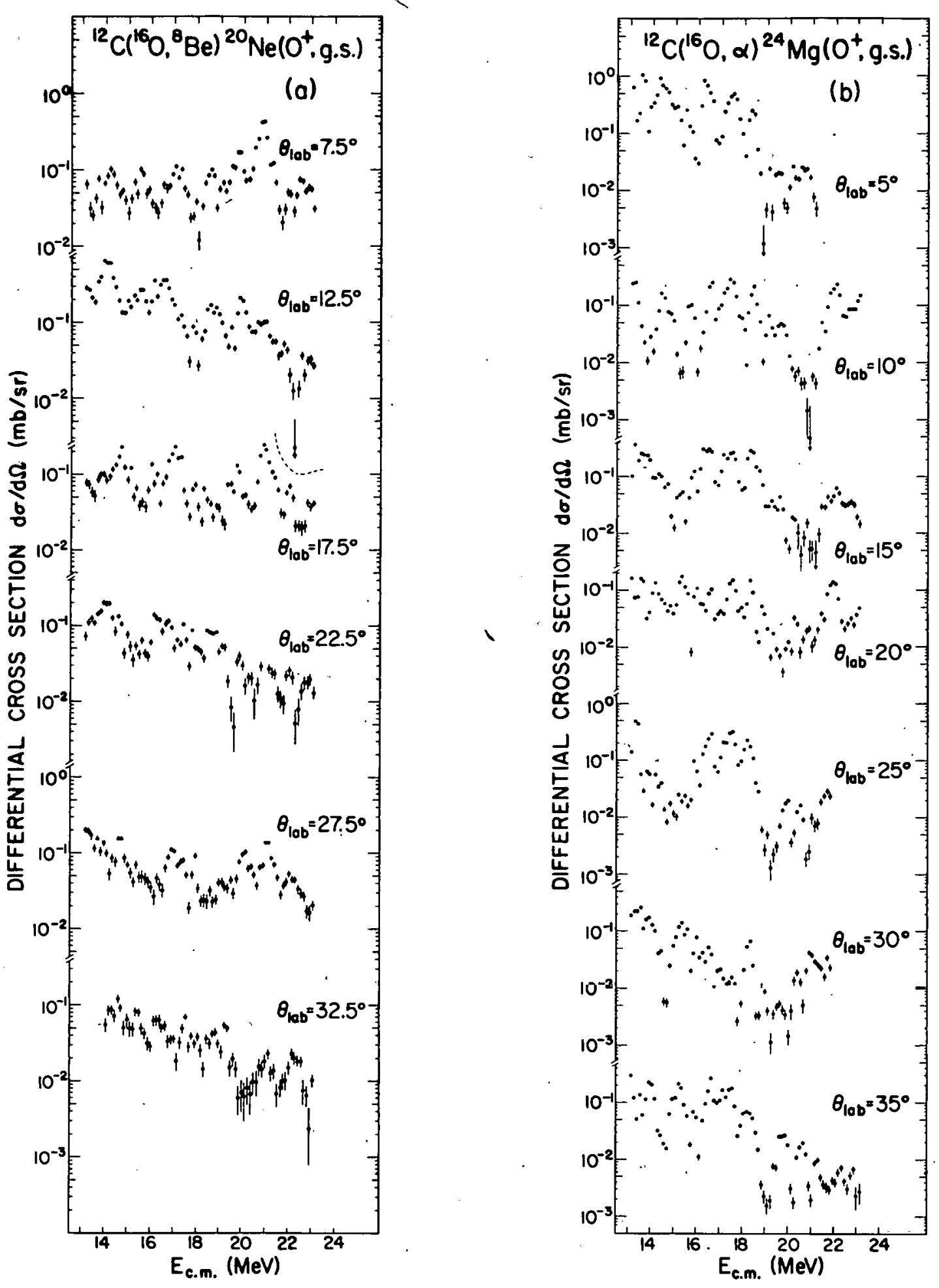

Fig. 14.2-1. Excitation function for the (a) ${ }^{12} \mathrm{C}\left({ }^{16} \mathrm{O},{ }^{8} \mathrm{Be}\right)^{20} \mathrm{Ne}\left(0^{+}, \mathrm{g} . \mathrm{s}.\right)$ reaction; (b) $12 \mathrm{C}\left(160,{ }^{4} \mathrm{He}\right)^{24} \mathrm{Mg}\left(0^{+}, \mathrm{g.s}.\right)$ reaction. 


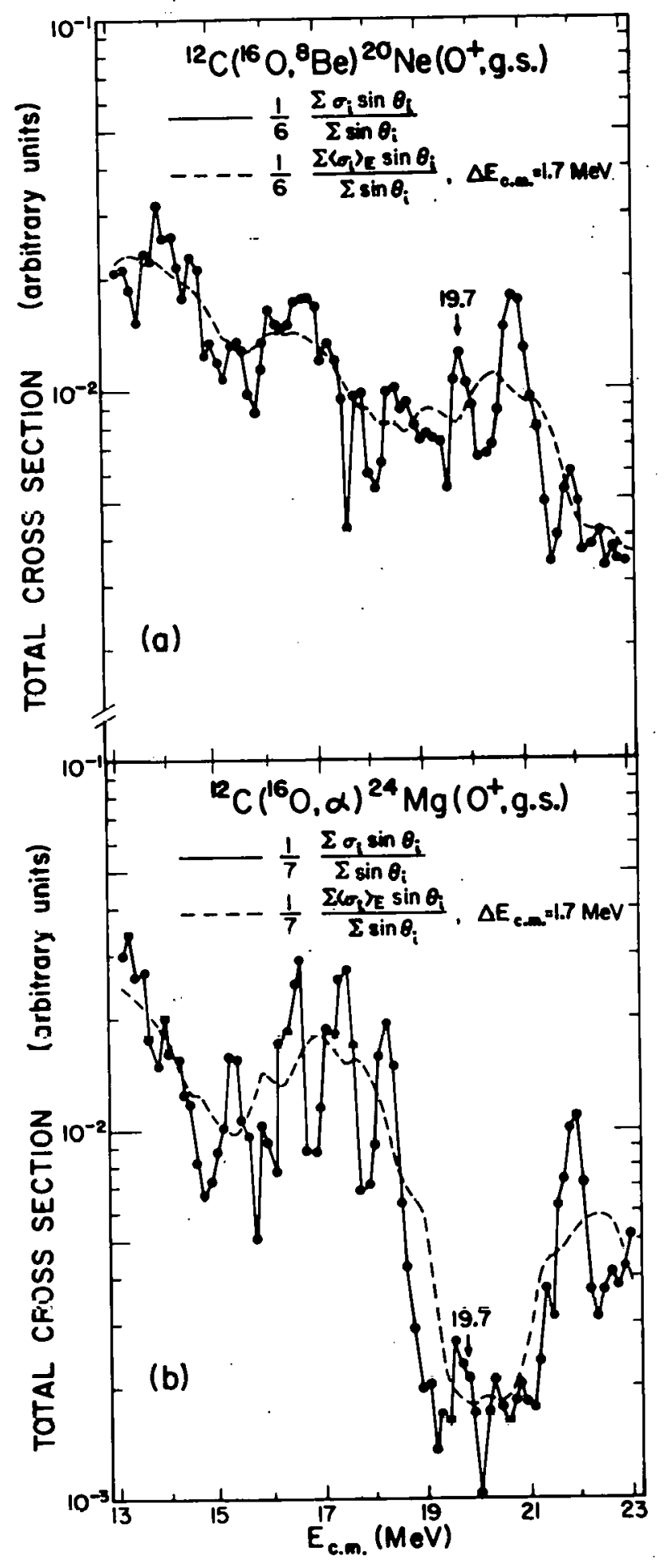

Fig. 14.2-2. Angle integrated and energy averaged excitation function for the (a) ${ }^{12} \mathrm{C}\left(16 \mathrm{O},{ }^{8} \mathrm{Be}\right){ }^{20} \mathrm{Ne}\left(0^{+}\right.$, g.s. $)$reaction; (b) $12 \mathrm{C}\left(16 \mathrm{O},{ }^{4} \mathrm{He}\right)^{24} \mathrm{Mg}\left(\mathrm{O}^{+}, \mathrm{g} . \mathrm{s}\right.$. $)$ reaction.

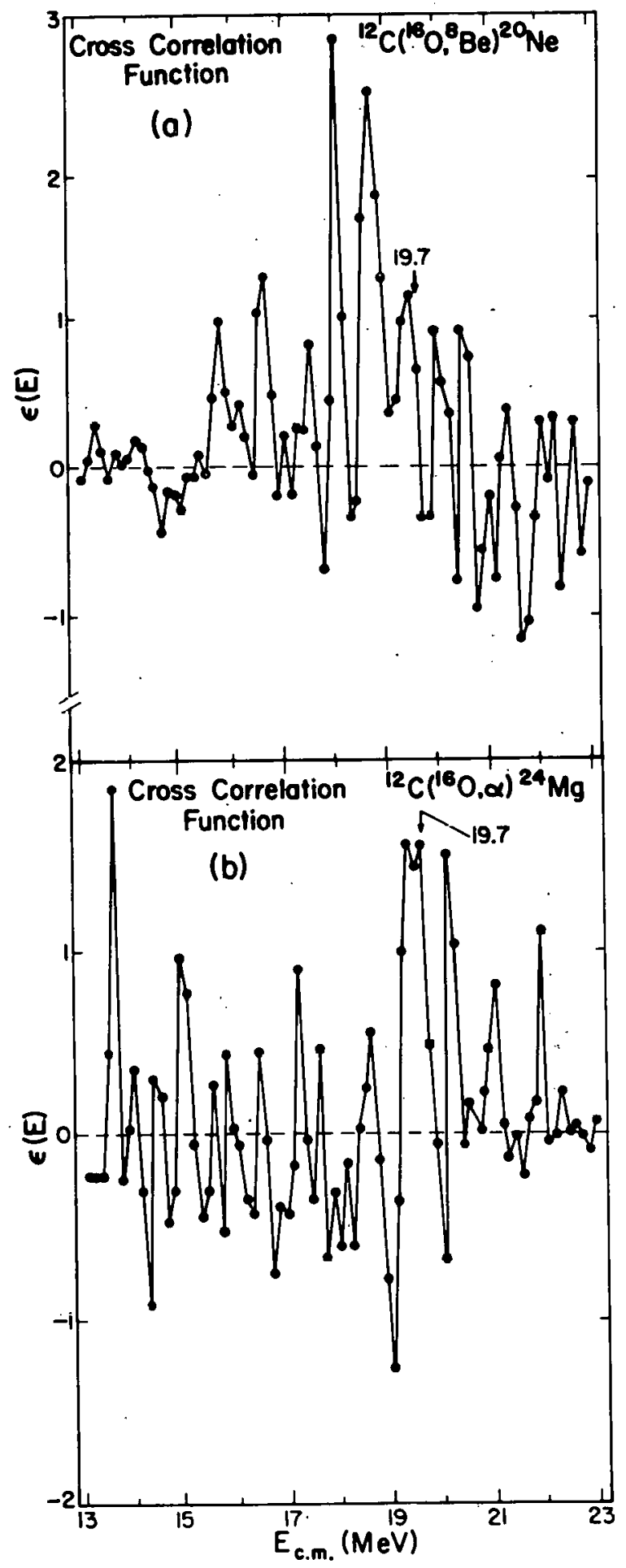

Fig. 14.2-3. State and angle integrated cross correlation function for the

(a) ${ }^{12} \mathrm{C}\left(160,{ }^{8} \mathrm{Be}\right)^{20} \mathrm{Ne}$ reaction;

(b) ${ }^{12} \mathrm{C}\left(160,{ }^{4} \mathrm{He}\right){ }^{24} \mathrm{Mg}$ reaction. 
by an arrow. The enhancement around this energy is evident through all the figures. Besides this our data exhibit strong fluctuations in both exit channels indicative of the importance of compound nuclear processes. This is supported by the fact that Hauser-Feshbach calculations are in agreement with the magnitude of the preliminary experimental cross sections for the g.s. in both exit channels. A more detailed fluctuation analysis together with Hauser-Feshbach calculations is presently underway. The cross correlation function also shows correlated enhancements for both exit channels at other center of mass energies. Detailed angular distribution measurements at those energies are in progress.

Finally we would like to mention that it is also important to get better information about the high spin state population pattern in $20 \mathrm{Ne}$. For example, in the usual $2 \theta / \hbar^{2}$ vs $(\hbar \omega)^{2}$ plot the g.s. rotational band in ${ }^{20} \mathrm{Ne}$ shows a very strong dependence of the moment of inertia $(\theta)$ on the rotational frequency (very similar to backbending plots for rare earth nuclei) with a possible saturation at $\mathrm{J}^{\pi}=8^{+}$. No higher spin states of this band have been established. A similar restriction in angular momentum seems to be true for other bands in $20_{\mathrm{Ne}}$. Recent theoretical calculations try to understand this type of cutoff in terms of self-consistent cranking calculations. 9 It would therefore be interesting to get more detailed experimental data for the higher spin states.

1. R.E. Malmin, R.H. Siemssen, D.A. Sink, and P.P. Singh, Phys. Rev. Lett. 28, 1590 (1972).

2. R.E. Malmin and P. Paul, preprint.

3. P. Sperr, D. Evers, A. Harasim, W. Assmann, P. Konrad, K. Rudolph, G. Denhöfer, and C. Ley, Phys. Lett. 57B, 438 (1975).

4. E.R. Cosman, A. Sperduto, T.M. Cormier, T.N. Chin, H.E. Wegner, M.J. LeVine, and D. Schwalm, Phys. Rev. Lett. 29, 1341 (1972).

5. M.L. Halbert, F.E. Durham, and A. Van Der Woude, Phys. Rev. 162, 899 (1967); and Phys. Rev. 162, 919 (1967); see: Several contributions to "Symposium on Heavy Ton Reartions and Many Particle Excitations", Saclay (1971), published in Journal de Physique C 6(1971); L.R. Greenwood, K. Katori, R.E. Malmin, T.H. Braid, J.C. Stoltzfus, and R.H. Siemssen, Phys. Rev. C 6 , 2112 (1972); J.L.C. Ford, Jr., J. Gomez Del Campo, R.L. Robinson, P.H. Stelson, and S.T. Thornton, Nucl. Phys. A226, 189 (1974).

6. E. Mathiak, K.A. Eberhard, J.G. Cramer, H.H. Rossner, J. Stețtmeier, and A. Weidinger, Nucl. Phys. A2sy, 12y $\left(1 y^{\prime} \%\right)$.

7. D.R. James, G.R. Morgan, N.R. Fletcher, and M.B. Greenfield, Bull. Am. Phys. Soc. II, 20, 1174 (1975)

8. L.R. Greenwood, R.E. Segel, K. Raghunathan, M.A. Lee, H.T. Fortune, and J.R. Erskine, Phys. Rev. C 12, 156 (1975).

9. K.H. Passler and U. Mosel, Nucl. Phys. A257, 242 (1976). 
$14.3 \frac{\text { The }{ }^{12} \mathrm{C}\left({ }^{18} \mathrm{O}, \alpha\right)^{26} \mathrm{Mg} \text { Reaction }}{\mathrm{K}}$

K.G. Bernhardt, 'K.A. Eberhard, R. Vandenbosch, and M.P. Webb

The elastic scattering of ${ }^{18} 0$ from ${ }^{12} \mathrm{C}$ exhibits the novel behavior of being dominated by odd partial waves.l In addition, the elastic scattering excitation functions show the existence of intermediate width structure ( $\Gamma$ r $600 \mathrm{keV}(\mathrm{c.m})$.$) correlated in angle. These$ structures appear at 16.6, 19.0 and 21.8 $\mathrm{MeV}(\mathrm{c} . \mathrm{m}$.$) . Angular distributions sug-$ gest that the 19.0 and $21.8 \mathrm{MeV}$ "resonances" are dominated hy the $\ell=15$ and $\ell=17$ partial waves, respectively. We have studied the $\alpha+26 \mathrm{Mg}$ reaction channel to see if such structures appear in this channel at the same energies.

${ }^{18} 0$ beams were used to bombard 50-100 $\mu \mathrm{g} / \mathrm{cm}^{2}{ }^{12} \mathrm{C}$ targets. The $\alpha$ spectra were obtained with a transmission mounted, surface barrier $\Delta \mathrm{E}$ counter $(250 \mu$ thick) backed by a veto counter to suppress contributions from protons and deuterons. Excitation functions at $153^{\circ}$ and $169^{\circ}$ (c.m.) were measured over an energy range from 15.6 to $22.4 \mathrm{MeV}$ (c.m.). Reactions leading to twelve states in $26 \mathrm{Mg}$ could be identified and all exhibited rapidly fluctuating cross sections. Figure 14.3-1 shows the deviation function calculated for $\theta_{\mathrm{c} . \mathrm{m}}$. $=169^{\circ}$ ( Fig. 14.3-1b) along with that calculated for the elastic scattering (Fig. 14,3-la). Correlations appear in the $\alpha+{ }^{26} \mathrm{Mg}$ channel at 16.6, 19.0 and $21.8 \mathrm{MeV}$. An angular distribution at $19.0 \mathrm{MeV}$ was measured for the $\alpha+{ }^{26} \mathrm{Mg}$ (g.s.) channel and the results are shown in Fig. 14.3-2. 'The periodicity of the data is constant with a $\left|P_{15}\right|^{2}$ behavion over an angular range of $50-100^{\circ}$. Between $100^{\circ}$ and $120^{\circ}$ however there is evidence for the contribution of more than a single partial wave.

The cross correlation in energy of the intermediate width structures is consistent with the behavior expected for the so-called "quasi-molecular" resonances. However, it would appear that the suggested condition that the entrance

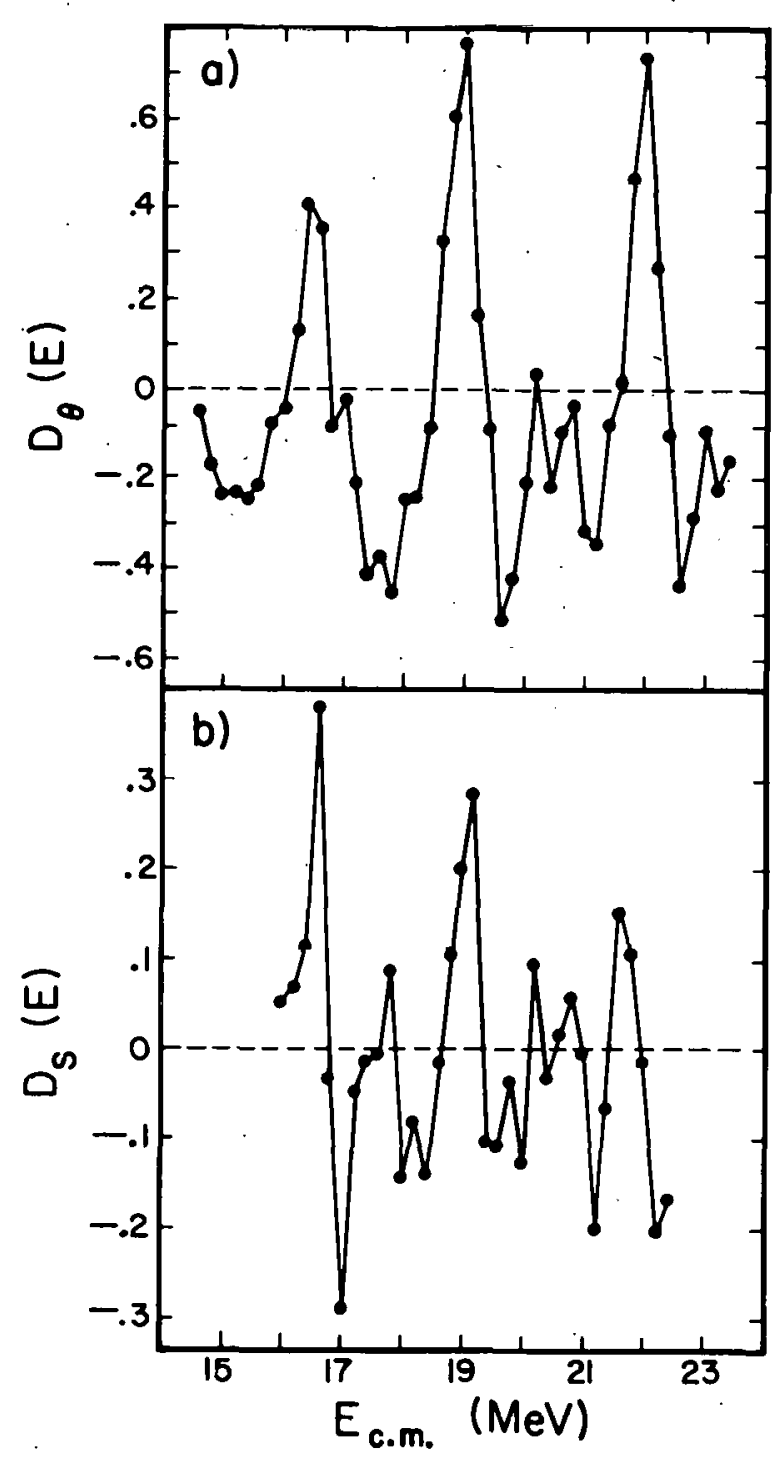

Fig. 14.3-1. The deviation function for a) the elastic scattering at eight angles, $D_{\theta}(E)=(1 / 8) \sum\left[\left(\sigma_{i}-\left\langle\sigma_{i}\right\rangle\right) /\left\langle\sigma_{i}\right\rangle\right]$ and $\left.b\right)$ for the $12 \mathrm{C}\left(18_{0}, \alpha\right)^{26} \mathrm{Mg}$ reaction for twelve. states in $26_{\mathrm{Mg}}$ at $\theta_{\mathrm{CM}}=169^{\circ}, \mathrm{D}_{\mathrm{S}}(\mathrm{E})={ }^{\circ}$ $(1 / 12) \Sigma\left[\left(\sigma_{i}-\left\langle\sigma_{i}\right\rangle\right) /\left\langle\sigma_{i}\right\rangle\right]$. The averaging interval was $1.0 \mathrm{MeV}(\mathrm{c} . \mathrm{m}$.$) in both cases.$ 


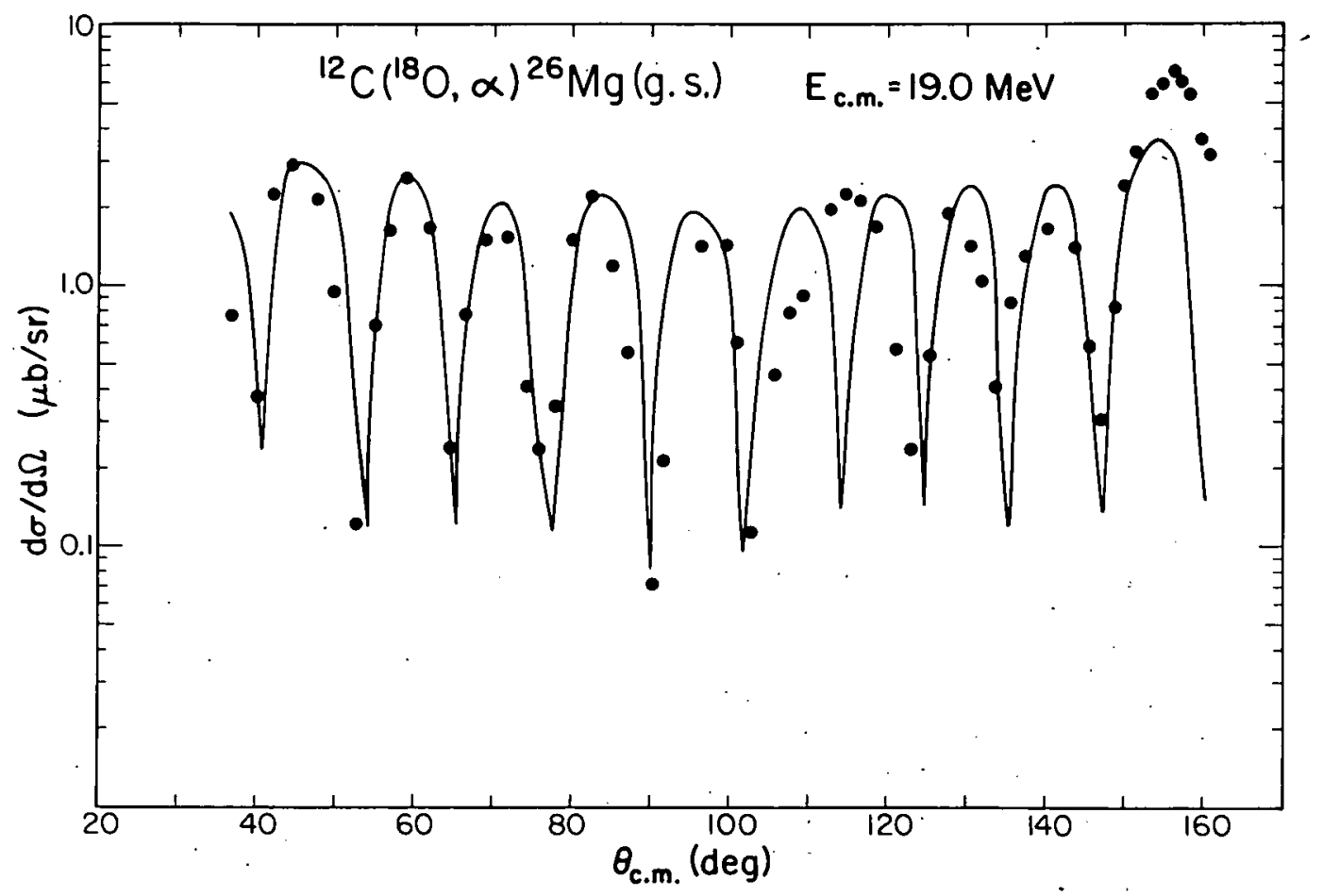

Fig. 14.3-2. ${ }^{12} \mathrm{C}\left({ }^{18} \mathrm{O}, \alpha\right){ }^{26} \mathrm{Mg}(\mathrm{g.s.}$ ) $)$ angular distributions at $\mathrm{E}_{\mathrm{CM}}=19.0 \mathrm{MeV}$. The solid curve is an arbitrarily normalized $\left|P_{15}\right|^{2}$.

channel must consist of $\alpha$-particle nuclei and that the compound nucleus level density need be low for the observation of such resonances ${ }^{2}$ does not hold in the 180 i. $12 \mathrm{C}$ ayctem.

1. M.P. Webb, R. Vandenbosch, K.A. Eberhard, K.G. Bernhardt, and M.S. Zisman, Phys. Rev. Lett. 36, 779 (1976).

2. H. Voit, P. Duck, W. Galster, E. Haindl, G. Hartmann, H-D. Helb, F. Siller, and G. Ischenko, Phys. Rev. C10, 1331 (1974). 


\title{
15. MEDIUM ENERGY PHYSICS \\ 15.1 Total Pion Cross-Sections
}

\author{
I. Halpern, L. Knutson and Collaborators ${ }^{\dagger}$
}

We have been waiting impatiently for the end of the Great Shutdown at LAMPF, since our second and final run on total pion cross-sections was scheduled as the first post-shutdown experiment on the Low Energy Pion Channel. The Shutdown is finally over and we are, in fact, running at this very moment. During the 2 year gap since our first run, we have made some important changes in the experimental apparatus and they are described below (see the 1975 Annual Report, p. 201, for a rough description of the basic arrangement of detectors).

The measurement of a total cross-section is neither a measurement of the geometric structure of the target nucleus, nor of the geometrical features of the projectile-nucleus interaction. It is unfortunately a mixture of the two. In The current run we hope to separate these features somewhat. In the neighborhood of the $(3,3)$ resonance where pions are strongly absorbed, we expect that our measurements will be mainly sensitive to nuclear size parameters, but at much lower incident energies where nuclei are rather transparent to pions, we would like to learn some new things about the pion-nucleus interaction.

As regards the nuclear structure studies near the resonance, we had long ago decided to concentrate on isotope comparisons rather than on determinations of very precise values for effective radii of particular nuclei. For one, such "precise" determinations are made somewhat uncertain by the model-dependent corrections one is required to make to the data. Moreover there are equivalent uncertainties in the theoretically expected values of nuclear radii. In short, it is questionable whether one can arrange useful confrontations between theoretical and experimental absolute radius values. However both these types of uncertainties are largely eliminated if one compares cross-sections for different isotopes. say $40 \mathrm{Ca}$ and $40 \mathrm{Ca}$ since the differences in some of the critical uncentainties between the isotopes may be expected to be small both in theory and experiment. For example, although it may be difficult on the basis of our measurements of $48 \mathrm{Ca}$ to be very definite about their implications for the difference between neutron and proton rms radii in this nucleus, it is likely that we will be in a gond position to be able to say: "If the ${ }^{40} \mathrm{Ca}$ results are interpreted with the assumption that the neutron and proton rms radii in this nucleus are equal, then it follows that the difference between these radii in $48 \mathrm{Ca}$ has the value..." That is, we should be in a relatively strong pnsition to compare measurcd iootope differences to theoretically calculated differences.

This emphasis on isotope differences exploits the large pion intensities available at LAMPF since higher intensities allow the accumulation of adequate statistics with thinner targets. Our targets are an order of magnitude thinner than those of earlier measurements ${ }^{l}$ and are, in fact, in the range $\left(\sim \mathrm{gm} / \mathrm{cm}^{2}\right)$ where it just becomes feasible to use enriched isotopic targets. There are moreover additional advantages in using thinner targets; for example smaller effects of secondary interactions and less uncertain corrections for effects of $\pi-\mu$ decay. 
Another possible reason that measured isotope differences of total crosssections can be more meaningful than the absolute values of these cross-sections has to do with the extrapolation procedures used to obtain the cross-sections. In measuring so-called total cross-sections for charged projectiles, it is necessary to extrapolate inward from large angles to eliminate the contributions due to coulomb scattering. It is probably better to extrapolate the measured ratios of the partial cross-sections for, say, ${ }^{48} \mathrm{Ca}$ and $40 \mathrm{Ca}$ rather than to extrapolate the individual cross-sections if the main object is to determine the extrapolated value of the ratio. This is because the angular dependence of the ratio is simpler than that of the cross-section. The accuracy of the final results in total cross-section experiments depends sensitively on the extrapolation procedures and we have been concerned about them. One member of the group has developed a method ${ }^{2}$ for the extrapolation of such cross-sections that appears to improve the precision of heavy element cross-section determinations substantially.

Very recently there has been considerable progress in the determination of rms radii in nuclei both in theory and experiment. In particular there have been some careful comparisons of neutron and proton rms radii, $r_{n}$ and $r_{p}$, in the calcium and. lead isotopes. ${ }^{3-7}$ By and large, the measurements (and the theory) show that $r_{n}-r_{p}$ is less than one tenth fermi in lighter elements although it may be slightly larger for heavy elements. There are however remaining disagreements, some of which may come from the difficulty of distinguishing the geometry of interaction from that of nucleon distribution. It is hoped that our calcium measurements will make a useful contribution to this problem. One advantage that pions have over other projectiles for radius determinations is that they come with both charges, + and -. The demand for consistency between cross-sections determined with pions of both signs can be a most helpful constraint. As an additional constraint, we plan to measure cross-section differences for pairs of isotones $(\mathrm{N}=20,24,28)$ in the calcium region.

Preliminary calculations with available optical models by a member of the group show that our expected statistical accuracies ( $1 \%$ ) should easily suffice to assign a value to $r_{n}-r_{p}$ for ${ }^{48} \mathrm{Ca}$ if, as we have indicated, one assumes a value for this difference in ${ }^{40} \mathrm{Ca}$.

Figure 15.1-1 shows a plot of the calculated differences of $\pi^{ \pm}$total cross-sections in ${ }^{48} \mathrm{Ca}$ and ${ }^{40} \mathrm{Ca}$ for different assumed values of $r_{n}-r_{p}$ for ${ }^{48} \mathrm{Ca}$. In these plots it is also assumed that $r_{n}(40 \mathrm{Ca})=r_{p}(40 \mathrm{Ca})=r_{p}(48 \mathrm{Ca})$. Although the curves in Fig. 15.1-1 were determined using a Kisslinger potential, we find that other reasonable potentials give very similar results. It is not yet clear to us which optical models are best for determining relative $r_{n}$ 's and $r_{p}$ 's or for that matter, whether the rms radii for neutrons and protons are the most appropriate parameters to describe the results of pion total cross-section measurements. We hope to improve our understanding of these matters in the near future. following:

The major changes in our experimental setup since the last run are the

(1) The construction of a rapid-cycling target changer to ensure that targets which are being compared (including blank or background targets) see 
equivalent pion beams.

(2) The fabrication of new target holders to reduce the amount of extraneous mass in the neighborhood of the targets. It is never clear exactly how to construct an appropriate blank for any given target. We have therefore constructed a number of carbon targets of different areas, thicknesses, etc., each with its own blank. We were gratified to learn at the beginning of this run that within high precision they all. give identical values for the crosssection.

(3) A multiple-event rejection counter to eliminate troublesome accidental events that arise when an incident pion follows too close upon the heels of another.

(4) The construction of a new stack of transmission counters using scintillator material half as thick as that in our original stack. This is to permit us to measure cross-sections for low energy ( $250 \mathrm{MeV})$ pions. As we have indicated earlier, although isotope comparison is receiving the main emphasis in this run we will also explore the possibility of measuring total crosssections at lower pion energies than have so far been looked at.

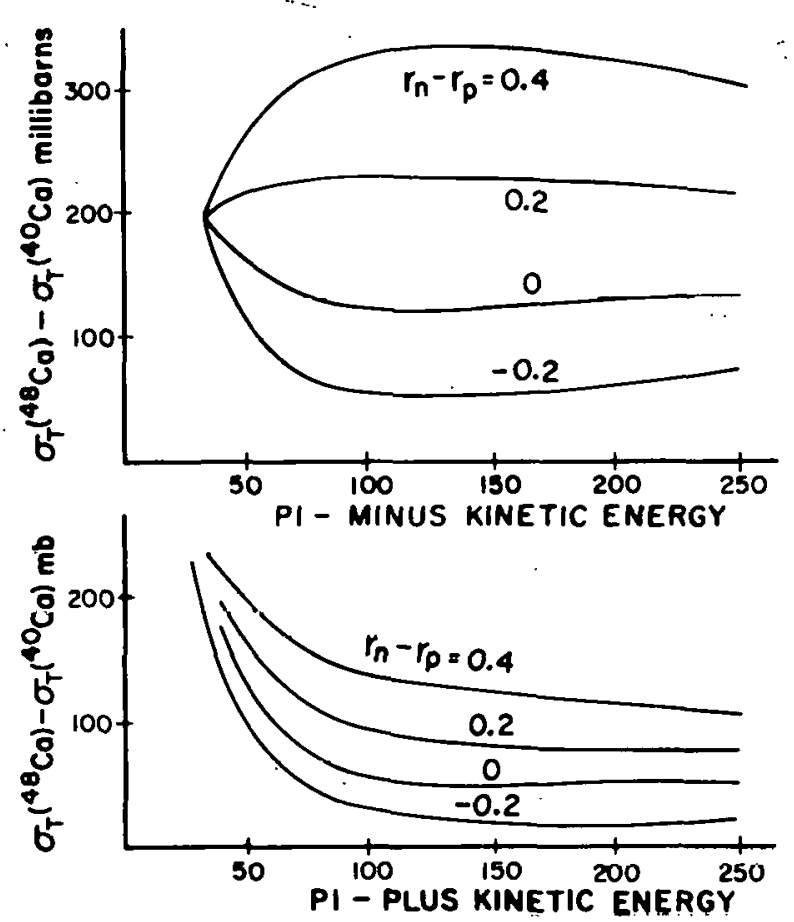

Fig. 15.1-1. Calculated cross-section differences of pions on ${ }^{48} \mathrm{Ca}$ and ${ }^{40} \mathrm{Ca}$. In the region of the $(3,3)$ resonance, a $0.1 F$ change in the value of the rins radius difference $r_{n}-r_{p}$ in $48 \mathrm{Ca}$ results in a $40 \mathrm{mb}(24 \%)$ change in its total croso...ection for negative pions. The corresponding change for positive pions is $\sim 16 \mathrm{mb}$.

(5) Improvement of the data acquisition program to permit the on-line monitoring of critical parameters.

Finally it should be mentioned that we will be collaborating, immediately after our run, with Drs. H. Marshak and T.R. Fisher (of NBS and Lockheed respectively) on total pion cross-section measurements of holmium. We will be using their cryogenic holmium target (which can provide both aligned or unaligned holmium nuclei) as well as our total cross-section apparatus. The general object of these rather forward-looking measurements is to extend the pion techniques to measurements of higher moments of the nucleon distributions than mean radii of the kind we have considered above. Effects due to differences in quadrupole deformation parameters of neutrons vs protons are expected to be rather small, 8,9 but may be just measurable, especially if one complements the total cross-section information with some of the rudimentary angular distribution information available in these experiments. 
$+\quad$ Our collaborators:

University of Montana (M. Jakobson, R. Jeppesen)

Los Alamos Scientific Laboratory (M. Cooper, D. Hagerman, R. Redwine)

Stanford University ( $\mathrm{J}$. Calarco)

California Institute of Technology ( $R$. Marrs)

University of Basel ( $H$. Meyer)

New Mexico State University (G. Burleson, K. Johnson)

1. B.W. Allardyce et al., Nucl. Phys. A209, I (1973).

2. M.D. Cooper and M. Johnson, Nucl. Phys. (to be published).

3. J.C. Lombardi, R.N. Boyd, R. Arking and A.B. Robbins, Nucl. Phys. A188, 103 (1972).

4. M.C. Bertin et al., Nucl. Phys. A167, 216 (197! ).

5. J.W. Negele, Phys. Rev. C 1, 1260 (1970).

6. G.M. Lerner et al., Phys. Rev. C 12, 778 (1975).

7. G.D. Alkhazov et al., Phys. Lett. 57B, 47 (1975).

8. M.S. Iverson and E. Rost, Phys. Rev. C 12, 1589 (1975).

9. M. Jacob and O. Kofoed-Hansen, Nucl. Phys. B17, 565 (1970).

15.2 Ratios of ${ }^{11} \mathrm{C}$ Activations Induced by Pions on Various Targets

E.D. Arthur ${ }^{\dagger}$, D. Chiang and I. Halpern

All light element targets show a conspicuous peak in the total crosssection for pions at the energy of the $(3,3)$ pion-nucleon resonance. 1 In planning our own total cross-section measurements (see Sec. 15.1) we realized that it would be helpful for understanding the pion-nucleus interaction to see just how this resonance changed from target to target. It was also realized that the changes in total cross-sections are likely to be less interesting than those in partial cross-sections since total cross-sections correspond to the incoherent addition of cross-sections for many unrelated processes. It was decided to study the cross-sections for ${ }^{1 I_{C}}$ production from $1 l_{B},{ }^{\prime 2} \mathrm{C}$ and ${ }^{13} \mathrm{C}$ as a function of pion energy by counting the induced $20.3 \mathrm{~min} l l_{C}$ activities.

These reactions have all been studied before. ${ }^{2-4}$ The point of the present investigation is to measure the ratios of cross-sections of these various reactions simultaneously by exposing the targets to the same pion beam and by councing the induced activities in a cycled manner in a controlled geometry. One would hope, in this way, to reduce relative uncertainties in cross-section ratios to around $2 \%$ at energies across the $(3,3)$ resonance. This would constitute a substantial improvement over comparisons of the presently available cross-sections. It would put one in a position (1) to detect any intermediate structure in the $(3,3)$ resonance in nuclei. (The measured cross-section ratios would fluctuate with changing energy instead of lying on a smooth curve) and (2) to detect slight changes in shape or in position of the $(3,3)$ resonance in different nuclei. (Note, for example, that if a resonance is displaced by only $1 \%$ of its width, the ratio of cross-section ratios at the two half-peak points is changed by $\sim 10 \%$.)

A counting system has been assembled and tested that permits precise comparison of annihilation radiation coincidences from positrons emitted by a pair of sources. Since this type of measurement needs only short widely-spaced 
exposure times it makes sense to run it interleaved with another experiment rather than to run it on its own. We have arranged to do these measurements along with our total cross-section measurements. If the results seem interesting we would plan to extend the measurements to additional targets and activities.

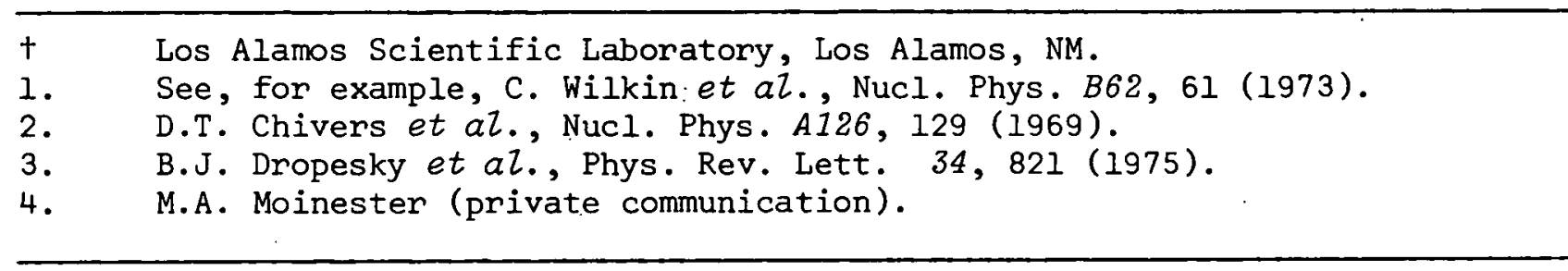

\subsection{Excitation of Giant Resonances by Inelastic Pion Scattering}

n. Chiang, M. Conpen ${ }^{\dagger}$, I. Halpem and L. Knutcon

In last year's Annual Report ${ }^{1}$ we reported in detail our successful feasibility study on the use of intrinsic. Ge detectors to measure broad range inelastic pion spectra. We also explained why pions, especially low energy ( $\sim 50 \mathrm{MeV}$ ) pions can be expected to be more informative than the previously used strong-interaction projectiles. On the basis of our findings additional beam time was applied for and was granted. We-are scheduled to run these measurements in the fall. During the last year or so the problem of higher-lying giant resonances has become even more interesting and puzzling than one at first supposed. It appears that the E2 isoscalar and isovector resonances in light nuclei are broadly dispersed in excitation energy whereas in heavy nuclei they seem to be about as concentrated in energy as the well-known giant dipole resonance. This is not understood. We are in the process of choosing targets and calculating expected results in order to be able to best use the allotted machine time.

$+\quad$ Los Alamos Scientific Laboratory, Los Alamos, NM. Our other colllaborators on this problem are the members of the Carnegie-Mellon medium energy group.

1. Nuclear Physics Laboratory Annual Report, University of Washington (1975), p. 204 . 


\section{ENERGY STUDIES}

\subsection{Energy Studies}

\section{Bodansky and F.H. Schmidt}

The energy study program, begun in early 1973, continued during the past year. Our objectives remain the same as indicated last year; ${ }^{1}$ viz., to understand as best as possible the energy problem as a whole.'

In early 1974 we completed a first draft of a laboratory report on energy. issues. It elicited considerable comment from a wide spectrum of readers, many of whom offered valuable suggestions.

A second draft entitled The Energy Controversy: The Role of Nuclear Power, was completed in August 1975, and some 400 copies were distributed, mostly to persons and organizations who had heard about the first draft and desired copies.

Subsequently we set about to augment the original manuscript with extensive additional material, especially dealing with the waste disposal problem, breeder reactors, plutonium hazards, energy resources, and light water reactor safety. The resulting manuscript is being published in book form (Albion Publishing Co., San Francisco, CA) under the title "The Energy Controversy: The Fight over Nuclear Power", with publication expected to be late summer, 1976.

As the debate over nuclear power intensified, our activities have been extended to the public domain in the form of seminars, debates, and talks to governmental, civic, and other groups.

A corollary, or spin-off, resulting from the distribution of copies of our second draft was an invitation by The American Assembly, Columbia University, to write a chapter on nuclear safety issues in a book to be published by that organization, under the title "The Nuclear Power Controversy". We also participated in a four-day symposium at Arden House; sponsored by The American Assembly, for which the book manuscript served as the source material.

We expect to complete all tinal details of "The Energy Controversy: The Fight over Nuclear Power" by the end of May 1976. Beyond that, the future activities of the energy studies "group" are uncertain, but it is unlikely that we will be able to drop the issues entirely.

1. Nuclear Physics Laboratory Annual Report, University of Washington (1975), p. 207. 


\section{RESEARCH BY USERS AND VISITORS}

17.1 Fast Neutron Beam Radiotherapy -- Medical Radiation Physics*

H. Bichsel $^{\dagger}, \mathrm{J}$. Eenmaa $^{\dagger}, \mathrm{R}$. Seymour $^{\dagger}$, K. Weaver $^{\dagger}$, and P. Wootton ${ }^{\dagger}$

The activities of the Medical Radiation Physics Division during the past year at the Nuclear Physics Laboratory can be grouped into five categories:.

a) measurement and analysis of basic dosimetry data,

b) design and installation of new equipment and systems,

c) repair and maintenance of existing equipment,

d) support of routine therapy operations, and

e) support of other users.

Progress in dosimetry investigations and new equipment design and installation has been significant and will be described in greater detail.

Measurement and analysis of basic dosimetry data. During the past year, measurements of depth dose curves and transverse dose profiles were made in a standard tissue equivalent phantom for square and circular fields. The phantom was a $30 \mathrm{~cm}$ Lucite cube filled with fluid of density $1.07 \mathrm{~g} / \mathrm{cm}^{3}$. Similar data were also measured using square fields with smaller and larger phantoms, and fluids of densities $1.01 \mathrm{~g} / \mathrm{cm}^{3}$ and $1.11 \mathrm{~g} / \mathrm{cm}^{3}$. The results of these measurements were reported at the San Antonio meeting of the American Association of Physicists in Medicinel ( 3 August 1975). From the data measured with the standard phantom, backscatter factors and tissue-air ratios were derived. These data were used to prepare dosimetry calibration charts used for therapy related dose calculations. Dose buildup curves, the effects of field shaping wedges, shlelding effectlveness, background radiation, and fast and slow neutron fluences in the therapy beam were also measured.

Neutron radiotherapy beams are unavoidably contaminated by photons. Since the photons have a significantly lower biological effectiveness than neutrons, the fraction of the total dose that is due to photons should be known. The photons dose fraction in the neutron beam was measured as a function of field size, phantom size, depth in phantom, and transverse displacement using two techniques: the paired chamber technique and the tissue equivalent proportional counter technique. Agreement between resuits of the two methods was comparable to the overall experimental uncertainty. Experimental results were presented at the San Antonio AAPM meeting mentioned above, 2 and are available as a publication in preprint form.

Measurements using chemical dosimeters such as ferrous sulfate and cer1c sulfate solutions have been made. No results are available yet, and this work is continuing. 
Design and installation of new equipment and systems. The therapy facility has been improved by the design or provision of several new devices and capabilities. The most notable is a new, custom designed therapy chair. This chair is suspended from the ceiling of the therapy room and has motorized variablespeed drives on five axes (rotational, vertical, longitudinal, lateral, and $t i l t$ ). The chair converts to a reclining configuration, and can be replaced on its mount by a bed or a standing/kneeling support. Work is under way to provide computer control of the chair position and orientation.

Other new devices include magnetically held steel blocks for external field shaping, a port film holder for neutron radiographs, and a high pressure gas target designed for preliminary dosimetry studies. (Calculations show that the average energy of neutrons in the therapy beam could be increased by about $50 \%$ by use of a $\mathrm{D}_{2}$ gas target (pressurized to $40 \mathrm{~atm}$ ).

New uses of the Raytheon 704 computer have been implemented. These include development of a therapy interactive program and a program for kerma calculations. The therapy program allows storage and retrieval of dosage data for all therapy patients, calculates dial settings for the therapy controller that result in prescribed dosages for a variety of treatment configurations, calculates skin doses, monitors the therapy control electronics, and automatically records dose delivered, time, date and electronics settings for each treatment. The kerma program has been used to calculate neutron kerma/fluence values for $A l$ and $S$ for use in paired-chamber and chemical dosimetry measurements.

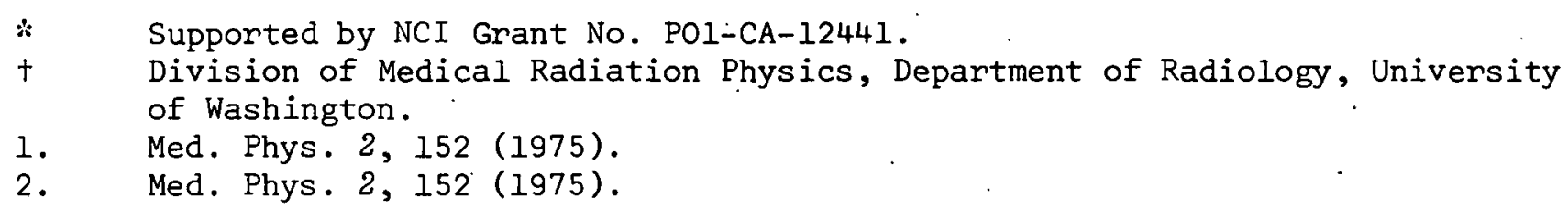

17.2 Fast Neutron Beam Rad1otherapy -- Experimental Oncology\%

J.S.R. Nelson ${ }^{\dagger}$

The research program in experimental oncology is supportive of the neutron beam therapy project which is currently treating cancer patients with the neutron beam from the University of Washington cyclotron. Our program attempts to answer clinically relevant questions in the neutron radiobiology of tumors. following:

Projects completed, continued or initiated in the past year include the

a. Preoperative irradiation of C3HBA mammary adenocarcinomas of $\mathrm{C} 3 \mathrm{H}$ mice with $\mathrm{X}$-rays and neutrons has been nearly completed. (These studies were done in part by an M.S. candidate in Radiological Sciences, Tore Straume.)

СзнBA tumors growing on the hind legs of $\mathrm{c} 3 \mathrm{H} / \mathrm{HeJ}$ mice were irradiated with single fractions of X-rays or neutrons 48 hours prior to surgical excision. Animals treated by surgery alone had a $34 \%$ recurrence rate. A dose of 350 rads of neutrons or 1160 rads of X-rays reduced primary tumor recurrence to $17 \%$ ( $50 \%$ 
of control value), yielding a neutron RBE of 3.3. This is lower than the RBE of 3.7 for growth delay at the same X-ray dose. Mice whose tumors were irradiated with neutrons received a whole body dose equal to $5-7 \%$ of the tumor dose; this appeared to increase the incidence of lung metastases in mice who received high preoperative doses and whose tumors did not recur. X-irradiated animals received a negligible whole body dose and did not have lung metastases when the primary had not recurred. This problem was further studied by irradiating directly the lungs of animals whose tumors were treated by preoperative radiation plus surgery. Both $\mathrm{X}$-rays and neutrons given directly to the lungs increased the number of lung metastases in mice with primary tumors transplanted on the leg and preoperatively irradiated with 300 rads neutrons or 500 rads $X$-rays. A saturation effect appeared to be reached at a dose of 50-75 rads neutrons or 150 rads $X$-rays to the lungs.

b. The respons of the EMT-6 solid tumors growing in BALB/c mice to single doses of $X$-rays or cyclotron produced neutrons is being studied, comparing several endpoints of tumor response.

Prior to studying EMT-6 tumor response to fractionated irradiation, the tumor damage produced by single doses of X-rays or neutrons must be studied. Endpoints used include clonogenic cell killing, local tumor control ("cure"), and tumor growth delay. The 50\% tumor control dose (TCD50) with $95 \%$ confidence limits is $2360(1710,3100)$ rads of $250 \mathrm{kVp} X$-rays and $1350(1230,1475)$ rads of neutrons, yielding a neutron RBE of 1.8 for local control. For tumor growth delay in reaching $5 x$ starting volume, the neutron $R B E$ for single fractions is 2.3 to 2.4 over an $\mathrm{X}$-ray dosage range of 750-1700 rads. Since some tumors regress permanently at doses above 1700 rads, growth delay cannot be meaningfully measured at higher doses. The disagreement of RBE's for local control and for growth delay has been reported in other tumor systems, pointing out that delay is often not a relevant endpoint. Experiments are still in progress to determine clonogenic cell survival for EMT-6 cells irradiated in vivo with X-rays and assayed in vitro for colony forming ability.

The survival curve generated by cells excised from tumors treated in vivo with single doses of neutrons has a Do of approximately 150 rads. A dose of 1350 rads (the neutron $T C D_{50}$ ) only reduces cell survival $2 \times 10^{-4}$. This tumor is cured at doses far too low to kill all of the clonogenic cells (estimated to be $2 \times 10^{7}$ in $100 \mathrm{~mm}^{3}$ tumors like the ones used in these experiments). These results suggest that the host's immune response to the tumor is an important factor in cure. Studies by others show that the X-ray TCD50 on $\perp y$ reduces fractiona $\perp$ ce $\perp$ survival to $1 \times 10^{-3}$.

3. Iocal control and growth delay in EMT- 6 solid tumors produced by fractionated $X$-rays and neutrons and by mixed neutron-photon fractionation schemes is currently being evaluated.

Cure and growth delay studies have begun using fractionated X-rays, neutrons and mixed neutron-photon fractionated irradiation. For 5 fractions given in 5 days, the neutron RBE for growth delay ranges from 3.1 to 3.3 . For 2 fractions of neutrons plus 3 fractions of $x$-rays in 5 days, in the sequence $n-n-x-x-x$, the test radiation RBE is 1.4 for growth delay. The TCD 50 ( 958 confidence limits) for 5 fractions of $X$-rays in 5 days is $3450(2820,4219)$ rads and for 5 fractions 
of neutrons is $1350(1080,1688)$ rads. This gives a neutron RBE of 2.6 for local tumor control. As with single doses, the RBE for local control is less than that for growth delay. The neutron $T C D_{50}$ is the same for 1 and 5 fractions. While this is not inconsistent with the virtual absence of a shoulder on the in vivo cell survival curve, it is inconsistent with the repopulation which must occur between fractions. It is possible that in the 5-fraction experiment, some spontaneous regressions occurred in this moderately antigenic tumor. schemes.

Curative dose experiments are in progress; using mixed fractionation

4. The effect of neutron or $X$-irradiation of mouse lungs on the seeding of intravenously injected EMT-6 tumor cells is currently being investigated. The role of the host's immune system in preventing this tumor growth as well as the animal's response to immunosuppression following the whole body irradiation is also being assessed. Initial experiments have employed mice $X$-irradiated to the lungs. These studies have shown that the number of lung tumors 18 days after intravenous injection of 4000 EMT- 6 tumor cells increases with increasing dose to the lungs up to $2000 \mathrm{R}$, the highest dose used. X-irradiation was given 24 hours earlier. The results indicate that with EMT-6 tumor, as with other experimental mouse tumors, localized irradiation of a suspectible organ increases the "takes" of injected tumor cells. Studies utilizing whole body irradiation as well as lung exposure show that immunosuppression further enhances tumor cell seeding. Studies are now in progress to investigate the duration of the local lung effect after irradiation as well as the dose at which the effect reaches saturation, if this occurs at all. The studies will then be repeated with neutron irradiation to determine a neutron $\mathrm{KBE}$ for enhancement of tumor takes in irradiated lung. These studies are of interest if one plans to prophylactically treat a portion of the patient's body suspected of containing tumor. If small doses can enhance seeding of circulating tumor cells in some tumor-bearing organs, this must be weighed against the potential benefits. (These studies are being conducted by an M.S. candidate in Radiological Sciences, Michael Brown.)
* Supported by the National Cancer Institute, Grant No. CA-12441 and Food $\varepsilon$ Drug Administration, Grant No. RL-00071-13.
+ Division of Radiation Oncology, Department of Radiology, University of Washington.

\subsection{Fast Neutron Beam Radiotherapy -- Radiobiology*}

G.M. Christensen ${ }^{\dagger}$, J.P. Geraci ${ }^{\dagger}$, and K.L. Jackson ${ }^{\dagger}$

The major objective of this program is to obtain data which will permit mure effective application of fast neutrons in radiotherapy using the University of Washington cyclotron. For this purpose the relative biological effectiveness, $\mathrm{RBE}$, for oral death from head irradiation was measured. Mice develop recognizable mucosal ulceration in the oral cavity within one week after neutron exposure. Death of the animal, apparently from starvation, occurred 7 to 20 days postexposure. The $\mathrm{LD}_{50}$ for neutron and $\mathrm{X}$-rays and the $\mathrm{RBE}$ are as follows: 
1

2

3
$\mathrm{X}$-ray

Neutron

X-ray

Neutron

X-ray

Neutron
1835

865

2.1

$2400^{\circ}$

1100

2.2 .

2550

1125

2.3

A study was carried out to determine if there is a difference in RBE for reproductive death for cells in log phase of growth and for cells in plateau phase of growth. The KBE's for different levels of survival for plateau- and log-phase cells were determined to be as follows:

\begin{tabular}{lcc} 
Growth Phase & Survival (8) & RBE \\
\cline { 2 - 3 } $\log$ & 50 & 2.87 \\
& 10 & 2.09 \\
Plateau & 1 & 1.98 \\
& 50 & 1.80 \\
& 10 & 1.65 \\
& 1 & 1.58
\end{tabular}

The data clearly reveal that the relative potency of neutrons for reproductive death is much less for plateau cells than log cells.

Decrease in mouse testes weight and DNA content 28 days post-exposure were used as indices for intercomparison of the biological effectiveness of the University of Washington neutron beam with that of other fast neutron teletherapy facilities in the United States and England. The results show that the University of Washington $21 \mathrm{MeV}$ neutron beam is 118 less effective in producing testicular damage than is the Hammersmith Hospital $16-\mathrm{MeV}$ beam in England, and is $6 \%$ and $17 \%$ more effective in producing testicular damage than are the $35-\mathrm{MeV}$ beam at Naval Research Laboratory in Washington; D.C. and the Texas AEM 50-MeV beam, respectively.

In the past year, Dr. Paul Todd of Penn State University and Dr. Eric Hall of Columbia University have visited the University of Washington cyclotron to do similar studies with other tissue culture systems. The results of these studies are not yet available.

* Supported by National Cancer Institute Grant No. CA-12441

+ Division of. Radiation Biology, Department of Radiology, University of Washington. 
17.4 Fast Neutron Beam Radiotherapy -- Clinical Program*

H.C. Berry ${ }^{\dagger}$, A.J. Gerdes ${ }^{\dagger}$, D.W. Hebard ${ }^{\dagger}$, and R:G. Parker ${ }^{\dagger}$

Between September 10, 1973 and January 1, 1976, 134 patients entered the study. During this time, our interest was limited to cancers arising in the head and neck.

The objectives of our programs were: (a) to determine short-term and longterm normal tissue tolerances; (b) to quantitate tumor responses; (c) to develop treatment methods; (d) to determine optimum pattems of treatment application; (e) to develop and initiate national cooperative protocol studies.

Three Phase I studies were reported:

1. Fast neutron beam radiation therapy of glioblastoma multiforme.-- The first 21 patients who received supratentorial fast neutron beam imadiation did not appear to have longer or better survival than that expected following conventional photon radiation therapy. However, in 7 patients studied postmortem, tumor destruction was more complete than expected following conventional photon radiation therapy. A Phase II study using a mixed beam, 2 increments of $60 \mathrm{rad}_{\mathrm{n} \gamma}$ of fast neutrons plus 3 increments of 180 rad of $60^{\circ}$ o photons, followed by a fast neutron "boost" to the tumor is in progress. The Phase I study was reported to the American Radium Society, San Juan, Puerto Rico, December 1974, and published in the American Journal of Roentgenology (in press).

2. Fast neutron beam radiation therapy of metastatic cervical adenopathy. -- 36 patients were irradiated for metastatic cervical adenopathy from cancers arising in the oral cavity or pharynx. In approximately $50 \%$ of the patients, the tumor was controlled even when extensive at the time of treatment. These results are comparable to the best results reported in the literature. This Phase I study was reported at the 1975 meeting of the American Society of Therapeutic Radiologists, San Francisco, California, and published in Cancer (in press).

3. Assessment of normal tissue tolerance. -- Treatment-produced shortterm sequellae were evaluated in all patients. Skin and mucosal reactions were evaluated in all patients. Skin and mucosal reactions were moderately severe but all healed and did not permanently interrupt treatment. No serious reactions were noted in the brain, spinal cord, eye, teeth, bone or cartilage. Long-term tolerance is being assessed in survivors.

- Various patterns of treatment and doses continue to be studied. Currently patients are treated either with 4 fractions of fast neutrons weekly or with 2 fractions of fast neutrons plus 3 fractions of ${ }^{60} \mathrm{Co}$ photons weekly.

A versatile chair-couch for patients was constructed and made operational.

Protocols for several different cancers are being developed and should become operational in 1976. All NCI-supported programs are expected to participate. This may appreciably increase the number of patients entering various studies. 
Through the cooperation of the faculty of the Department of Physics and the staff of the cyclotron, access to the cyclotron was increased to permit treatment with 4 increments weekly. This allowed our participation in studies already operational in the M.D. Anderson-TAMVEC and MANTA programs .

+ Division of Radiation Oncology, Department of Radiology, University of Washington.

* Supported by NCI Grant No. CA-12441.

17.5 Total Body Calcium by Neutron Activation Analysis Techniques: Applicability to Bone Wasting Disease, and Space Flight Related Bone Loss C.H. Chesnut $^{\dagger}$, G. Hinn ${ }^{\dagger}$, T.K. Lewellen ${ }^{\dagger}$, R. Murano ${ }^{\dagger}$, and W.B. Nelp ${ }^{\dagger}$

The cyclotron-based determination of total body calcium (TBC) by neutron activation $\left[48 \mathrm{Ca}(\mathrm{n}, \mathrm{Y})^{49 \mathrm{Ca}]}\right.$ is currently utilized in three large studies assessing the effects of various therapies in postmenopausal osteoporosis, the most common bone wasting disease. Two controlled double blind studies involving 50 postmenopausal osteoporotic females each assess response to treatment with either stanozolol (an anabolic steroid) or calcitonin; a third study (in collaboration with the University of Minnesota) will examine the effects of estrogens in $50-60$ osteoporotic females. The primary parameter for assessing response to therapy is serial (every 6 months) quantitation of TBC. These studies should provide definitive information regarding the etiology and therapy of osteoporosis.

In addition to the above studies, the TBC-neutron activation analysis technique continues to be applied to the study of normal bone mass: Five males and females at each decade age 20-90 are having TBC quantitated to provide comparative data for osteoporotic individuals, and to further investigate agerelated bone loss.

Neutron activation analysis techniques are also being applied to the problem of bone loss related to long term space flight, in collaboration with NASA. A new technique, that of TBC determination by quantitation of the radioactive gas Argon-37 in the subject's expired air $\left[40 \mathrm{Ca}(n, \alpha){ }^{37} \mathrm{Ar}\right]$, provides definitive information. Calibration of the technique is by the original TBCneutron activation analysis.

$+\quad$ Division of Nuclear Medicine, University of Washington.

17.6 Nuclear Pharmacy

D.R. Allen ${ }^{\dagger}$

The University of Washington Nuclear Pharmacy was able to utilize the cyclotron for the production of fluorine-18. Specifically, gas targetry $\left[{ }^{20} \mathrm{Ne}(d, \alpha)^{18} \mathrm{~F}\right]$ was developed to produce radiolabeled $\mathrm{NO}^{18} \mathrm{~F}$ which was subsequently utilized to synthesize $18 \mathrm{~F}-5 \alpha$-fluoro-3 $\beta$-hydroxycholestane 6-nitramine acetate which was the subject of Mr. David Hartnett's master thesis within the Radiological Sciences Department.

$\mp$ Division of Nuclear Medicine, University of Washington. 
17.7 Alpha Particle Injection into Reactor Materials

K.R. Garr $^{\dagger}$

Alpha-particle irradiation of fast-reactor cladding and structural material candidates were performed under a program at Atomics Intemational sponsored by ERDA-RDD titled "Advanced Alloy Development Program", Task 17, Contract AT(04-3)824, using the University of Washington's cyclotron. The cyclotron provides a fast and convenient method of introducing large concentrations of helium uniformly into specimens.

This program investigated the effect of helium on the high temperature $\left(700^{\circ} \mathrm{C}\right)$ stress-rupture behavior of advanced alloys, i.e., alloys other than Type 316 stainless steel, that have potential use in liquid-metal-cooled-fast reactors. The test specimens, in the form of tubes, were rotated and water cooled while being irradiated with alpha particles from the cyclotron.

After the cyclotron irradiation was completed, the test specimens had end caps welded onto them in regions that were shielded from the cyclotron beam. Twelve specimens were then placed in a retort, heated to the test temperature, $700^{\circ} \mathrm{C}$, and pressurized, internally stressed, with helium gas. Two specimens were pressurized to the same stress giving six different stress levels for each retort. A second retort containing specimens that had not been alpha-particle irradiated were used as a control for comparison.

The different stress levels produce different failure times in the specimens. A helium effect is obtained by plotting $10 \mathrm{~g}$ stress vs log-time-to-failure for both controls and irradiated specimens and observing if there are changes in the time-to-failure for the irradiated specimens.

A second program on the Advanced Alloy Development Program consisted of injecting helium into a special $\mathrm{Fe}-\mathrm{Cr}-\mathrm{Ni}$ alloy and then irradiating it with protons to study the void formation. This experiment was done in conjunction with several other laboratories to compare swelling in an alloy due to irradiation with different ions.

$\uparrow \quad$ Atomics International, Canoga Park, California.

17.8 Delayed Neutron Spectra Measurements*

G. W. Eccleston ${ }^{\dagger}$ and G.L. Woodruff ${ }^{\dagger}$

The near-equilibrium energy spectra of the delayed neutrons associated with the fast-neutron-induced fission of ${ }^{232} \mathrm{Th},{ }^{233} \mathrm{U},{ }^{235} \mathrm{U},{ }^{238} \mathrm{U}$, and ${ }^{239} \mathrm{Pu}$ have been measured at the University of Washington Nuclear Physics Laboratory.1 The fast source neutrons were provided by focusing a $10 \mathrm{MeV}$ proton beam, generated with the University of Washington Tandem Van de Graaff, onto a thick ( $80 \mathrm{mil}$ ) beryllium target. 2

Measurements were collected with a two-parameter proton-recoil spectrometry system. 3 Two identical detectors, $3.01 \mathrm{~cm}$ diameter and $15.24 \mathrm{~cm}$ active length, 
one filled with $4 \mathrm{~atm}$ hydrogen (resolution $=5 \%$ ) and the other with 5 atm methane (resolution $=8 \%$ ) were used. The hydrogen filled detector measured protons from 20 to $250 \mathrm{keV}$ while the high energy band, from 200 to $1500 \mathrm{keV}$, was collected with the methane filling. Two parameter data were unfolded to neutron spectra by first collapsing to one parameter proton recoil ionization spectra followed by frequency filtering, 4 to improve the statistical properties, and then differentiating. The complete spectrum is a composite of four overlapping energy intervals.

The detector and sample were encased in lead to decrease the measured gamma component produced by the beryllium target and the sample. The detector shield was $20.32 \mathrm{~cm}$ long by $10.16 \mathrm{~cm}^{2}$ with a $4 \mathrm{~cm}$ diameter hole centered in the squared end to accomodate the detector. A total of $7.8 \mathrm{~cm}$ of lead separated the detector and isotope. Recent data 5 indicate that no appreciable bias is introduced by lead shields of comparable thicknesses.

Each of the five isotopes was measured during a 48 hour continuous run. Data was collected using a repetitive sequence consisting of a 1.0-sec 1riadiatiun, $0.1-\mathrm{sec}$ delay and a 10.-sec counting period. Electrostatic deflection of the proton beam (on/off ratio > 105) provided rapid shutoff of the fast suluce neu* trons, permitting a short delay time prior to counting. The $235 \mathrm{U}$ delayed neutron group yields for this measurement, computed using data from Keepin 6 and compared to his equilibrium yields, are as follows:

\begin{tabular}{cccc}
\hline GROUP & MEASURED & KEEPIN & * DIFFERE'NCE \\
\hline 1 & 0.040 & 0.038 & +5.26 \\
2 & 0.223 & 0.213 & +4.69 \\
3 & 0.196 & 0.188 & +4.25 \\
4 & 0.422 & 0.407 & +3.68 \\
5 & 0.109 & 0.128 & -14.8 \\
.6 & 0.010 & 0.026 & -61.5 \\
\hline
\end{tabular}

The delayed neutron spectrum for the ${ }^{235} \mathrm{U}$ isotope is shown in Fig. 17.8-1. This spectrum, in addition to our other measurements, exhibits fine structure in agreement with previous comparisons between the observed peaks from other experiments. ${ }^{3}$ Our data compare closely with the thermal delayed neutron spectrum measurements of Sloan and Woodruff.' 3 A recent comparison belweeil measured delayed neutron spectra illustrating spectral differences was published. 7 These results indicate that unresolved differences still remain in the low energy portion of delayed neutron spertira.

* Work supported by AEC Contract \#A.T. (45-1)-2225.

+ Department of Nuclear Engineering, University of Washington.

1. Nuclear Physics Laboratory Annual Report, University of Washington (1975), p. 218.

2. E. Tuchilin and G.D. Kohler, Health Phyeics, Vol. 1 (1958).

3. W. Robert Sloan and Gene L. Woodruff, Nucl. Sci. Eng. 55, 28 (1974).

4. W. Robert Sloan and Gene L. Woodruff, Frequency Filtering of Proton-Recoil Data, to be published in Nucl. Instrum. Methods. 


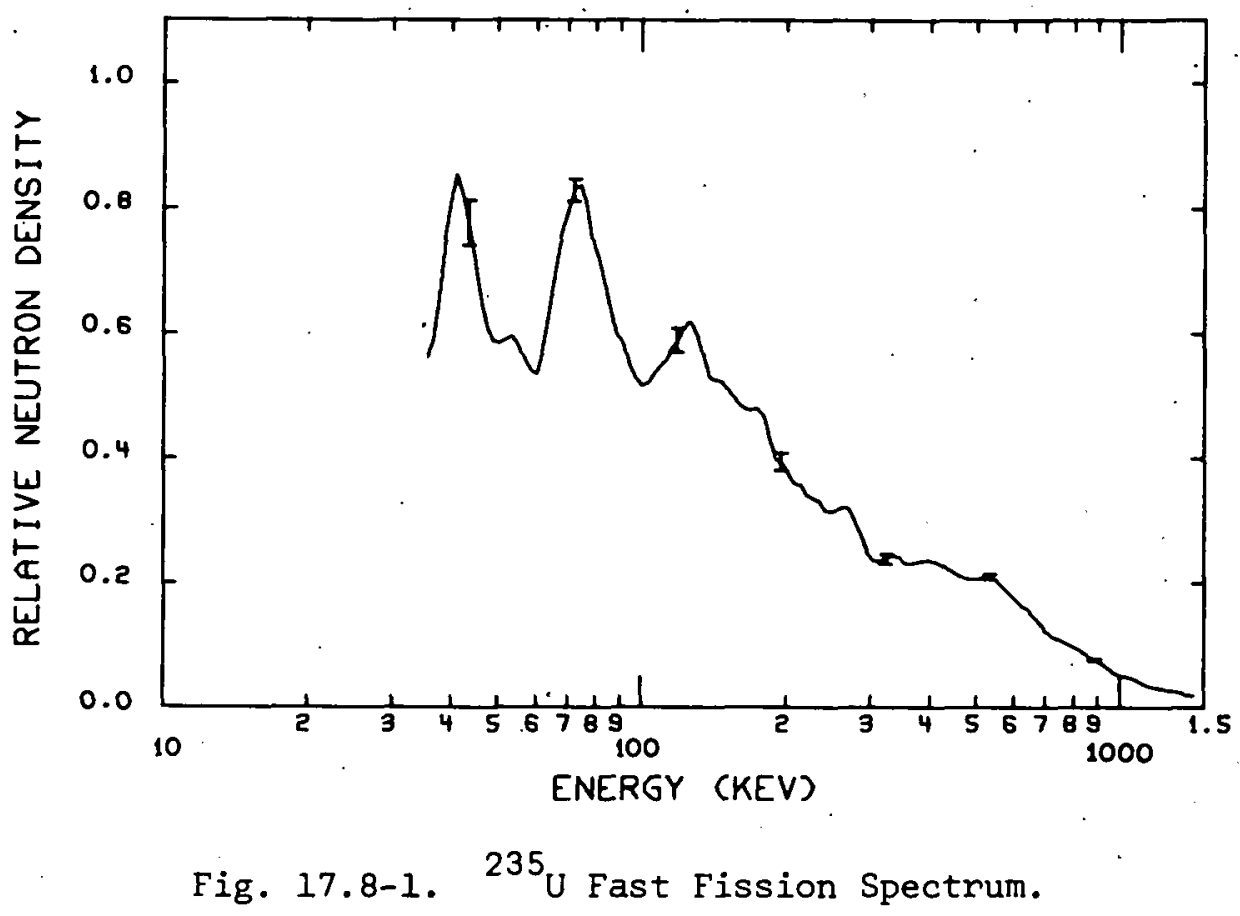

5. G. Fieg, M. Lalovic and G. Woodruff, Nucl. Sci. Eng. 58, 260 (1975).

6. G.R. Keepin, Physics of Nuclear Kinetics (Addison-Wesley 1965), Chapter 4.

7. G.W. Eccleston and G.L. Woodruff, Delayed Neutron Spectra from 232 Th, ${ }^{233} \mathrm{U}, 235 \mathrm{U}, 238 \mathrm{U}$ and $239 \mathrm{Pu}$, to be published in Transactions of the American Nuclear Society. -

17.9 Optical Properties of the Alkali Halides*

F.P. Carlson ${ }^{\dagger}$ and M.J. Intlekofer ${ }^{\dagger}$

We have been continuing to use the University of Washington cyclotron in our investigation of some of the optical properties of the alkali halides. When alkali halide crystals are subjected to crystal lattice damage, color centers are formed which exhibit polarization sensitive absorption characteristics. In addition, the color centers are re-orientable, which provides a means for the writing and non-destructive reading of stored information. This makes them useful as input masks in coherent optical processing systems, as the sensitive medium in holography, and as the propagating medium in thin film optics.

We have found that alpha particle bombardment in the cyclotron provides the optical density and penetration necessary for our work, and in addition, the uniformity and persistence of the coloration is excellent. In a typical experimental session, a single crystal or an evaporated thin film of $\mathrm{KCl}$ is exposed to a diffuse beam of high energy alpha particles. The sample is then scanned with a spectrophotometer and reorientation and propagation studies are performed.

* Work supported by U.S. Navy, Contract No. 0014-67-A-0103-0024, Task Order NR 350-005.

$\pm \quad$ Department of Electrical Engineering, University of Washington. 
17.10 Hyperfine Strưcture Anomalies in the Cadmium Isotopes ${ }^{1}$

B. Geelhood ${ }^{\dagger}$ and M.N. McDermott ${ }^{\dagger}$

Attempts to observe nmr signals from optically oriented samples of ${ }^{105} \mathrm{Cd}$ and $111 \mathrm{~cd}$ produced at the cyclotron were unsuccessful. An estimate of the signal expected based on the observed activity of the two isotopes and the nmr signals from ${ }^{107} \mathrm{Cd}$ produced in the same target indicated the experiment was marginal at best.

The hyperfine anomalies for all the cadmium isotopes have been re-evaluated. The results are generally in poor agreement with predictions based on a configuration mixing model. The most striking aspect of the analys is is the indication that the magnetization in the $I=11 / 2$ isomers is distributed at smaller radii than that in the $I=1 / 2$ isotopes.

$\dagger$ Department of Physics, University of Washington. $111 m_{C d}$, and $111 m_{C d}$, Ph.D. Thes is, University of Washington (1975), unpublished.

17.11 The Role of Compound Nuclear Angular Momentum in Determining Fission Fragment Angular Momentum

W. Loveland $^{\dagger}$ and A. Schilling ${ }^{\dagger}$

There are generally conceded to be three sources for the relatively high angular momenta of the primary fragments in nuclear fission. They are: (a) an electrostatic "post-scission" torque that each fragment exerts on the other fragment. This torque arises from a non-axial splitting of the neck of the fissioning nucleus and results in the fragments rotating in opposite directions. (b) a quantal "pre-scission" excitation of rotational states in the nascent fragments. (c) a portion of the initial compound nuclear angular momentum.

We have undertaken a series of experiments to explore the role of (c) above in determining the primary fragment angular momentum. We have formed the same compound nucleus, ${ }^{239} \mathrm{Pu}$, at the same excitation energy, $E^{*}=31.1 \mathrm{MeV}$, in two different ways: (a) $235_{\mathrm{U}}+36.7 \mathrm{MeV}$-particles and (b) $237_{\mathrm{Np}}+22.0 \mathrm{MeV}$ deuterons. For these two cases in which the compound nuclear angular momenta are $14 \hbar$ and $8 \hbar$, respectively, we are measuring the $134 \mathrm{Cs}$ isomer ratio. From this $134 \mathrm{Cs}$ isomer ratio,

$$
\frac{\sigma_{131}+m_{C s}(J, \pi=8-)}{\sigma_{134 g_{C s}(J, \pi=4+)}},
$$

we want to calculate, using modern level density prescriptions, the average primary fragment angular momentum for each case. We have performed irradiations of $5 \mathrm{mg} / \mathrm{cm}^{2} 235 \mathrm{U}$ and ${ }^{237} \mathrm{~Np}$ targets at the University of Washington cyclotron. Cs fission product activities were isolated from Al catcher foils surrounding 
the target using the radiochemical separation procedure of Glendenin and Nelson. ${ }^{1}$ The $134 \mathrm{~m}_{\mathrm{Cs}}$ activity was measured immediately following chemical preparations by $\gamma$-ray singles counting with a large volume Ge(Li) detector. We are currently waiting for the large amounts of $136 \mathrm{Cs}$ found in the sample to decay so that we may assay the 2.1 year $134 \mathrm{~g}$ S activity and compute the isomer ratio.

$\dagger$ Department of Chemistry, Oregon State University, Corvaliis, OR. 1. L.E. Glendenin and C.M. Nelson, in Radiochemical Studies: The Fission Products, C.D. Coryell and N. Sugarman, ed., National Nuclear Energy Series, Division IV, Vol. 9, p: 1642 (McGraw-Hill, New York, 1951).

17.12 Gamma-Ray Angular Correlations in Odd-Mass Nuclei

K.S. Krane ${ }^{\dagger}$ and J. Shobaki ${ }^{\dagger}$

The understanding of systemtatic details of nuclear structure requires detailed knowledge of the electromagnetic transition moments between low-lying nuclear states. In order to obtain such information for states in odd-mass nuclei in the mass region of $A=100$, we have begun a series of measurements of gamma-ray angular correlations following the radioactive decays of cyclotronproduced activities. These measurements are expected to yield new insights into some systematic trends of nuclear structure.

+ Department of Physics, Oregon State University, Corvallis, OR.

17.13 The Helium Ion-Induced Fission of Enriched Isotopes of Mercury and

Tungsten at Moderate Bombarding Energies

E.F. Neuzil ${ }^{\dagger}$

This represents the final experimental results of a series of investigations over the past 12 years concerning the charged particle induced fission of isotopes below polonium on the periodic chart. Again, as before, the bombarding particles were 41 and $65 \mathrm{MeV}$ helium and the targets in this case were enriched isotopes of mercury and tungsten. The total fission cross sections ( $\sigma_{f}$ ) for the mercury isotopes 198, 199, 200, 201, 202, and 204 at a bombarding energy of $41 \mathrm{MeV}$ were $265,315,365,164,85.7$ and $73.1 \mu \mathrm{b}$ respectively. A single determination of the fission cross sections at $65 \mathrm{MeV}$ for $198_{\mathrm{Hg}}$ and $199_{\mathrm{Hg}}$ were $37,800 \mu \mathrm{b}$ and $25,200 \mu \mathrm{b}$ respectively. These results as well as the widths at half maximum $\left(W_{1 / 2}\right)$ of the fission fragment mass distribution curve were consistent with previous trends; the widths were approximately 20 amutl amu. The values of the $\sigma_{f}$ of the $41 \mathrm{MeV}$ helium ion-induced fission of isotopes 182, 183, 184 and 186 of tungsten were estimated to be $0.56,0.90,0.47$ and $0.27 \mu \mathrm{b}$ respectively.

+ Department of Chemistry, Western Washington State College, Bellingham, WA. 
17.14. The ${ }^{89} \mathrm{Y}(\mathrm{p}, \mathrm{\gamma})^{90} \mathrm{Zr}$ Reaction below the Giant Dipole Resonance

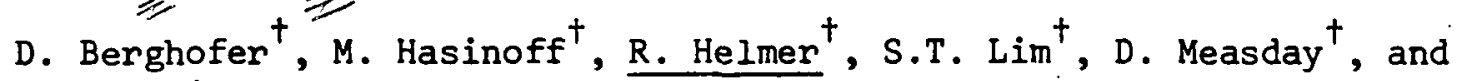

T.A. Trainor Last year in this report we presented a preliminary assessment of our 90
search for isoscalar E2 strength located below the Giant Dipole Resonance of $\mathrm{Zr}$ via the $89 \mathrm{Y}(\mathrm{p}, \mathrm{\gamma})^{90} \mathrm{Zr}$ reaction. This strength, presumably resulting from a Giant Isoscalar Quadrupole Resonance, has been seen in inelastic scattering from $90 \mathrm{Zr}$ of electrons, 1 protons, 2 He, 3 and ${ }^{4} \mathrm{He} .4$ The peak is located at an excitation in $90 \mathrm{Zr}$ of about $14 \mathrm{MeV}$, and has a width of 4 or $5 \mathrm{MeV}$. Here we present the results of measurements of the angular distribution of the gamma rays leading to the ground state at twelve energies from $3 \mathrm{MeV}$ to $7 \mathrm{MeV}\left(\mathrm{E}_{\mathbf{X}}=11.3 \mathrm{MeV}\right.$ to 15.1 $\mathrm{MeV})$, and the results of polarization measurements at four energies in this interval.

The target was a self-supporting foll of ${ }^{89} \mathrm{Y}$ of thickness $4.2 \mathrm{mg} / \mathrm{cm}^{2}$ and the gamma rays were detected in a $10^{\prime \prime} \times 10^{\prime \prime} \mathrm{NaI}$ detector with a plastic anti-

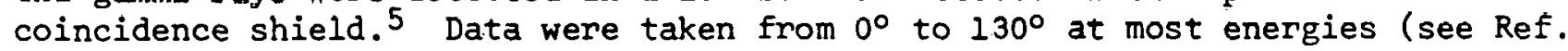
6 for an example of an angular distribution). The distributions were fitted by a Legendre polynomial expansion

$$
W(\theta) \sim A_{0}\left[1+\sum_{i=1}^{L} a_{i} P_{i}(\cos \theta)\right] \text {. }
$$

The $a_{i}$ can be related to the amplitudes and phases of the partial waves contributing to the reaction $\left(\ell_{j}=s_{1 / 2}\right.$ and $d_{3 / 2}$ for $E l$ capture, $\ell_{j}=p_{3 / 2}$ and $f_{5 / 2}$ for $E 2$ capture).

Figure 17.14-1 shows the results of fitting the angular distributions lo $\mathrm{L}_{\max }=3$. It is seen that the $\mathrm{a}_{2}$ term dominates. This term depends mainly on dipole radiation, although it also contains small incoherent contributions from quadrupole radiation. The presence of quadrupole radiation is expected to show up most strongly in the $a_{1}$ and $a_{3}$ coefficients which arise from dipole-quadrupole interference. There is a hint of a positive al coefficient and a negative $a_{3}$ coefficient, in accordance with other radiative capture experiments. ${ }^{2}$ The pure quadruple term, $a_{4}$, was not included in this analysis because in general, its inclusion only served to increase the errors on the lower order coefficients with no compensating improvement to the fits. Hence the overall picture was obscured.

In order to extract unambiguously the partial wave amplitudes, it is necessary to measure the asymmetry produced in the gamma radiation when the reaction is initiated by polarized protons. 8 The asymmetry can be fitted by a function of the form 9

$$
\left(\sigma_{\uparrow}(\theta)-\sigma_{t}(\theta)\right) / 2 \mathrm{p}=A_{0} \sum_{i=1}^{\max _{i}} \mathrm{~b}_{i} \mathrm{P}_{i}^{1}(\cos \theta)
$$

where the arrows represent the proton spin direction (up or down), $p$ is the proton beam polarization (normal to the reaction plane), and the $P P_{i}(\cos \theta)$ are the associated Legendre polynomials. The $b_{i}$ depend on the partial wave amplitudes in the same way that the $a_{i}$ do, except that only interfering waves contribute. 
The asymmetry was measured at four energies and fitted to the expression. above. The results are summarized in. Table 17.14-1. The b4 coefficient was not

Table 17.14-1. $\sigma\left(\gamma_{E 2}, p\right)$ obtained by detailed balance, and b coefficients from ${ }^{89} \mathrm{Y}(\overrightarrow{\mathrm{p}}, \gamma){ }^{90} \mathrm{Zr}$.

$\begin{array}{lccccc}\mathrm{E} \rightarrow \mathrm{p} & \mathrm{b}_{1} & \mathrm{~b}_{2} & \mathrm{~b}_{3} & \mathrm{~b}_{4} & \sigma\left(\gamma_{E 2}, \mathrm{p}\right) \\ 4.2 & -.01 \pm .01 & -.03 \pm .01 & -.01 \pm .01 & .01 \pm .01 & 30 \pm 23 \mu \mathrm{b} \\ 4.8 & .04 \pm .02 & -.04 \pm .02 & .01 \pm .02 & .00 \pm .02 & 46 \pm 39 \mu \mathrm{b} \\ 6.15 & .00 \pm .02 & .05 \pm .02 & -.01 \pm .01 & .01 \pm .01 & 9 \pm 16 \mu \mathrm{b} \\ 6.8 & .03 \pm .02 & .11 \pm .02 & .01 \pm .02 & .02 \pm .02 & 15 \pm 15 \mu \mathrm{b}\end{array}$

included in the analysis because the errors on the data points were large enough (the results of subtracting two nearly equal large numbers) that its inclusion did not significantly alter the lower order coefficients.

We have made a preliminary extraction of the partial wave amplitudes and phases, and hence the relative El and E2 cross sections, from the $a$ and $b$ coefficients using a computer program written for this purpose by $\mathrm{J}$. Bussoletti of this Laboratory. By making use of the differential cross section measurements of Hasinoff et al. 10 above $6 \mathrm{MeV}$ and the $90^{\circ}$ yield curve of Mason et al. 11 below $6 \mathrm{MeV}$, we have obtained the total E2 cross section as a function of energy. The last column of Table 17.14-1 lists the total $\bar{E} 2$ photodisintegration cross section obtained by applying detailed balance to the capture cross sections.

Because the errors are so large it is difficult to compare these results to what would be expected on the basis of various sum rules, or to say whether there is any indication of concentrated E2 strength in this region. However, we find approximately the upperilimit of the energy-weighted sum $\int \sigma(E 2) d E / E^{2}$ to be $0.9 \mu \mathrm{b} / \mathrm{MeV}$ while the sum rule limit is $2140 \mu \mathrm{b} / \mathrm{MeV}$. Thus about $0.6 \%$ of the sum rule is exhausted, which is of the
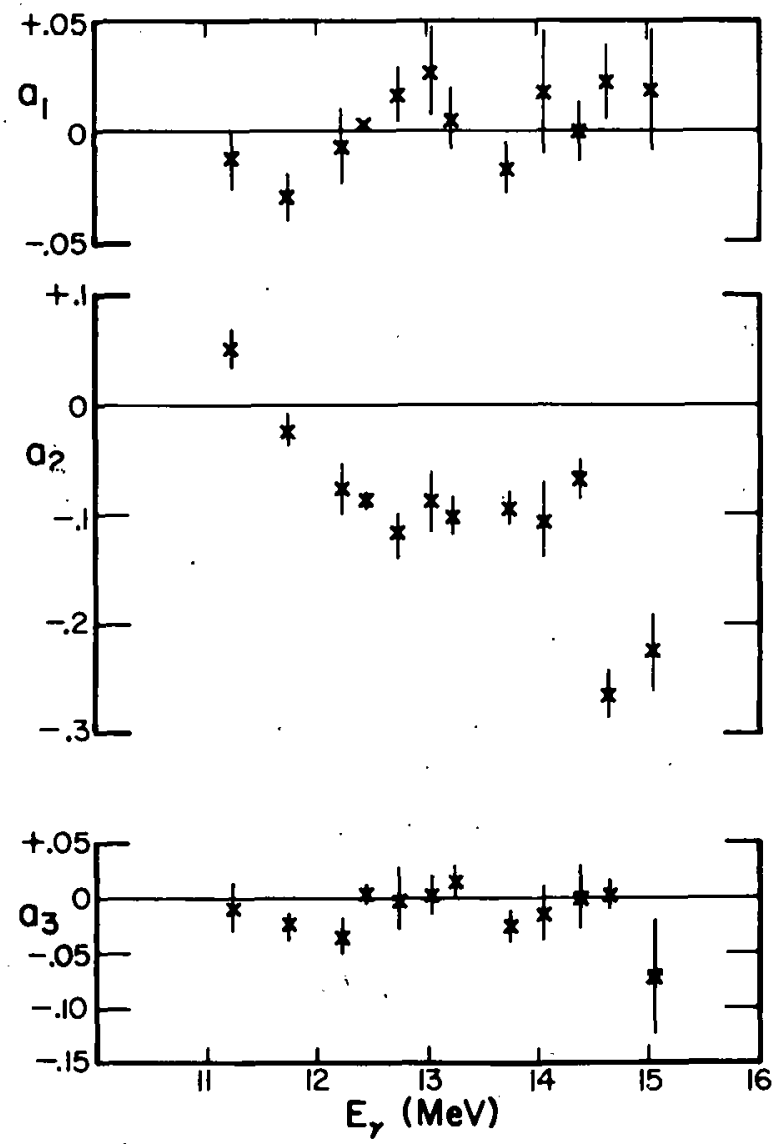

Fig. 17.14-1. Angular distribution coefficients $a_{1} \rightarrow a_{3}$ from unpolarized proton capture. 
same order as has been found for other nuclei: 7

Further analysis is in progress.

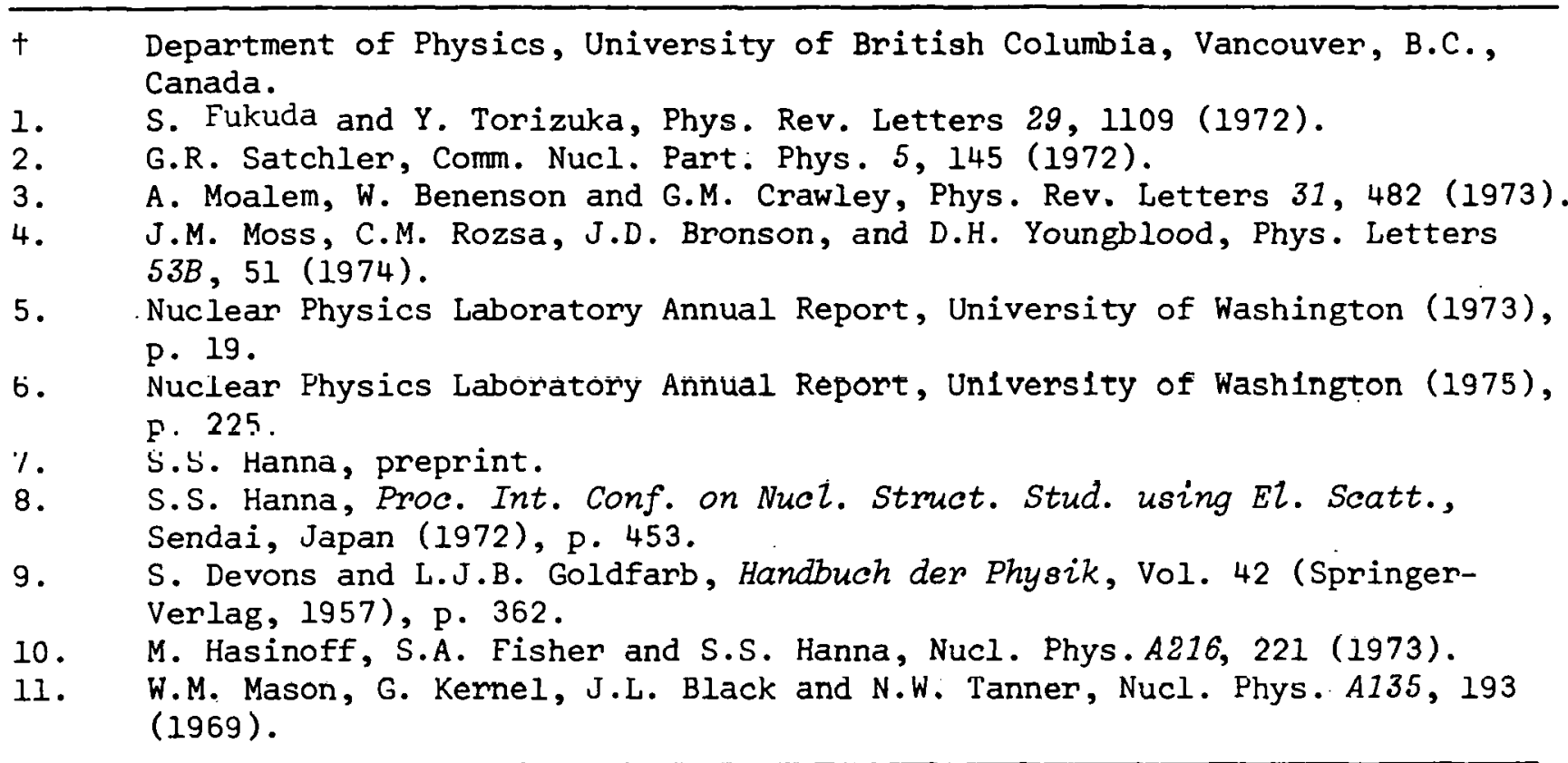

17.15 Radiative Proton Capture by Carbon-12

D. Berghofer ${ }^{\dagger}$, K. Ebisawa, M.D. Hasinoff ${ }^{\dagger}, \underline{\text { R. Helmer }}^{\dagger}$, S.T. $\operatorname{Lim}^{\dagger}$,

D.F. Measdayt, and T.A. Trainor

Our survey of the ${ }^{12} \mathrm{C}(\mathrm{p}, \gamma)^{13} \mathrm{~N}$ reaction using unpolarized protons is now completed and the results have been accepted for publication. 1 The energy regions covered were approximately $8.5 \mathrm{MeV}$ to $24.4 \mathrm{MeV}$ for the $\left(p, \gamma_{0}\right)$ reaction, $19.8 \mathrm{MeV}$ to $24.4 \mathrm{MeV}$ for the $\left(p, \gamma_{1}\right)$ and $\left(p, \gamma_{2}+3\right)$ reactions, and from threshold to $24.4 \mathrm{MeV}$ for the $\left(p_{,} p^{\prime} \gamma_{12.7}\right)$ and $\left(p_{,} p^{\prime} \gamma_{15.1}\right)$ reactions. The data consisted of $90^{\circ}$ yield curves for all these reactions and several angular distributions for the studies of the ground state and inelastic gamma transitions.

Most of this work was reported in last year's Annual Report, ${ }^{2}$ but here we show the yield curves for the transitions to the first excited state (Fig. 17.15-1) and to the sum of the second and third excited states (Fig. 17.15-2). These two states lie only $40 \mathrm{keV}$ apart and cannot be resolved by our apparatus. The results for $\gamma_{2+3}$ have been combined with the results of f'isher et al. 3 so that the region of investigation for. this transition now extends from $19.8 \mathrm{MeV}$ to $40 \mathrm{MeV}$. For energies less than $19.8 \mathrm{MeV}$, the gammas to the first three excited states are lost in a sea of piled-up pulses and inelastic gamma rays.

The giant resonance for $\gamma_{1}$ is centered near an excitation energy of $22 \mathrm{MeV}$ $\left(E_{\gamma}=19.6 \mathrm{MeV}\right)$, and for $\gamma_{2+3}$ near an excitation energy of $26 \mathrm{MeV}\left(E_{\gamma}=22.5 \mathrm{MeV}\right)$. Neither of these peaks seem to correspond in a simple way to the peak in the $Y_{0}$ cross-section at $20.8 \mathrm{MeV}$. 


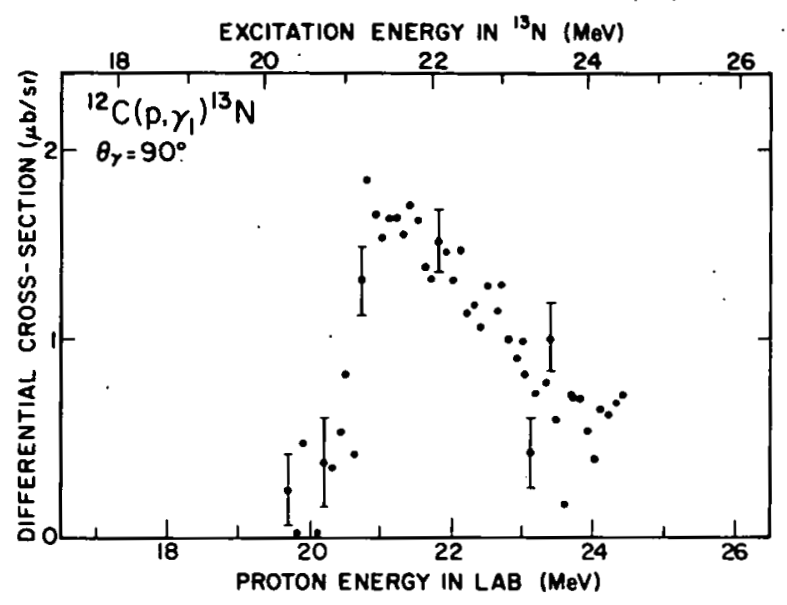

We are currently extending our survey of the $\left(p, Y_{0}\right)$ reaction using polarized protons. The purpose here is two-fold; first to investigate (in conjunction with a program being carried out at this Laboratory for other nuclei ${ }^{4}$ ) the behavior of the radiation (presumed to be E2) which gives rise to the non-vanishing al coefficient in the angular distribution, 2 and second to explore further the nature of the dips seen in the yield curve in the region of the pygmy resonance. 5 We have completed measurements at $11.2 \mathrm{MeV}$ and $13.5 \mathrm{MeV}$, which are located on the high energy sides of these dips. The yield and asymmetry curves together with the extracted Legendre and associated

Fig. 17.15-1. Differential cross-section for the $90^{\circ}$ yield of capture gamma rays leading to the first excited state of $13 \mathrm{~N}$ at $2.37 \mathrm{MeV}$. In addition to the relative errors shown, there is an uncertainty in the overall normalization of $\pm 20 \%$.

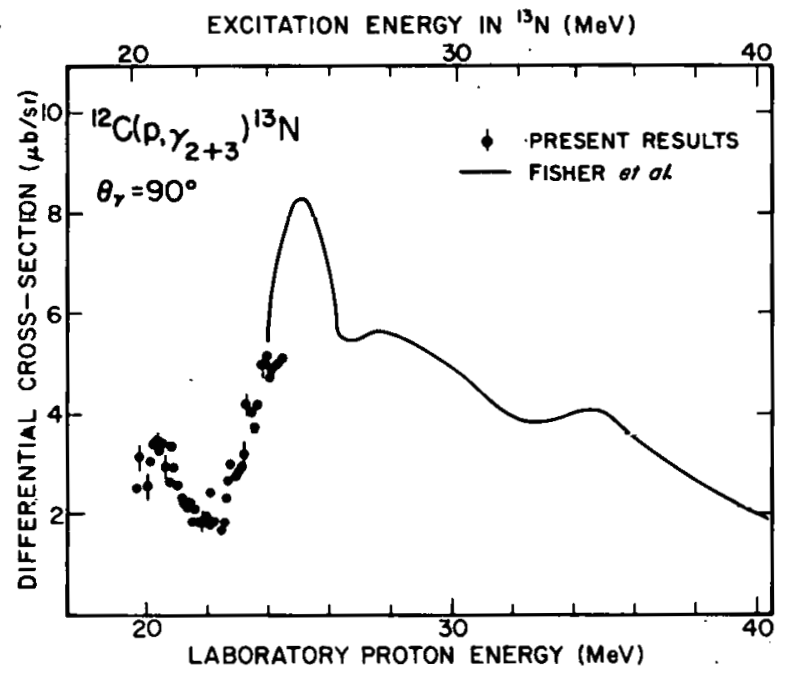

Fig. 17.15-2. Differential cross-section for the $90^{\circ}$ yield of capture gamma rays leading to the unresolved second and thind excited states of $13_{\mathrm{N}}$ at 3.51 $\mathrm{MeV}$ and $3.56 \mathrm{MeV}$. In addition to the relative errors shown, there is an uncertainty in the overall normalization of $\pm 20 \%$.

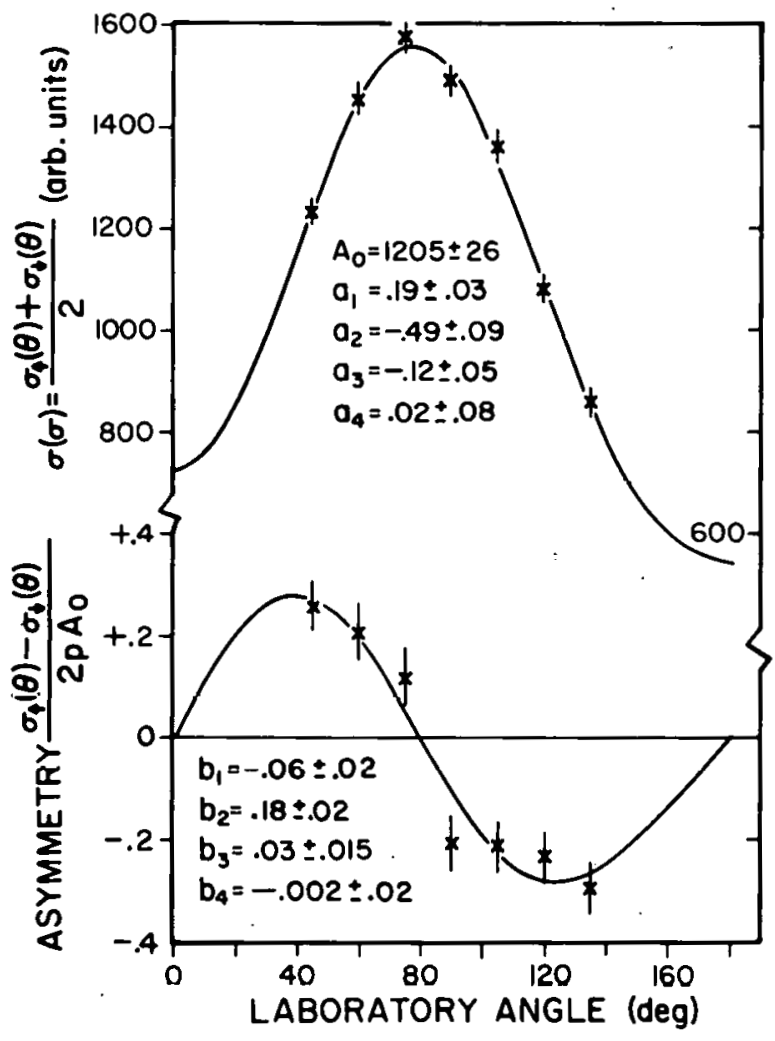

Fig. 17.15-3. Yield and asymmetry for the capture gamma ray leading to the ground state of $13_{\mathrm{N}}$. The errors are statistical and include a small contribution from background subtraction. 
Legendre polynomial coefficients are shown in Fig. 17.15-3 for $E_{p}=13.5 \mathrm{MeV}$. The coefficients can be related to the amplitudes and phases of the partial waves $\left(l_{j}=s_{1 / 2}, P_{3} / 2, d_{3 / 2}, f_{5 / 2}\right.$ for El and E2 capture) taking part in the reaction, 6 and this analysis is currently being carried out.

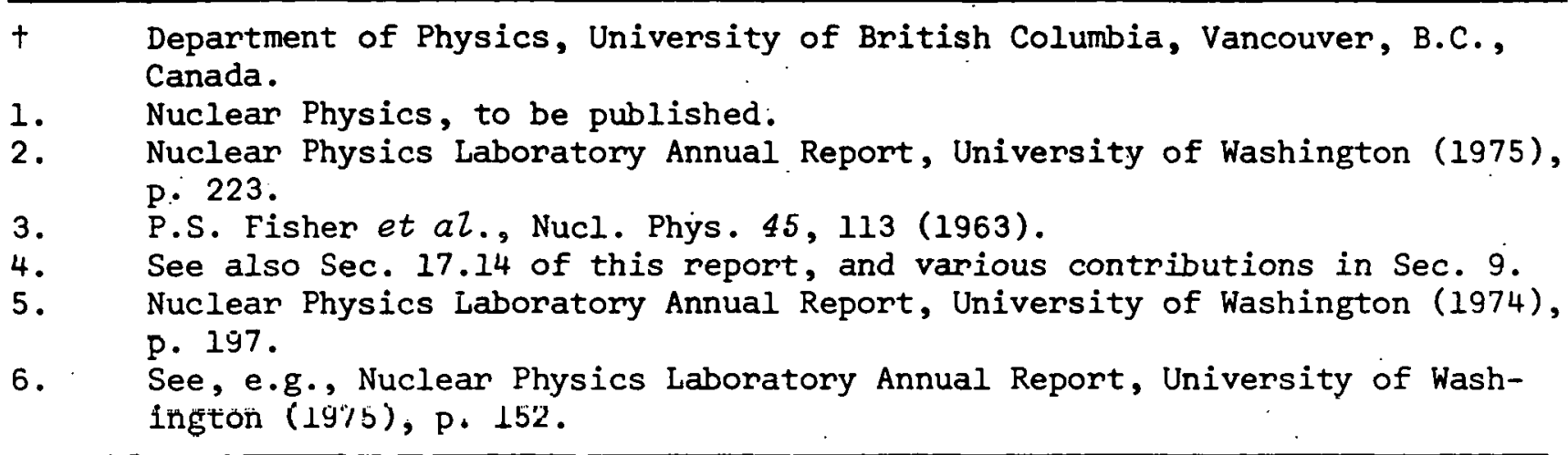

17.16 Plastic Track Detector Calibration

H.B. Knowles $^{\dagger}$, F.H. Ruddy ${ }^{\dagger}$, and G.E. Tripard ${ }^{\dagger}$

Since 1974 a research group at Washington State University has been employing plastic track detectors for the measurement of heavy ions emitted by high-energy negative pions and captured negative pions when either interacts with tissue-resident nuclei. Starting in 1975, track detector measurements of heavy ions produced by therapeutic fast neutron beams has been initiated.

The most sensitive of the plastics employed (Kodak Pathe CA 80.15 cellulnse nitrate) had never been completely calibrated for range and etch rate against beams of heavy ions of known energy. This is especially significant because the WSU group employs a much lower etch temperature than do other groups using track detectors to measure heavy ions of very low energy. During the etch of several cellulose nitrate films exposed to low-energy 160 ions at Los Alamos, a previously unremarkable etch parameter, etch induction time, was found to show a quasilinear dependence on incident particle energy. The existence of a third etch parameter, other than range and etch rate, would provide a simple method for measuring energy, change, and mass (isotope) of an incident heavy ion, thus extending useful domain of heavy ion spectroscopy down to a region of $\sim_{0.1-0.2}$ $\mathrm{MeV} / \mathrm{amu}$ specific kinetic energy. This would extend the domain of heavy ion measurements well below those feasible with conventional counter systems and has application not only to current biophysical problems, but also to astrophysical and nuclear structure investigations.

To extend studies in range, etch rate, and etch induction time for heavy ions in cellulose nitrate detectors, the University of Washington. Nuclear Physics Laboratory ${ }^{6} \mathrm{Li}$ beam and $90^{\circ}$ Brown-Buechner magnetic spectrometer were employed to obtain $6_{\mathrm{Li}}$ calibration points. These points have shown that the useful parameter of "etch induction time" is indeed unique for each species of incident ion and thus indicates that it will eventually be generally useful in a great variety of problems requiring heavy-ion spectroscopy for specific energy below $1 \mathrm{MeV} / \mathrm{amu}$. $\dagger$ Department of Physics, Washington State University, Pullman, WA. 


\subsection{Nuclear Physics Laboratory Personnel}

\section{Faculty}

Eric G. Adelberger, Professor

John S. Blair, Professor

David Bodansky, Professor

David F. Burch, Research Assistant Professor

John G. Cramer, Professor

Klaus A. Eberhard, Visiting Associate Professor ${ }^{1}$

George W. Farwell, Professor; Vice President for Research ${ }^{2}$

I. Halpern, Professor

Fred H. Schmidt, Professor

Kurt A. Snover, Research Assistant Professor

Robert Vandenbosch, Professor

William G. Weitkamp, Research Associate Professor;

Technical Director, Nuclear Physics Laboratory

Research Staff

Robert Bangert, Research Associate ${ }^{3}$

Hans-Holger Bohn, Research Associate

Philip A. Dickey, Research Associate

Peggy L. Dyer, Research Associate

Claus-Konrad Gelbke, Senior Research Associate

Lynn D. Knutson, Research Associate

Rainer Sielemann, Research Associate

Thomas A. Trainor, Research Associate

Laboratory Supervisory Personnel

Harold Fauska, Research Electronics Supervisor; Assistant

Technical Director, Nuclear Physics Laboratory

John W. Orth, Accelerator Engineer; Assistant Technical

Technical Director, Nuclear Physics Labor'atury

Predoctoral Research Associates

Chemistry

Man-Yee B. Tsang

Michael P. Webb

Physiçs

Norman L. Back

Klaus G. Bernhardt

Hyoung $C$. Bhang

John E. Bussoletti

Yuen-dat Chan
David T.C. Chiang

Bernardo D. Cuengco

Katsuyaki Ebisawa

Kwok-Leung Liu

William G. Lynch 
Research Assistants

Physics

Kelly C. Green

James D. Killian 7

James C. Wiborg

Full-Time Technical Staff

Professional Staff

Noel R. Cheney, Computer Systems Engineer

William B. Ingalls, Research Scientist

Shirley Kellenbarger, Chemist, Detector Maker

Gary W. Roth, Physicist

Rod E. Stowell, Electronics Engineer

H. Exik Swancon, Reocarch Engineer

Accelerator Technicians

Barbara L. Lewellen

Carl E. Linder

Georgia J. Rohrbaugh

George E. Saling

Design and Drafting

Peggy Douglass, Graphics Des./Illustrator Lewis E. Page, Designer

Instrument Makers

Louis L. Geissel

Nonman E. Gilbonteon

Charles E. Hart, Foreman

Gustàv t'. Johnson

Byron A. Scott, Student Shop Leadman

Allen L. Willman, Leadman

Administrative Staff

Susan Lambert, Secretary

Helene Turner, Administrative Secretary 
John Amsbaugh

Michael Anderson 8

James Burger 8

David Chamberlin

Solomon Davis ${ }^{8}$

Ronald Dickens

Lila Graham

Lynn Green

John Hastings ${ }^{8}$

Richard Holladay

Mark Killinger

Rochelle Kochin

David Palmer 8

Douglas Potter 8
Mojtaba H. Rezvani ${ }^{8}$

Tom Rigert ${ }^{8}$

Jan Sanislo

Edwin Selker 8

Kim Skoog 8

William Sprague ${ }^{8}$

Steven. Stradley

Charles Sum 8

Robert Thoreson

Pedro Valverde 8

Kody Van Dyk

Gary Walters ${ }^{8}$

Richard Weisfeld

Mark York 8

1. Present address: Universität München, Sektion Physik, D-8046 Garching, Germany.

2. On leave from the Department of Physics.

3. Present address: Universität zu Köln, Institut für Kernphysik, Kठln, Germany.

4. Permanent address: Institut für Kernphysik und Nukleare Festkörperphysik, Technische Univ. München, D-8046 Garching, Germany.

5. Permanent address: Max-Planck-Institut fü Kernphysik, 69 Heidelberg, Germany; now at Lawrence Berkeley Laboratory, University of California, Berkeley, CA 94720.

6. Permanent address: Hahn-Meitner-Institut für Kernforschung Berlin, 1 Berlin 39, Germany.

7. Terminated in December 1975.

8. Terminated.

18.2 Advanced Degrees Granted, Academic Year 1975-1976

H. Erik Swanson: Ph.D. "A Measurement of the Parity Violating Asymmetry in the Angular Distribution of $110 \mathrm{keV}$ De-excitation Radiation from Polarized 19F\% Nuclei".

Dennis Lowell oberg: Ph.D. "Production of ${ }^{6} \mathrm{Li},{ }^{9} \mathrm{Be}$ and ${ }^{10} \mathrm{~B}_{\mathrm{B}}$ in $\mathrm{p}+{ }^{13} \mathrm{C}$ Reactions from Threshold to $18 \mathrm{MeV}$ ". (Granted 1974, but omitted in previous listings.) 
Published Papers:

"A New Technique for the Bunching of Charged Particle Beams", John G. Cramer, Nucl. Instrum. Methods 128, 597 (1975).

"Suggestions for a Charge-State 'Enforcer' for Heavy Ion Accelerators", John G. Cramer, Nucl. Instrum. Methods 130, 121 (1975).

"On the production of Lithium,.Beryllium and Boron at Low Energies", D. Bodansky, W.W. Jacobs, and D.L. Oberg, Astrophys. J. 202, 222 (1975).

"Fore-Aft Anisotropy in the Radiative Capture of $14 \mathrm{MeV}$ Neutrons", E.D. Arthur, D.M. Drake and I. Halpern, Phys. Rev. Lett. 35, 914 (1975).

"Mimor Gàmma Decays in ${ }^{13} \mathrm{C}$ and ${ }^{13} \mathrm{~N} "$, R.E. Marrs, E.G. Adelberger, K.A. Snover, and M.D. Cooper, Phys. Rev. Lett. 35, 202 (1975).

"Upper Limits for Isospin-Forbidden $\gamma$-Decays of $\mathrm{T}=2$ States in ${ }^{20} \mathrm{Ne}$ ", R.E. Marrs, E.G. Adelberger, K.A. Snover and M.D. Cooper, Nucl. Phys. A256, 1. (1976).

"Quadrupole Spin Flip in Elastic Scattering of Nucleons", J.S. Blair, M.P. Baker, and H.S. Sherif, Phys. Lett. 60B, 25 (1975).

"'Resonances' in ${ }^{12} \mathrm{C}\left({ }^{12} \mathrm{C}, \alpha\right){ }^{20} \mathrm{Ne}$ and ${ }^{12} \mathrm{C}\left({ }^{12} \mathrm{C},{ }^{8} \mathrm{Be}\right){ }^{16} \mathrm{O}$ Reactions at About $37 \mathrm{MeV}$ ", K.A. Eberhard and K.G. Bernhardt, Phys. Rev. C 13, 440 (1976).

"K-Shell Ionization of Carbon by 1 to 18-MeV Protons", D. Burch, Phys . Rev. A 12,2225 (1975).*

"Charge State Distributions for 0.1 to $1.0 \mathrm{MeV} \mathrm{Ne}^{+}+\mathrm{Ne}$ Collisions Using $\mathrm{K}$ X-ray Measurements", D.L. Matthews, R.J. Fortner, D. Burch, B. Johnson and C.F. Moore, Phys. Lett. 50A, 441 (1975).

"Recoil Effects on the Impact Parameter Dependence of X-ray Production in Heavy Ion Collisions", D. Burch and K. Taulbjerg, Phys. Rev. A 12 , 508 (1975).

"Auger-electron and X-ray Production in 50- to 2000-keV Ne + Ne Collisions", N. Stolterfoht, D. Schneider, D. Burch, B. Aagaard, E. Boving, and B. Fastrup, Phys. Rev. A 12, 1313 (1975).

"Heavy-Ion Excitation of Autoionization States in Helium", D. Burch, J. Bolger, D. Schneider, and C.F. Moore, Phys. Rev. Lett. 36, 166 (1976).

"On the Question of Inner-Shell Electron Pickup during Alpha Decay", P. Dyer, D. Burch, H.J. Fischbeck, and M.S. Freedman, Phys. Rev. Lett. 36, 903 (1976): 
"Search for Coherent Excitation of X-ray Transitions in Channelled Ions", D. Burch and A.D. Marwick, Harwell Technical Report, AERE-M2742 (Sept. 1975).

"One-Neutron and Two-Neutron Transfer in the Scattering of ${ }^{18} 0$ by ${ }^{16}{ }_{0 "}$, W.N. Reisdorf, P.H. Lau, and R. Vandenbosch, Nucl. Phys. A235, 490 (1975).

"Energy Dependence of Deeply Inelastic Scattering of ${ }^{84} \mathrm{Kr}$ from ${ }^{208} \mathrm{~Pb}$ ", R. Vandenbosch, M.P. Webb, and T.D. Thomas, Phys. Rev. Lett. 36, 459 (1976). $" 180+{ }^{12} \mathrm{C}$ System: Evidence for Nonstatistical Intermediate Structure with Enhancement of Odd Partial Waves in the Reaction Channel", M.P. Webb, R. Vandenbosch, K.A. Eberhard, K.G. Bernhardt, and M.S. Zisman, Phys. Rev. Lett. 36, 779 (1976).

"The $\left({ }^{12} \mathrm{C},{ }^{8} \mathrm{Be}\right)$ Reaction on ${ }^{12} \mathrm{C},{ }^{16} \mathrm{O},{ }^{24,}{ }^{26} \mathrm{Mg},{ }^{40,48} \mathrm{Ca},{ }^{56} \mathrm{Fe}$ and ${ }^{58} \mathrm{Ni}$ between 50 and $65 \mathrm{MeV}$ Bombarding Energy", E. Mathiak, K.A. Eberhard, J.G. Cramer, H.H. Rossner, J. Stettmeier, and A. Weidinger, Nucl. Phys. A259, 129 (1976).

Papers Submitted or in Press:

"Isospin Mixing in ${ }^{12}$ C", E.G. Adelberger, R.E. Marrs, K.A. Snover, and J.E. Bussoletti, Phys. Lett., to be published.

"Calculations and Measurements of Radiative Proton Capture into ${ }^{15} \mathrm{~N}$ ", K.A. Snover, J.E. Bussoletti, K. Ebisawa, T.A. Trainor and A.B. McDonald, submitted to Phys. Rev. Lett.

"Evidence for Shallow Heavy Ion Optical Potentials", J.G. Cramer, R.M. DeVries, D.A. Goldberg, M.S. Zisman, and C.F. Maguire, submitted to Phys. Lett.

"Effects of Non-local Potentials in Heavy Ion Reactions", J.G. Cramer and R.M. DeVries, Phys. Rev. C, to be published.

"Deeply Inelastic Scattering of ${ }^{84} \mathrm{Kr}$ from ${ }^{208} \mathrm{~Pb}$, R. Vandenbosch, M.P. Webb, and T.D. Thomas, Phys. Rev. C, to be published.

"Elastic Scattering of ${ }^{84} \mathrm{Kr}$ from ${ }^{208} \mathrm{~Pb}$, R. Vandenbosch, M.P. Webb, T.D. Thomas, S.W. Yates, and A.M. Friedman, Phys. Rev. C; to be published.

"Application of Austern-Blair Theory to the Interference between Coulomb and Nuclear Excitation in the Inelastic Scattering of Heavy Ions", C.K. Gelbke and J.G. Cramer, submitted to Phys. Rev. C.

"High-Energy Levels in ${ }^{13} \mathrm{~N}: I I "$, D. Berghofer, M.D. Hasinoff, R. Helmer, B.T. Lim, and K. Ebisawa, submitted to Nucl. Phys.

"Nuclear Reactions in the ${ }^{86} \mathrm{Kr}+{ }^{139} \mathrm{La}$ System at $710 \mathrm{MeV}$, M.P. Webb, R. Vandenbosch, and T.D. Thomas, submitted to Phys. Lett. 
"Energy, Angular and Charge Distributions for Deeply Inelastic Scattering of Xe by $\mathrm{Ta}$ and $\mathrm{Pb} ", \mathrm{R}$. Vandenbosch, M.P. Webb, T.D. Thomas and M.S: Zisman, submitted to Nucl. Phys.

"Scattering of Polarized Protons from ${ }^{12} \mathrm{C}$ from 11.5 to $18 \mathrm{MeV}$ ", H.O. Meyer, W.G. Weitkamp, J.S. Dunham, T.A. Trainor and M.P. Baker, submitted to Nucl. Phỳs.

"The Energy Controversy: The-Fight over Nuclear Power", Fred H. Schmidt and David Bodansky, Albion Publishing Co., to be published.

"Neutron Pickup by Alpha Particles to Unbound States:, D.R. Brown, I. Halpern, J.R. Calarco, P.A. Russo, D.L. Hendrie and H. Homeyer, submitted to Phys. Rev. C.

"Isospin Forbidden Particle Decays in Light Nuclei (I): $T=3 / 2$ Levels in $9_{\text {Be, }} 9_{B "}$, A.B. McDonald, T.K. Alexander, O. Häusser, D. Disdier, E.G. Adelberger, H.B. Mak, A.P. Shukla and A.V. Nero, submitted to Nucl. Phys.

\section{Invited Papers:}

"Radiative Proton Capture Measurements and Model Calculations:, K.A. Snover, Invited paper at the APS Austin Meeting, Bull. Am. Phys. Soc. 20, 1186 (1975).

"Nuclear Parity Mixing", E.G. Adelberger, Invited talk given at 1975 Gordon Research Conference on Nuclear Chemistry.

"Heavy-ion Charge States and Multiple Scattering", D. Burch, Invited paper presented at LBL SuperHILAC Users Meeting, Berkeley, Dec. 16, 1975.

"D-State Effects in Transfer Reactions", L.D. Knutson, Invited päper, presented at the Fourth International Symposium on Polarization

Phenomena in Nuclear Reactions, Zïich, 1975, to be published.

"Parity Mixing", E.G. Adelberger, Invited paper presented at Fourth International Symposium on Polarization Phenomena in Nuclear Reactions, Zurich, 1975 , to be published.

\section{Conference Proceedings Publications:}

"Depolarization in the Elastic Scattering of 17-MeV Protons from 9 Be", M.P. Baker, J.S. Blair, J.G. Cramer, T. Trainor, and W. Weitkamp, in "Contributed Papers to the Fourth International Symposium on Polarization Phenomena in Nuclear Reactions", Zurich, 1975, to be published.

"Quadrupole Contribution to the Depolarization of Nucleons Scattered by Deformed Nuclei", J.S. Blair, M.P. Baker, and H.S. Sherif, in "Contributed Papers to the Fourth International Symposium on Polarization Phenomena in Nuclear Reactions", Zurich, 1975, to be published. 
"Analyzing Power in the ${ }^{206} \mathrm{~Pb}(\mathrm{p}, \mathrm{pO}){ }^{206} \mathrm{~Pb}$ Reaction Near the ${ }^{3} \mathrm{P}_{1 / 2}$ Isobaric Analog Resonance", M.P. Baker, T.A. Trainor, J.S. Blair, J.G. Cramer, and W.G. Weitkamp, in "Contributed Papers to the Fourth International Symposium on Polarization Phenomena in Nuclear Reactions", Zurich, 1975, to be published.

"Analyzing Power for Proton-Elastic Scattering from ${ }^{208} \mathrm{~Pb}$ Near the Low-Lying Isobaric Analog Resonances", M.P. Baker, J.S. Blair, J.G. Cramer, E. Preikschat and W. Weitkamp, in "Contributed Papers to the Fourth International Symposium on Polarization Phenomena in Nuclear Reactions", Zurich, 1975, to be published.

"Scattering of Polarized Protons from Carbon from 11.5 to $18 \mathrm{MeV}$ ". W.G. Weitkamp, H.O. Meyer, J.S. Dunham, T.A. Trainor and M.P. Baker, in "Contributed Papers to the Fourth International Symposium on Polarization Phenomena in Nuclear Reactions", Zlirich, 1975, to be published.

"Analyzing Power for Elastic Scattering of Protons from 13C", T.A. Trainor, N.L. Back, J.E. Bussoletti, and L.D. Knutson, in "Contributed Papers to the Fourth International Symposium on Polarization Phenomena in Nuclear Reactions", Zurich, 1975, to be published.

"The University of Washington Polarized Ion Source", T.A. Trainor and W.B. Ingalls, in "Contributed Papers to the Fourth International Symposium on Polarization Phenomena in Nuclear Reactions", Zurich, 1975, to be published.

"The Spin Rotation Angle in ${ }^{12} c(p, p){ }^{12} c$ Scattering", W.G. Weitkamp, W. Grliebler, V. Kornig, P.A. Schmelzbach, R. Risler and B. Jenny, in "Contributed Papers to the Fourth International Symposium on Polarization Phenomena in Nuclear Reactions", Zurich, 1975, to be published.

"Polarization Transfer Coefficients for ${ }^{3} \mathrm{He}(\vec{p}, \vec{p}){ }^{3}$ He Scattering below 11 MeV", W.G. Weitkamp, W. Gruebler, V. König, P.A. Schmelzbach, R. Risler and B. Jenny, in "Contributed Papers to the Fourth International Symposium on Polarization Phenomena in Nuclear Reactions", Zurich, 1975, to be published.

"Measurement of the Analyzing Powers in ${ }^{3} \mathrm{He}(\mathrm{d}, \mathrm{d})^{3} \mathrm{He}$ Scattering", B. Jenny, W. Griebler, V. K४nig, P.A. Schmelzbach, R. Risler, D.0. Boerma and W.G. Weitkamp, in "Contributed Papers to the Fourth International Symposium on Polarization Phenomena in Nuclear Reactions", Zurich, 1975, to be published.

"Analyzing Power Maxima $A_{y}=-1$ and $A_{y y}=1$ in $d-\alpha$ Elastic Scattering", W. Grllebler, P.A. Schmelzbach, V. König, R. Risler, B. Jenny D.O. Boerma and W.G. Weitkamp, in "Contributed Papers to the Fourth International Symposium on Polarization Phenomena in Nuclear Reactions", Zurich, 1975, to be published.

"Deeply Inelastic Scattering of Xe by . Ta and $\mathrm{Pb}$ ", M.S. Zisman, R. Vandenbosch, M.P. Webb and T.D. Thomas, Proceedings of the Macroscopic Features of Heavy Ion Collisions, ANL, 1-3 April, 1976, to be published. 
"Measurement of the Tensor Analyzing Power Ayy near the Value $A_{y}=1$ in $d-\alpha$ Elastic Scattering", G. Heidenreich, V. König, W. Grllebler, P.A. Schmelzbach, R. Risler, B. Jenny, and W.G. Weitkamp, in "Contributed Papers to the Fourth International Symposium on Polarization Phenomena in Nuclear Reactions", Zurich; 1975, to be published.

$" \vec{p}+{ }^{12} \mathrm{c}$ streuung zwischen $12 \mathrm{MeV}$ und $18 \mathrm{MeV}$ ", H.O. Meyer, W.G. Weitkamp and J.S. Dunham, Helv. Phys. Acta 48, 559 (1975).

"Excitation of Autoionization States in He by $30-\mathrm{MeV}$ Oxygen Ions", D. Burch, J. Bolger and C.F. Moore, Proc. of IXth International Conf. on Phys. of Electronic and Atomic Collisions, Seattle, 1975, J.S. Risley and R. Geballe, eds., p. 863 .

"Continuous Photon Spectrum of $60-\mathrm{MeV} \mathrm{Cl}+\mathrm{Pb}$ Collisions at Zero Impact Parameter", D. Burch, W. Ingalls, H. Wieman, and R. Vandenbosch, Proc. of IXth International Conf. on Phys. of Electronic and Atomic Collisions, Seattle, 1975, J.S. Risley and R. Geballc, edo., p. 306.

Contributed Abstracts:

"Particle Decays of $\mathrm{T}=2$ States in Mass 8", E.G. Adelberger, J.S. Freedman, A.V. Nero, A.B. McDonald, R.G.H. Robertson and D.R. Goosman, Bull. Am. Phys. Soc. 20, 596 (1975).

"Analysis of the ${ }^{89} \mathrm{Y}\left(\mathrm{p}, \gamma_{0}\right)$ Reaction with the Direct-Semidirect Model", F.S. Dietrich, K.A. Snover, and K. Ebisawa, Bull. Am. Phys. Soc. 20, 1160 (1975).

"Nuclear Reactions Induced by the Bombardment of ${ }^{139} \mathrm{La}$ with ${ }^{84} \mathrm{Kr}$ at 700 MeV", M.P. Webb, R. Vandenbosch, and T.D. Thomas, Bull. Am. Phys. Soc. 20, $1175(1975)$.

"Tests of the Sensitivity of the Heavy Ion Optical Model to Details of the Nuclear Potential", J.G. Cramer, Bull. Am. Phys. Soc. 20, 1159 (1975).

"Evidence for Shallow, Very Strongly Absorbing Heavy-Ion Uptical

Potentials", J.G. Cramer, R.M. DeVries, D.A. Goldberg, M.S. Zisman, and C.F. Maguire, Bull. Am. Phys. Soc. 21, 64 (1976).

"Resonanzerscheinungen in Leichten Schwerionen-Systemen", K.A. Everhard, K.G. Bernhardt, H. Bohn, R. Vandenbosch, M.P. Webt, and M.S. Zisman, DPG Meeting, March 29-April 2, 1976, Baden/Wien, Austria.

$"\left({ }^{6} \mathrm{~L},{ }^{6} \mathrm{LI}\right)$ und $\left({ }^{6} \mathrm{Li}, \alpha\right)$ an ${ }^{40} \mathrm{Ca}$ und ${ }^{44} \mathrm{Ca} ", \mathrm{H}$. Bohn, K.A. Ebcrhard, K.G. Bernhardt, R. Bangert, Y.D. Chan, and R. Vandenbosch, DPG Meeting, March 29-April 2, 1976, Baden-Wien, Austria.

"6i: A Light Heavy Ion or a Heavy Light Ion?" J.G. Cramer, R.M.' DeVries, D.A. Goldberg, M.S. Zisman, and C.F. Maguire, Bull. Am. Phys. Soc., 21, 554 (1976).

"Measurements of $\pi^{ \pm}$Nucleus Total X-sections at Energies below $200 \mathrm{MeV}$ ", G. Burleson, M. Jakobson, I. Halpern, M. Cooper et al., Paper 1.D.6, Sixth Int. Conf. of High Energy Physics and Nuclear Structure, Santa Fe, NM, 1975. 


\section{Other Publications by Members of the Laboratory:}

"Safety Aspects of Nuclear Energy", D. Bodansky and F.H. Schmidt, in "The Nuclear Power Controversy", Arthur W. Murphy, ed., American Assembly, Columbia University (1976); to be published.

"Deuteron D-state Effects for the Reactions ${ }^{117} \mathrm{Sn}(\overrightarrow{\mathrm{d}, \mathrm{p}})^{118} \mathrm{Sn}$ and $119 \mathrm{Sn}(\mathrm{d}, \mathrm{p}) 120 \mathrm{sn}^{\prime}$, L.D. Knutson, J.A. Thomson and H.O. Meyer, Nucl. Phys. A241, 36 (1975).

"Polarization in Proton-Proton Scattering at $10 \mathrm{MeV}$ ", J.D. Hutton, W. Haeberli, L.D. Knutson, and P. Signell, Phys. Rev. Lett. 35, 429 (1975).

"Probing the Deuteron Wave Function with sub-Coulomb ( $d, p)$ Reactions", L.D. Knutson and W. Haeberli, Phys. Rev. Lètt. 35, 558 (1975).

"238 $\mathrm{U}$ and ${ }^{232}$ Th Photofission and Photoneutron Emission Near Threshold", P.A. Dickey and P. Axel, Phys. Rev. Lett. 35, 501 (1975).

"Wave Functions of Nuclear States with Small Single-Particle Spectroscopic Factors", K.G. Bernhardt, W.R. Hering, P. Ring, and E. Werner, Nucl. Phys. A249, 329 (1975).

"Spin-Dependent Potentials for Deuteron-Nucleus Scattering", L.D. Knutson and W. Haeberli, Phys. Rev. C 12, 1469 (1975).

"Effects of the Triton D-state in $(d, t)$ Reactions", L.D. Knutson, B.P. Hichwa, A. Barroso, A.M. Eir6, F.D. Santos, and R.C. Johnson, Phys. Rev. Lett. 35, 1570 (1975).

"The $j$-dependence of the Vector Analyzing Power for $(d, n)$ Reactions on $58 \mathrm{Ni}$ and $60 \mathrm{Ni} "$, B.P. Hichwa, L.D. Knutson, J.A. Thomson, W.H. Wong, and P.A. Quin, to be published (Nucl. Phys.).

"K-Matrix Parameterization of the ${ }^{12} \mathrm{C}(\alpha, \gamma){ }^{16} 0$ Cross Section", J. Humblet, P. Dyer and B.A. Zimmerman, submitted to Nucl. Phys.

"Search for Isovector Giant E2 States in ${ }^{12} \mathrm{C}$ and ${ }^{16} \mathrm{O}$, P. Paul, J.W. Noe, K.A. Snover, and M. Suffert, Intemational Symposium on Highly Excited States in Nuclei, Julich, W. Germany, 1975, Vol. 1, p. 2 .

"Structure of Excited States in ${ }^{100}$ Mo and ${ }^{102}$ Mo from Spectroscopy Using 180 Induced Reactions", H. Bohn, P. Kienle, D. Proetel, and R.L. Hershberger, Z. Physik A274, 327 (1975).

${ }^{12} \mathrm{C}\left({ }^{12} \mathrm{C},{ }^{8} \mathrm{Be}\right){ }^{16} \mathrm{O}$ Angular Distributions and Excitation Functions between 35 and $69 \mathrm{MeV}$ Bombarding Energy", A. Weidinger, K.A. Eberhard, E. Mathiak, J. Stettmeier, W. Trombik, and L.N. Wustefeld, Nucl. Phys. A257, 144 (1976). 
"Studium von neutronenarmen Se, $\mathrm{Kr}$, Sr Kernen mit Hilfe von Schwerioneninduzierten Compoundreaktionen:, H. Bohn, T. Faestermann, F.v. Feilitzsch, P. Kienle, G. Korschinek, M.R. Maier, and E. Nolte, Verhandl DPG (VI) 10, 739 (1975).

"Half Lives of Excited States in ${ }^{100}$ M and ${ }^{102}$ Mo", H. Bohn, P. Kienle, D. Proetel, and R.I. Hershberger, Verhandl DPG (VI) 10, 775 (1975).

"Inelastische Streuung von Sauerstoff-und Schwefel Ionen", H. Bohn, H. Essel, H.J. Körner, K.E. Rehm, H. Rödig, H. Spieler, and J.P. Schiffer, Verhandl DPG (VI) 10, 735 (1975).

"Suchenach Angeregten Zustanden in ${ }^{206} \mathrm{Hg}$ mit der Reaktion ${ }^{204} \mathrm{Hg}\left(18_{0}, 16_{0} \gamma\right)^{206} \mathrm{Hg}^{\prime \prime}, \mathrm{W}$. Hering, H. Puchta, W. Trautmann, and H. Bohn, Verhandl DPG (VI) 10, 857 (1975).

"Electric Quadrupole Interaction of Recoil-Implanted Zinc in Cadmium", W. Bartsch, J. Golczewski, W. Leitz, H.-E. Mahnke, W. Semmler, R. Sieleilaun, and Th. Wickert, Phys. Lett. 50A, 413 (1975).

"Time-Differential Observation of Quadrupole Interactions Following Recoil Implantation of a Ge-Probe into $\mathrm{Cd}$, In, $\mathrm{Sn}$, and $\mathrm{Sb}^{\prime \prime}$, W. Bartsch, W. Leitz, H.-E. Mahnke, W. Semmler, R. Sielemann, and Th. Wickert, Z. Physik B21, 131, (1975).

"Probe Specific Temperature Dependence of the Quadrupole Interaction of 67 Ge in Zinc Metal", W. Bartsch, W. Leitz, W. Semmler, R. Sielemann, and Th. Wickert, Phys. Lett. 54A, 66 (1975).

"The Half-Lives of the First and Second Excited State in "66", W. Leitz, H.-E. Mahnke, W. Semmler, R. Sielemann, and Th. Wickert, Nucl.Phys.A258, 103 (1976).

"Influence of the Impurity on the Solute Electric Field Gradient in NonCubic s-p Metals", W. Leitz, W. Semmler, R. Sielmann, and Th. Wickcrt, submitted to Phys. Rev. Lett.

"Electric-Field Gradients of Recoil-Implanted ${ }^{19} \mathrm{~F}$ in Metallic Backings via the Reaction ${ }^{\mathrm{Be}}\left({ }^{12} \mathrm{C}, \mathrm{np}\right)^{19} \mathrm{~F} ", \mathrm{C}$. Budtz-J $\not$ rgensen, K. Bonde Nielsen, F. Abildskov; T.K. Laursen, W. Semmler, R. Sielemann, and Th. Wickert, in International Meeting on Hypertine Interactions, Leuven, Belgium, 1975.

"Temperature Dependence of the Quadrupole Interdclion of the Impurity 67Ge in $\mathrm{Zn}, \mathrm{Cd}$, and Sn", W. Bartsch, B. Lamp, W. Lcitz, H. -E. Mahnke, W. Semmler, R. Sielemann, and Th. Wickert, in International Meeting on Hyperfine Interactions, Leuven, Belgium, 1975. 


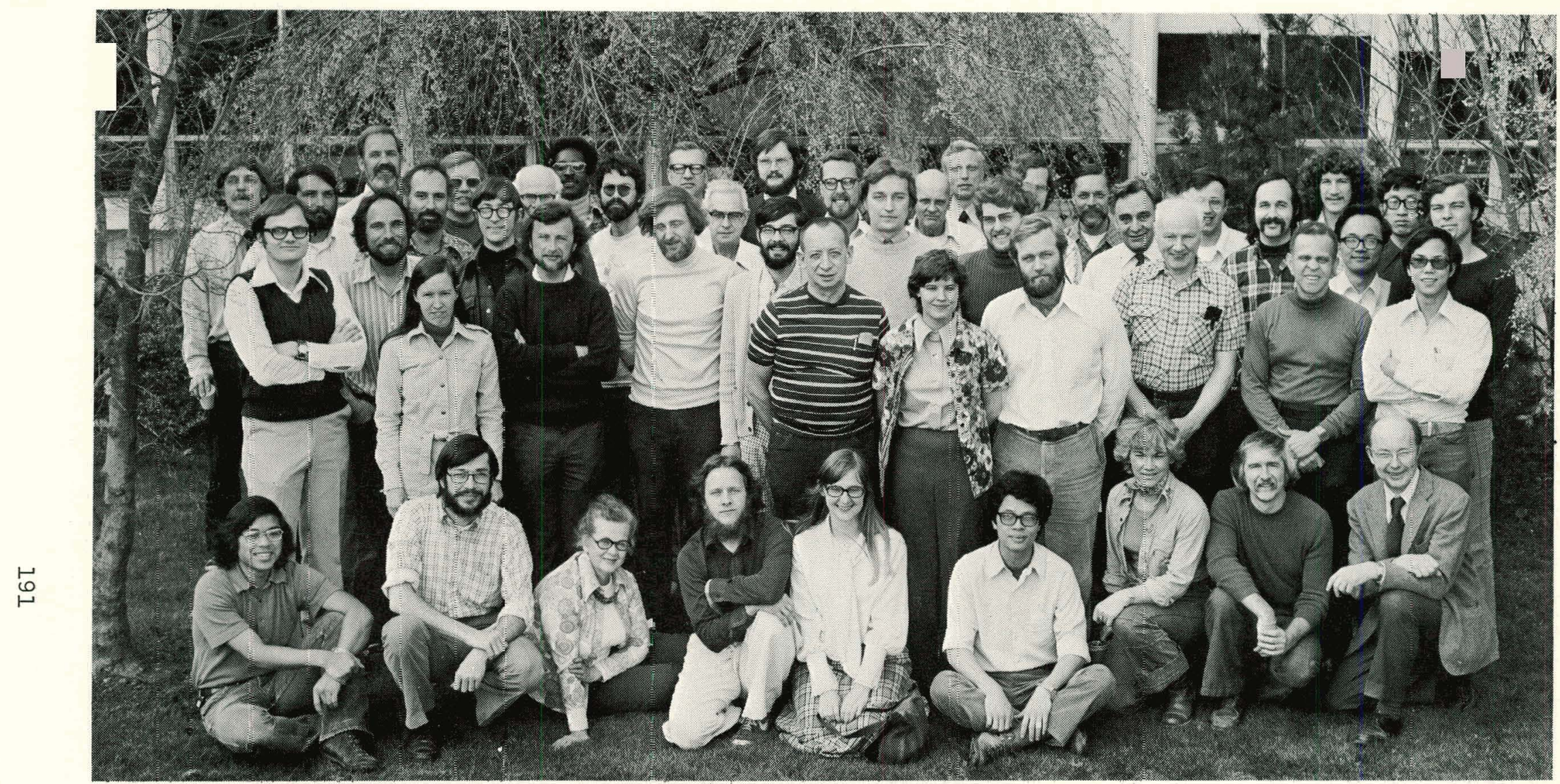

Hinn Weitkamp Linder Dickens Cheney MacLellan Vandenbosch

Wiborg Calarco Schmidt

Adelberger Cramer Ingalls Scott

Webb

Swansc

Cuengøo

Trainor

Douglass

Holladay

Green

Back Gilbertson Roth Willman Bussoletti Lynch Bhang

Bohn Bernhardt Bodansky Dickey Ebisawa Chamberlin

ka Lewellen Burch Orth Page Liu 
